\title{
A BDI empathic agent model based on a multidimensional cross-cultural emotion representation
}

\author{
JOAQUIN TAVERNER APARICIO
}

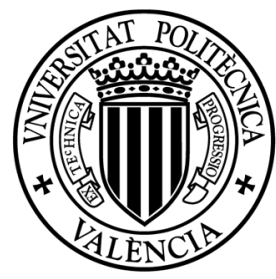

UNIVERSITAT

POLITÈCNICA

DE VALÈNCIA

Valencian Research Institute For ARTificial

INTELLIGENCE (VRAIN)

A thesis submitted for the degree of

Doctor of Philosophy in Computer

Science

Supervised by

Dr. Vicente Botti

Dr. Emilio Vivancos

January 2022 

Supervisors

Dr. Vicente Botti

Valencian Research Institute for Artificial Intelligence (VRAIN), Universitat Politècnica de València (Spain)

Dr. Emilio Vicancos

Valencian Research Institute for Artificial Intelligence (VRAIN), Universitat Politècnica de València (Spain)

External Reviewers

Dr. Abe Kazemzadeh

University of St. Thomas, United States

Dr. Juan Manuel Corchado

University of Salamanca, Spain

Dr. Stacy Marsella

Khoury College of Computer Sciences, United States
Thesis Defense Committee Members

Dr. Ana Paiva

University of Lisbon, Portugal

Dr. Juan Manuel Corchado

University of Salamanca, Spain

Dr. Vicente Julian Inglada

Universitat Politècnica de València, Spain

Ph.D. Thesis

(C)Joaquin Taverner Aparicio. Valencia, Spain 2022.

This work is subjected to copyright. All rights are reserved.

This thesis has been partially supported by the Generalitat Valenciana and European Social Fund by the FPI grant ACIF/2017/085 and by the Spanish Government project PID2020113416RB-I00. 
-

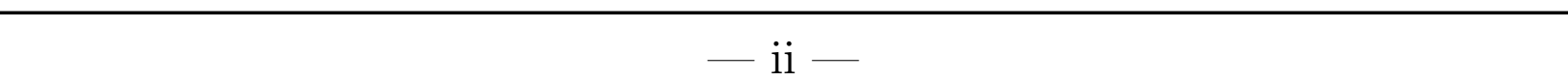




\section{Abstract}

Human beings are, by nature, affective beings; our emotions, moods, personality, or relationships with others guide our motivations and our decisions. One of the main cognitive abilities related to affect is empathy. Empathy is a psychological construct whose definition has evolved over the years and whose meaning refers to a wide range of affective and cognitive competencies that are fundamental in the development of human beings as social beings. The use of empathy in the field of artificial intelligence can revolutionize the way we interact with machines as well as the way we simulate human behavior.

On the other hand, it must be considered that human beings usually resort to the use of different words such as "sad" or "happy" to express or verbalize our affective state. However, these words are simplifications that cover a wide spectrum of cognitive processes and mental states. Moreover, it should be considered that these words have a high dependence on the language and culture in which they are used. Therefore, computational representation models of affective states must adaptable to different cultural environments and to allow an agent to express or represent, by means of words, a given affective state.

In this thesis, a new model of empathic agent capable of adapting its behavior to different cultural environments is proposed. To this end, first, a new experiment-based methodology to adapt an emotion representation space based on the dimensions of pleasure and arousal for simulation and affective computational recognition to different cultural environments is presented. The results of an experiment conducted with European Spanish speakers are used to provide a new fuzzy logic-based model for representing affective states in the dimensions of pleasure and arousal using a polar coordinate approach. To prove that cultural differences affect the pleasure and arousal values associated with each emotion, the experiment was replicated with Portuguese and Swedish participants. Secondly, a new model of emotion elicitation in affective agents using fuzzy logic is presented. The emotions generated in the agent by the fuzzy appraisal rules are expressed in the model of affect representation resulting from the previously described experiments. In addition, a new affect regulation process is proposed to adapt the agent's mood, 
represented by a vector in the pleasure-arousal space, when an emotion is elicited. Third, a formalization of the syntax, semantics and reasoning cycle of AgentSpeak to enable the development of affective agents with empathic capabilities is presented. Drawing on the theories of empathic appraisal and empathic regulation, the agent's reasoning structure is modified to allow empathy to affect the decision-making process. Finally, a model of an empathic pedagogical agent for education on good practices in the use of social networks is introduced. The agent is able to recognize the user's emotion when interacting with the social network. Based on the user's emotion and behavior in the social network, the agent estimates a plan to educate the user in the correct and secure use of social networks. 


\section{Resumen}

Los seres humanos somos por naturaleza seres afectivos, las emociones, el estado de ánimo, nuestra personalidad, o nuestras relaciones con los demás guían nuestras motivaciones y nuestras decisiones. Una de las principales habilidades cognitivas relacionadas con el afecto es la empatía. La empatía es un constructo psicológico cuya definición ha ido evolucionando a lo largo de los años y cuyo significado hace referencia a un amplio abanico de competencias afectivas y cognitivas que son fundamentales en el desarrollo del ser humano como ser social. El uso de la empatía en el ámbito de la inteligencia artificial puede revolucionar la forma en la que interactuamos con las máquinas así como la forma en la que simulamos el comportamiento humano.

Por otro lado, hay que tener en cuenta que los seres humanos habitualmente acudimos al uso de distintas palabras como "triste" o "contento" para expresar o verbalizar el estado afectivo. Sin embargo, estas palabras son simplificaciones que abarcan un amplio espectro de procesos cognitivos y estados mentales. Además, hay que considerar que estas palabras tienen una alta dependencia del idioma y la cultura en la que se utilizan. Por tanto, los modelos de representación computacional de los estados afectivos deben se capaces de adaptarse a distintos entornos culturales y de permitir que un agente exprese o represente, mediante palabras, un determinado estado afectivo.

En esta tesis se propone un nuevo modelo de agente empático capaz de adaptar su comportamiento a distintos entornos culturales. Para ello, en primer lugar, se presenta una nueva metodología basada en la experimentación para adaptar un espacio de representación de emociones basado en las dimensiones del placer y la activación para la simulación y el reconocimiento computacional afectivo a diferentes entornos culturales. Los resultados del experimento realizado con hispanohablantes europeos se utilizan para proporcionar un nuevo modelo basado en la lógica difusa para representar estados afectivos en las dimensiones de placer y activación utilizando un enfoque de coordenadas polares. Para demostrar que las diferencias culturales afectan a los valores de placer y activación asociados a cada emoción, el experimento se repitió con participantes portugueses y suecos. En segundo lugar, se presenta un nuevo modelo de elicitación de emociones en agentes afectivos que utiliza 
lógica difusa. Las emociones generadas en el agente por las reglas de valoración difusa se expresan en el modelo de representación del afecto resultante de los experimentos previamente descritos. Además, se propone un nuevo proceso de regulación del afecto que adapta el estado de ánimo del agente, representado mediante un vector en el espacio placer-activación, cada vez que una emoción es elicitada. En tercer lugar, se propone una formalización de la sintaxis, la semántica y el ciclo de razonamiento de AgentSpeak para permitir el desarrollo de agentes afectivos con capacidades empáticas. Partiendo de las teorías de valoración empática y regulación empática, se modifica la estructura de razonamiento del agente para permitir que la empatía afecte al proceso de toma de decisiones. Finalmente, se presenta un modelo de agente pedagógico empático para la educación sobre buenas prácticas en el uso de las redes sociales. El agente es capaz de reconocer la emoción del usuario cuando interactúa con la red social. En base a la emoción del usuario y su comportamiento en la red social, el agente estima un plan para educar al usuario en el uso correcto y seguro de las redes sociales. 


\section{Resum}

Els éssers humans som per naturalesa éssers afectius, les emocions, l'estat d'ànim, la nostra personalitat o les nostres relacions amb els altres guien les nostres motivacions i les nostres decisions. Una de les habilitats cognitives principals relacionades amb l'afecte és l'empatia. L'empatia és un constructe psicològic la definició del qual ha anat evolucionant al llarg dels anys i el significat del qual fa referència a un ampli ventall de competències afectives i cognitives que són fonamentals en el desenvolupament de l'ésser humà com a ésser social. L'ús de l'empatia en l'àmbit de la intel-ligència artificial pot revolucionar la forma en la qual interactuem amb les màquines així com la forma en què simulem el comportament humà.

D'altra banda, cal tenir en compte que els éssers humans habitualment acudim a l'ús de diferents paraules com "trist" o "content" per expressar o verbalitzar l'estat afectiu. Tot i això, aquestes paraules són simplificacions que abasten un ampli espectre de processos cognitius i estats mentals. A més, cal considerar que aquestes paraules tenen una alta dependència de l'idioma i la cultura en què s'utilitzen. Per tant, els models de representació computacional dels estats afectius han de ser capaços d'adaptar-se a diferents entorns culturals i de permetre que un agent expresse o represente, mitjançant paraules, un estat afectiu determinat.

En aquesta tesi es proposa un nou model d'agent empàtic capaç d'adaptar el seu comportament a diferents entorns culturals. Per això, en primer lloc, es presenta una metodologia nova basada en l'experimentació per adaptar un espai de representació d'emocions basat en les dimensions del plaer i l'activació per a la simulació i el reconeixement computacional afectiu a diferents entorns culturals. Els resultats de l'experiment realitzat amb hispanoparlants europeus es fan servir per proporcionar un nou model basat en la lògica difusa per representar estats afectius en les dimensions de plaer i activació mitjançant un enfocament de coordenades polars. Per demostrar que les diferències culturals afecten els valors de plaer i activació associats a cada emoció, l'experiment es va repetir amb participants portuguesos i suecs. En segon lloc, es presenta un nou model d'elicitació d'emocions en agents afectius que fa servir lògica difusa. Les emocions generades a l'agent per les regles de 
valoració difusa s'expressen en el model de representació de l'afecte resultant dels experiments descrits prèviament. A més, es proposa un nou procés de regulació de l'afecte que adapta l'estat d'ànim de l'agent, representat mitjançant un vector a l'espai plaer-activació, cada cop que una emoció és elicitada. En tercer lloc, es proposa una formalització de la sintaxi, semàntica i cicle de raonament d'AgentSpeak per permetre el desenvolupament d'agents afectius amb capacitats empàtiques. Partint de les teories de valoració empàtica i regulació empàtica, es modifica l'estructura de raonament de l'agent per permetre que l'empatia afecti el procés de presa de decisions. Finalment, es presenta un model d'agent pedagògic empàtic per a l'educació sobre bones pràctiques en l'ús de les xarxes socials. L'agent és capaç de reconèixer l'emoció de l'usuari quan interactua amb la xarxa social. En base a l'emoció de l'usuari i el seu comportament a la xarxa social, l'agent estima un pla per educar l'usuari en l'ús correcte i segur de les xarxes socials. 
Quisiera dar las gracias a mi familia por todo el cariño que me brinda, a mi pareja por soportarme durante todos estos años, tanto en los momentos buenos como en los no tan buenos, a mis amigos por animarme a seguir adelante, a los que caminaron a mi lado y ya no están, a mis directores Vicent y Emilio que, además de ser unos excelentes mentores, siempre me han apoyado ofreciéndome su consejo y mostrando una paciencia infinita incluso en los momentos de mayor debilidad y flaqueza. También quisiera agradecerles a mis compañeros de laboratorio y a mis compañeros del VRAIN por su apoyo y su colaboración y en especial a Bexy con la que compartí mis inicios en esta aventura. 


\section{Contents}

I Introduction and objectives 1

$1 \quad$ Introduction and objectives 3

1.1 Motivation . . . . . . . . . . . . . . . . . 8

1.2 Objectives . . . . . . . . . . . . . . . . . 10

1.3 Structure of the thesis . . . . . . . . . . . . . . . . . . . 12

1.4 List of publications . . . . . . . . . . . . . . . . 14

1.5 List of projects . . . . . . . . . . . . . 16

$\begin{array}{ll}\text { II Contribution } & 17\end{array}$

2 A culturally adapted representation of emotions (Selected paper) 19

2.1 Introduction . . . . . . . . . . . . . . . 20

2.2 Related works . . . . . . . . . . . . . . . . . . . . 21

2.3 An experiment-based method to adapt affective models to culture and language . . . . . . . . . . . . . . . . . . 28 28

2.4 A fuzzy culturally adapted model of affect . . . . . . . . . 39

2.5 Conclusion and future work . . . . . . . . . . . . . 46

3 Computational affective knowledge representation 49

3.1 Introduction . . . . . . . . . . . . . . . 50

3.2 Related work . . . . . . . . . . . . . . . . . 51

3.3 A cross-cultural model of emotions for affective computing . . 56

3.4 Experiment design . . . . . . . . . . . . . . . . 60

3.5 Results . . . . . . . . . . . . . . . . . . . . . . . . 65 
3.6 Discussion . . . . . . . . . . . . . . . . . . . . . . . . 76

3.7 Applications . . . . . . . . . . . . . . . . 86

3.8 Conclusions . . . . . . . . . . . . . . . . . . . . . . 89

4 A fuzzy appraisal model for affective agents (Selected paper) 93 4.1 Introduction . . . . . . . . . . . . . . . . . . . 94

4.2 Previous work . . . . . . . . . . . . . . . . . . 95

4.3 A model for emotion elicitation in affective agents . . . . . . . 103

4.4 Conclusions and future work . . . . . . . . . . . . . . 117

$\begin{array}{lll}5 & \text { A formal extension for modelling empathic agents } & 121\end{array}$

5.1 Introduction . . . . . . . . . . . . . . . . . . . . 122

5.2 Related work . . . . . . . . . . . . . . . . . . . . 124

5.3 Formalization of the AgentSpeak extension to develop empathic agents . . . . . . . . . . . . . . . . 132

5.4 Conclusions . . . . . . . . . . . . . . . . . . 161

6 Towards a model of empathic pedagogical agent (Selected paper)

6.1 Introduction . . . . . . . . . . . . . . . . 166

6.2 Related work . . . . . . . . . . . . . . . . 167

6.3 Proposal . . . . . . . . . . . . . . . . . . . . . . . 172

6.4 Conclusions and future work . . . . . . . . . . . . . . 175

$\begin{array}{ll}\text { III Discussion } & 177\end{array}$

$\begin{array}{llr}7 & \text { Discussion } & 179\end{array}$

IV Conclusion and future work $\quad 185$

8 Conclusion and future work 187

$\begin{array}{ll}\text { Bibliography } & 191\end{array}$ 


\section{List of Figures}

2.1 The Circumplex Model of Affect . . . . . . . . . . . . . . . . 22

2.2 Wheel of emotions proposed by Plutchik $[172] \ldots \ldots$. . . . . 23

2.3 Degree of acceptance of the candidate words for each affective category. . . . . . . . . . . . . . . . . . 32

2.4 Circular boxplot graphic for the ten selected emotions in Spanish 34

2.5 Dispersion of the Disgust emotion. . . . . . . . . . . . . . 36

2.6 Von Mises curves of the ten Spanish emotions . . . . . . . . . 36

2.7 Results obtained in our experiment with emotions expressed in Spanish . . . . . . . . . . . . . . . 38

2.8 Levels of Pleasure and Arousal associated to Spanish emotions . . 41

2.9 Fuzzy model for the intensity. . . . . . . . . . . . . . . . . 41

2.10 Proposed fuzzy model for the type of the Spanish emotions . . . . 42

2.11 Example of a multicultural affective agent module. . . . . . . . . 46

3.1 Distribution of probability functions in the pleasure-arousal space using one standard deviation for Spanish. . . . . . . . . . . . 58

3.2 Degree of acceptance of the candidate words in Portuguese for each affective category. . . . . . . . . . . . . . . . 66

3.3 Degree of acceptance of the candidate words in Swedish for each affective category. . . . . . . . . . . . . . . . . . 69

3.4 Portugal experiment results. . . . . . . . . . . . . . . . . 73

3.5 Sweden experiment results. . . . . . . . . . . . . . . 75

3.6 Mixture of Von Mises curves of the ten emotions in Portuguese, Swedish, and Spanish. The axis represents the angles $\alpha_{e}$ in degrees. 77

3.7 Results obtained. . . . . . . . . . . . . . . . . . . . . . 78 
3.8 Distribution of probability functions in the pleasure-arousal space using one standard deviation for Portuguese. . . . . . . . . . . 81

3.9 Distribution of probability functions in the pleasure-arousal space using one standard deviation for Swedish. . . . . . . . . . . 82

3.10 A stress relief plan consists of a sequence of subgoals (G1-G3) and a final goal $(\mathrm{G} 4) . \ldots \ldots \ldots . \ldots . \ldots . \ldots 87$

4.1 The circumplex model of affect . . . . . . . . . . . . . . 96

4.2 The proposed fuzzy model for the intensity of the emotions in the Pleasure-Arousal space. . . . . . . . . . . . . . . . . . . . 110

4.3 Example of the estimation of the mood considering two different personalities. . . . . . . . . . . . . . . 116

5.1 Types of agents in a social interaction . . . . . . . . . . . 134

5.2 GenIA ${ }^{3}$ configuration. . . . . . . . . . . . . . . 135

5.3 New configuration of both affective and rational cycles. . . . . . . 139

5.4 Simplified extension of the agent's syntax including the new extension of GenIA $A^{3} \ldots \ldots \ldots$. . . . . . . . . . . . . 146

5.5 Simplified extension of the MAS project syntax including the new extension of GenIA ${ }^{3} \ldots \ldots \ldots$. . . . . . . . . . 146

6.1 The proposed model for a empathic pedagogical agent in a social network . . . . . . . . . . . . . . . . . 171

6.2 Screenshot of the Pesedia social network with the agent avatar interface on the right side. . . . . . . . . . . . . . . 175 


\section{List of Tables}

2.1 Results of the second experiment . . . . . . . . . . . . 35

2.2 Distribution of emotions in European Spanish and British English 39

2.3 Parameter ranges for the intensity membership function. . . . . . 43

2.4 Example of coefficients for estimating the intensity of an emotion 43

2.5 Example of coefficients for the probability distribution . . . . . . 43

3.1 Circular mean and circular standard deviation in degress for each emotion in Portuguese, Swedish, and Spanish languages. . . . . .

3.2 Results of the chi-square test for the comparison of the distribution of emotions across languages. Note that $*$ indicates p-value $<0.05 .79$

3.3 Example of the results of the Emotion Representation process for the emotion "Happiness" with a Medium intensity. . . . . . . . . 81

$3.4 \mu_{A^{i}}(u)$ values for the example $(u=0.625) \ldots \ldots . . \ldots . . . .83$

3.5 Example of $\mu_{B^{j}}(\alpha)$ values for $\alpha=245^{\circ}$. For the Portuguese and Swedish labels see Table 3.1. . . . . . . . . . . . . . . . . . 83

3.6 Results of the chi-square test for the study of the influence of gender and age on the assignment of pleasure and arousal values for the ten emotions in the Portuguese and Swedish samples. Note

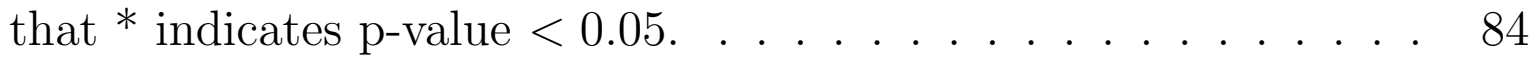

4.1 Fuzzy model for estimating the emotion and its intensity . . . . . 108

4.2 Results of the experiment expressed in degrees. . . . . . . . . . 111

4.3 Example of $\theta_{e, p}$ values. . . . . . . . . . . . . . . . . . . . . 114

5.1 Example of a personality / emotion correlation matrix. . . . . . . 149 


\section{Part I}

\section{Introduction and objectives}



Chapter 1

\section{Introduction and objectives}

"The heart has its reasons which reason knows not of" - Cite associated with the French mathematician, physicist, and philosopher Bleis Pascal [162]. 
Today's society is increasingly moving towards the use of computer systems and virtual environments. Human-machine interaction has become an everyday phenomenon in both personal life and workplace. In recent years, a special effort has been made to develop systems capable of simulating more natural human-like interactions [40, 115]. Generally, the approaches proposed in this area are based on the agent-oriented paradigm. Software agents are able to reason, act proactively, and cooperate with other agents to establish plans that allow them to achieve their objectives [28]. Traditionally, most of the proposals using the agent-oriented paradigm are based on some types of logic and economic theory, generally through the maximization or minimization of an utility function, to carry out the agent's action planning. One of the best known model that allows the development of "rational" agents is the BDI (beliefs-desires-intentions) model that is based on Aristotelian practical reasoning. This type of practical reasoning is based on the intention to achieve a goal and a reasoned decision-making process performed to select an action (or plan of actions) from a set of possible actions to achieve that goal [225]. Over the years, several agent models capable of simulating the human "logical reasoning" process in controlled environments have been proposed. However, this type of agents based on classical practical reasoning present some limitations regarding the system's capacity to simulate certain human behaviors. This is because human behavior does not always respond to logic but is often guided by emotions and other affective, intrapersonal, or interpersonal factors. There are several studies from different fields of psychology, sociology, ethology, and neuroscience that relate diverse aspects of affect (e.g., emotions, mood, or personality) with the capacity for reasoning, judgment, decision-making, or risk tolerance (among others) [8, 80, 240]. For example, empirical evidence suggests that there is a critical impact of emotions on cognition and a high variability of this impact among individuals with different personality factors. This is because personality can make a person more or less likely to experience certain types of mood [33, 133]. Therefore, one of the most significant challenges that the research community on human simulation and human-machine interaction faces, is to succeed in simulating affective and social human behavior through credible intelligent agents or virtual characters as well as system personalization, i.e., generating systems capable of 
been adapted to different user characteristics to offer a customized experience $[37,42,241]$. In order to simulate this type of "non-logical rational" behavior through intelligent agents, it is necessary to consider the factors related to social interaction, social cognition, intrapersonal or interpersonal characteristics, affective state, or social context (among others) when establishing the agent's reasoning, decision-making, or behavior selection mechanisms. However, systems capable of simulating human behavior at a social and affective level are complex and require high level abstractions and metaphors to describe capabilities and characteristics that cannot be explained by classical low level models. These metaphors and abstractions are aimed at simulating human behaviors with a higher level of realism as well as reflecting more closely the way in which humans understand the world. In this sense, over the years, in the field of affective computing [170], different models of agents capable of recognizing, processing, and simulating different abilities related to human affective behavior have been proposed [128]. These models typically include different cognitive components such as affective states or personality that come from different branches of psychology, ethology, philosophy, and sociology. Affective states, which encompass emotions and moods, are complex constructs whose definition remains the subject of debate to this day. Although the difference between emotions and mood continues to be a topic of debate [19], in general, it is accepted that an emotion is a rapid response to a given stimulus, while mood has a longer duration (from minutes to days) and a lower intensity than emotions and is not related to any particular stimulus. Instead, mood is produced by a succession of stimuli and other factors such as the context or the person's personality [64].

Over the years, several theories have been proposed in an attempt to explain how affective states are generated in the human brain. One of the most popular theories is the appraisal theory $[72,163]$. According to this theory, when a stimulus is perceived, an appraisal process is produced, resulting in an emotion [135]. These theories are based on the existence of different variables known as appraisal variables [231], whose number and type vary depending on the author. Therefore, it is necessary to determine the mechanisms associated with these appraisal theories and how to translate these mechanisms into agent model [182]. 
On the other hand, there are several theories that study the representation of affective states in the human brain as well as the processes that are related to them. In this sense, there are several theories of representation of affective states that can be generally classified into two main groups: categorical and dimensional [93]. On the one hand, categorical approaches are based on the use of a limited set of words or labels to define the different affective states [66]. These theories are based on how humans express and recognize complex cognitive concepts, which represent affective states, through the use of different words such as "sadness" or "joy". This way of representation is commonly used in the area of affective computing to generate models for recognition, interpretation, processing, and simulation of affective states $[17$, 20, 74]. These words provide a means to verbalize affective states, which is a key factor in human affective interactions. One of the best known models is the model of basic emotions proposed by P. Ekman in which six labels are defined: sadness, happiness, anger, fear, disgust, and surprise [65]. This model is one of the most widely used in the area of affective computing to express, recognize, and represent affective states [20, 173].

In contrast, dimensional approaches allow to reduce the level of abstraction by representing affective states through different dimensions. These theories have their origin in psychological constructivism, according to which, affective states cannot be established by a finite set of labels since they depend on the individual's subjective experience that occurs in a particular context (e.g., a particular culture or language). One of the most representative dimensional models is the Core Affect model proposed by J.A. Russell [194]. In that model, affective experiences are defined as emotional cues and are represented by two dimensions: pleasure and arousal. Russell also evidenced through experimentation that the labels used to define the different affective states followed a circular pattern along these two dimensions. From these results he developed the Circumplex Model of Affect [174] that has also been used in affective computing [204, 243].

Moreover, several studies over the years have shown that emotions have a high dependence on language and culture $[23,46,196]$. The same word or label used to define an emotion or affective state can have a different conceptualization and be interpreted in different ways depending on the cultural 
environment. In fact, there are words that express affective states that are very difficult to translate into other languages $[107,137]$. Therefore, to establish generic models of affect representation capable of adapting to different cultural environments, it is necessary to establish methodologies that allow to adapt these models to other languages and cultures.

In recent years there is a growing trend towards the development of agents with skills related to social cognition $[142,165,236]$. Social cognition is a construct used to encompass the cognitive processes that underlie the development of social interactions and relationships, including phenomena such as social perception, Theory of Mind, and empathy. Social perception refers to the cognitive ability of evaluating the social context (e.g., norms, rules, or relationships) in order to make judgments about other individuals involved in that social context [77, 228]. Theory of Mind (also referred to as perspectivetaking) is a cognitive ability that allows one to understand and predict the mental states, behaviors, beliefs, and intentions of others [226]. Theory of Mind is a fundamental component in the development of other high level cognitive and social skills such as empathy. Finally, empathy is a construct that encompasses different processes aimed at understanding and feeling the affective state of others $[22,50]$. Several authors consider empathy as an inherent factor in the development of the human being as a social individual $[22,100,184,157,205]$. Empathy also plays a fundamental role in our society, affecting both morality and mutual understanding. [99]. In fact, empathy appears to be the basis on which social behaviors and interpersonal interactions are founded [50, 149]. Empathy connects people by promoting relationships and collaborations through the exchange of experiences, needs, and desires. [184]. However, the definition of the term empathy continues to divide the academic community [164]. This is largely due to the fact that the concept of empathy encompasses a large number of emotional, ethical, moral, and social factors. This lack of consensus can be clearly seen in the review conducted in [49], in which the authors identified forty-three different definitions for the concept of empathy.

For all the above mentioned reasons, empathy is postulated as a fundamental piece in the development of models focused on the simulation of human social behavior as well as in the improvement of human-machine interaction 


\subsection{MOTIVATION}

[233]. In fact, systems that are capable of simulating some level of empathic abilities have been used in different contexts and have proved to be more reliable and more credible, thus reducing the stress and frustration of human users and improving human-machine interaction [165]. However, this type of models are generally presented as ad-hoc proposals for a predetermined scenario and with a limited capacity to simulate emotional, social, or empathic behavior $[32,138,189]$. Therefore, much work remains to be done in the areas of affective computing, simulation of human behavior, and human-machine interaction until a reliable simulation of empathic interactions and human behavior will be achieved.

\subsection{Motivation}

In recent years we have experienced an exciting evolution in the field of artificial intelligence and affective computing. However, we are still far from being able to understand and reliably simulate the cognitive processes that occur in the human brain and that constitute the foundation of human behavior. One of the remaining challenges is to generate models to simulate human social and affective behavior. Motivated by this challenge, at the beginning of this thesis we proposed the design of an affective agent model capable of simulating empathic interactions through an automatic reasoning process that would allow to adapt the agent to different cultural environments, contexts, and social situations.

One of the first difficulties that appear in this type of system is the way in which the agent's knowledge is represented. For a software agent to be able to reason using abstract concepts such as emotions, mood, or empathy, it is necessary to study how these constructs are produced and verbalized. If we pay attention to the usual way in which human beings refer to emotions or moods we observe that we generally use terms such as "very sad" or "happy". Therefore, it is necessary for the system to be able to recognize this terminology and apply it in its affective reasoning system. This can be achieved by a model of affective states representation that will allow the agent to recognize and use this terminology as well as to apply it in its affective reasoning 
process.

There are several models of representation of affective states that are commonly used in the field of affective computing. Categorical models, such as Ekman's basic emotions model [66], provide a simple way of representing affective states by means of a finite set of affective words or labels. However, by a label based representation we are assuming a high level of abstraction since we are condensing the entire spectrum of affective states into a limited set of labels. Therefore, we are losing part of the affective information, such as the intensity of the affective state, or the probability of moving from one affective state to another depending on a certain stimulus [71, 163, 232]. In addition, the use of these words to define affective and cognitive terms is subject to possible variations between different cultures and languages $[151,196]$. For example, the German vocabulary has an extensive catalog of affective labels defining emotions such as Schadenfreude which can be summarized as "pleasure from the suffering of others" or Sehnsucht which can be described as "a strong desire for an alternative life" but whose overall conceptualization is very difficult to describe by means of a word or label in other languages such as English or Spanish [107, 137]. Therefore, translating emotional words or labels from one language to another can lead to cultural misunderstandings as well as undesired behaviors that could harm the simulation of affective behavior and the user's experience.

On the other hand, dimensional approaches, such as the Circumplex Model of Affect, propose a representation in a continuous space based on a limited set of dimensions. However, the direct use of this model in affective computing, presents a number of disadvantages probably because it was not designed for its use in a computational system. The first disadvantage is evident from the way in which emotions are represented by a single point in the pleasurearousal space. This point-based representation makes it difficult to explore the relationship between the dispersion of emotions and the levels of pleasure and arousal and is far from the way in which emotions are understood by humans, as we prove in the experiment presented in Section 2.3. Another clear disadvantage is that that model does not consider the relationship between the dimensions of pleasure and arousal and the intensity of the emotions. Finally, it is necessary to consider the cultural dependence of the model, i.e., the 
model was designed from an experiment with English-speaking participants and therefore a direct application to other languages may not be appropriate.

On the other hand, in the development of models to simulate human affective behavior, it is necessary to establish mechanisms to allow the agents to simulate the suitable emotions according to the perceived stimulus or event. In that sense, one of the most relevant theories to explain the phenomenon of emotion in the field of psychology is the appraisal theory. In the literature we find several models of affective appraisal. However, most of the models used in affective computing use numerical variables, which is far from the way humans interpret cognitive states $[81,188]$. Moreover, to our knowledge, current computational appraisal models do not allow the elicitation of emotions with intensity. However, the intensity of affective states is an important part of human affective behavior.

\subsection{Objectives}

Considering the motivations described in Section 1.1 the main objective of the research process conducted in this thesis is the development of a formal model of an affective agent capable of simulating empathic interactions (both with human users and software agents), of expressing its affective states using the same terms used by human beings, and of adapting its behavior to different cultural environments. In order to achieve this goal it is necessary to develop (i) a model for representing and expressing affective states, (ii) a methodology to adapt this representation model to different cultural environments, (iii) an appraisal process to elicit emotions in affective agents, and (iv) a formal definition of an affective agent that use the appraisal and the representation of affective states to simulate empathic interactions. Therefore, the following specific objectives are proposed:

1. Review of the state of the art.

1.1 Analyze the theories related to affective states, affective state dimensional representation and cultural difference, emotion appraisal, 
and empathy from the perspectives of psychology, neuroscience, sociology, and computating.

1.2 Analyze of the state of the art related to the development of affective agents focusing on the approaches that consider empathic behaviors.

2. Design a cross cultural computational model for affective state representation

2.1 Determine the structure of a representational model considering current theories in the fields of psychology and neuroscience.

2.2 Design of a method to discover the structure of affective states in the representation model.

2.3 Conduct an experiment with humans to define the representation model

2.4 Replicate the experiments in other cultural environments to validate the experiment-based methodology and adapt the model to other cultures and languages.

3. Define a human-like model to elicit and express affective states.

3.1 Develop a computational model of appraisal based on fuzzy logic to use human-like affective terminology.

3.2 Define how affective states and personality influence the rational processes of an agent.

3.3 Define a set of fuzzy rules to represent and express affective states using a human-like affective terminology.

4. Design a formal model for affective agents with empathic abilities.

4.1 Design the internal processes for recognizing and representing perceived affective states and to allow the emergence of empathic emotions in an affective agent 
4.2 Design the processes to adapt empathic emotions to different intrapersonal and interpersonal characteristics to simulate the empathic regulation.

4.3 Integrate this model in the general-purpose architecture GenIA ${ }^{3}$.

5. Propose a case of study to validate the formal design of the effective agent with empathic abilities.

\subsection{Structure of the thesis}

In this part we have provided an introduction to the work proposed in this thesis. Starting from the motivations, an overview of the challenges of the thesis have been provided. Then, the objectives of the research have been enumerated. The rest of the document is organized as follows:

- Part II Contribution: In the second part of this document the most representative contributions supporting the objectives of this thesis are presented. Following, a brief summary of each chapter is provided:

Chapter 2 (Selected paper) proposes an experiment-based methodology for adapting a pleasure and arousal representation space for affective computational simulation and recognition to different cultural environments. In addition, the results of an experiment conducted with European Spanish-speakers are presented. These results are then used to provide a new cross-cultural model based on fuzzy logic to represent affective states in the pleasure and arousal dimensions using a polar coordinate approach.

Chapter 3 shows the results of an experiment conducted in collaboration with the ALGORITMI Centre, University of Minho (Portugal) and the Department of Computing Science, Umeå University (Sweden) to adapt the computational representation model presented in Chapter 2 to Portuguese and Swedish. The results are 
compared with the experiment conducted with European Spanishspeakers, presented in Chapter 2, to prove that cultural differences affect the pleasure and arousal values associated with each emotion.

Chapter 4 (Selected paper) presents a new fuzzy appraisal model for eliciting emotions in affective agents. The emotions generated in the agent by the fuzzy appraisal rules are expressed in a pleasure an arousal representation based on the work presented in Chapters 2 and 3. Moreover, we present an affect regulation process that adapts the agent's mood, represented by a vector in the pleasure-arousal space, each time an emotion is elicited.

Chapter 5 describes a formalization of the syntax, the semantics, and the reasoning cycle of AgentSpeak to allow the development of affective agents with empathic abilities. Based on the theories of empathic appraisal and empathic regulation, this formalization departs from the General-purpose Architecture for Affective Agents GenIA ${ }^{3}$ and modifies the agent's reasoning process to allow empathy to affect its decision-making process. An empathic and an affective appraisal process, based on the proposal made in Chapter 4, allows the agent's to elicit empathic emotions when perceiving a situation in other agents. The empthic regulation process adapts the elicited empathic emotion to different intrapersonal and interpersonal factors. The agent is also able to maintain the distinction between the self and the others.

Chapter 6 (Selected paper) introduces an application of the empathic agent model proposed in Chapter 5. This application is based on an empathic pedagogical agent model for education on good practices in the use of social networks. The agent is able to recognize the user emotions when interacting with the social network. Based on the user emotions and his/her behavior on the social network, the agent estimates the best plan to teach the user in the correct and secure use of social networks. 
- Part III Discussion: In this part a discussions of the results obtained during the research period of this thesis is presented.

- Part IV Conclusions and Future Work: Finally, this last part shows the conclusions of the research conducted in this thesis and propose possible future works.

\subsection{List of publications}

The work done in this thesis is supported through the following publications (note that publications marked with * are those included in this thesis document):

- Journals listed in JCR:

- *Joaquin Taverner, Emilio Vivancos, and Vicente Botti. A multidimensional culturally adapted representation of emotions for affective computational simulation and recognition. IEEE Transactions on Affective Computing, doi: 10.1109/TAFFC.2020.3030586. Impact Factor: 10.506.

- *Joaquin Taverner, Emilio Vivancos, and Vicente Botti. A fuzzy appraisal model for affective agents adapted to cultural environments using the pleasure and arousal dimensions. Information Sciences, 546:74-86, 2021, doi: 10.1016/j.ins.2020.08.006. Impact Factor: 6.795.

- Bexy Alfonso, Joaquin Taverner, Emilio Vivancos, and Vicente Botti. From Affect Theoretical Foundations to Computational Models of Intelligent Affective Agents. Applied Sciences, no. 22: 10874, 2021, doi: 10.3390/app112210874. Impact Factor: 2.679.

- International conferences listed in CORE:

- Joaquin Taverner, Bexy Alfonso, Emilio Vivancos, and Vicente Botti. Modeling personality in the affective agent architecture Ge- 
nIA3. In Int. Conf. on Agents and Artificial Intelligence, pages 236-243, 2018. Doi: 10.5220/0006597202360243.

- Joaquin Taverner, Emilio Vivancos, and Vicente Botti. Towards a computational approach to emotion elicitation in affective agents. In International Conference on Agents and Artificial Intelligence, pages 275-280, 2019. Doi: 10.5220/0007579302750280.

- Jose Manuel Fuentes, Joaquin Taverner, Jaime Andres Rincon, and Vicente Botti. Towards a classifier to recognize emotions using voice to improve recommendations. In International Conference on Practical Applications of Agents and Multi-Agent Systems, pages 218-225. Springer, 2020. Doi: 10.1007/978-3-030-51999-5_18.

- *Joaquin Taverner, Emilio Vivancos, and Vicente Botti. Towards a model of empathic pedagogical agent for educating children and teenagers on good practices in the use of social networks. In Int. Conf. on Agents and Artificial Intelligence, pages 439-445, 2021. Doi: 10.5220/0010345504390445.

- Andreas Brännström, Timotheus Kampik, Ramon Ruiz-Dolz, and Joaquin Taverner. A Formal Framework for Designing Boundedly Rational Agents. In Int. Conf. on Agents and Artificial Intelligence, (Accepted), 2022.

- Other international conferences:

- Joaquin Taverner, Bexy Alfonso, Emilio Vivancos, and Vicente Botti. Using different agent personalities to obtain different agent behaviours. In Proc. of the Workshop on Affective Computing and Context Awareness in Ambient Intelligence, 2018.

- Joaquin Taverner, Ramon Ruiz, Elena del Val, Carlos Diez, and Jose Alemany. Image analysis for privacy assessment in social networks. In International Symposium on Distributed Computing and Artificial Intelligence, pages 1-4. Springer, 2018. Doi: 10.1007/978-3-030-00524-5_1. 
- Joaquin Taverner, Emilio Vivancos, Vicente Botti, and Bexy Alfonso. Influence of the agent personality on its mood. In Proc. of the Workshop on Affective Computing and Context Awareness in Ambient Intelligence, 2019.

\subsection{List of projects}

- FPI grant

- Funder: Generalitat Valenciana and European Social Fund (ACIF/ 2017/085).

- Lead Applicant: Joaquin Taverner Aparicio.

- Years: 2017 - 2021.

- Intelligent Agents for Privacy Advice in Social Networks (AI4PRI).

- Funder: Ministerio de Economia y Empresa (TIN2017-89156-R).

- Lead Applicant: E. Argente, and A. García-Fornes.

- Years: 2018 - 2021.

- Affective Intelligent Agents for Persuading Civic Behavior in Virtual Environments

- Funder: Ministerio de Economia y Empresa (PID2020-113416RBI00).

- Lead Applicant: E. Argente, and A. García-Fornes.

- Years: 2021 - 2024. 


\section{Part II}

\section{Contribution}





\title{
Chapter 2
}

\section{A multidimensional culturally adapted representation of emotions for affective computational simulation and recognition}

\author{
Joaquin Taverner, Emilio Vivancos, and Vicente Botti \\ IEEE Transactions on Affective Computing \\ Doi: https://doi.org/10.1109/TAFFC.2020.3030586
}

\begin{abstract}
One of the main challenges in affective computing is the development of models to represent the information that is inherent to emotions. It is necessary to consider that the terms used by humans to name emotions depend on the culture and language used. This paper presents an experiment-based method to represent and adapt emotion terms to different cultural environments. We propose using circular boxplots to analyze the distribution of emotions in the Pleasure-Arousal space. From the results of this analysis, we define a new cross-cultural representation model of emotions in which each emotion term is assigned to an area in the Pleasure-Arousal space. An emotion is represented by a vector in which the direction indicates the type, and the module indicates the intensity of the emotion. We propose two methods based on fuzzy logic to represent and express emotions: the emotion representation process in which the term associated with the recognized emotion is defuzzified and projected as a vector in the Pleasure-Arousal space; and the emotion expression process in which a fuzzification of the vector is produced, generating a fuzzy emotion term that is adapted to the culture and language in which the emotion will be used.
\end{abstract}




\subsection{INTRODUCTION}

\section{$2.1 \quad$ Introduction}

Emotions play an important role in our behavior as humans. Emotions are present in our day-to-day relationships with other human beings and when we face any situation or event. Systems that are capable of recognizing, processing, or simulating emotions can improve the interaction between humans and machines by making this interaction more natural and realistic.

Over the years, different models have been proposed to recognize emotions in human beings. With the improvement of machine learning, there is currently a large number of models that recognize emotions using images, voice, text, or electroencephalograms. However, most of these models focus on improving the accuracy of the recognition without paying much attention to the way of representing that knowledge for its possible use in systems such as affective agents. An affective agent is a system that is capable of simulating human affective behaviour. Currently, there are several approaches for affective agents in which different models of representation of emotions are used [6, 81, 148, 155, 182]. Frequently, proposals made from affective computing represent emotions using simple labels (e.g., Happy or Sad). However, a representation of emotions that uses a continuous multidimensional space seems to be more appropriate for use in computational models since it allows storing more information about emotions, such as the intensity of emotions or the proximity between emotions [34]. This representation also provides a greater capacity to analyze the variations that occur in emotions and mood over time, thereby improving the simulation of human emotional behavior.

Different models of representation of emotions have been proposed from psychology. One of the best known is The Circumplex Model of Affect proposed by J. A. Russell [193] (Fig. 2.1). This model relates emotions with their Pleasure and Arousal values. In addition, according to several studies, emotions greatly depend on language and culture [196, 23, 46]. In other words, the same emotion label can be interpreted in a different way depending on the culture and the language. Nevertheless, even though cultural and language factors are taken into account in other domains such as recommendation systems, to our knowledge, there are still no proposals in the affective computing area that really consider cultural and language factors to represent 


\section{CHAPTER 2. A CULTURALLY ADAPTED REPRESENTATION OF EMOTIONS (SELECTED PAPER)}

and express emotions in a computational system.

On the other hand, there is a recent tendency to use emotional models based on fuzzy logic $[150,13]$. This is due to the fact that this type of knowledge representation is closer to the way in which human beings express their emotions using terms such as very happy or a little happy [122]. Therefore, a model that represents human emotions should be able to deal with this type of fuzzy terminology.

This paper proposes an experiment-based method that represents emotions in a Pleasure-Arousal dimensional space adapted to the language and culture where the emotions will be expressed. This model uses fuzzy logic to better simulate the way in which humans express emotions. The rest of this paper is organized as follow. In Section 2.2, we discuss the most important proposals for emotion representation in affective computing. Section 2.3 introduces an experiment-based method to adapt the emotion representation to the cultural environment in which emotions are used. The fuzzy culturally adapted model of affect obtained from the experiment is presented in Section 2.4. Finally, the main conclusions and some future works are presented in Section 2.5.

\subsection{Related works}

The term emotion is a psychological construct whose definition has long been the subject of debate. Over the years, different theories have been proposed to explain emotional behavior. Some theories, such as basic emotion theories, propose that there is a limited number of emotions and that these emotions are understood universally. One of the best known theories in this area is the Basic Emotion Theory proposed by Ekman [65]. Ekman's theory is based on six basic emotions: Happiness, Surprise, Fear, Anger, Disgust, and Sadness. Plutchik defined his theory using eight basic emotions [172] in which different feelings can be derived from the combination of two adjacent emotions (Fig. 2.2). According to these theories, one event is universally related to a single emotion.

In contrast, the constructivist theories contradict the basic emotions theories stating that emotion labels/words do not have a universal meaning. 


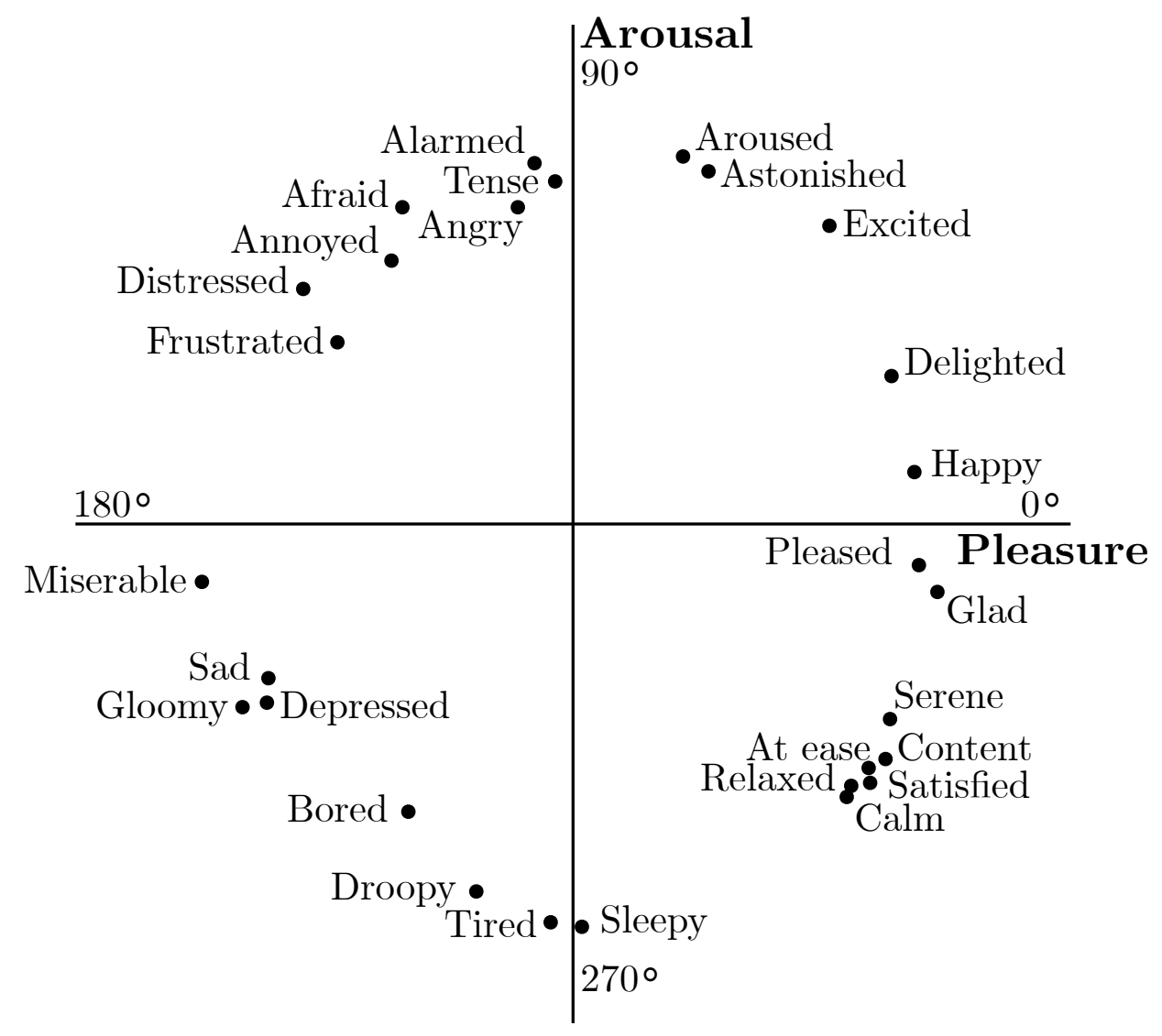

Figure 2.1: The Circumplex Model of Affect

According to constructivism, the labels that we use to refer to emotions depend on the culture and the language used. Therefore, there is no direct correspondence for all of the emotion labels among different languages [97]. For example, in the German language, there is an emotion called Schadenfreude, whose meaning could be translated into English as "pleasure from the suffering of others" but this translation does not quite capture the complete meaning of the emotion as it is shown in [137].

One of the most cited constructivist theorists is J. A. Russell. In his CoreAffect theory [196] language and culture have an important weight when cataloging different emotions. He also proposed that emotions are related to the dimensions of Pleasure (sometimes called Valence) and Arousal (sometimes called Activation) [194]. He observed through experimentation that emotions follow a circular pattern within a continuous space based on these 


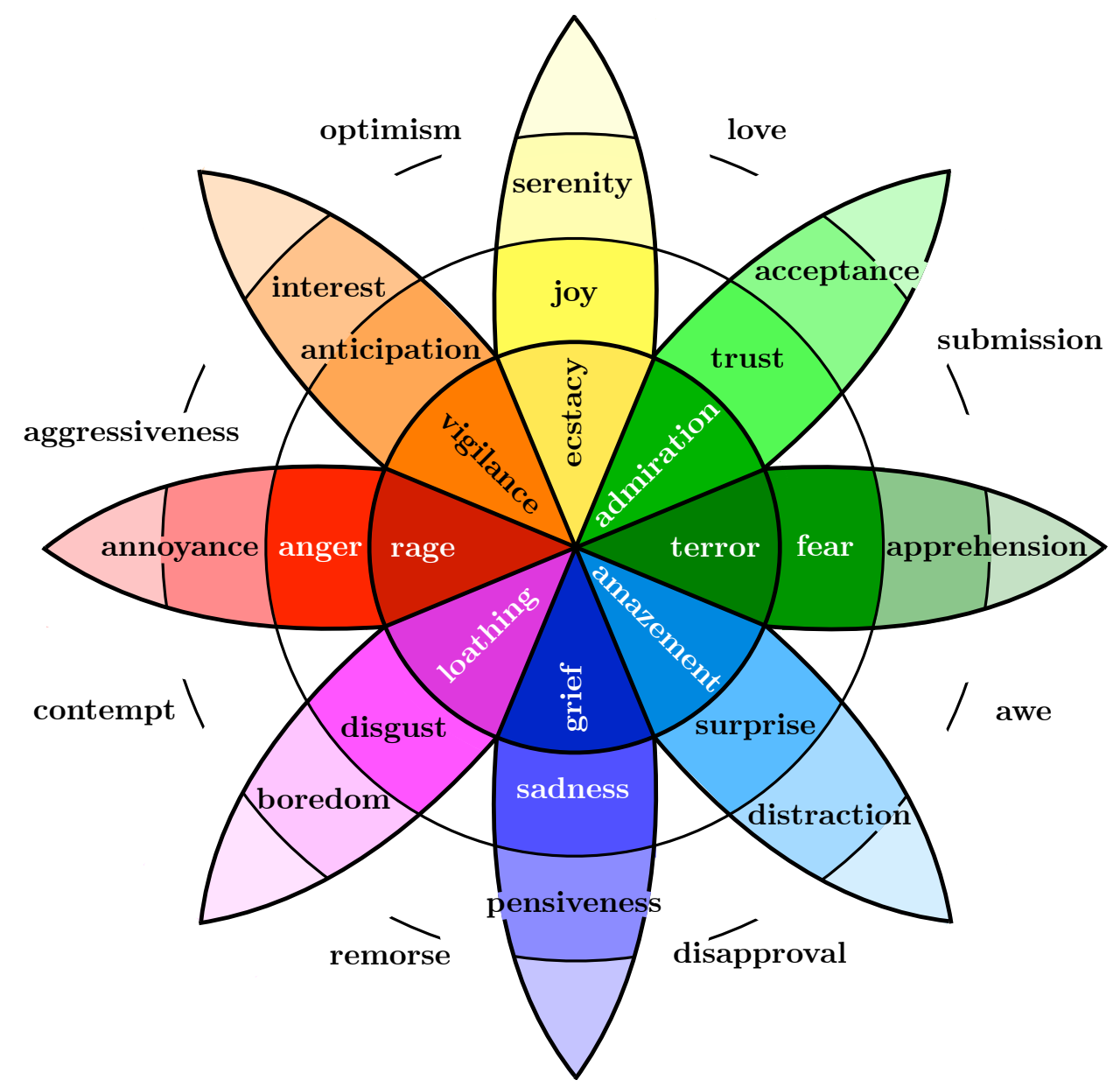

Figure 2.2: Wheel of emotions proposed by Plutchik [172]

two dimensions. He called this pattern The Circumplex Model Of Affect [193] (Fig. 2.1), which shows the relationship between emotions and the values of Pleasure and Arousal.

On the other hand, Reisenzein [183] observed that the intensity of emotions is proportionally related to the values of Pleasure and Arousal: the greater the value of these dimensions, the greater the intensity of the emotion. For example, the intensity of the Happy emotion is more related to the level of Pleasure, while the intensity of the Alert emotion is more related to the level of Arousal. In addition, he also observed that low levels of Pleasure and Arousal mean that the intensity of emotions is so low that it can be assumed that there is no emotion. 


\subsection{RELATED WORKS}

Mehrabian proposed adding a third dimension, known as Dominance, to the Pleasure-Arousal space. This created a new model known as the PAD model [154], which is one of the most widely used models when designing affective agents that simulate emotional behavior. The Dominance dimension increases the space of representation allowing some emotions to be disambiguated. However, many models propose that this third dimension must be considered as an appraisal variable rather than as a representation variable [147].

Jackson et al. [107] analysed around 2500 languages to determine the level of similarity of 24 emotion labels across different cultures. They studied six dimensions associated with emotions (Pleasure, Arousal, Dominance, Certainty, Approach-avoidance, and Sociality) and detected that Pleasure and Arousal are the two most important dimensions to determine emotion semantics across different languages. In fact they concluded that Pleasure and Arousal are "common psychophysiological dimensions shared by all humans". Other studies showing that the behavior of emotions in the Pleasure-Arousal space depends on culture and language have been conducted over the years [196, 237]. Based on these findings, different psychology researchers have adapted some psychological models to different cultures and languages [57].

\section{Emotions in Affective Computing}

Currently, most of the proposals made in the affective computing area, both in the field of recognition and simulation, use Ekman's emotional model to define emotions $[242,81]$. However, there are other models that use a dimensional representation of emotions [146, 13, 216]. For instance, the World Wide Web Consortium (W3C) recommends a markup language that allows both dimensional and label-based representations of emotions (EmotionML [203]).

Generally, the proposals performed in the field of affective behavior simulation are focused on the use of intelligent multi-agent systems [185, 199]. Most of these proposals take advantage of a dimensional representation to model different affective processes, such as the mood decay rate or the displacement of the mood when receiving an emotion, which would be impossible 


\section{CHAPTER 2. A CULTURALLY ADAPTED REPRESENTATION OF EMOTIONS (SELECTED PAPER)}

to simulate using a label-based representation. For example, the model proposed by Gebhard in ALMA (A Layered Model of Affect) [81] uses the PAD model to represent the mood in an affective intelligent agent. On the other hand, GenIA ${ }^{3}$ (a general purpose architecture for affective agentes) [6] is a BDI architecture created to simulate affective behavior in multi-agent systems. GenIA ${ }^{3}$ offers a set of tools to represent affective traits like personality, emotions, and mood. This architecture also uses a dimensional representation for mood and emotions. However, these models assume that a basic emotion can be universally represented by a simple point in the dimensional space. The work proposed in this paper extends the GenI $A^{3}$ architecture through a new emotional representation model to adapt $G e n I A^{3}$ affective agents to different cultures and languages.

In recent years, there seems to be a tendency to define affective models using fuzzy logic. Through fuzzy logic, it is possible to approximate the artificial representation of emotions to the way in which humans express them. For example, Jain et al. propose EMIA [108] which uses fuzzy rules to handle uncertainty in its appraisal process for five of Ekman's basic emotions. Another interesting proposal is the one presented in [121] in which a methodology to translate emotional labels from Spanish to English through fuzzy logic is proposed. The author designed an experiment in which 8 Spanishspeakers answered a questionnaire to obtain the values of Pleasure, Arousal, and Dominance associated with 30 Spanish emotional labels/words. From the results of the experiment, the fuzzy membership functions for each emotion were obtained. Then a comparison with the functions obtained with Englishspeakers in a previous experiment was performed to translate the emotional labels/words.

As the Circumplex Model of Affect shows, emotions follow a circular pattern in the Pleasure-Arousal space. This property suggests that emotions could be represented by the use of vectors in a polar coordinate system. For example, in [106], a vector based representation model of emotions using Pleasure and Arousal dimensions is proposed. The authors modify the representation space by displacing the origin of coordinates to the negative extreme of the Arousal dimension. However, when displacing the origin of coordinates, both the direction and the modulus of the vector are modified, which 
can lead to difficulties when calculating the intensity or type of the emotion. In addition, the concept of negative Arousal (usually referred to as Sleepiness or Deactivation) becomes blurred, producing a decontextualization of emotions that depend on this dimension, such as Boredom or Calm. These disadvantages can be solved by the use of circular statistics.

When using circular representation spaces, such as the Circumplex Model of Affect, traditional statistics that are focused on linear data may not be adequate since the representation space is finite and constrained between 0 and $2 \pi$. In a circular representation space based on Pleasure and Arousal, the dispersion can be estimated by the circular standard deviation $\sigma$ expressed as follows [169]:

$$
\sigma=\sqrt{-2 \ln \bar{R}}
$$

where

$$
\bar{R}=\sqrt{\bar{P}^{2}+\bar{A}^{2}}
$$

where $\bar{P}$ and $\bar{A}$ represent the mean values for the Pleasure and Arousal dimensions. The distribution of the data in a circular area delimited by $2 \pi$ also makes it difficult to use the probability distributions that are commonly used in linear statistics. Instead, the von Mises distribution [102] could be used to estimate the normal (Gaussian) distribution for circular data. For example, in [92], a probabilistic model based on a mixture of von Mises distributions for the recognition of emotions in faces is proposed. The authors use a representation space that is based on the dimensions of Pleasure and Arousal. The face images were projected to coordinates in the representation space to determine the type of emotion using the von Mises distribution.

\section{Discussion}

Despite the great amount of effort made to recognize and simulate emotions, there is still much to be done until we are able to understand and correctly simulate affective processes. Nowadays, there is no consensus about what is 


\section{CHAPTER 2. A CULTURALLY ADAPTED REPRESENTATION OF EMOTIONS (SELECTED PAPER)}

the best way to represent emotions. This is because emotions are complex concepts and their generation depends on multiple personal, contextual, and cultural factors. However, in order to make better use of the current systems for emotion recognition and affect simulation, it is necessary to design a computational model of affect that represents this knowledge as flexibly and accurately as possible. A common representation using a continuous dimensional space for both recognized and simulated emotions can improve the understanding and simulation of affective processes such as emotion elicitation, emotional contagion, or empathy.

However, in affective computing, the use of emotions is usually simplified by using categorical approaches that reduce emotions to simple labels (e.g., using the six basic emotions of Ekman). With this type of representation, part of the information inherent to the emotion, such as intensity or proximity to another emotion, is lost. Therefore, representations of emotional knowledge based on a multidimensional continuous space are more appropriate for use in affective computing. In a multidimensional space, emotions can move through continuous variables representing a greater amount of information (e.g., the intensity of the emotion) than if only labels were used.

When a dimensional representation is used, different alternatives for representing the emotions within the space arise. The most commonly used model consists of representing each basic emotion as a point in the representation space as is done in The Circumplex Model of Affect (Fig. 2.1). The main problem that appears when using this model is the way basic emotions are represented using a simple point in the space. This representation is far from the way in which emotions are associated with Pleasure and Arousal by humans, as we prove in our experiment. The second problem when using this scheme is the ambiguity that appears in the spaces between adjacent emotions, because the area around each point is not clearly defined. In addition, it is very difficult to determine categorically and without error that a point in space corresponds to a certain emotion. Moreover, this model does not represent important information such as the intensity of the emotion. Therefore, a more appropriate method for representing the emotions consists of defining each emotion as an area in the emotion space, instead of a simple point as proposed by other approaches. An area-based method better represents the 


\subsection{AN EXPERIMENT-BASED METHOD TO ADAPT AFFECTIVE MODELS TO CULTURE AND LANGUAGE}

complexity and similarities among multiple emotions.

Some findings suggest that languages around the world primarily differentiate emotions on the basis of Pleasure and Arousal [107]. Therefore, when defining a computational model of affect, the culture and the language in which it is going to be used must be considered since different cultures may attribute different Pleasure and Arousal values for the same emotion label [46, 141]. For example, in the experiment presented in [196], the authors note, among other results, that the Polish participants related the calm emotion with a significantly higher level of arousal than the Greeks. Extrapolating an emotional model from one language to another without adapting it to the culture and language may produce inconsistencies, which can lead to erratic agent behavior and emotion expression. Moreover, the way in which emotions are expressed when the system interacts with humans must also be taken into account. Fuzzy logic allows affective agents to express their emotions in a similar way to humans.

\subsection{An experiment-based method to adapt affective models to culture and language}

Labels that are used to represent emotions do not have the same meaning in different languages and cultures, but a model that is based on the Pleasure and Arousal dimensions can represent the universal meaning of the emotions [107]. Nevertheless, the scheme proposed by Russell is a basic scheme that is used to support the circular representation of emotions. Therefore, the direct use of this scheme in a computational model can easily be criticized since it was not defined for that purpose. In addition, this scheme was generated from experiments with English-speakers. Therefore, following the foundations of constructivist theories, this representation of basic emotions may not be valid in other cultures or languages. It is necessary to design processes to adapt this emotional model to the culture and language of the environment in which the model will be used and also to discover the areas of the multidimensional space where emotions have a higher probability of occurrence. We present an 


\section{CHAPTER 2. A CULTURALLY ADAPTED REPRESENTATION OF EMOTIONS (SELECTED PAPER)}

experiment-based method to find the regions representing each emotion, that can be used for different languages and cultures.

We start from the main hypothesis that Russell's model might not be valid for representing emotions in a context with a language and a culture that is different from the context in which Russell carried out his experiment. We propose a methodology that consists of two experiments. The first experiment finds the labels that best represent the meaning of the Pleasure and Arousal dimensions in the target language. In our experiment the target language is European Spanish. The second experiment finds the intervals of Pleasure and Arousal that are associated to each emotion in the target language.

For our experiment, we have selected the ten emotions used by Russell in [196]: Fear, Anger, Disgust, Sadness, Boredom, Sleepiness, Calm, Happiness, Excitement, and Surprise. The most common literal translations of these emotions into Spanish are "Miedo", "Enfado", "Asco", "Tristeza", "Aburrimiento", "Somnolencia", "Calma", "Felicidad", "Emoción", and "Sorpresa". However, these are not the only possible translations, and, depending on the selected word, we could be expressing different emotional meanings. Therefore, making a literal translation of emotions could lead to significant differences in the interpretation of the levels of Pleasure and Arousal of these emotions.

\section{Method}

\section{Experiment 1: Naming the Pleasure and Arousal dimensions}

Starting from the hypothesis that the literal translation of the labels associated with the Pleasure and Arousal affective categories may introduce a bias, the main objective of this first experiment is to find the words that best represent the meaning of theses affective categories in the target language. Generally, these dimensions are defined using four affective categories: Pleasure and its opposite Misery, and Arousal and its opposite Sleepiness. However, despite the fact that emotional terms are often equated in translation dictionaries, a literal translation may not accurately reflect the meaning of the original emotional term since the terms used to define emotions vary 


\subsection{AN EXPERIMENT-BASED METHOD TO ADAPT AFFECTIVE MODELS TO CULTURE AND LANGUAGE}

in meaning depending on the culture and the language $[23,46]$. To translate these words we have used the following methodology:

- We consulted the translations provided by the four most widely used bilingual dictionaries: The Cambridge [58], Collins [59], Oxford [60] and WordReference [61] English-Spanish Dictionaries. We selected 30 Spanish words for the four affective categories: 8 words for Pleasure, 10 words for Misery, 5 words for Arousal, and 7 words for Sleepiness.

- Fifty people participated in this first experiment: 20 females and 30 males ranging in age between 18 and 60 years old and with different study levels (from secondary school to the doctoral level). The instructions were given to each participant at the beginning of the experiment. Each participant was asked to read each group of words and identify the general affective category represented by the group. Each participant was given an unlimited time to select the word that best represents the concept of the group and to remove those words that clearly do not express the meaning of the affective category of the group.

\section{Experiment 2: Assigning areas to each emotion}

Based on the hypothesis that the Pleasure and Arousal levels of an emotion can vary depending on the culture and language, this second experiment was designed to define the levels corresponding to each emotion in the target language. We decided not to consider other dimensions because recent studies, like [107], have shown that the Pleasure and Arousal dimensions predict the structure in emotion across language families. Moreover, some authors have found that human beings have difficulty and display confusion when they try to assign a Dominance value to the emotions that they are feeling and that this dimension does not show much difference between emotions [107].

One hundred people participated in this second experiment: 40 females and 60 males ranging in age between 18 and 60 years old with different study levels (from secondary school to the doctoral level). To avoid bias, the participants performed the experiment individually and without a time limit. We designed a questionnaire using the four affective categories translated into 


\section{CHAPTER 2. A CULTURALLY ADAPTED REPRESENTATION OF EMOTIONS (SELECTED PAPER)}

Spanish in the first experiment. It was composed of 10 prompts, one for each emotion. At the beginning of the experiment, the instructions were given to each participant individually. For each prompt, the participants were asked to assign a level of Pleasure and a level of Arousal to the 10 selected emotions. The structure of the prompts was designed to collect the Pleasure and Arousal variables individually. To assign these two levels, each prompt was composed of two 7-item Likert scales. The scale for Pleasure was defined from very miserable to very pleased and the scale for Arousal was defined from very sleepy to very aroused. Note that the value 4 corresponds to a neutral value on these scales.

\section{Analysis and Results}

In the first experiment, we define the degree of acceptance of one word $w$ for the affective category $c\left(\operatorname{accept}_{c}(w)\right)$ as the difference between the number of times the word $w$ has been selected as being representative of the affective category $c$ and the number of times the word has been removed. The degree of acceptance for all of the candidate words are represented in Fig. 2.3. For instance, Fig. 2.3a shows that the word Alertado has a degree of acceptance for the Arousal category accept $t_{\text {arousal }}($ alertado $)=25$, while $E x$ citado has accept $t_{\text {arousal }}($ excitado $)=-22$. The negative degree of acceptance means that the participants mostly rejected that word to represent the affective category of the group. The results confirm the hypothesis of the first experiment, and the need to carry out studies of this type when translating affective concepts to other languages and cultures. For example, according to the selected dictionaries, the closest translation into Spanish of the affective concept Misery is Miseria. However, as Fig. 2.3d shows, this word only has an $\operatorname{accept}_{\text {misery }}($ miseria $)=-11$. Therefore, it was not selected to represent this affective category since a great number of people discarded it. For the concept of misery, the participants selected words such as Suffering, Sadness, and Unhappiness (in Spanish Sufrimiento, Tristeza, and Infelicidad). This is probably due to the fact that the concept of misery in Spanish is related more to economic poverty than to suffering. We can also see that the best translation for Arousal is Alerta. For Sleepiness, the words selected 


\subsection{AN EXPERIMENT-BASED METHOD TO ADAPT AFFECTIVE MODELS TO CULTURE AND LANGUAGE}

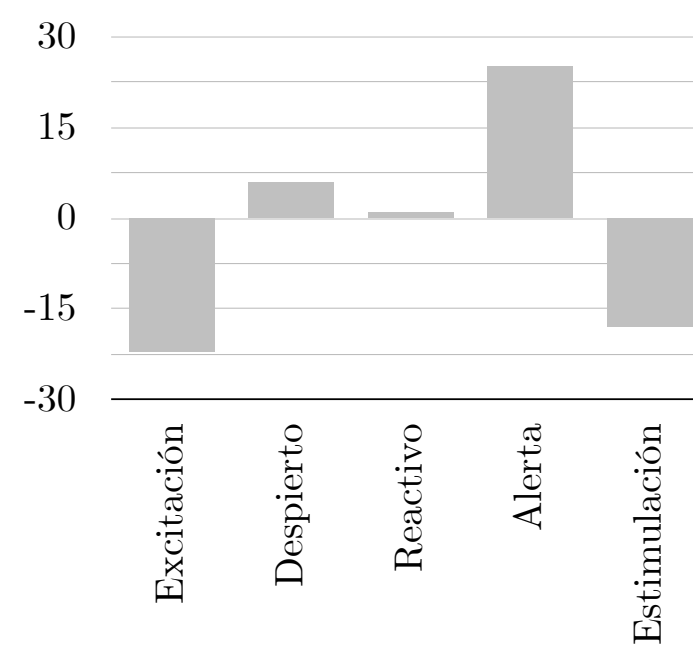

(a) Arousal

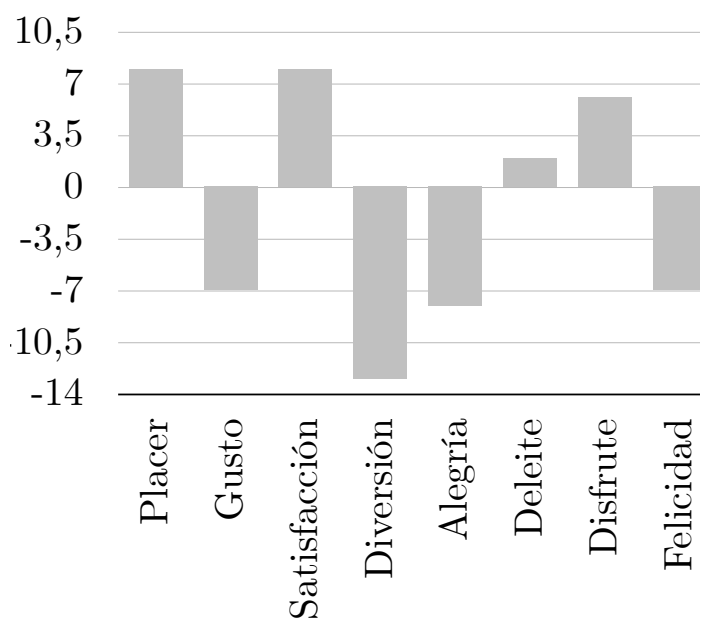

(c) Pleasure

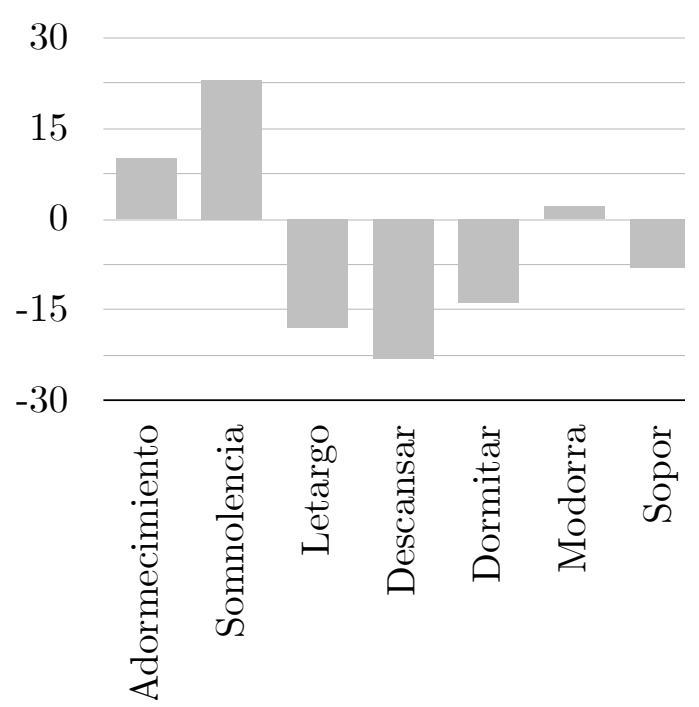

(b) Sleepiness

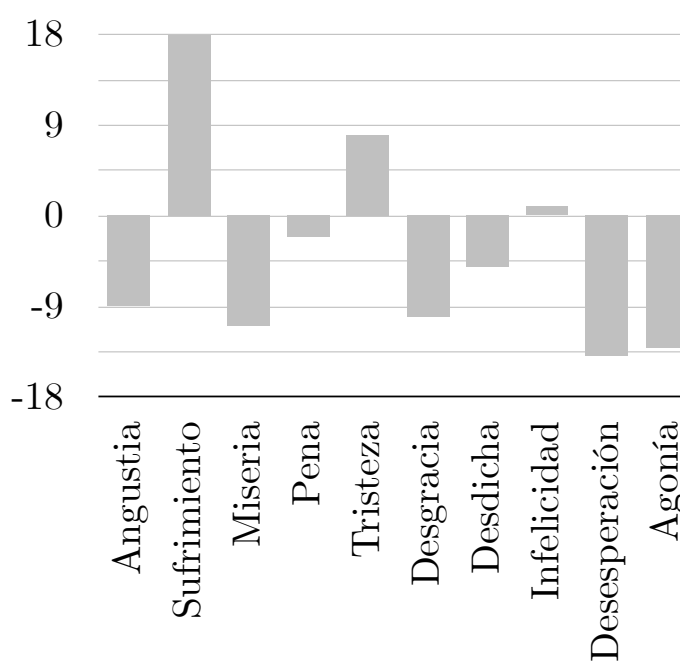

(d) Misery

Figure 2.3: Degree of acceptance of the candidate words for each affective category. 


\section{CHAPTER 2. A CULTURALLY ADAPTED REPRESENTATION OF EMOTIONS (SELECTED PAPER)}

as most representative were Somnolencia and Adormecimiento. Finally, for Pleasure, there is a tie between Placer accept pleasure $\left._{\text {placer }}\right)=8$ and Satisfacción accept $t_{\text {pleasure }}($ satisfaccion $)=8$. The results of the first experiment show that there are some affective categories in which there are more than one word with a positive degree of acceptance. For example, in the case of Pleasure, it is very clear that both Placer and Satisfacción are relevant to the understanding of this abstract concept. Therefore, by selecting one of the two, we could be biasing the translation of Pleasure since each person can have an individual conceptualization that could be influenced by their geographical location, age, or cultural environment. The conclusion we drew from these results was that, in order to avoid a possible bias that could produce misunderstandings, we needed to contextualize each affective category by using more than one word. To determine the appropriate number of words for each affective category, we used the results of the experiment. For the affective categories Arousal, Sleepiness, and Misery, we selected those words with a positive degree of acceptance, obtaining three words for each affective category. However, by applying the same criteria for Pleasure, four possible words were obtained. Therefore, in order to define all of the affective categories homogeneously to avoid misunderstandings, for Pleasure, we decided to select the three words with the highest degree of acceptance.

We used the second experiment to define the levels of Pleasure, Misery, Arousal, and Sleepiness associated to each emotion label. Then, we performed a circular statistical analysis of the results of the second experiment to determine the best circular representation of the selected emotions based on their Pleasure and Arousal components. Henceforth, we will refer only to the dimensions of Pleasure and Arousal, considering that Misery is negative Pleasure and Sleepiness is negative Arousal. First of all, we discarded all of the answers of the participants that represent unexpressed emotion. These emotions are characterized by zero values in the Pleasure and Arousal dimensions, which correspond to the $4^{\text {th }}$ item in the Likert scales. We made this decision taking into account that, with low levels of Pleasure and Arousal, the intensity of the emotions is so low that it can be considered that there is no emotion [183]. Second, for all of the answers of each participant for each selected emotion, we calculated the angle $\alpha$ between the Pleasure axis and 


\subsection{AN EXPERIMENT-BASED METHOD TO ADAPT AFFECTIVE MODELS TO CULTURE AND LANGUAGE}

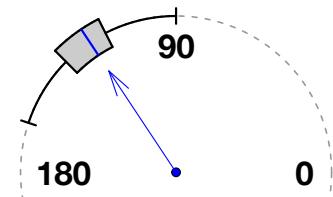

270

(a) Fear

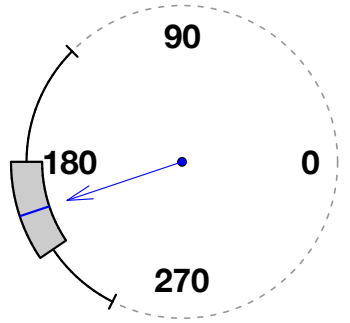

(d) Sadness

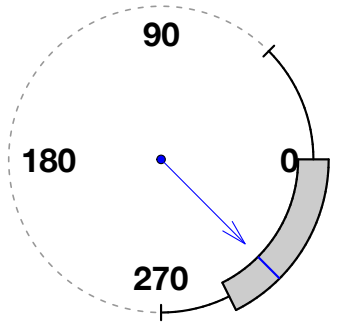

(g) Calm

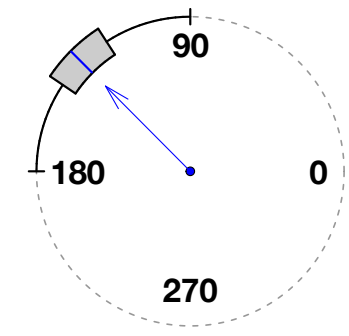

(b) Anger

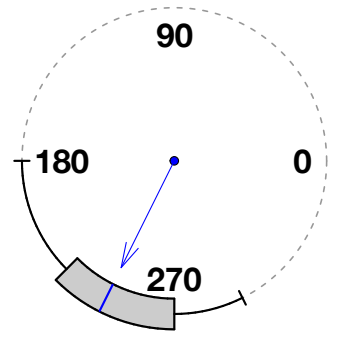

(e) Boredom

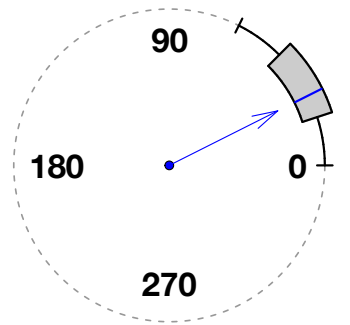

(h) Happiness

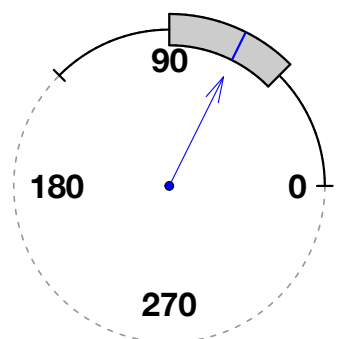

(j) Surprise

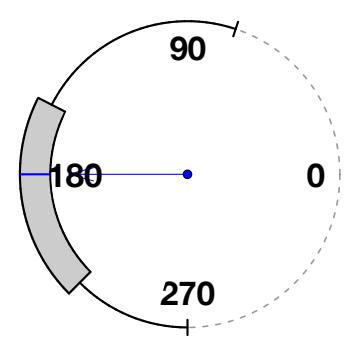

(c) Disgust

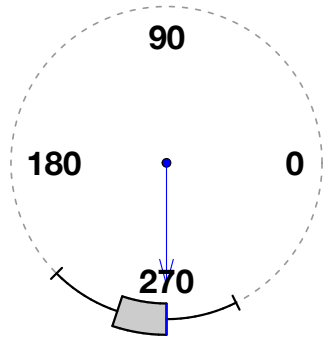

(f) Sleepiness

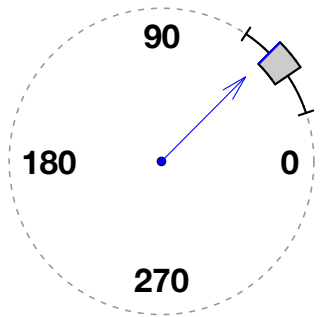

(i) Excitement

Figure 2.4: Circular boxplot graphic for the ten selected emotions in Spanish (see Table 2.1) where the horizontal axis represents Pleasure and the vertical axis represents Arousal. 


\section{CHAPTER 2. A CULTURALLY ADAPTED REPRESENTATION OF EMOTIONS (SELECTED PAPER)}

Table 2.1: Results of the second experiment. $\bar{\alpha}_{e}$ and $\sigma_{e}$ expressed in radians.

\begin{tabular}{lcccc}
\hline Emotion $e$ in & Mean & Mean & Mean & Sd \\
Spanish (English) & $\bar{P}_{e}$ & $\bar{A}_{e}$ & $\bar{\alpha}_{e}$ & $\sigma_{e}$ \\
\hline Felicidad (Happiness) & 0.90 & 0.42 & 0.44 & 0.33 \\
Emoción (Excitement) & 0.76 & 0.64 & 0.70 & 0.18 \\
Miedo (Fear) & -0.58 & 0.81 & 2.19 & 0.27 \\
Enfado (Anger) & -0.74 & 0.66 & 2.42 & 0.29 \\
Tristeza (Sadness) & -0.96 & -0.27 & 3.42 & 0.39 \\
Aburrimiento (Boredom) & -0.41 & -0.91 & 4.28 & 0.38 \\
Somnolencia (Sleepiness) & -0.11 & -0.99 & 4.60 & 0.27 \\
Calma (Calm) & 0.74 & -0.67 & 5.55 & 0.63 \\
Asco (Disgust) & -0.99 & -0.04 & 3.19 & 0.76 \\
Sorpresa (Surprise) & 0.31 & 0.95 & 1.25 & 0.46 \\
\hline
\end{tabular}

the line defined by the (Pleasure, Arousal) point and the origin of the coordinate system. This angle $\alpha$ represents the "meaning" that each participant associates to each emotion in this two-dimensional space. Third, we carried out a circular statistical analysis to eliminate possible outliers. To do this, we estimated the circular dispersion of the data associated to each emotion by the participants.

Table 2.1 summarizes the results of the second experiment: the mean values associated with the Pleasure and Arousal variables for the emotion $e\left(\bar{P}_{e}\right.$ and $\left.\bar{A}_{e}\right)$, and the mean angle $\bar{\alpha}_{e}$ and circular standard deviation $\sigma_{e}$ calculated using the Formula 2.1. To analyze the distribution of emotions in this polar representation, we use circular boxplots [36]. The circular boxplots in Fig. 2.4 show the circular distribution in the Pleasure-Arousal space that we obtained for the 10 emotions. As can be observed, Sadness is related to low levels of Pleasure and mean levels of Arousal (Fig. 2.4d), while Excitement is related to high levels of Pleasure and Arousal (Fig. 2.4i). These graphs also show the great differences between the dispersion levels of the emotions. For example, the dispersion of the Excitement emotion (Fig. 2.4i) is clearly lower than that of the Calm emotion (Fig. 2.4g). This confirms that, by representing the emotion with a simple point, as proposed in previous approaches, it is 


\subsection{AN EXPERIMENT-BASED METHOD TO ADAPT AFFECTIVE MODELS TO CULTURE AND LANGUAGE}

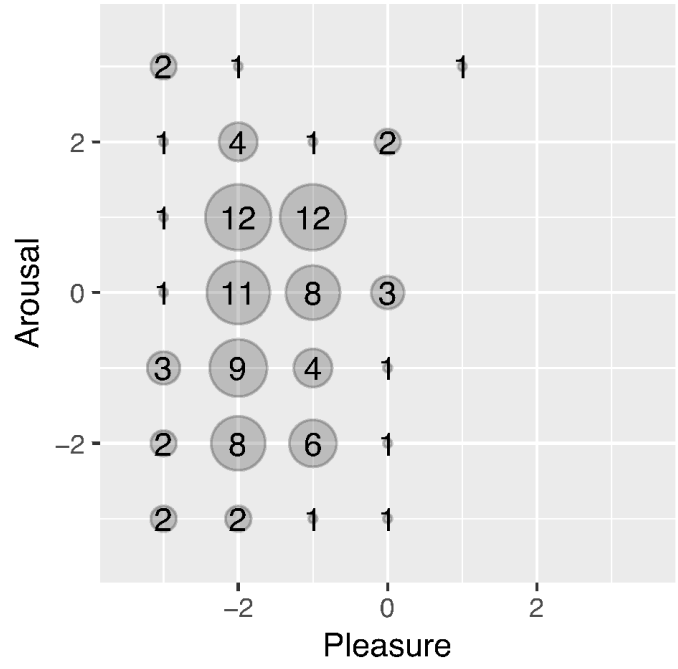

Figure 2.5: Dispersion of the Disgust emotion.

\begin{tabular}{|c|c|c|c|c|}
\hline $\begin{array}{l}-\bullet-\text { Happiness } \\
-x-\text { Excitement }\end{array}$ & $\begin{array}{l}-\triangleleft-\text { Fear } \\
-\Delta-\text { Surprise }\end{array}$ & $\begin{array}{l}\text { - - - Disgust } \\
\text { - *-Anger }\end{array}$ & $\begin{array}{l}-\square-\text { Boredom } \\
-\nabla-\text { Sadness }\end{array}$ & $\begin{array}{l}-+- \text { Calm } \\
-- \text { Sleepiness }\end{array}$ \\
\hline
\end{tabular}

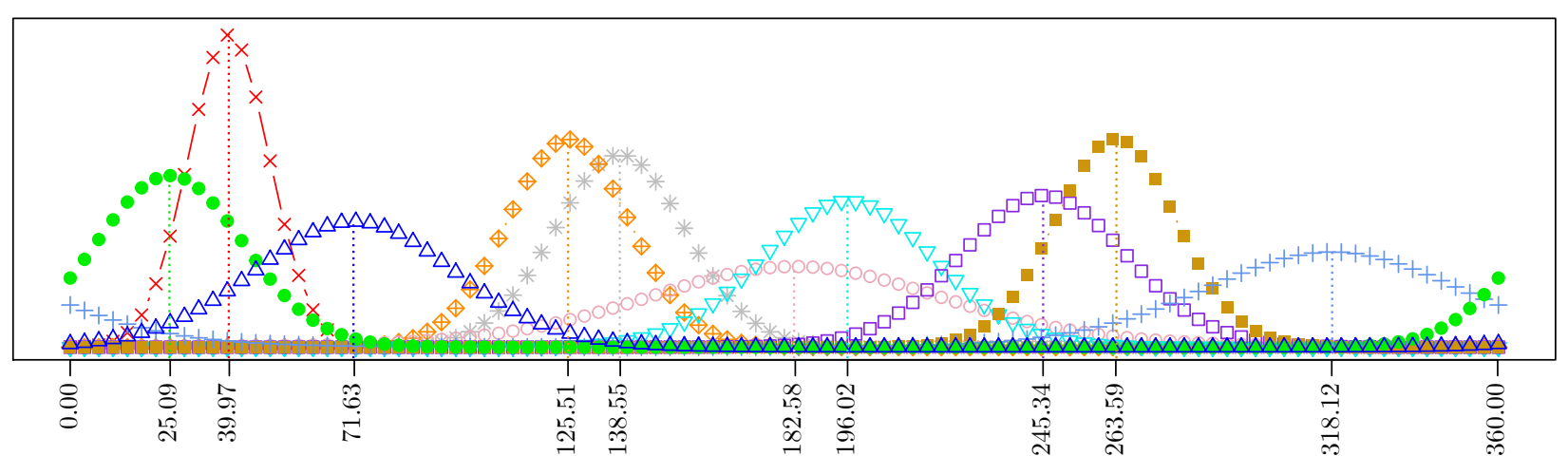

Figure 2.6: Von Mises curves of the ten Spanish emotions (see Table 2.1). The axis represents the angles $\alpha_{e}$ in degrees. Note that the order of the means of the emotions on the plot corresponds to the order of the legend.

performed a simplification that does not correspond to the area that humans assign to emotions in their minds. It also confirms the hypothesis of the second experiment, and the need to perform these types of experiments when modeling emotions in different cultural contexts.

When analyzing the results obtained for Disgust (Fig. 2.4c), we found that it occupies a large part of the dimensional space. The most likely explanation 


\section{CHAPTER 2. A CULTURALLY ADAPTED REPRESENTATION OF EMOTIONS (SELECTED PAPER)}

for this behavior is that in Spanish, the Disgust emotion does not seem to have a clear definition in the dimension of Arousal even though it is clear that the Pleasure value is negative. This behavior is better observed in Fig. 2.5, where the dispersion of the Arousal values for the Disgust emotion is very high (but always with a negative Pleasure value).

From these results, we estimated the circular normal distribution of each emotion. Fig. 2.6 shows the overlap of the von Mises models obtained for each emotion. As can be observed, there are some emotions that overlap each other. For example, the Fear and Anger emotions have similar mean angles $\left(\bar{\alpha}_{\text {fear }}=\right.$ 2.19 and $\bar{\alpha}_{\text {anger }}=2.42$ ), and the same relation can be observed for Boredom and Sleepiness, or Excitement and Happiness. In addition, as expected, it can be observed that the Disgust emotion has a very large standard deviation and is overlapped by several emotions. This representation allows us to estimate the probability of selecting an emotion according to the angle $\alpha$ between the Pleasure axis and the line defined by the (Pleasure, Arousal) point and the origin of the coordinate system. Therefore, instead of defining emotions as areas, we define regions of probability in which emotions can occur with a certain degree of uncertainty. By introducing this uncertainty, the model becomes more versatile, allowing the same point in space to belong to more than one emotion with different probabilities. In addition, with this model, we avoid one of the main problems of a point-based representation because we cannot categorically affirm that a point belongs to one and only one emotion.

We have performed a similar circular statistical analysis of the results obtained by Russell with English speakers [193] (using Formulas 2.1 and 2.2). To demonstrate the need to adapt the emotional models to the language in which it will be used, we have compared these results with those obtained in our experiment with Spanish speakers. In order to determine if the samples are different, we performed a t-Student statistical analysis starting from the null hypothesis $H_{0}$ : the samples are equal. The results are shown in Table 2.2. As can be observed, the p-value for the emotions Happiness, Excitement, Fear, Anger, Sadness, and Sleepiness is less than 0.05. Therefore, we can reject the null hypothesis $H_{0}$ and accept that the samples are significantly different. However, for the Boredom and Calm emotions, the p-value is greater than 0.05. Therefore, the samples are not significantly different. Fig. 2.7a shows the 


\subsection{AN EXPERIMENT-BASED METHOD TO ADAPT AFFECTIVE MODELS TO CULTURE AND LANGUAGE}

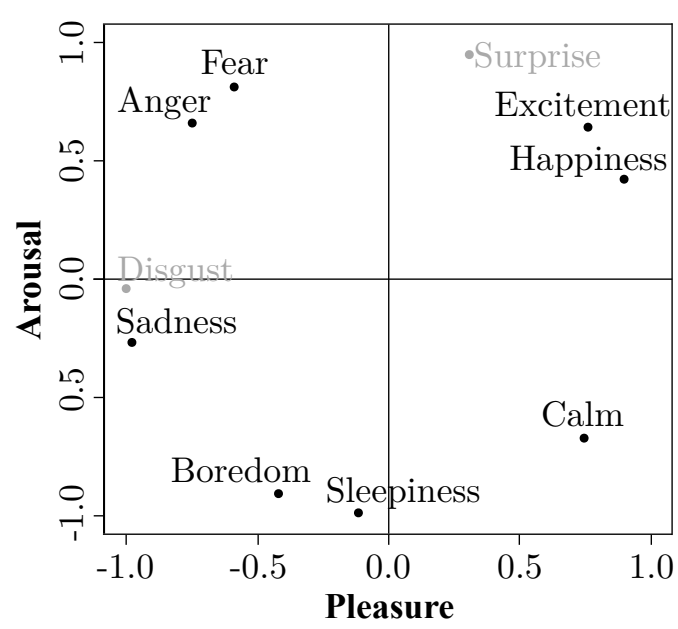

(a) Results of our experiment.

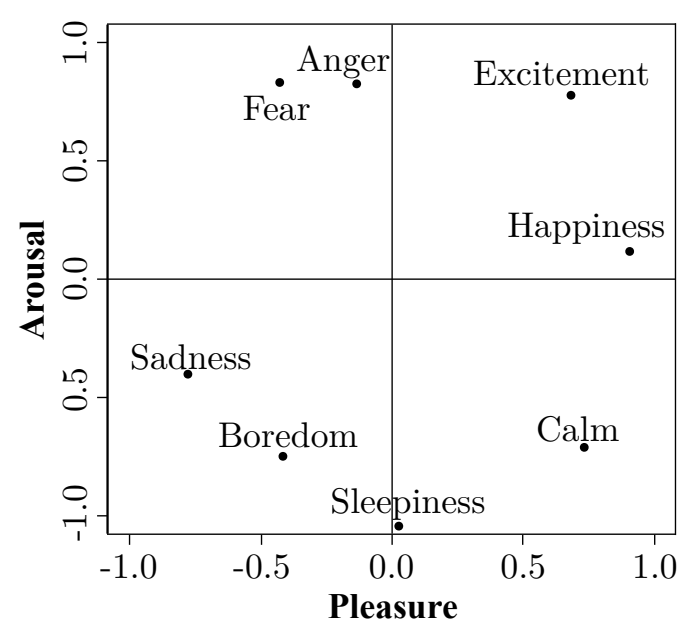

(b) Results obtained by Russell.

Figure 2.7: Results obtained in our experiment (with emotions expressed in Spanish (see Table 2.1)) and Russell's (with emotions expressed in English) [193]. Note that the emotions not represented in the Russell's experiment are shown in gray.

mean position of the 10 selected emotions in Spanish according to their level of Pleasure and Arousal. When we compare the results of our second experiment with those obtained by Russell (Fig. 2.7b), some differences can be easily detected. For example, our experiment shows that the Happiness emotion is related to higher levels of Arousal for European Spanish-speakers than the levels obtained by Russell for British English-speakers. These differences can also be seen in other emotions such as Anger, which is more related to lower levels of Pleasure in Spanish than in English.

We can conclude that, for six of the eight selected emotions, the differences between languages are significant. Therefore, these results confirm the need for experiments of this type when working with emotions in different cultures and languages since there are significant differences in the affective meaning of the words that are used to define emotions. 


\section{CHAPTER 2. A CULTURALLY ADAPTED REPRESENTATION OF EMOTIONS (SELECTED PAPER)}

Table 2.2: Distribution of emotions in European Spanish and British English. (Data for British English obtained from [193]). * indicates $p$-value $<0.05$.

\begin{tabular}{lrrrrrrrrr}
\hline \multicolumn{2}{c}{ Emotions in Spanish } & & & \multicolumn{3}{c}{ Emotions in English } & & \multicolumn{2}{c}{ Statistical comparison } \\
\cline { 1 - 2 } Label/word & Mean $\bar{\alpha}_{e}$ & Sd $\sigma_{e}$ & & Label/word & Mean $\bar{\alpha}_{e}$ & Sd $\sigma_{e}$ & & t-value & p-value \\
\hline Felicidad & 0.44 & 0.33 & & Happiness & 0.14 & 0.58 & & 3.78 & $0.0002^{*}$ \\
Emoción & 0.70 & 0.18 & & Excitement & 0.84 & 0.34 & & -3.27 & $0.0013^{*}$ \\
Miedo & 2.19 & 0.27 & & Fear & 1.27 & 0.58 & & 12.54 & $0.0000^{*}$ \\
Enfado & 2.42 & 0.30 & & Anger & 1.73 & 0.76 & & 7.63 & $0.0000^{*}$ \\
Tristeza & 3.42 & 0.40 & & Sadness & 3.62 & 0.77 & & -1.98 & $0.0488^{*}$ \\
Aburrimiento & 4.28 & 0.39 & & Boredom & 4.20 & 0.69 & & 0.91 & 0.3638 \\
Somnolencia & 4.60 & 0.27 & & Sleepiness & 4.75 & 0.33 & & -2.60 & $0.0102^{*}$ \\
Calma & 5.55 & 0.63 & & Calm & 5.52 & 0.34 & & 0.30 & 0.7612 \\
\hline
\end{tabular}

\subsection{A fuzzy culturally adapted model of affect}

We have defined a fuzzy model to represent emotions in the Pleasure-Arousal space using the results of the second experiment. Our model uses a circular representation in which an emotion is represented by a vector that is defined by a pair of values of Pleasure and Arousal. This vector represents both the type and intensity of the emotion. Taking into account the relationship between the variables of Pleasure and Arousal and the intensity of emotions proposed by Reisenzein [183], we have divided the Pleasure-Arousal space into four fuzzy values of intensity: Strong, Medium, Weak, and Neutral. We have introduced the concept of the Neutral intensity to represent those emotions whose intensity is too low to be considered as elicited.

Therefore, given a point in space defined by its Pleasure and Arousal value, we obtain the intensity of the emotion represented by that point using the modulus of the vector that forms the point with the origin of the coordinate system. Then, we transform this point into a fuzzy value following the model of intensity represented in Fig. 2.8, where the direction of the vector indicates the emotion type. We have used the results obtained in the second experiment to define the areas where the 10 basic emotions have the greatest probability of being located. Fig. 2.8 shows the representation of these probability areas 


\subsection{A FUZZY CULTURALLY ADAPTED MODEL OF AFFECT}

in the circular two-dimensional space. This figure has been obtained from the von Mises model shown in Fig. 2.6 using a single standard deviation. The outer arcs in Fig. 2.8 represent the regions assigned to each emotion. This figure clearly represents the distribution of the basic emotions in the Pleasure-Arousal space for Spanish-speakers.

As mentioned in Section 2.3, when we described the model obtained from the results of the second experiment, the distribution of the basic emotions in the Pleasure-Arousal space produces overlaps and intersections between emotions. This is easily observed in Fig. 2.8: The Excitement emotion intersects with the Happiness and Surprise emotions. Similarly, the Boredom emotion intersects with the Sleepiness and Disgust emotions. The Anger, Fear, and Disgust emotions have also an intersection. Finally, there is an overlap between the Sadness and Disgust emotions. This overlap is largely due to the fact that the Disgust emotion has a high dispersion. The resulting model clearly shows the relationship between emotions and the angle of the vector that we use to identify the emotion.

Next section presents our model which consists of two processes to represent and express emotions: the Emotion Representation Process, a defuzzification process which represents fuzzy emotions in the Pleasure-Arousal space; and the Emotion Expression Process, a fuzzification process which expresses emotions using fuzzy terms in the same way as human beings express the emotion in their own language.

\section{The Emotion Representation Process}

The Emotion Representation Process is a defuzzification process, in which the emotions recognized or elicited by an affective agent are internally represented in the Pleasure-Arousal two-dimensional space. Human beings express emotions using a fuzzy terminology. Therefore, the model must be able to represent fuzzy emotion values in the Pleasure-Arousal continuous space. A fuzzy emotion that is recognized by the agent is internally represented using the mean values of Pleasure and Arousal obtained in the second experiment. In the same way, the intensity is represented in this multidimensional space by using the mean value of each intensity value from the model represented 


\section{CHAPTER 2. A CULTURALLY ADAPTED REPRESENTATION OF EMOTIONS (SELECTED PAPER)}

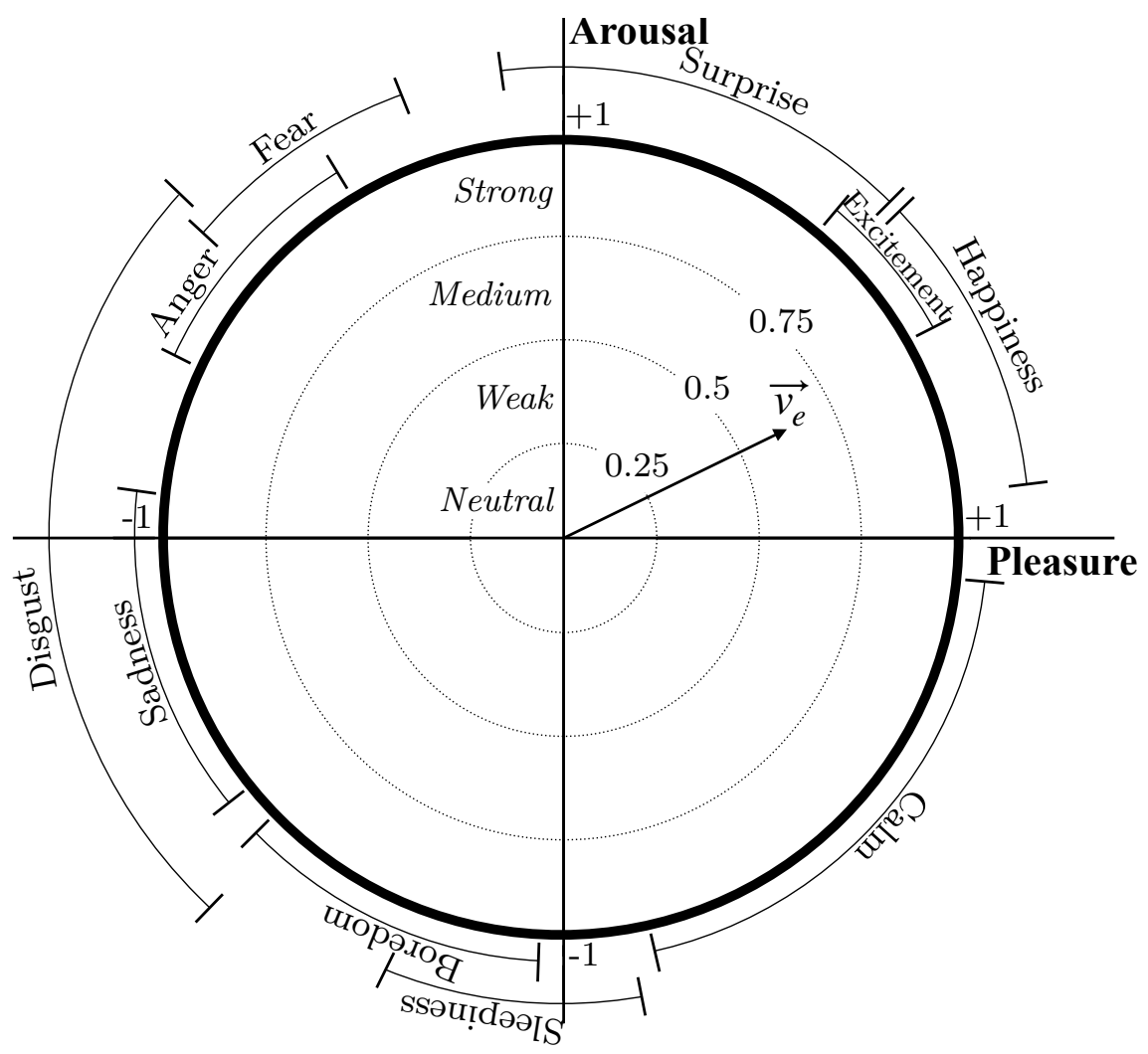

Figure 2.8: Levels of Pleasure and Arousal associated to Spanish emotions (see Table 2.1) using one standard deviation.

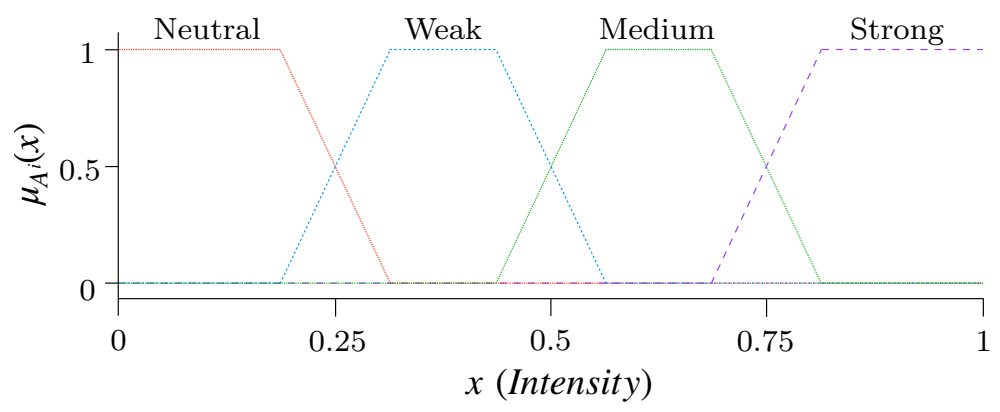

(a) The proposed fuzzy model for the intensity of the emotions in the Pleasure-Arousal space. See Table 2.3 for the linguistic values in Spanish.

Figure 2.9: Fuzzy model for the intensity. 


\subsection{A FUZZY CULTURALLY ADAPTED MODEL OF AFFECT}

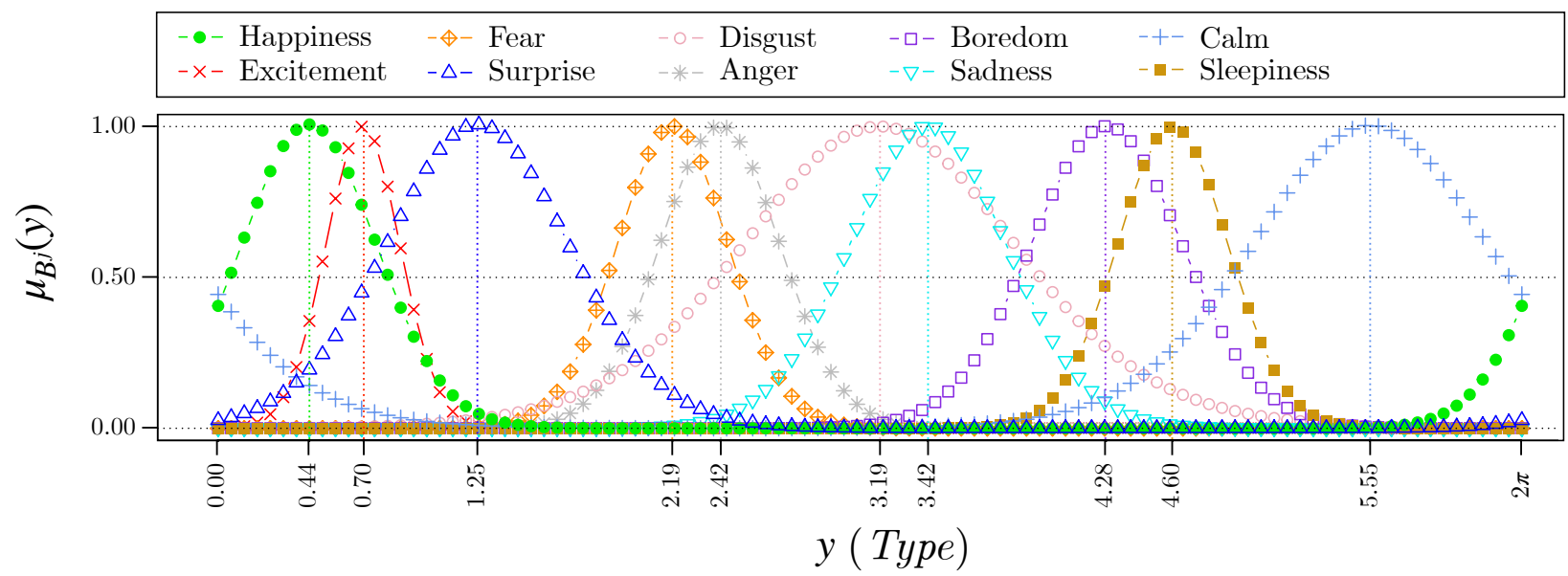

Figure 2.10: Proposed fuzzy model for the type of the Spanish emotions (see Table 2.1) in the Pleasure-Arousal space. Note that the order of the means of the emotions on the plot corresponds to the order of the legend.

in Fig. 2.8. Let us illustrate this process with a simple example. If the emotion perceived by the emotion recognition process of an affective agent is Happiness with a Medium intensity, the agent will internally represent this emotion using a vector $\overrightarrow{v_{e}}$ with a direction $\alpha_{e}=0.44$ radians $(\approx 25.2$ degrees) (see Table 2.1) and a modulus $\left|\vec{v}_{e}\right|=0.625$, which correspond to the Medium intensity (the midpoint between 0.5 and 0.75 according to Fig. 2.8). From this vector $\vec{v}_{e}$ representing the emotion $e$, the agent will obtain the Pleasure $P_{e}$ and Arousal $A_{e}$ values using the following equation:

$$
\left(P_{e}, A_{e}\right)=\left(\left|\vec{v}_{e}\right| \cdot \cos \alpha_{e},\left|\vec{v}_{e}\right| \cdot \sin \alpha_{e}\right)
$$

Therefore, by using this formula, the values of Pleasure and Arousal associated with the "Happiness emotion with Medium intensity" in the Spanish language are 0.57 and 0.27 , respectively. An example of the resulting representation of the vector $\vec{v}_{e}$ in our model is shown in Fig. 2.8.

Note that this representation of the emotion is based on the values of Pleasure and Arousal. Therefore, this representation has a universal semantics [107]. The agent uses this representation of the emotion for all its affective processes. When the agent needs to express an emotion, which is internally 


\section{CHAPTER 2. A CULTURALLY ADAPTED REPRESENTATION OF EMOTIONS (SELECTED PAPER)}

Table 2.3: Parameter ranges for the intensity membership function.

\begin{tabular}{lrrrr}
\hline Intensity level & \multicolumn{4}{c}{ Parameters } \\
\cline { 2 - 5 } in Spanish (English) & $\mathrm{a}$ & $\mathrm{b}$ & $\mathrm{c}$ & $\mathrm{d}$ \\
\hline Neutral (Neutral) & 0 & 0 & 0.2 & 0.3 \\
Bajo (Weak) & 0.2 & 0.3 & 0.45 & 0.55 \\
Medio (Medium) & 0.45 & 0.55 & 0.7 & 0.8 \\
Alto (Strong) & 0.7 & 0.8 & 1 & 1 \\
\hline
\end{tabular}

Table 2.4: $\mu_{A^{i}}(u)$ values for the example $(u=0.625)$.

\begin{tabular}{ccccc}
\hline & Neutral & Weak & Medium & Strong \\
\hline$\mu_{A^{i}}(0.625)$ & 0 & 0 & 1 & 0 \\
\hline
\end{tabular}

Table 2.5: $\mu_{B^{j}}(t)$ values for the example $(t=0.44)$. For the Spanish labels see Table 2.1.

\begin{tabular}{cccccccccc}
\hline & Happiness & Surprise & Fear & Anger & Disgust & Sadness & Boredom & Sleepiness & Calm \\
\hline$\mu_{B^{j}}(0.44)$ & 1 & 0.35 & 0.21 & $\approx 0$ & $\approx 0$ & $\approx 0$ & $\approx 0$ & $\approx 0$ & $\approx 0$ \\
\hline
\end{tabular}

represented in the Pleasure-Arousal space, the agent will use the process presented in the next section.

\section{The Emotion Expression Process}

Any emotion that is internally represented by a vector $\vec{v}_{e}$ is transformed into a fuzzy expression (emotion label and fuzzy intensity) by the Emotion Expression Process. This process allows the agent to express its emotions using a fuzzy expression adapted to the cultural environment in which the agent is located. In this process, a fuzzification of the vector $\overrightarrow{v_{e}}$ is performed transforming the intensity and the type of the emotion into fuzzy values. This process is composed of two fuzzification subprocesses: One for the intensity and the other for the emotion type.

We define the linguistic variable $x$ for the fuzzification process of the in- 
tensity of the emotion as a quintuplet [239]:

$$
x=\left\langle L_{x}, T\left(L_{x}\right), U_{x}, G_{x}, M_{x}\right\rangle
$$

where $L_{x}$ represents the name of the linguistic variable $L_{x}=$ intensity, and $T\left(L_{x}\right)$ represents the set of fuzzy terms (linguistic values for the intensity) that the variable $x$ can take:

$$
T(\text { intensity })=\{\text { Strong, Medium, Weak, Neutral }\}
$$

$U_{x}$ (universe of discourse) represents the range of crisp values that the variable can take in the range $U_{x}=[0,1], G_{x}$ is the syntactic rule that generates the terms in $T$ (intensity), and $M_{x}$ is a semantic rule that associate each linguistic term in $T$ (intensity) with its meaning.

We define a Type- 1 fuzzy logic set $\tilde{A} \in M_{x}$ using a trapezoidal membership function defined by the equation $[126,11]$ :

$$
\mu_{A^{i}}(u)=\max \left(\min \left(\frac{u-a}{b-a}, 1, \frac{d-u}{d-c}\right), 0\right)
$$

where $i \in T$ (intensity), $A^{i}$ represents the $i$ th fuzzy set belonging to $\tilde{A}$, $u$ takes the value of the modulus of the vector of the emotion such that $u \in U_{x}$, and $a, b, c$, and $d$ are the parameters of the membership function (see Fig. 2.9b) that is described in Table 2.3 [11]. Fig. 2.9a shows the resulting fuzzy model.

On the other hand, we define the linguistic variable $y$ for the fuzzification process of the emotion type $\left(L_{y}=\right.$ type $)$ :

$$
\begin{gathered}
T(\text { type })=\{\text { Happiness, Excitement, Surprise, Fear, Anger, } \\
\text { Disgust, Sadness, Boredom, Sleepiness, Calm }\}
\end{gathered}
$$

The universe of discourse is defined in the range $U_{y}=[0,2 \pi]$. We use the results obtained in the second experiment (Fig. 2.6) to define a Type-1 fuzzy logic set $\tilde{B} \in M_{y}$. The proposed membership function for an emotion $t$ is derived from the normalization of the von Mises distribution and is defined as:

$$
\mu_{B^{j}}(t)=\frac{m_{j}(t)-\min _{x \in[0,2 \pi]} m_{j}(x)}{\max _{x \in[0,2 \pi]} m_{j}(x)-\min _{x \in[0,2 \pi]} m_{j}(x)}
$$




\section{CHAPTER 2. A CULTURALLY ADAPTED REPRESENTATION OF EMOTIONS (SELECTED PAPER)}

where $j \in T$ (type), $B^{j}$ represents the $j$ th fuzzy set belonging to $\tilde{B}, t$ takes the value of the angle of the vector of the emotion expressed in radians such that $t \in U_{y}$, and $m_{j}(\theta)$ represents the $j$ th von Mises function defined as:

$$
m_{j}(\theta)=\frac{\exp \left(\kappa_{j} \cos \left(\theta-\overline{\alpha_{j}}\right)\right)}{2 \pi I_{0}\left(\kappa_{j}\right)}
$$

where $\overline{\alpha_{j}}$ is the mean for the $j$ th fuzzy set $B^{j}$ (see Table 2.1 ), $\kappa_{j}$ represents the $j$ th concentration parameter of the von Mises distribution estimated as the inverse of the variance $\left(1 / \sigma_{j}\right)$ [102], and $I_{0}\left(\kappa_{j}\right)$ is the modified Bessel function of order zero used to normalize the function [92]. Fig. 2.10 shows the resulting fuzzy model.

As a result of this process, an affective agent can express an emotion, which is internally represented in the Pleasure-Arousal space by a vector $\overrightarrow{v_{e}}$, by using the same fuzzy expression (emotion label and intensity) that humans use in their language.

Let us again consider our example of an affective agent that has an emotion represented internally by the vector $\vec{v}_{e}$ (Fig. 2.8). The Pleasure and Arousal coordinates of vector $\vec{v}_{e}$ are 0.57 and 0.27 , respectively. Therefore, the direction of the vector is 0.44 radians and the modulus is 0.625 . When the affective agent needs to express this emotion in a specific language, it uses the fuzzification process describe above. This fuzzification process for the intensity produces the results shown in Table 2.4. Therefore, the selected fuzzy value for the intensity is Medium. On the other hand, the fuzzification process for the emotion type produces the results of Table 2.5. If the agent wants to express only one emotion, the agent will select the emotion term/label with the maximum $\mu_{B^{j}}$, which in our example is Happiness. Therefore, the Emotion Expression Process will generate the fuzzy emotion Happiness with Medium intensity as a result of the fuzzification of the emotion represented in the multidimensional space by the vector $\vec{v}_{e}$.

This model can be easily adapted to other languages and cultures. Our model allows a multicultural affective agent to represent and express an emotion using the same interpretation of the fuzzy emotional terms used in the language and culture of its human interlocutor. This can be achieved by 


\subsection{CONCLUSION AND FUTURE WORK}

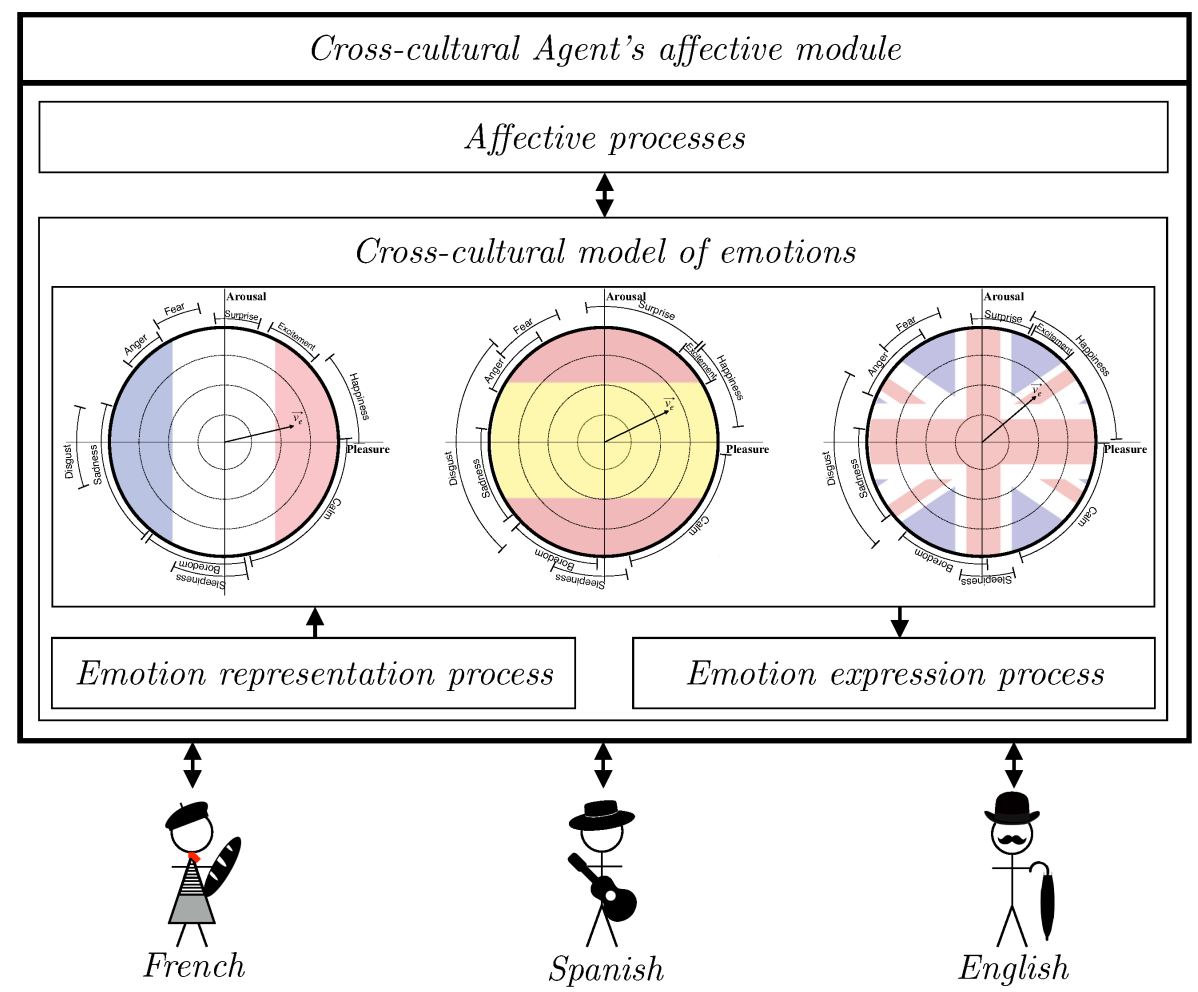

Figure 2.11: Example of a multicultural affective agent module.

defining a specific dimensional Pleasure-Arousal space for each cultural environment in which the agent is located. All these two-dimensional spaces can be easily incorporated to the affective component of the multicultural affective agent (Fig. 2.11).

\subsection{Conclusion and future work}

In this paper, we have proposed an experiment-based method to adapt the emotion representation to the culture and the language in which the emotion will be used. The results of the first experiment have corroborated the importance of adapting the translation of the emotional models taking into account the different meanings that humans give to emotion labels in different cultures. As observed in the translation of the Misery emotion, the literal translation does not have the same contextual meaning for European 
Spanish-speakers and British English-speakers. The results of the second experiment have allowed us to assign different areas in the Pleasure-Arousal space to the different basic emotions. When comparing the results obtained in our experiment with those obtained for British English-speakers, it can be observed that the emotion labels have different values for Pleasure and Arousal depending on the language. This confirms the need for performing this adaptation with experiments when emotional models are used in other cultures and languages.

From the results obtained in the experiments, we have defined a fuzzy model that represents emotions using a Pleasure-Arousal continuous space instead of simple labels. With this cross-cultural dimensional representation, an emotion can be defined as a vector in which the modulus represents the intensity of the emotion, while the type of emotion depends on the direction of this vector. Our model improves the typical representation of emotions based on labels because it allows emotions and their intensity to be easily represented and modified using a dimensional representation.

We have also defined a fuzzification process that determines an emotion label in a specific language from a pair of Pleasure and Arousal values. Thus, using our model, an agent can express emotions using the same fuzzy terminology as humans.

We are currently incorporating this model into the affective agent architecture $G e n I A^{3}$ to improve the simulation of the different affective processes. In addition, we want to analyze whether this emotion representation can be improved by introducing a third dimension (Dominance) to better identify emotions such as Fear or Anger and to analyze the influence of this dimension on social behavior [124]. 



\title{
Chapter 3
}

\section{Computational affective knowledge representation for agents located in a multicultural environment}

\begin{abstract}
Emotions are present in all human interactions. However, there are differences between cultures in how emotions are expressed in interaction. Consequently, when interactions are translated into another language, this culturally relevant information can be lost. In this paper, we show the results of two experiments conducted to adapt a new model of affect representation, based on a polar coordinate system in a space composed of pleasure and arousal dimensions, to the Portuguese and Swedish languages. In the first experiment, we determine the most appropriate labels to define the pleasure and arousal dimensions in each language. In the second experiment, we identify the most suitable values of pleasure and arousal dimensions for each emotion in Portuguese and Swedish. The results obtained in these experiments are compared with a previous experiment conducted with European Spanish-speaking participants. The analysis of the results shows the importance of adapting the affect representation models to the cultures and languages in which they will be used. We demonstrate significant differences in the values of pleasure and arousal associated with emotions across languages and cultures. The results also show that there are not significant differences in gender or age when associating levels of pleasure and arousal to emotions.
\end{abstract}




\subsection{INTRODUCTION}

\subsection{Introduction}

When analyzing human behavior, it is common to find situations in which emotions prevail over logic, conditioning our capacity for judgment and decision making and altering our behavior. Therefore, considering affective states in software processes can improve the simulation of human behavior, the simulation of human social organizations, and human-computer interaction. In fact, systems that are capable of recognizing and simulating affective states have proved to be more credible and more reliable, thus reducing the frustration and stress of human users and improving human-machine interaction [165].

Human affective behavior encompasses a set of constructs and cognitive processes that make it complex to simulate using software agents. One of the main constraints on simulations comes from the way in which humans interpret affective knowledge. To express, represent, and interpret the affective knowledge, humans typically use different abstractions and metaphors. For example, through the use of words or labels such as "Sadness" or "Joy". This way of representation is commonly used in the area of affective computing [170] to generate models for recognition, interpretation, processing, and simulation of affective states $[17,20,74]$. However, the use of words or labels is a simplification that does not allow to represent all the information inherent to affective states such as the intensity with which an affective state is perceived. An alternative to this representation is the use of models based on different cognitive dimensions. One of the most common dimensional models in literature is the Core Affect model [194]. That model defines affective states through two dimensions: pleasure and arousal. Dimensional models have been widely used in affective computing [6, 81]. Still, existing proposals also simplify affective states, losing some of the information inherent to affective states. Moreover, it should be noted that, being cognitive constructs associated with different words, culture and language condition the representation, expression, and interpretation of affective states. Representing affective states while capturing cross-cultural differences poses a challenge since, the cognitive conceptualization of an affective state, may vary across cultures and languages thus influencing the levels of pleasure and arousal as- 


\section{CHAPTER 3. COMPUTATIONAL AFFECTIVE KNOWLEDGE REPRESENTATION}

sociated with that affective state. The use of representation models that are not adapted to the culture and language may produce misunderstandings and errors that compromise the simulations of affective behavior and users' experience. Therefore, establishing representation models capable of adapting to culture and language is a step towards in improving the simulation of human behavior and the personalization of human-machine interaction $[23,196]$.

In this paper, we present the results of two experiments conducted with Portuguese and Swedish participants to adapt a pleasure-arousal representation space to both cultural environments. Based on the results obtained, we defined the probability models for each affective state label. These probability models can be used to represent recognized affective states in the pleasurearousal space and allow software agents to express their affective states in the same terms used by humans in different cross-cultural environments. Then, we compare the results with the previous study conducted with European Spanish speakers. We finally present a computational model of affect adapted to Portuguese and Swedish and a potential application of this cross-cultural model in the area of human-aware planning.

The rest of this paper is organized as follows. In Section 3.2 we present the most relevant theories and models for the representation of affective states. Section 3.3 presents our model of affect representation. In Section 3.4 we describe the methodology used in the experiments conducted to determine the levels of pleasure and arousal associated with affective states in Portuguese and Swedish. The results obtained in the experiments are shown in Section 3.5. Then, a discussion of the results obtained along with the definition of the cross-cultural model of affect is provided in Section 3.6. Section 3.7 proposes potential applications for our representation model of affect. Finally, the main conclusions and some future works are presented in Section 3.8.

\subsection{Related work}

Affective states are constructs that define different experiences, including emotions and moods. On the one hand, emotions can be understood, in general terms, as immediate responses to a specific stimulus [140]. On the 


\subsection{RELATED WORK}

other hand, moods are described in the literature as affective responses that have a longer duration than emotions, ranging from minutes to days or weeks [90]. In addition, mood is not necessarily related to a specific stimulus [44]. Instead, moods are generally triggered by the achievement of a set of cognitive and affective processes.

From the area of affective computing [170] different approaches focused on the recognition, interpretation, processing, and/or simulation of different emotional behaviors and affective states have been conducted [35, 6, 221]. However, not much attention has been paid to the development of models to represent the knowledge related to affective states specifically designed for its use in computational systems. To represent and express the complexity of knowledge related to affective states, humans usually resort to the use of different metaphors and abstractions. One of the most recurrent classifications in the literature divides these metaphors and abstractions into two approaches: categorical and dimensional [93].

Categorical approaches are based on the use of a limited set of words or labels to define the different affective states [66]. These labels provide a means to verbalize affective states, which is a key factor in human affective interactions. One of the best known models is the model of Basic Emotions proposed by P. Ekman in which six labels are defined: "Sadness", "Happiness", "Anger", "Fear", "Disgust", and "Surprise" [65]. This model is one of the most widely used in the area of affective computing to express, recognize, interpret, and represent affective states [20, 173, 74]. For example, in [17], a model of emotion recognition is presented. The authors propose to use the six basic emotions proposed by Ekman to classify emotions recognized in text. However, by a label based representation we assume a high level of abstraction since we condense the entire spectrum of affective states into a limited set of labels. Therefore, we are losing part of the affective information, such as the intensity of the affective state, or the probability of moving from one affective state to another depending on a certain stimulus [71, 163, 232]. For example, if a person is "Sad" and receives good news, the probability of transitioning to a positive affective state such as "Joy" may be lower than if the person is "Happy". In addition, this representation assumes that labels/words representing emotions have the same meaning in all cultures and languages. 


\section{CHAPTER 3. COMPUTATIONAL AFFECTIVE KNOWLEDGE REPRESENTATION}

Nonetheless, depending on the model of emotional representation being used, translating emotions from one language to another can lead to cultural misunderstandings since emotions are not always understood in the same way in different cultures and languages [196]. In fact, there are some labels that express affective states that are very difficult to translate from one language to another. For example, the German vocabulary has an extensive catalog of affective labels (or words) defining emotions such as "Schadenfreude", which can be summarized as "pleasure from the suffering of others", or "Sehnsucht", which can be described as "a strong desire for an alternative life", but whose overall conceptualization is very difficult to describe by means of a word or label in other languages such as English or Spanish [107, 137].

Theories based on dimensional approaches allow reducing the level of abstraction by representing affective states through different dimensions. These theories have their origin in psychological constructivism, according to which a finite set of labels cannot establish affective states since they depend on the individual's subjective experience that occurs in a particular context (e.g., a particular culture or language) $[151,101]$. One of the most representative dimensional models is the Core Affect model proposed by J.A. Russell [194]. In that model, affective experiences are defined as emotional cues and are represented by two dimensions: pleasure and arousal. More specifically, emotions can be defined as high or low arousal (relative to the emotional arousal dimension) and positive or negative (considering the emotional pleasure dimension). Russell also evidenced through experimentation that the labels used to define the different affective states followed a circular pattern along these two dimensions. From these results he developed the Circumplex Model of Affect [174]. The Circumplex Model of Affect uses the multidimensional scaling of labels or words that define emotions [193], concluding that the emotional pleasure dimension and the emotional arousal dimension are the ones that best support the judgments people make about emotions [192, 68]. In that model, all the labels referring to the different affective states are represented as points composed of pleasure and arousal components and are organized in a circular way around these two dimensions, conceptualizing emotions as being systematically related to each other [223], which is also the idea defended by H. Scholesberg [202]. Being a representation based on continuous 


\subsection{RELATED WORK}

dimensions, it is possible to establish relationships between the distribution of the different affective states. For example, it can be assumed that, in general, affective states related to the word "Joy" are related to positive values of the pleasure and arousal dimensions, while those related to the word "Sadness" are related to negative values on these two dimensions.

Some authors have discussed the number of dimensions that define affect. For example, in [154], it is proposed to introduce the dominance dimension to the pleasure-arousal space resulting in a model known as the PAD model. Dominance dimension allows to disambiguate some emotions that are overlapped when only the dimensions of pleasure and arousal are used. Nevertheless, some studies show that this dimension does not have a substantial impact on the representation of affect across cultures [107] and that humans exhibit difficulties and confusion when they try to assign a dominance value to the emotions that they are feeling [122].

In addition, dimensional models, such as the Circumplex Model of Affect, make it possible to highlight the differences that appear across cultures. Over the years, different authors have shown, through experimentation, the existence of variations in the levels of pleasure and arousal associated with each affective state in different cultures and languages [196, 23, 46]. For example, the study presented in [107], analyzed the discrepancy of 24 emotional labels in about 2500 languages. That study concludes that there are significant variations between the values of pleasure and arousal across cultures. Therefore, the direct translations of emotion labels can be subject to bias because the meaning of the words can differ between languages. The authors also evidenced that the differences become smaller the closer the geographical proximity of the language families is.

The circumplex model of affect has also been used in affective computing $[243,204]$. For example, in [136], a model for estimating the facial expressions of a humanoid robot is presented. Authors use the Circumplex Model of Affect to represent emotions as points composed by the pleasure and arousal dimensions. However, the use of the Circumplex Model of Affect in affective computing also presents some disadvantages. Let us suppose that we define affective states labels by a single point in the dimensional representation space, and then we generate a random point in that space. If the point does 


\section{CHAPTER 3. COMPUTATIONAL AFFECTIVE KNOWLEDGE REPRESENTATION}

not match with any of the points predefined for each affective state label, it is not possible to determine the label associated with that point. A possible approach, used in some proposals $[118,131]$, consist in estimating the distance of a random point from each of the predefined points for the affective labels in the model and then, assign the nearest affective label. By this approach it is necessary to assume that the distribution of affective labels in the pleasure-arousal space is equal and uniform. Although, this way of representing affective labels is artificial and is far from how affective states are distributed throughout the pleasure-arousal space as demonstrated in work presented at [220]. In addition, in a point-based approach, it is complex to assign a level of uncertainty that a random point belongs to a certain label or represent the transitions between two affective states. It is also difficult to simulate other affective behaviors such as the influence on the affective state of other affective factors like personality [216]. Still, in a point-based representation, the intensity of affective states is not considered [34]. Despite, there is empirical evidence that establishes a direct relationship between the levels of pleasure and arousal and the intensity with which different affective states are experienced. This means that, values close to zero in both dimensions would indicate very low or no intensity. In contrast, values close to one would indicate high intensity [183].

A possible alternative to the point-based representation is the vector-based representation. In this representation, the direction of the vector is used to determine the label that represents a point in the pleasure-arousal space. For example, in [111], a vector-based representation using pleasure-arousal space to express emotions in a robot is proposed. In that model, each emotion is represented by a uniform range of fifteen degrees. Note that, the emotion "Glad" is defined in the range from 0 to 14 degrees while "Happy" is defined in the range from 15 to 29 degrees. Therefore, that representation presents the same problem as the point-based representation, since the way in which the variations in the dispersion of affective states are represented is symmetrical and, consequently, far from the mental representation that humans have of affective states.

Finally, there seems to be a trend towards the development of affective models based on fuzzy logic $[67,116,108]$. The fuzzy logic allows adding 
uncertainty to the representation model by allowing a point in the pleasurearousal space to belong to more than one label. Moreover, fuzzy logic allows us to express affective states in the same terms that humans use (e.g., "very sad" or a "little happy"). For example, in [121], a fuzzy logic-based model for translating emotional labels from Spanish to English is proposed. A methodology based on an experiment in which 8 Spanish speakers assigned values of pleasure, arousal and dominance to 30 Spanish emotional labels/words was proposed to establish the discriminant values of the fuzzy membership functions for each emotion. The results were compared with the models obtained in the previous experiment conducted with English-speaking participants presented in [123]. Through this comparison, a set of fuzzy functions to translate the emotional labels between the Spanish and English languages were obtained.

\subsection{A cross-cultural model of emotions for affective computing}

In [220], we present a new cross-cultural model of emotion representation for affective computing. Our model differs from the representations discussed in Section 3.2, by providing a new system for representing affective states based on the use of probability areas to define each affective state in the pleasure-arousal space. By using probability areas we add a certain level of uncertainty, since each point in the space composed of the pleasure and arousal components has a certain probability of belonging to each affective label. Our model follows a vector-based representation in a polar coordinate system in which the direction indicates the probability of experiencing a particular affective state and the modulus indicates its intensity. However, in a polar coordinate system, traditional linear statistical models may not be suitable since they do not satisfy the linearity constraint because they are constrained between 0 and $2 \pi$. The main problem underlying the application of linear statistics arises when studying the probability regions near 0 and $2 \pi$ since, in a linear model, these two regions would be independent but, in a polar coordinate system, these two regions are dependent. An approach 
to address the problem of linearity can be found in [106], where a model to represent emotions using a vector-based representation in the pleasure and arousal dimensions is proposed. In that proposal, the representation space is modified by displacing the coordinates' origin to the negative extreme of the arousal dimension. As a result, the model is bounded between 0 and $\pi$, thus reducing the problem that appeared in a representation based on a complete polar coordinate system. Still, this representation has two disadvantages: on the one hand, there is a decontextualization of the sense of negative arousal, which is related to emotions such as "Sleepiness", "Sadness", "Boredom", or "Calm". On the other hand, this representation modifies both the modulus of the vector and its direction, since the origin of coordinates of the vector is located in the negative component of the arousal dimension. By modifying the modulus of the vector, the relationship between the intensity of the affective states and the values of the pleasure and arousal components is blurred. To avoid these issues we propose to replace the common linear statistics with circular statistics [18]. Circular statistics are designed for data that present a circular distribution, as is the case of affective states across the dimensions of pleasure and arousal. The most suitable approach to estimate the dispersion in a circular representation space composed of the pleasure and arousal dimensions is through the circular standard deviation, which is defined as follows $[169,109]$ :

$$
\sigma=\sqrt{-2 \ln \bar{R}}
$$

where

$$
\bar{R}=\sqrt{\bar{P}^{2}+\bar{A}^{2}}
$$

where $\bar{P}$ and $\bar{A}$ represent the arithmetic mean for the pleasure and arousal dimensions respectively.

On the other hand, to estimate the probability distribution in a circular representation space the von Mises distribution [102] can be considered. This 


\subsection{A CROSS-CULTURAL MODEL OF EMOTIONS FOR AFFECTIVE COMPUTING}

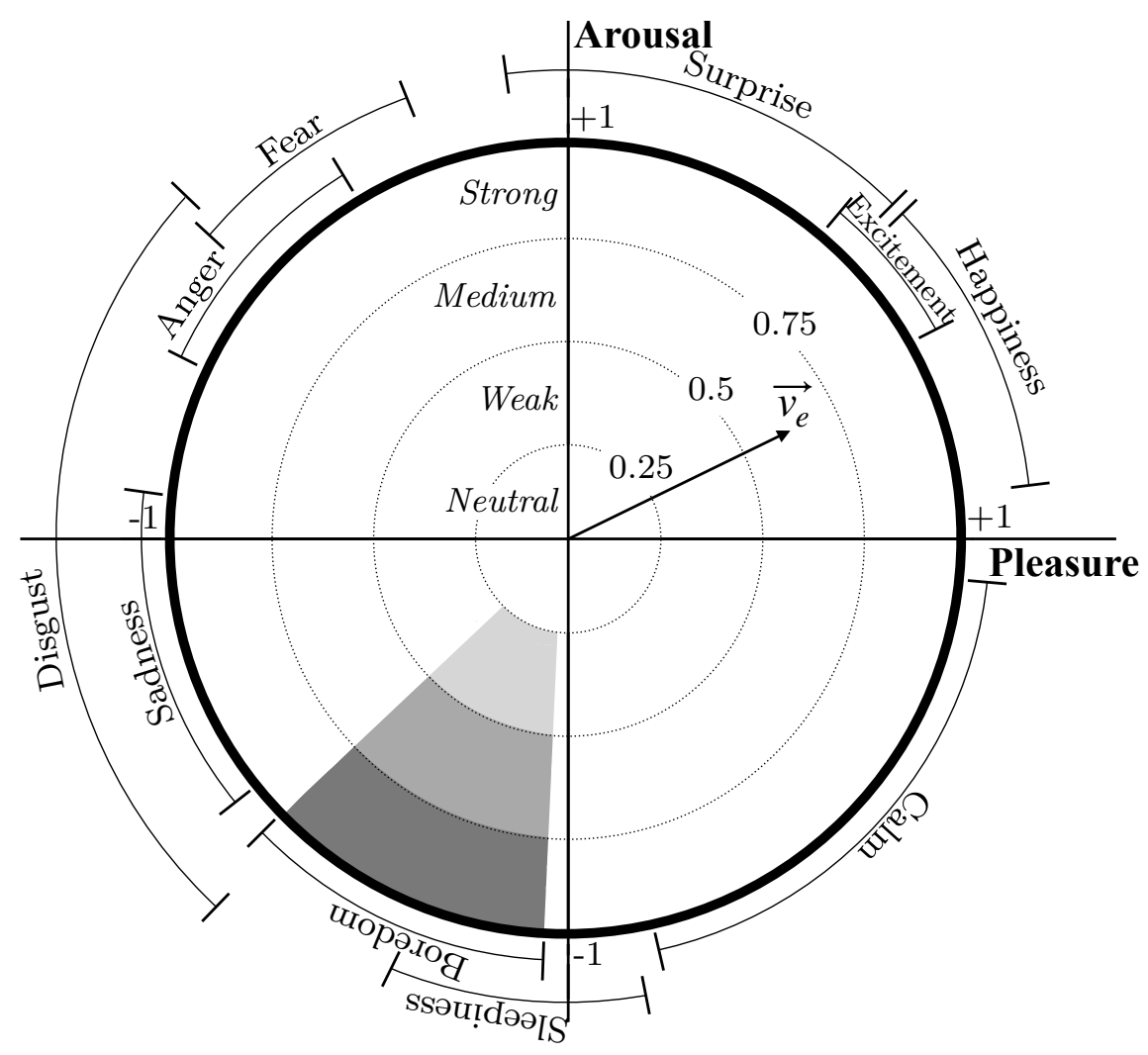

Figure 3.1: Distribution of probability functions in the pleasure-arousal space using one standard deviation for Spanish.

distribution allows estimating the Gaussian (or normal) distribution in circular data. Given an angle $\alpha$, the von Mises probability density function is defined as follows:

$$
f(\alpha \mid \mu, \kappa)=\frac{e^{\kappa \cos (\alpha-\mu)}}{\left.2 \pi I_{0}(\kappa)\right)}
$$

where $\mu$ represents the mean of the distribution, $\kappa$ represents the concentration parameter of the distribution which is calculated as the inverse of the circular variance $\left(1 / \sigma^{2}\right)$, and $I_{0}(\kappa)$ is the modified Bessel function of order zero used to normalize the function [92].

Figure 3.1 shows an example of our model of emotion representation adapted to European Spanish-speakers. In this example, the outer rings show the probability areas of each of the ten emotions using one circular standard deviation, 


\section{CHAPTER 3. COMPUTATIONAL AFFECTIVE KNOWLEDGE REPRESENTATION}

the vector $\vec{V}_{e}$ represents an affective state, and the inner rings represent four regions of intensity: Strong, Medium, Weak, and Neutral. The grey shaded area shows an example of the probability region related to the emotion "Boredom" using one circular standard deviation. Note that the different grey tones represent the different intensity values for the "Boredom" emotion. We can represent the different affective states through this model, abstracting from the labels that represent each affective state, which will only be necessary when a software agent needs to express, interpret, or represent an affective state. Vectors can be modified over time by the occurrence of a sequence of events that happen to an agent. This makes it possible to evaluate the relationship between previous and current affective states, allowing the simulation of different human affective behaviors. For example, if the agent's has an affective state at instant $t_{n}$ and receives a new positive event at instant $t_{n+1}$, we can adapt the agent's emotional response to the affective state it had in $t_{n}$ by modifying the intensity of the emotion generated by the positive event in $t_{n+1}$.

Although the representation of affective states through the dimensions of pleasure and arousal can be considered universal [174, 107], probability areas associated with each affective state depend on the culture and language in which the affective state is needed to be interpreted, expressed, or represented. The direct use of a dimensional model without adapting it to the cultural environment in which it will be used, can produce misunderstandings in the process of selection or representation of the emotion, resulting in erroneous behaviors that will reduce the realism of the simulation of the emotional behavior. Therefore, in our design, we proposed an experiment-based methodology to establish the levels of pleasure and arousal for each emotion in the language in which the model is to be used. Our experiment-based methodology can easily modify these probability areas to include different emotions and adapt the model to the cultural environment in which it is being used.

In addition, through this multidimensional cross-cultural model of affect, an agent can have more than one probability model for different languages and adapt its model of representation and expression of affective states to the language and culture of its human interlocutor. To do that, we establish 


\subsection{EXPERIMENT DESIGN}

two processes based on a fuzzy logic approach: the Emotion Representation Process, which is a defuzzification process, which allows representing words related to affective states in the pleasure-arousal space adapted to the cultural environment; and the Emotion Expression Process, that is a fuzzification process, which determines an emotion label in a specific language from a pair of pleasure and arousal values [220]. These processes allow the agent to express its affective state and represent the perceived affective state using the same interpretation of the fuzzy emotional terms used by its human interlocutor and adapted to his/her cultural environment.

\subsection{Experiment design}

To find the areas associated with each affective state word or label in a target language, we used a methodology based on two experiments: in the first experiment, the words used to represent dimensions of pleasure and arousal are translated into the target language; then, the results of the first experiment are used in the second experiment to obtain the levels of pleasure and arousal associated with each affective state in the target language. In this paper, we present the results of applying this methodology to define computational models for representing affective states in Portuguese and Swedish.

\section{Methodology of the first experiment}

The current study aims to minimize bias in the labelling of emotions, while recognizing prior research results. In the literature there are different references to the pleasure-arousal dimensions. Generally, these dimensions are defined by four affective categories: pleasure and its opposite misery, and arousal and its opposite "Sleepiness" [193]. However, as stated in Section 3.2, the terms used to define affective states or categories are culturally and linguistically dependent. Therefore, using a literal translation of these terms may result in cultural misunderstandings, as the term associated with the translation may not fully reflect the original meaning of an affective category label. This first experiment is designed to identify the labels that best repre- 


\section{CHAPTER 3. COMPUTATIONAL AFFECTIVE KNOWLEDGE REPRESENTATION}

sent the meaning of the pleasure and arousal dimensions in a target language (in this work the target languages are Portuguese and Swedish).

The problem of translating into other languages the terms that humans associate with affective states and affective categories has been explored for years in the literature $[23,46,107]$. For example, a prior study presented in [132], observed interpretational issues concerning the naming of basic dimensions in the previous pleasure and arousal models, and suggests a labelling system for the pleasure-arousal space consisting of forty-eight emotion adjectives in English. A proceeding study by I. Knez and S. Hygge [127] evaluated a Swedish translation of the forty-eight emotion adjectives observed a reasonable consistency with earlier pleasure and arousal models. Similarly, the studies conducted in $[48,143]$ proposed some adjectives to translate these affective categories in specific domains. These adjectives can thus be a good starting point for the present study.

\section{Hypothesis}

The main hypothesis to be validated by this first experiment is that the literal translation of the labels associated with the dimensions of pleasure and arousal may not be adequate across different cultures and languages. The use of a single translated label may not encompass the original global construct.

\section{Participants}

Participants were recruited through Prolific [166]. Prolific is an online platform for conducting experiments that handles the recruitment of participants and the acquisition of participants' personal information such as their nationality, age, and gender. The platform provides different filters to establish the best suited participants for each experiment. When participants successfully complete the experiment Prolific handles the payment. In case participants do not complete the experiment properly (e.g., they fail attention check questions) they suffer a penalty that affects their eligibility for other experiments and they do not receive any economic reward. 


\subsection{EXPERIMENT DESIGN}

In this first experiment, we required participants' nationality, place of residence, and place of birth to be either Portuguese and Swedish in each case. In addition, we also specified that the first language should be Portuguese or Swedish in each case and that the age should be over 18 years old. Finally, we set an approval rate of over $90 \%$. This approval rate measures the percentage of studies successfully completed by the participants and, in Prolific, is used as a measure of the quality of the participants.

For this first experiment, 50 participants were recruited for each of the target languages. In the Portuguese experiment, the sample was divided into 15 women and 35 men ranging in age from 18 to 67 years old $(\mu=26.28$, $\sigma=9.98)$. The mean of the approval rate was situated at 99.36 with a minimum of 95 and a mean of 73.84 studies performed successfully.

For the Swedish experiment, the sample was composed of 13 women, 35 men, and 2 participants who preferred to not indicate their gender. In this case, the age of the participants ranged from 18 to 65 years old $(\mu=29.96, \sigma=$ 9.99). The mean of the approval rate for Swedish participants was situated at 99.50 with a minimum of 95 and a mean of 115.32 studies performed successfully.

\section{Materials}

To translate the four affective categories associated with the dimensions of pleasure and arousal (i.e., pleasure and its opposite misery, and arousal and its opposite "Sleepiness") into the target languages (i.e., Portuguese and Swedish), we followed the methodology presented in [220]. According to this methodology, for the first experiment (concerning identification of suitable pleasure-arousal end-points), a set of labels was chosen for each language. This labels were selected from the common Portuguese and Swedish labels proposed in $[48,143]$ and [127] respectively. With the aim to minimize bias, we complemented both set of labels with different synonyms collected from the dictionaries: Priberam [177] and Infopedia [105] for Portuguese and Bab.la [12] and Synonymer [214] for Swedish. Synonyms were included as found in the dictionaries, while excluding synonyms that did not fit with the arousal and pleasure end-points. Finally, from these research, we select 28 words 
for Portuguese and 30 words for Swedish. Then we divided these words into four sets of words, approximately 5-9 words in each set for Portuguese (see Figure 3.2) and with approximately 7-9 words in each set for Swedish (see Figure 3.3), representing end-points (low/high pleasure and low/high arousal).

To explore the words that participants related most closely to each of the four affective categories in each target language, we designed an online questionnaire that was composed of four sections, one for each affective category. In each section, two checkbox questions were designed to identify the best words to translate each affective category. The first question asked each participant to read a set of words and select the word (or words) that clearly did not express the same meaning as the rest of the words in the set. In the second question, the same set of words was shown and each participant was asked to read the set of words and select the word (or words) that best represented the concept of that set.

We added an extra section containing an attention check question. Following the same structure as the questions in the previous sections, a set of six words was shown, five of which represented color names and one a name of an animal. Each participant was asked to carefully read the set of words and to select the word (or words) that did not represent the same concept as the rest of the set.

\section{Procedure}

In order to register for the questionnaire, participants had to enter their Prolific ID. At the beginning of the experiment, participants were provided with the instructions describing the task to be performed along with two examples for illustrative purposes. Then, participants were asked to complete the four sections of the questionnaire without a time limit.

\section{Methodology of the second experiment}

In the second experiment (concerning definition of emotions in terms of pleasurearousal), the aim was to detect the regions of the pleasure-arousal space associated to each emotion label in Portuguese and Swedish. Initially, for this 


\subsection{EXPERIMENT DESIGN}

experiment we selected the ten emotions proposed in [196]: "Fear", "Surprise", "Disgust", "Anger", "Boredom", "Sleepiness", "Sadness", "Excitement", "Calm", and "Happiness". Similarly to the first experiment, suitable labels for representing emotions were chosen that are common with the Swedish labels of Knez and Hygge [127] and with the Portuguese labels presented in [48] and [143], coupled with the resulting end-point labels from the first experiment. We decided to select for Portuguese the words: "Medo", "Surpresa", "Aversão", "Raiva", "Aborrecimento", "Sonolência", "Tristeza", "Excitação", "Calmo", and "Felicidade". On the other hand, for Swedish, we decided to select the words: "Rädsla", "Överraskad", "Avsky", "Ilska", "Uttråkad", "Trött", "Ledsen", "Spänning", "Lugn", and "Lycka". Due to our approach to minimize bias while recognizing prior research, we believe a suitable set of labels is selected.

\section{Hypothesis}

The main hypothesis for this second experiment is that the levels of pleasure and arousal associated with an emotional label depends on the culture and language. Therefore, the same pleasure and arousal values cannot be used to represent emotion words/labels translated from one language to another.

\section{Participants}

In this second experiment, the Prolific platform was also used to recruit participants by applying the same demographic, language, age, and approval rate filters as in the first experiment.

In the experiment with Portuguese participants, 50 women and 50 men ranging in age from 18 to 52 years old $(\mu=24.10, \sigma=5.91)$ were recruited. The mean of the approval rate for Portuguese participants was 99.06 with a minimum of 95 and a mean of 82.44 studies performed successfully.

For the Swedish experiment, 50 women and 50 men ranging in age from 18 to 67 years old $(\mu=30.79, \sigma=10.37)$ were recruited. The mean of the approval rate for Swedish participants was 99.39 with a minimum of 94 and a mean of 80.19 studies performed successfully. 


\section{Materials}

To determine the values associated with the variables of pleasure and arousal in a target language, we designed an online questionnaire composed of ten prompts, one for each emotion label. Each prompt followed the same structure: the title of the prompt contained only an emotion label; then, two 7-item Likert scales were used to assign the levels of pleasure and arousal associated with the emotion label. The arousal scale comprised values from Very sleepy to Very aroused and the pleasure scale comprised values from Very miserable to Very pleased. Note that, in both scales, the value 4 corresponded to the neutral value. In addition, an attention check question was shuffled along with the ten prompts of the experiment. In this attention check question, participants were asked to assign to the label "Warm" a value between very cold and very warm using a 7-item Likert scale as in the rest of the questionnaire prompts.

\section{Procedure}

As in the first experiment, participants had to enter their Prolific ID to register for the questionnaire. At the beginning of the experiment, an explanation of the objective of the experiment together with a brief introduction to the dimensions of pleasure and arousal was given to the participants. In addition, instructions describing the task to be performed were also provided. Participants were then asked to complete the ten questions of the experiment and the attention check question with no time limit.

\subsection{Results}

\section{First experiment}

To measure the suitability of a word $w$ to represent the translation of an affective category $c$ into a target language, we use the degree of acceptance. The degree of acceptance $\left(\operatorname{accept}_{c}(w)\right)$ is defined as the difference between the number of times the word $w$ has been selected as representative for the 


\subsection{RESULTS}

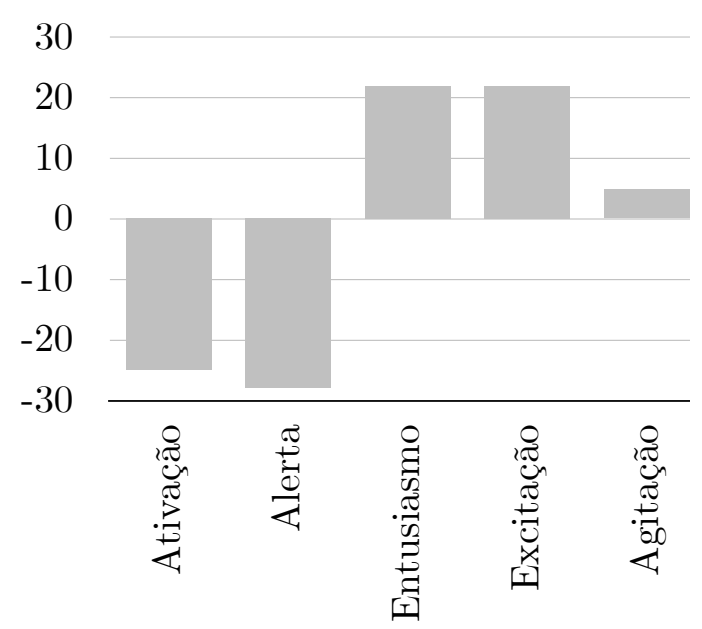

(a) Arousal

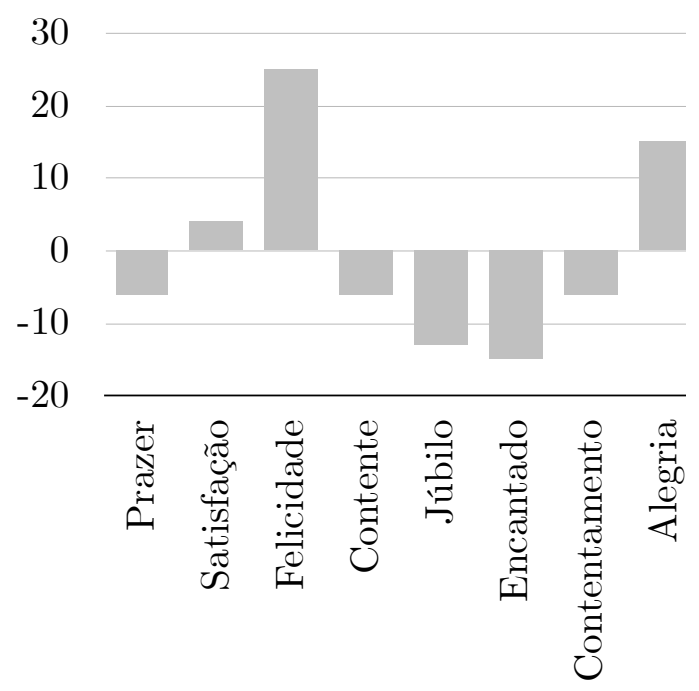

(c) Pleasure

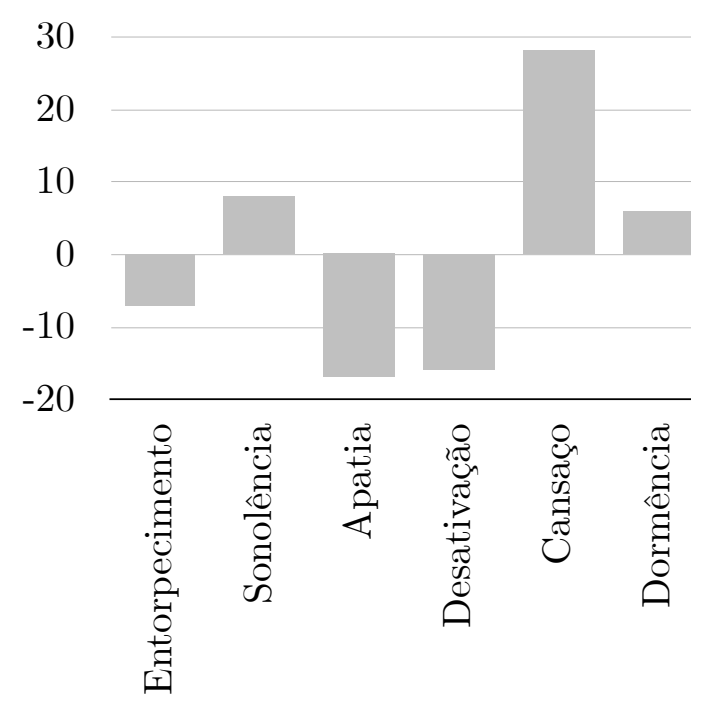

(b) Sleepiness

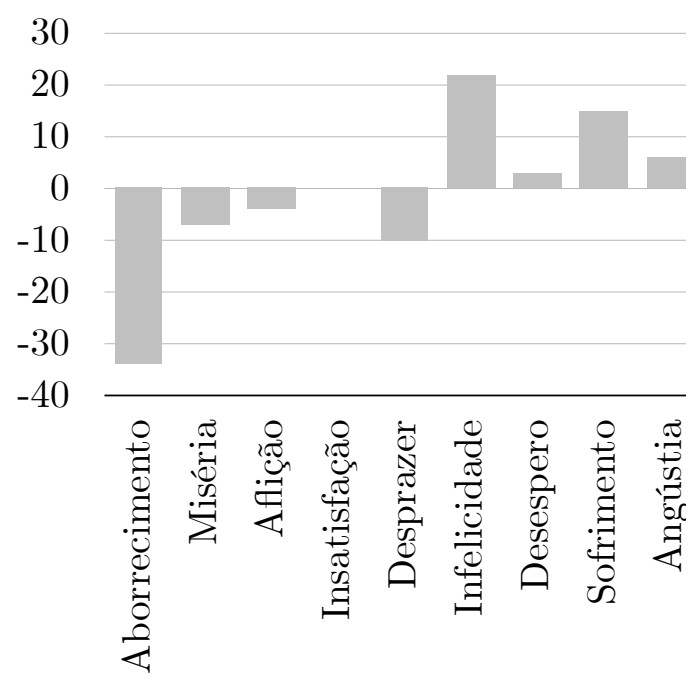

(d) Misery

Figure 3.2: Degree of acceptance of the candidate words in Portuguese for each affective category. 


\section{CHAPTER 3. COMPUTATIONAL AFFECTIVE KNOWLEDGE REPRESENTATION}

affective category $c$ and the number of times the word has been selected as non-representative of that affective category.

\section{Portuguese experiment}

Figure 3.2 shows the degree of acceptance of the candidate words for the four affective categories. For example, in the case of the affective category misery, the degree of acceptance for "Infelicidade" was accept $t_{\text {misery }}($ Infelicidade $)=$ 22 indicating that the participants considered this word as very representative for that affective category. In contrast, the degree of acceptance for "Aborrecimento" was accept $t_{\text {misery }}($ Aborrecimento $)=-34$ indicating that the majority of the participants rejected this word to represent the affective category of the group. Based on the participants' choices in each affective category (see Figure 3.2), we have obtained the following words with a positive degree of acceptance: for pleasure participants chose the words "Felicidade", "Alegria", and "Satisfação"; for misery the words "Infelicidade", "Sofrimento", "Angústia", and "Desespero" were chosen; for arousal the selected words were "Entusiasmo", "Excitação", and "Agitação"; and for sleepiness the words chosen were "Cansaço", "Sonolência", and "Dormência".

In the case of the label pleasure (Figure 3.2c), the words "Júbilo", "Contentamento", and "Prazer" had a negative degree of acceptance. For the label misery (Figure 3.2d), "insatisfação" has a neutral degree of acceptance and "Aflição", "Miséria", "Aborrecimento", and "Desprazer" has a negative degree of acceptance. The label arousal (Figure 3.2a) has two words with a degree of acceptance "Ativação" and "Alerta". Finally, the label sleepiness (Figure 3.2b) has three words with negative degree of acceptance "Apatia", "Desativação", and "Entorpecimento".

The labels generally used for the affective category of pleasure in previous Portuguese studies [48, 143] are "Prazer" for pleasure and "Desprazer" for misery (displeasure), which was an expected result of the first experiment in Portuguese, which could capture a general meaning of pleasure and misery. However, these words were not chosen by the participants. Intuitively, this result is due to the less intensive meaning of the word "Prazer" in comparison to "Felicidade" and "Alegria" (chosen by the participants), and the less in- 
tensive meaning of the word "Desprazer" in comparison to "Infelicidade" and "Sofrimento" (chosen by the participants). From the experiment results, and by being strict to our unbiased approach, it would not be numerically possible to include the word "Prazer" in Figure 3.2c and the word "Desprazer" in Figure $3.2 \mathrm{~d}$ because these two words have a negative degree of acceptance by the participants.

Other labels chose by the participants, such as "Sonolência", "Cansaço", "Entusiasmo", and "Contente", are shared with the Portuguese labels of [143], which can suggest their suitability. However, the labels "Dormência", "Agitação", "Desespero" where not included in that study. Intuitively, this result is due to the more intensive or clear meanings of these labels than some of their synonyms, e.g., "Preguiçoso", "Tenso", "Chateado", respectively, included in the study by [143].

In order to perform the second experiment, we decided to select for the affective categories arousal, sleepiness, and pleasure, those words with a positive degree of acceptance, obtaining three words for each affective category. However, by applying the same criteria for misery, four possible words were obtained. To avoid bias and misunderstandings, we decided to maintain a homogeneous number of words for each affective category and, therefore, for the affective category misery we selected the three words with the highest level of acceptance.

\section{Swedish experiment}

The results obtained for the first experiment conducted with Swedish participants are shown in Figure 3.3. Analyzing the degree of acceptance of these results, we can appreciate that there is a larger set of words with positive degree of acceptance than in the case of the experiment conducted with Portuguese participants. However, it is important that none of the sets have terms that may be contradictory or confusing. If this happens we have two options: we can select three words instead of two (to increase the context) or we can select only one word (to reduce confusion). We ended up using two words for each end-point, which we believe resulted in suitable sets of labels (with sufficient contextual space and minimal confusion) for the second exper- 


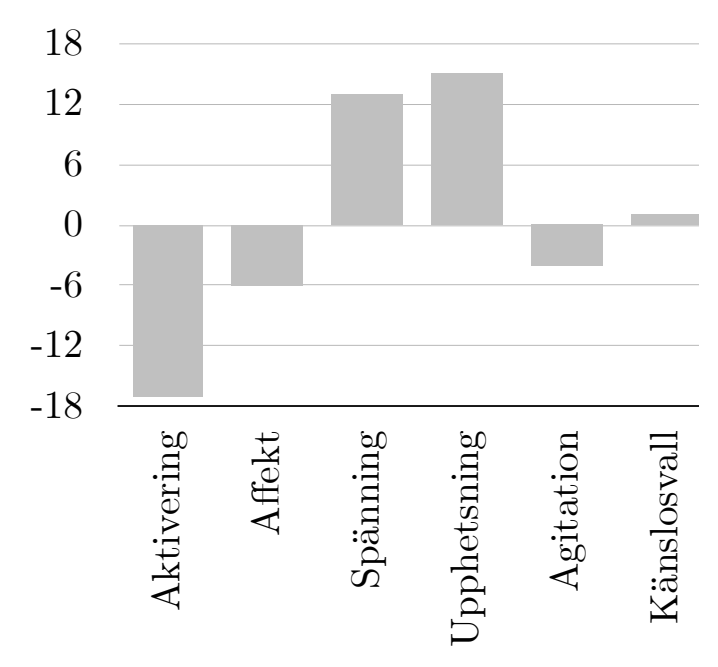

(a) Arousal

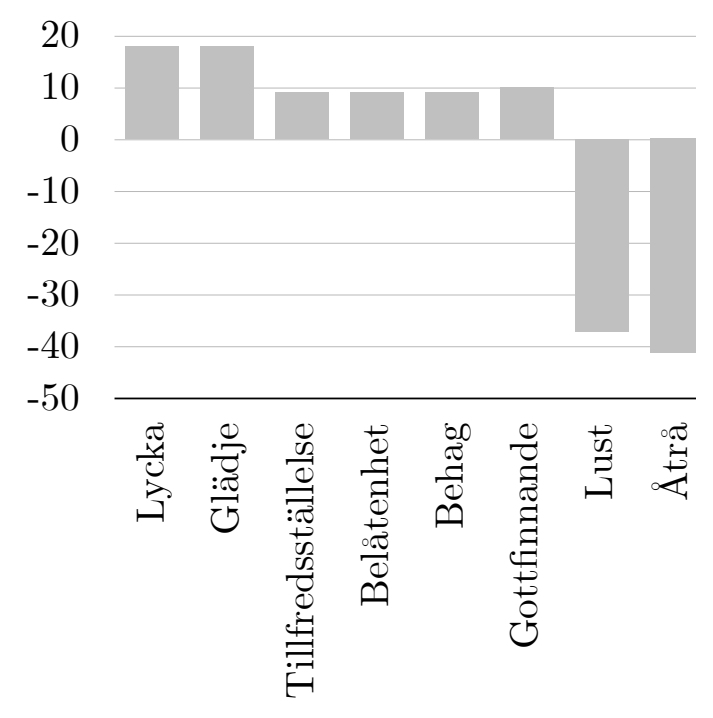

(c) Pleasure

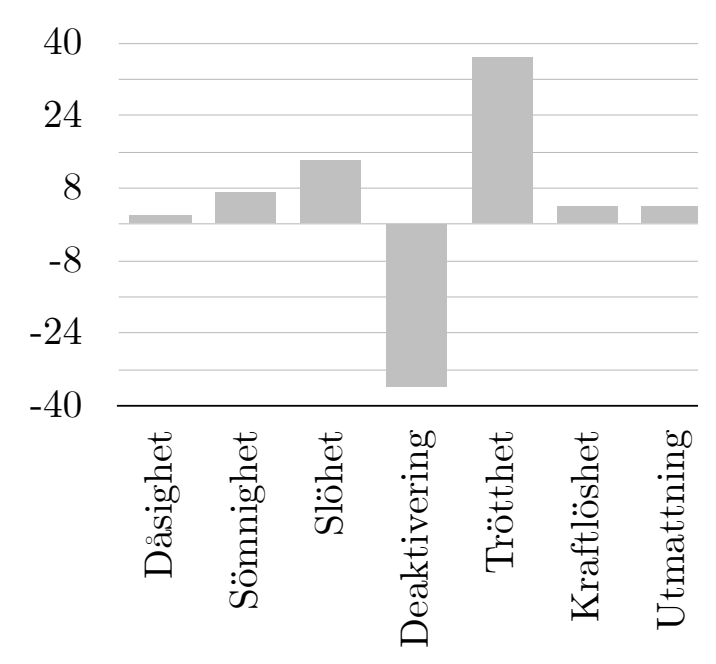

(b) Sleepiness

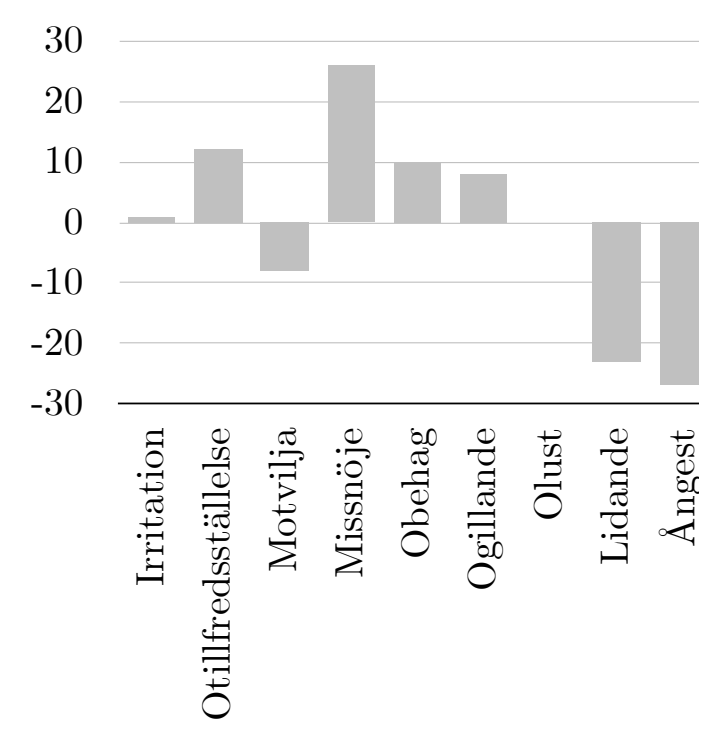

(d) Misery

Figure 3.3: Degree of acceptance of the candidate words in Swedish for each affective category. 


\subsection{RESULTS}

iment. Therefore, based on the participants' choices, this resulted in "Lycka" and "Glädje" for pleasure, "Otillfredsställelse" and "Missnöje" for misery, "Spänning" and "Upphetsning" for arousal, and "Slöhet" and "Trötthet" for sleepiness.

Commonly used labels for hedonic tone (pleasure/misery) in prior Swedish studies and literature [211, 120] are "Behag" for pleasure and "Obehag" for misery (displeasure). This was an expected result of the first Swedish experiment, which arguably would capture a general meaning of pleasure and misery. However, these words were not chosen by the participants. Intuitively, this result is due to the less intensive meaning of the word "Behag" in comparison to "Lycka" and "Glädje" (chosen by the participants), and the less intensive meaning of the word "Obehag" in comparison to "Otillfredsställelse" and "Missnöje" (chosen by the participants). From the results of the experiment, and by being strict to our unbiased approach, it would not be numerically possible to include the word "Behag" in Figure 3.3c and the word "Obehag" in Figure 3.3d without also including words in other sets that could increase confusion. This result reinforced our decision using two words for each end-point.

Other labels chosen by the participants, such as "Lycka", "Glädje", "Slöhet", and "Trötthet", are common with the Swedish labels of Knez and Hygge [127], which can suggest their suitability. However, the labels "Missnöje", "Spänning", "Upphetsning" where not included in the study by Knez and Hygge. Intuitively, this result is due to the more intensive or clear meanings of these labels than some of their synonyms, e.g., "Besvärad", "Upprymd" and "Begeistrad", respectively, included in the study by Knez and Hygge. More intensive and clear labels for end-points have been observed to have clearer conceptions [15], which can suggest their suitability to be used as pleasure and arousal end-points.

\section{Second experiment}

To analyse the results of this second experiment, we will refer only to the dimensions of Pleasure and Arousal, considering that Misery is negative Pleasure and Sleepiness is negative Arousal. 


\section{CHAPTER 3. COMPUTATIONAL AFFECTIVE KNOWLEDGE REPRESENTATION}

Table 3.1: Circular mean and circular standard deviation in degress for each emotion in Portuguese, Swedish, and Spanish languages.

\begin{tabular}{|c|c|c|c|c|c|c|c|c|c|}
\hline \multirow{2}{*}{$\begin{array}{c}\text { Emotions in English } \\
\text { Label/word }\end{array}$} & \multicolumn{3}{|c|}{ Emotions in Portuguese } & \multicolumn{3}{|c|}{ Emotions in Swedish } & \multicolumn{3}{|c|}{ Emotions in Spanish } \\
\hline & Label/word & Mean & $\mathrm{Sd}$ & Label/word & Mean & $\mathrm{Sd}$ & Label/word & Mean & $\mathrm{Sd}$ \\
\hline Fear & Medo & 160.12 & 39.25 & Rädsla & 136.93 & 21.62 & Miedo & 125.51 & 15.61 \\
\hline Surprise & Surpresa & 62.79 & 21.02 & Överraskad & 75.06 & 18.86 & Sorpresa & 71.63 & 26.38 \\
\hline Disgus & Aversão & 195.37 & 41.88 & Avsky & 167.88 & 34.00 & Asco & 182.58 & 43.65 \\
\hline Anger & Raiva & 151.60 & 37.72 & & & 8.20 & ado & & 16.90 \\
\hline & & 245.96 & 21. & & & 9. & & 24 & 21.41 \\
\hline Sleep & Sonolê & 259.19 & $18 .($ & Trött & 254.39 & 16.79 & Somnolencia & 263.59 & 15.56 \\
\hline Sadne & Trister & 207.78 & 15.51 & Ledsen & 205.69 & 20.31 & Tristeza & 196.02 & 22.48 \\
\hline Excitement & Excitação & 53.18 & 8.64 & Spänning & 76.78 & 20.96 & Emoción & 39.97 & 10.32 \\
\hline Calm & Calmo & 312.68 & 46.76 & Lugn & 325.44 & 37.77 & Calma & 318.12 & 35.89 \\
\hline Happiness & Felicidade & 40.22 & 7.66 & Lycka & 32.66 & 15.64 & Felicidad & 25.09 & 19.02 \\
\hline
\end{tabular}

In a previous analysis of the results, those responses in which the participants selected both neutral values (i.e., the $4^{\text {th }}$ item in both Likert scales) in the pleasure and arousal dimensions were removed. This decision was made according to previous studies in which it was evidenced that, at values close to zero in both dimensions, the intensity of emotions is so low that it can be considered that there is no emotion [183]. Then, we scaled the obtained responses so that the $1^{\text {st }}$ item of the Likert scale corresponds to the value -1 and the $7^{\text {th }}$ item of the Likert scale to the value 1 of the coordinate axis. Note that the $4^{\text {th }}$ item in the Likert scale corresponds to the value 0 (i.e. the origin of coordinates of the polar representation). Once scaled, we calculated the angle of each response from the pleasure and arousal values. This angle represents an approximation to the conceptualization that each participant associates with an emotion in the pleasure and arousal space. Finally, we estimated the dispersion of the samples for each emotion in each of the two languages and we removed the possible outliers.

Table 3.1 summarizes the results obtained in the second experiment in the Portuguese and Swedish languages, as well as the results of the previous experiment conducted with Spanish-speaking European participants presented in [220]. This table presents the mean angle for each emotion together with its circular standard deviation obtained using the Formula 3.1. To facilitate the visualization and the analysis of the distribution of the ten emotions in the circular space composed of the pleasure and arousal dimensions, we proposed 


\subsection{RESULTS}

the use of the circular boxplots [36] that can be seen in Figures 3.4 and 3.5 for Portuguese and Swedish respectively.

\section{Portuguese experiment}

The values of pleasure and arousal associated with the 10 emotions in Portuguese resulting from the second experiment can be seen in Table 3.1.

In this experiment, some emotions had a much larger standard deviation than others. It can be a result of either unclear labelling or that the participants had a clearer conception of some emotions, making it easier to define them in terms of pleasure and arousal. For instance, "Excitement" and "Happiness" had more clear responses from the participants, while emotions such as "Calm" and "Fear" seemed to be more challenging to define. This goes in line with previous research, which has observed that stronger/intense emotions, such as "Happiness", have more precise conceptions (emotional differentiation). Similar observations could be made when comparing languages. For some emotions, the standard deviation had apparent differences between the Portuguese test and the Swedish test. The Portuguese test had much clearer "Excitement", "Happiness", and "Sleepiness" definitions. Intuitively, it can be that these emotion labels have more precise definitions in Portuguese. Comparing the Swedish, Spanish, and Portuguese emotion measures, we can observe similarities on "Excitement", "Boredom", and "Sleepiness" on Portuguese and Spanish measures (Table 3.4).

Based on Table 3.1 and Figures 3.4 and 3.7, we can observe: i) positive arousal and positive pleasure are related to the emotions "Happiness", "Excitement", and "Surprise"; ii) positive arousal and negative pleasure are related to "Anger" and "Fear"; iii) negative arousal and negative pleasure are related to "Disgust", "Boredom", "Sleepiness"; and iv) negative arousal and positive pleasure are related to "Calm".

Furthermore, we can verify that they keep their values in the quadrants, considering the median and the standard deviations "Happiness" and "Excitement" (positive arousal and pleasure). For the other emotions, and based on Figure 3.4, 75\% of the results belong to the quadrant of the median of each emotion, but $25 \%$ of the results belong to different quadrants. 


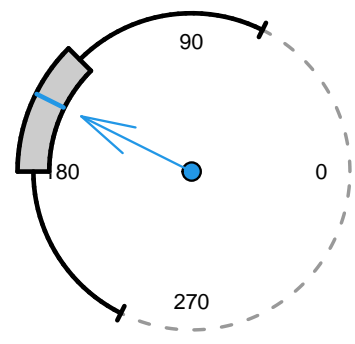

(a) Fear

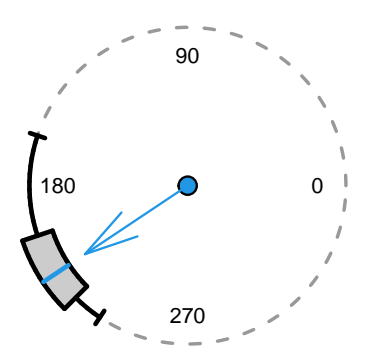

(d) Sadness

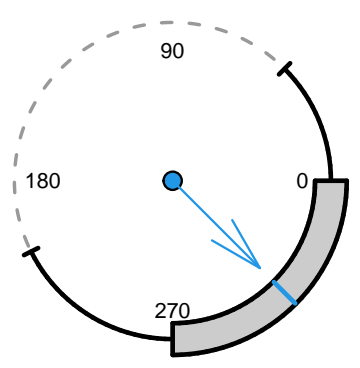

(g) Calm

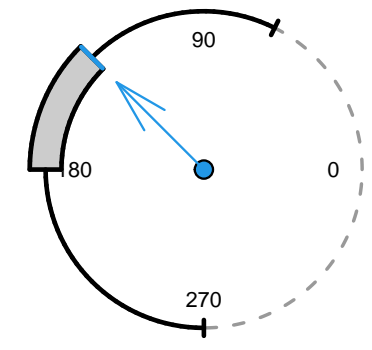

(b) Anger

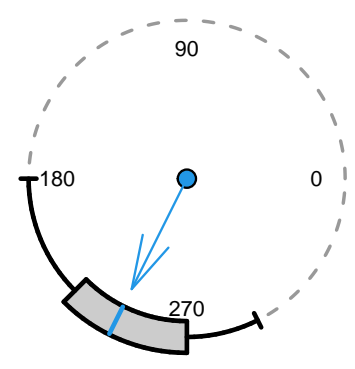

(e) Boredom

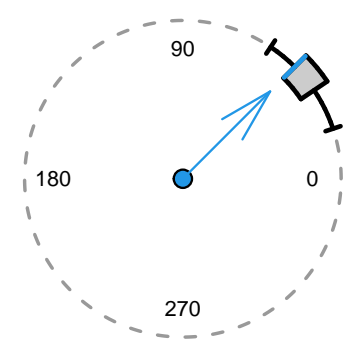

(h) Happiness

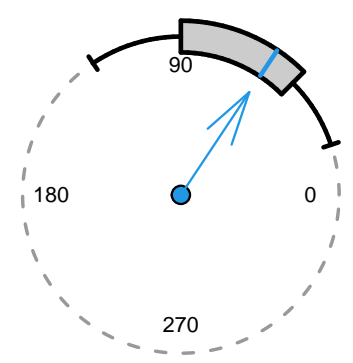

(j) Surprise

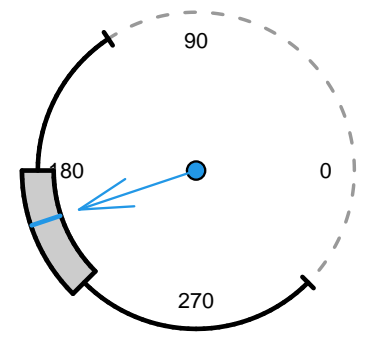

(c) Disgust

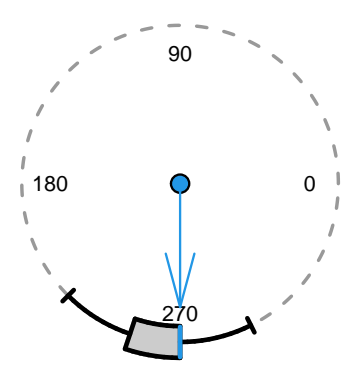

(f) Sleepiness

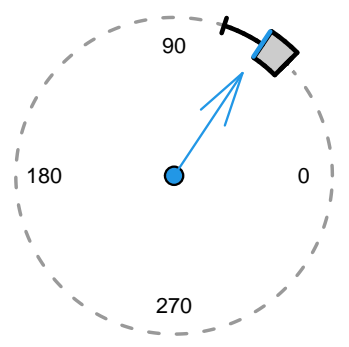

(i) Excitement

Figure 3.4: Portugal experiment results. 


\subsection{RESULTS}

According [222], the proximity or distance between the emotions represented circumference assume the similarity or difference between emotions. The model defines that emotions are less positively correlated when they are approximately 90 degrees apart. At 90 degrees apart, two affective states should be very little or not correlated. In turn, at 180 degrees of distance, affective states must be negatively correlated. Thus, based on Figure 3.7a), we can verify that "Sleepiness" / "Boredom" is negatively correlated with "Excitement" / "Happiness".

\section{Swedish experiment}

Similar to the Portuguese experiment, in the second Swedish experiment, some emotions had a much larger standard deviation than others. Once again, this can be a result of either unclear labelling or that the participants had a more clear conception about some emotions, making it easier to define them in terms of pleasure and arousal. For instance, "Anger" and "Boredom" had more clear responses from the participants, while emotions such as "disgust" and "Calm" seemed to be more difficult to define. This also goes in line with previous research, which has observed that stronger/intense emotions, such as "Anger", have clearer conceptions (emotional differentiation) [15].

Similar observations could be made when comparing languages. For some emotions, the standard deviation had clear differences between the Swedish test and the Portuguese test. A reason for this can be cultural as well as personal. For instance, "Excitement" (translated to "Spänning" in Swedish) that in English commonly is perceived as a positive emotion, can in Swedish (depending on the context) be perceived as either negative (e.g., too exciting or a scary level of thrill) or positive (e.g., having fun or expecting/waiting for something good). This is observed in the Swedish test result, where "Excitement" spans from pleasure to displeasure, although pleasure is dominant (see Figure 3.6). The Portuguese test, however, had much clearer "Excitement" definitions, being strictly pleasurable (see Figure 3.6). Other emotions, such as "Boredom" and "Anger" had much smaller standard deviations in the Swedish test compared to the Portuguese test (see Figure 3.4 and 3.5). Intuitively, it can be that these emotion labels have more precise definitions in 


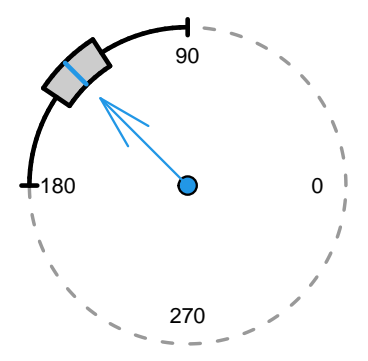

(a) Fear

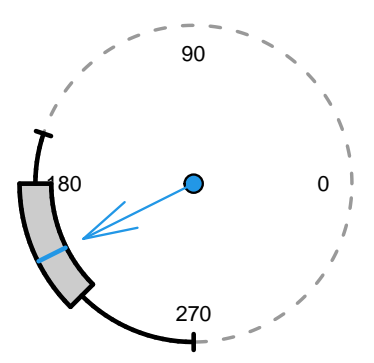

(d) Sadness

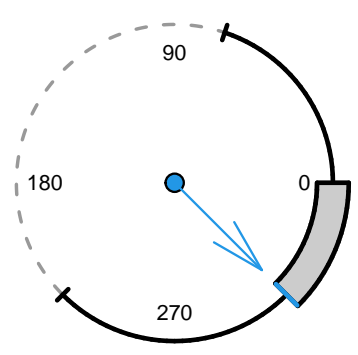

(g) Calm

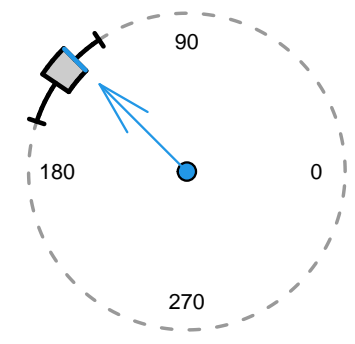

(b) Anger

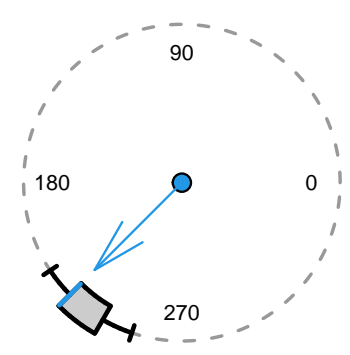

(e) Boredom

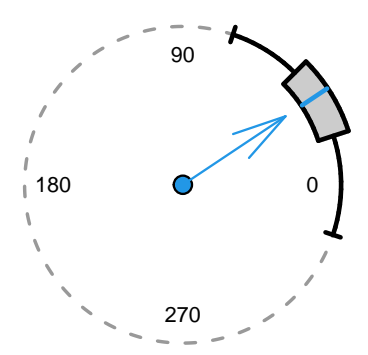

(h) Happiness

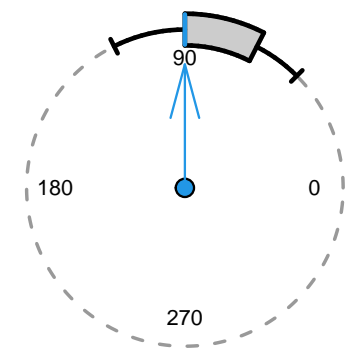

(j) Surprise

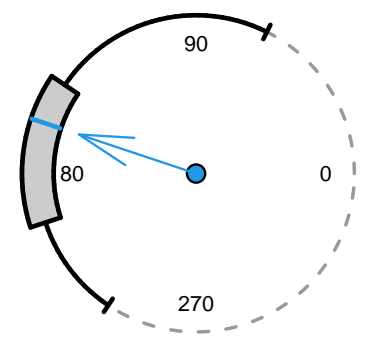

(c) Disgust

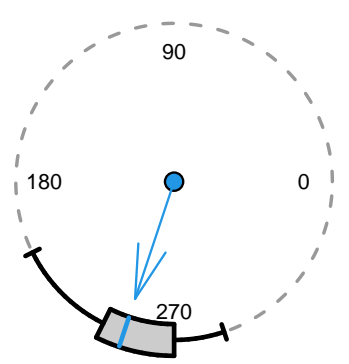

(f) Sleepiness

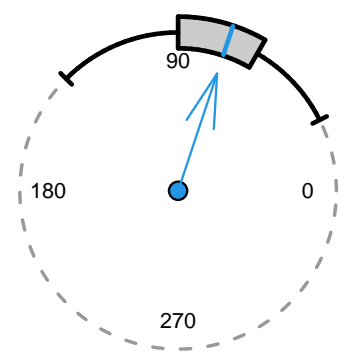

(i) Excitement

Figure 3.5: Sweden experiment results. 
Swedish.

\subsection{Discussion}

\section{Comparison between languages}

Analyzing the results of the first experiment shown in Figure 3.2, the Portuguese label "Prazer" (pleasure) has a negative impact, which may be explained based on the cultural and religious aspect, because on Portuguese meaning, "Prazer" (pleasure) is associated to sexual pleasure instead of happiness and have a negative opinion on society. In the same way, the label "Desprazer" (displeasure) also have a negative impact on the label choice, which sustain the previous explanation.

From the second experiment, comparing the Portuguese and Swedish emotion measures (see Figure 3.4 and 3.5), we can observe a small decrease in arousal and pleasure for the Portuguese on all "negative" emotions (i.e., "Fear", "Anger", "Disgust", "Sadness", "Boredom", and "Sleepiness"), approximately increasing the angle representation of each Portuguese emotion with 5-10 degrees. While the same could not be observed for more "positive" emotions (i.e., "Excitement", "Happiness", and "Surprise"), an interesting point is that the difference is similar/consistent for all emotions in set "negative" emotions. Furthermore, in Figure 3.7, we can observe that "Fear" and "Anger" have less intense arousal for Portuguese results on the second experiment than Spanish and Swedish results. Also, for "Happiness", the Portuguese result is more intense in terms of pleasure and arousal. Finally, the "Calm" emotion has less intense pleasure than Spanish and Swedish, but the arousal intensity is similar to the Swedish results.

From these observations we may conclude that: i) there is a cultural difference in the subjective experience of these emotions, and ii) there seems to be a cultural modifier that is consistent across emotions. We may thus make a third conclusion: iii) we are measuring similar emotions in the Swedish experiment and the Portuguese experiment, if normalized with the observed cultural modifier. This is interesting from a modelling perspective. If able 


\section{CHAPTER 3. COMPUTATIONAL AFFECTIVE KNOWLEDGE REPRESENTATION}

\begin{tabular}{|c|c|c|c|c|}
\hline $\begin{array}{l}-\bullet \text { Happiness } \\
-x-\text { Excitement }\end{array}$ & $\begin{array}{l}-\oplus-\text { Fear } \\
-\Delta-\text { Surprise }\end{array}$ & $\begin{array}{l}\text { - - - Disgust } \\
\text {-*- Anger }\end{array}$ & $\begin{array}{l}-\square-\text { Boredom } \\
-\nabla-\text { Sadness }\end{array}$ & $\begin{array}{l}-+- \text { Calm } \\
-\square-\text { Sleepiness }\end{array}$ \\
\hline
\end{tabular}

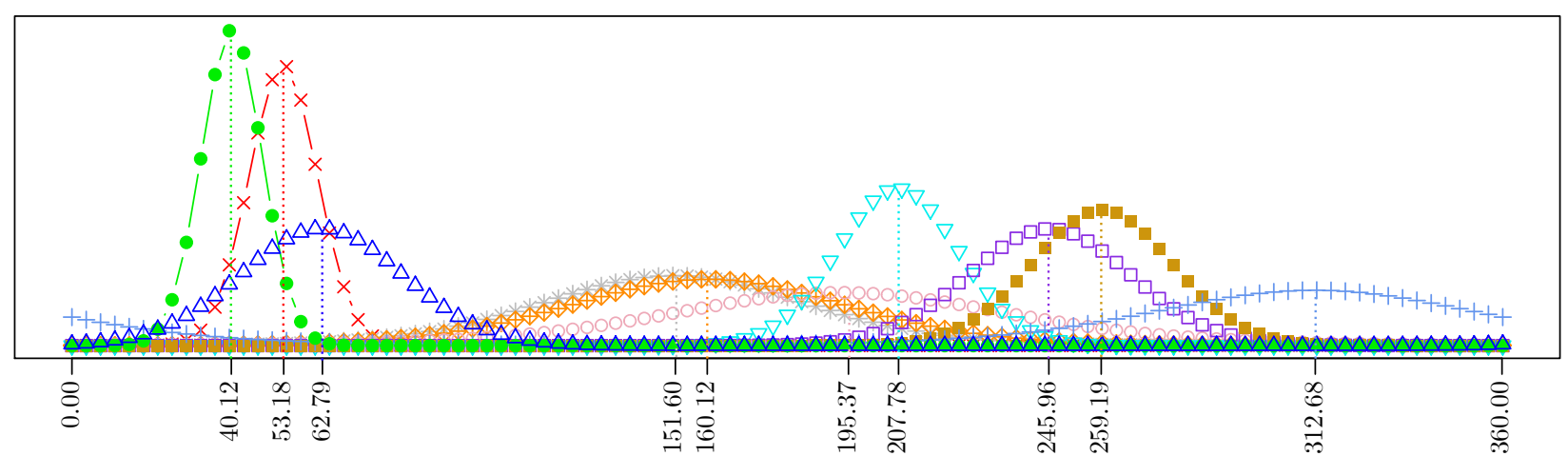

(a) Portuguese

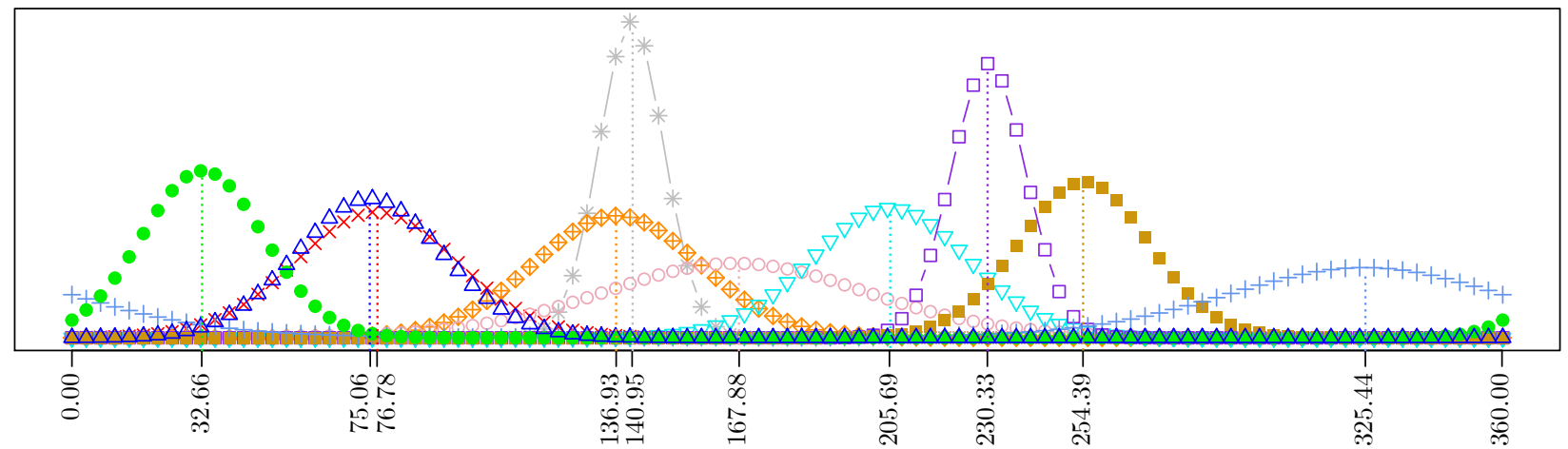

(b) Swedish

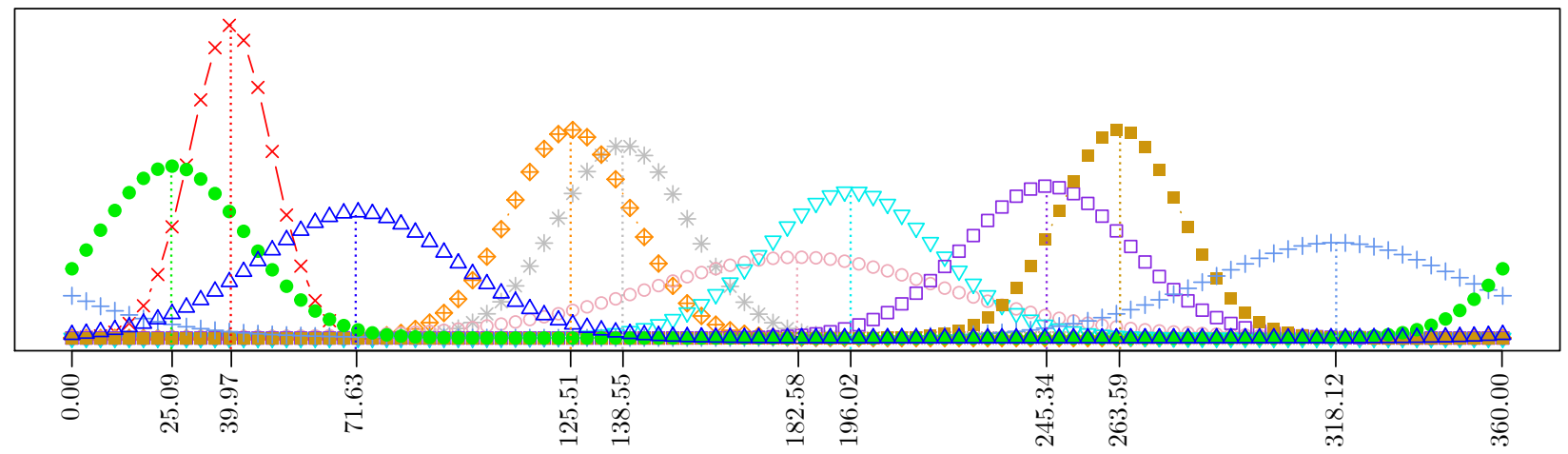

(c) Spanish

Figure 3.6: Mixture of Von Mises curves of the ten emotions in Portuguese, Swedish, and Spanish. The axis represents the angles $\alpha_{e}$ in degrees. 


\subsection{DISCUSSION}

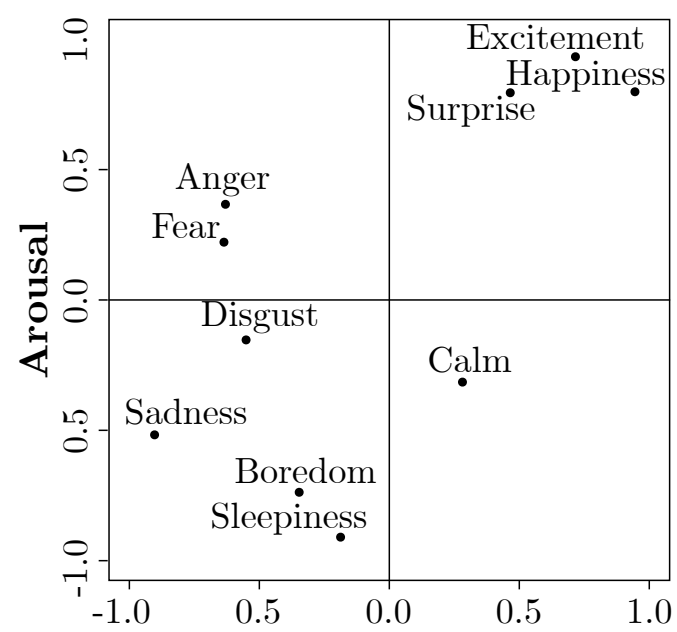

(a) Portuguese

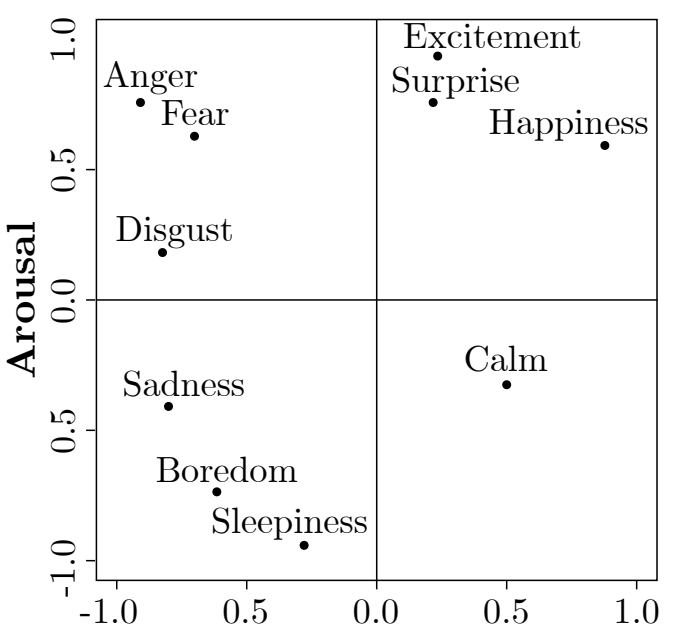

(b) Swedish

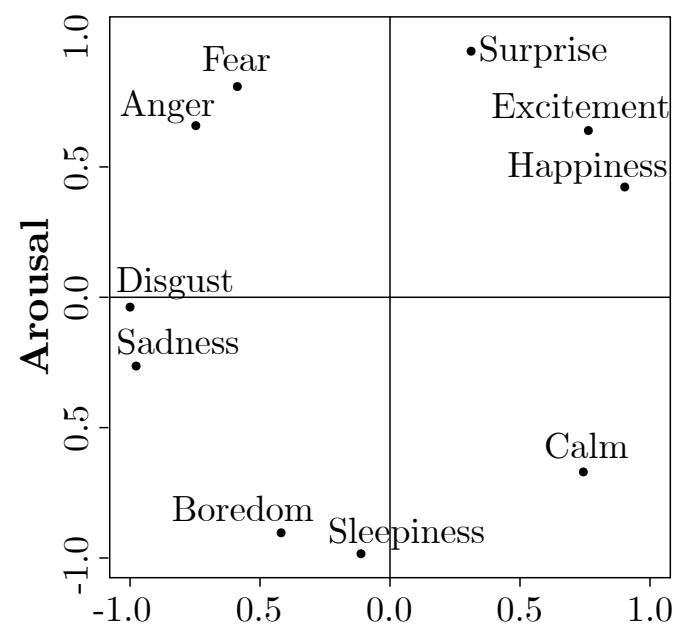

(c) Spanish

Figure 3.7: Results obtained. 


\section{CHAPTER 3. COMPUTATIONAL AFFECTIVE KNOWLEDGE REPRESENTATION}

Table 3.2: Results of the chi-square test for the comparison of the distribution of emotions across languages. Note that $*$ indicates p-value $<0.05$.

\begin{tabular}{lrrrrrrrrr}
\hline & \multicolumn{2}{c}{ Portuguese - Swedish } & & \multicolumn{2}{c}{ Portuguese - Spanish } & & \multicolumn{2}{c}{ Swedish - Spanish } \\
\cline { 2 - 3 } \cline { 8 - 9 } Emotion & $\chi^{2}$ value $(\mathrm{df})$ & $\mathrm{p}$-value & & $\chi^{2}$ value $(\mathrm{df})$ & p-value & & $\chi^{2}$ value $(\mathrm{df})$ & p-value \\
\hline Fear & $45.899(15)$ & $0.000^{*}$ & & & $75.95(15)$ & $0.000^{*}$ & & $23.951(8)$ & $0.002^{*}$ \\
Surprise & $21.884(10)$ & $0.015^{*}$ & & $30.313(12)$ & $0.002^{*}$ & & $23.119(11)$ & $0.017^{*}$ \\
Disgust & $53.519(18)$ & $0.000^{*}$ & & $19.224(18)$ & 0.378 & & $56.231(18)$ & $0.000^{*}$ \\
Anger & $54.674(14)$ & $0.000^{*}$ & & $25.506(14)$ & $0.029^{*}$ & & $31.708(7)$ & $0.000^{*}$ \\
Boredom & $56.461(8)$ & $0.000^{*}$ & & $3.314(8)$ & 0.913 & & $58.382(9)$ & $0.000^{*}$ \\
Sleepiness & $8.8291(7)$ & 0.265 & & $12.584(7)$ & 0.082 & & $21.064(8)$ & $0.006^{*}$ \\
Sadness & $18.458(9)$ & $0.030^{*}$ & & $22.028(11)$ & $0.024^{*}$ & & $24.191(13)$ & $0.029^{*}$ \\
Excitement & $83.071(9)$ & $0.000^{*}$ & & $61.470(7)$ & $0.000^{*}$ & & $121.82(12)$ & $0.000^{*}$ \\
Calm & $26.338(15)$ & $0.034^{*}$ & & $16.788(12)$ & 0.157 & & $12.567(14)$ & 0.560 \\
Happiness & $22.512(8)$ & $0.004^{*}$ & & $43.523(7)$ & $0.000^{*}$ & & $20.367(9)$ & $0.015^{*}$ \\
\hline
\end{tabular}

to measure and identify cultural modifiers for the experience of emotions (independent of particular emotions), these can work as heuristics in shared representations of emotions across cultures.

We performed a comparative analysis of the Portuguese, Swedish, and Spanish samples to identify the existence of possible discrepancies between languages when assessing the levels of pleasure and arousal for the ten emotions. Since the nature of the experiment implies that we have a finite number of possible combinations of responses and, consequently, we have a finite number of possible angles. Therefore, we decided to apply a Chi-square test to test whether there were significant differences between language and the angles associated with emotions. The starting hypotheses for this analysis are: the null hypothesis $h_{0}$ "the angle associated with an emotion is independent of culture and language"; and the alternative hypothesis $h_{1}$ "the angle associated with an emotion and culture and language have some degree of dependence".

Table 3.2 shows the results of this analysis. When testing whether there is a dependence between Portuguese and Swedish we can appreciate that, except for the case of "Sleepiness", we can reject the null hypothesis $h_{0}$ with a confidence level of $95 \%$, i. e., there is a language dependence in the selection 


\subsection{DISCUSSION}

of emotion angles. In other words, for nine of the ten emotions, the differences between the emotion angles are statistically significant between languages. The same applies when comparing Swedish with Spanish. As we can see, there is statistically significant evidence that the selection of the emotion angle depends on language in nine of the ten emotions (all except "Calm"). However, when comparing Portuguese with Spanish, we can observe that these differences are reduced. In this case, we find four emotions ("Disgust", "Boredom", "Sleepiness", and "Calm") for which we do not have enough evidence to reject the null hypothesis $h_{0}$ with a confidence level of $95 \%$. These results are interesting, since at the linguistic and cultural level, there are greater similarities between Portuguese and Spanish than between Portuguese and Swedish or Spanish and Swedish. Considering the fact that Portuguese and Spanish share a greater lexical similarity since both are included in the Ibero-Romance language family group [168], these results are in line with the results obtained in previous work with the study developed in [107] (described in Section 3.2) in which similarities between language families were observed.

\section{A cross-cultural model of affect adapted to Portuguese and Swedish}

As discussed in Section 3.3, although a dimensional model provides a general representation of affective states, it is necessary to adapt the representation of affective labels to the language in which the model is going to be used. For this purpose, from the results obtained in the experiments with Portuguese and Swedish participants, we estimated the probability regions for the ten emotions in the pleasure and arousal space using the Von Mises distribution described in Formula 3.3. Figure 3.6 shows the estimated Von Mises distributions for the ten emotions in Portuguese, Swedish, and Spanish. From these distributions, we have generated an emotional representation model for the Portuguese and Swedish languages following the model presented in Section 3.3 for the Spanish language. Figures 3.8 and 3.9 show a graphical representation of the probability regions for the Portuguese and Swedish affect representation models using one standard deviation. As in Figure 3.1, the outer rings of Figures 3.8 and 3.9 represent the regions assigned to each emo- 


\section{CHAPTER 3. COMPUTATIONAL AFFECTIVE KNOWLEDGE}

REPRESENTATION

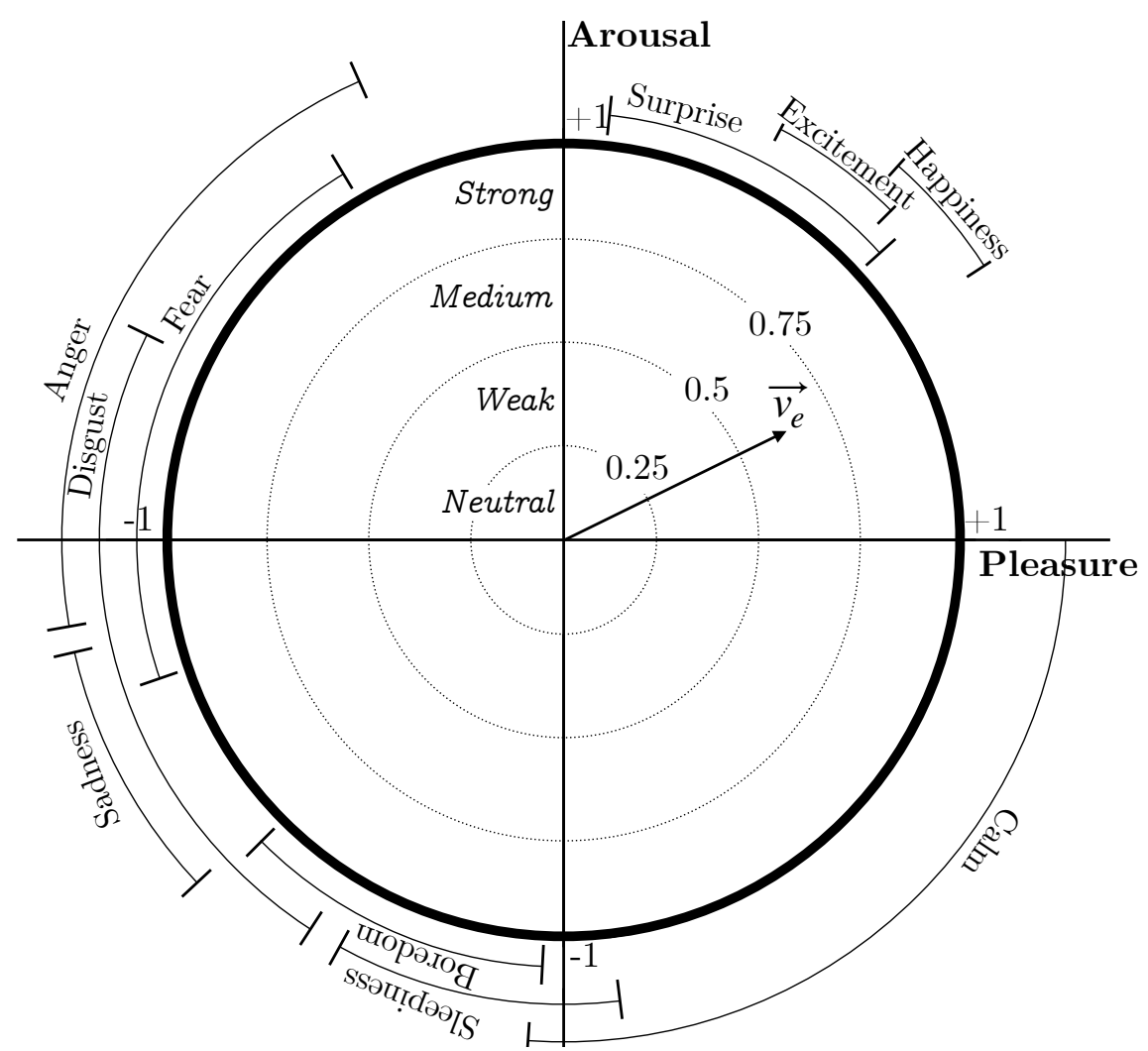

Figure 3.8: Distribution of probability functions in the pleasure-arousal space using one standard deviation for Portuguese.

Table 3.3: Example of the results of the Emotion Representation process for the emotion "Happiness" with a Medium intensity.

\begin{tabular}{lccrr}
\hline & $\alpha$ & $|v|$ & Pleasure & Arousal \\
\hline Portuguese & $40.22^{\circ}$ & 0.625 & 0.477 & 0.404 \\
Swedish & $32.66^{\circ}$ & 0.625 & 0.526 & 0.337 \\
Spanish & $25.09^{\circ}$ & 0.625 & 0.566 & 0.265 \\
\hline
\end{tabular}

tion in each language, while the inner rings represent the different levels of intensity.

Once defined the representation models of affect for Portuguese and Swedish, to allow the representation, interpretation, and expression of affective states using a polar representation, we designed two processes (described in detail in 


\subsection{DISCUSSION}

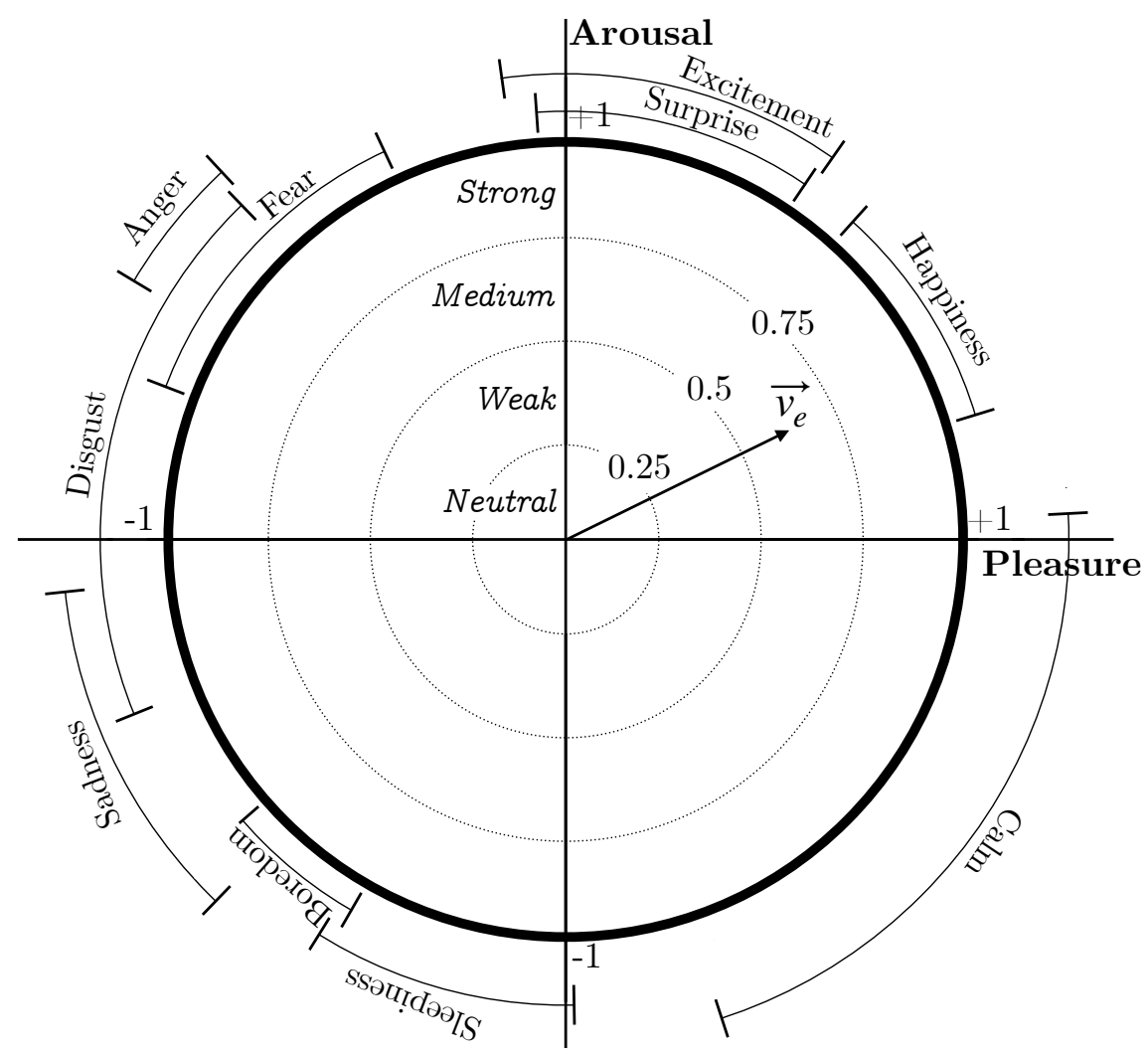

Figure 3.9: Distribution of probability functions in the pleasure-arousal space using one standard deviation for Swedish.

[220]): the Emotion Representation process and the Emotion Expression process. The Emotion Representation process is a defuzzification process that, based on the mean values obtained in the experiments for each emotion, allows the agent to represent in the pleasure-arousal space the emotions recognized or internally elicited as part of an affective process (e.g. appraisal process). For example, when an affective agent needs to represent the emotion "Happiness" with a Medium intensity, the Emotion Representation process uses the mean angle $\alpha$ of the emotion "Happiness" adapted to the cultural environment to estimate the angle of the vector $\alpha$. To estimate the modulus of the vector $|v|$ we use the midpoint of each intensity region. In the example, the Medium intensity corresponds to 0.625 (the midpoint between 0.5 and 0.75 ) (see Figure 3.8). Table 3.3 shows the values of pleasure and arousal when applying the Emotion Representation process to emotion "Happiness" with 


\section{CHAPTER 3. COMPUTATIONAL AFFECTIVE KNOWLEDGE REPRESENTATION}

Table 3.4: $\mu_{A^{i}}(u)$ values for the example $(u=0.625)$.

\begin{tabular}{ccccc}
\hline & Neutral & Weak & Medium & Strong \\
\hline$\mu_{A^{i}}(0.625)$ & 0 & 0 & 1 & 0 \\
\hline
\end{tabular}

Table 3.5: Example of $\mu_{B^{j}}(\alpha)$ values for $\alpha=245^{\circ}$. For the Portuguese and Swedish labels see Table 3.1.

\begin{tabular}{lrrrrrrrrrr}
\hline & Fear & Surprise & Disgust & Anger & Boredom & Sleepiness & Sadness & Excitement & Calm & Happiness \\
\hline Portuguese & 0.075 & $\approx 0$ & 0.374 & 0.037 & 0.998 & 0.723 & 0.055 & $\approx 0$ & 0.253 & $\approx 0$ \\
Swedish & $\approx 0$ & $\approx 0$ & 0.059 & $\approx 0$ & 0.311 & 0.844 & 0.147 & $\approx 0$ & 0.079 & $\approx 0$ \\
Spanish & $\approx 0$ & $\approx 0$ & 0.271 & $\approx 0$ & 0.999 & 0.479 & 0.087 & $\approx 0$ & 0.102 & $\approx 0$ \\
\hline
\end{tabular}

a Medium intensity in Portuguese, Swedish, and Spanish. The formulas to obtain these values are described in detail in [220].

The Emotion Expression process is a fuzzification process that allows an affective agent to express an affective state using the same label/word in the same language as its human interlocutor. We provide two fuzzy functions, $\mu_{A^{i}}(u)$ and $\mu_{B^{j}}(\alpha)$ (described in detail in [220]), to estimate the most appropriate terms to express the intensity and type of emotion, respectively, according to the cultural environment. To illustrate how these fuzzy functions work, let us consider the following example in which an affective agent wants to express its affective state represented by the vector $\overrightarrow{v_{e}}$ (see Figure 3.8), formed by the components Pleasure $=-0.264$ and Arousal $=-0.566$. The angle of the vector is $245^{\circ}$ and the modulus of the vector is 0.625 . According to the modulus of the vector, the formula for the intensity of the Emotion Expression process produces the results shown in Table 3.4. Therefore, the intensity level of the emotion is Medium. Table 3.5 shows the estimated probability of the possible emotion labels for the Portuguese, Swedish, and Spanish languages applying the formula for the type of the Emotion Expression process to a direction of the vector of $245^{\circ}$. According to this results, we can conclude that the most probable emotion label to be used by the agent for the Portuguese and Spanish languages is "Boredom" (i.e., "Aborrecimento" for Portuguese and "Aburrimiento" for Spanish) with a probability of 0.998 and 0.999 respectively, while for Swedish it is "Sleepiness" (i.e., "Trött") with a probability of 0.844 . 


\subsection{DISCUSSION}

Table 3.6: Results of the chi-square test for the study of the influence of gender and age on the assignment of pleasure and arousal values for the ten emotions in the Portuguese and Swedish samples. Note that * indicates p-value $<0.05$.

\begin{tabular}{|c|c|c|c|c|c|c|c|c|}
\hline \multirow[b]{3}{*}{ Emotion } & \multicolumn{4}{|c|}{ Portuguese } & \multicolumn{4}{|c|}{ Swedish } \\
\hline & \multicolumn{2}{|c|}{ Gender } & \multicolumn{2}{|l|}{ Age } & \multicolumn{2}{|c|}{ Gender } & \multicolumn{2}{|l|}{ Age } \\
\hline & $\chi^{2}$ value $(\mathrm{df})$ & p-value & $\chi^{2}$ value (df) & $\mathrm{p}$-value & $\chi^{2}$ value $(\mathrm{df})$ & $\mathrm{p}$-value & $\chi^{2}$ value $(\mathrm{df})$ & p-value \\
\hline Fear & $11.084(14)$ & 0.679 & $12.471(14)$ & 0.568 & $6.789(8)$ & 0.559 & $5.618(8)$ & 0.056 \\
\hline Surprise & $9.055(8)$ & 0.337 & $18.130(8)$ & $0.020^{*}$ & $5.618(6)$ & 0.467 & $16.206(6)$ & 0.766 \\
\hline Disgust & $18.528(14)$ & 0.183 & $12.134(14)$ & 0.595 & $16.206(13)$ & 0.238 & $3.520(13)$ & 0.382 \\
\hline Anger & $15.896(14)$ & 0.319 & $18.255(14)$ & 0.195 & $3.520(4)$ & 0.474 & $1.346(4)$ & 0.133 \\
\hline Boredom & $8.443(7)$ & 0.295 & $4.610(7)$ & 0.707 & $1.346(4)$ & 0.853 & $10.313(4)$ & 0.130 \\
\hline Sleepiness & $9.380(6)$ & 0.153 & $9.619(6)$ & 0.141 & $10.313(6)$ & 0.112 & $10.059(6)$ & 0.092 \\
\hline Sadness & $6.808(6)$ & 0.338 & $9.303(6)$ & 0.157 & $10.059(8)$ & 0.260 & $11.437(8)$ & $0.043^{*}$ \\
\hline Excitement & $1.656(3)$ & 0.646 & $5.300(3)$ & 0.151 & $11.437(9)$ & 0.246 & $10.237(9)$ & 0.559 \\
\hline Calm & $3.954(9)$ & 0.914 & $15.151(9)$ & 0.086 & $10.237(14)$ & 0.744 & $6.179(14)$ & 0.152 \\
\hline Happiness & $2.893(4)$ & 0.575 & $3.502(4)$ & 0.477 & $6.179(7)$ & 0.518 & $0.518(7)$ & 0.054 \\
\hline
\end{tabular}

\section{Gender and age comparison}

As in the case of language, we performed a comparative analysis of the samples to identify the existence of possible discrepancies between gender and age when assessing the levels of pleasure and arousal for the ten emotions using Chi-square test. Applying a criterion of homogeneity for sample size, we divided the age into two groups according to the median, which was 29 for the Swedish participants and 32 for the Portuguese participants.

Table 3.6 shows the results of the comparative analysis of the samples for gender and age using the Chi-square test. The starting hypotheses for this analysis are: the null hypothesis $h_{0}$ "the angle associated with an emotion is independent of gender/age"; and the alternative hypothesis $h_{1}$ "the angle associated with an emotion and the gender/age have some degree of dependence". Regarding gender, we can see that in the experiment conducted with both Portuguese and Swedish participants, the p-value of the results indicate that there is not enough evidence to reject the null hypothesis $h_{0}$ at a confidence level of $95 \%$. Therefore, there is not enough evidence to determine that there is a dependence between gender and the angle that participants associated with emotions.

Similarly, attending to the general results for the age comparison, we can 


\section{CHAPTER 3. COMPUTATIONAL AFFECTIVE KNOWLEDGE REPRESENTATION}

observe that in both languages there is not enough evidence to indicate that there is a dependence between age and the angle associated with emotions. However, we find two emotions, surprise in the case of Portuguese and sadness in the case of Swedish, where the p-value is less than 0.05. That is, with a confidence level of $95 \%$, the null hypothesis $h_{0}$ is rejected. In other words, there seems to be a dependence between the selected angle and age for these two emotions. This result may be due to a bias in age that occurs when dividing the samples into two homogeneous groups comprising different age ranges.

\section{Importance of multidimensional models}

We can clearly observe, by the results of this study, that emotions, defined by the dimensions of pleasure and arousal, do not mean the same thing everywhere. To design universal affective agents, we need multidimensional models that capture these cultural differences. People from different parts of the world can physically and psychologically feel emotions in a similar way, but perceive, interpret, or convey the emotions differently. Such differences in perception of emotions can contribute to what attitude people have toward certain emotions and emotional expressions. Affective agents must be able to represent, detect, and express emotions in a way that follows the individual's conceptions. Natural language processing (NLP) has mainly been based on supervised learning, trained on large sets of conversational data, where deep learning methods have seen great potential. In the context of emotion detection NLP is usually based on finding specific predefined keywords as "Surprised", "Sad", and "Angry" [84]. The observed cultural differences in the interpretation of emotions can thus be miss represented in these models. The multidimensional model presented in this study can provide affective agents with enhanced capability of emotional differentiation, which is increasingly important in phase with the progressed development of NLP-based conversational interfaces. 


\subsection{APPLICATIONS}

\subsection{Applications}

\section{A cross-cultural affective agent model}

Although the representation based on the dimensions of pleasure and arousal can be considered universal in the definition of the agent's internal structure of knowledge representation, for an affective agent to be able to represent, interpret, and express affective states it is necessary to adapt this representation to the cultural environment in which it is going to be used. In addition, when an affective agent evolves in several different cultural environments, it also requires the ability to adapt its affective behavior to these environments. This can be achieved by defining a specific pleasure and arousal space for each cultural environment. In this paper, we present three different models of affect representation adapted to Portuguese, Swedish, and Spanish. All these two-dimensional representations can be easily incorporated into the affective component of a multicultural affective agent allowing it to interpret, represent, and express affective states using the same language as its human interlocutor and adapting to his/her cultural variations. Then, through the Emotion Representation process and the Emotion Expression process, the agent can represent and express different affective states in different languages. The example presented in Section 3.6 illustrates how an affective agent could use these models to adapt its behavior to the cultural environment of its human interlocutor.

\section{Application in Human-Aware Planning}

The multicultural representation model of affect proposed in this paper can be utilized in a human-aware planning scenario [41], where a software agent requires a model of a human user's emotional state for planning its actions in interaction with the user.

Let us consider a software assistant, designed to manage a human user's stress during their interaction by adjusting its behavior or output. The agent utilizes a logic-based knowledge base that captures: i) a model for representing emotions, ii) a human stress-model, in which different sets of emotions 


\section{CHAPTER 3. COMPUTATIONAL AFFECTIVE KNOWLEDGE}

REPRESENTATION

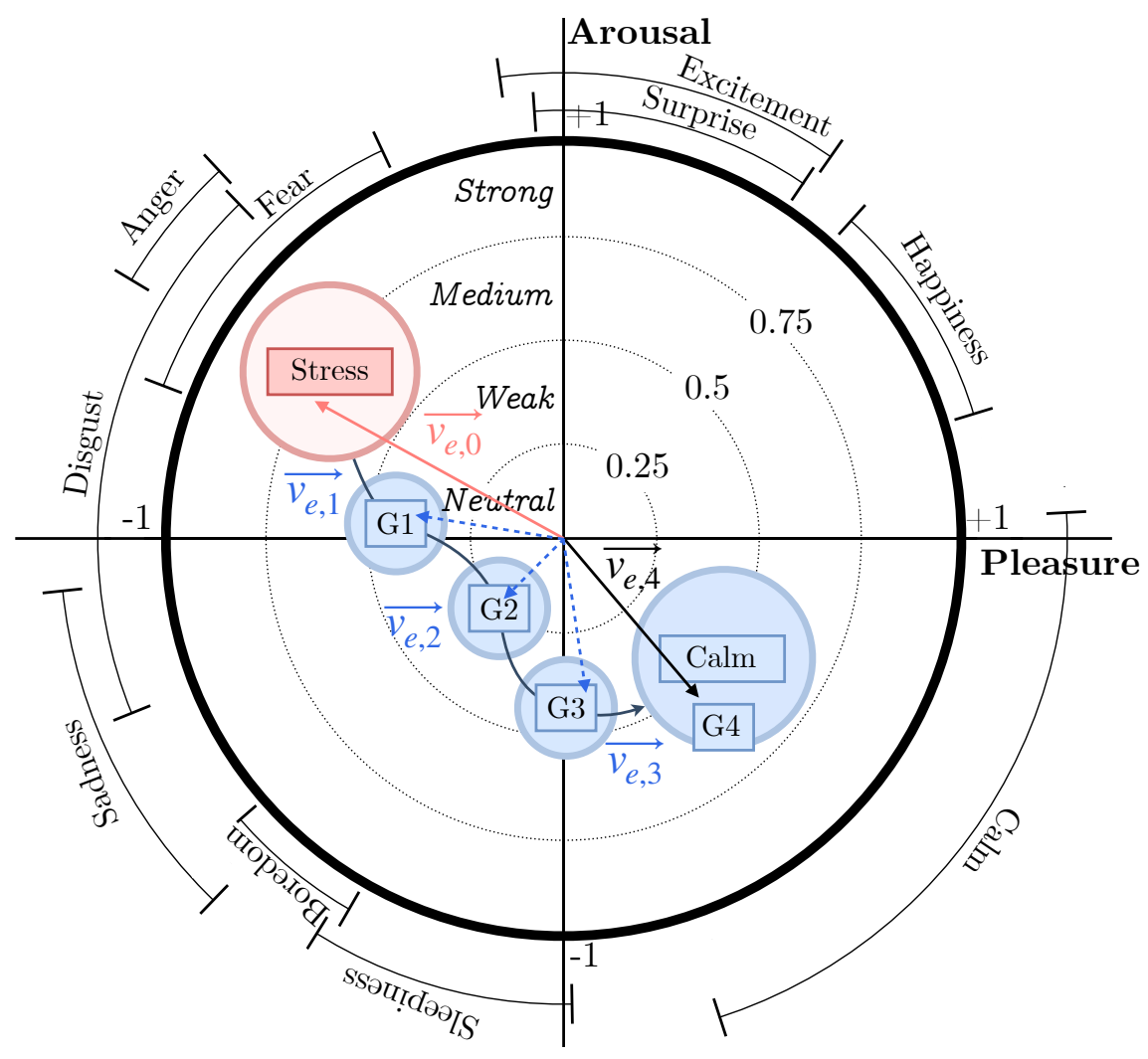

Figure 3.10: A stress relief plan consists of a sequence of subgoals (G1-G3) and a final goal (G4).

are linked to stress-states, and iii) a decision-model, through which the agent generates a sequence of actions aiming to change a set of unwanted emotions to a set of wanted emotions. The agent does this by influencing the user's pleasure and arousal through appropriate interactive actions. In order to identify goals (suitable emotional change) and avoid obstacles (less suitable emotional change), the agent deliberates about alternative plans by considering emotions defined in the pleasure-arousal space (see Figure 3.10).

Stress-model: Previous research has observed that psychological stressresponses often include a complex array of "negative" emotions, such as depression, anxiety, anger, and distress [63]. Following these results, we can define negative stress as a set of emotions, which share a high level of arousal and (usually) low pleasure. This would mean that the upper-left part of the pleasure-arousal space captures a set of emotions that represent negative 


\subsection{APPLICATIONS}

stress. A desired goal of the agent is to decrease stress. A stress-relief state can (intuitively) be defined by a set of emotions with lower arousal and/or higher pleasure. However, there may be more or less suitable ways for achieving stress relief. Whether to decrease arousal or increase pleasure in a moment depends on the current emotional state of the human, the current context, and a variety of personal and cognitive attributes. Desirable plans for stress relief are generated by the system's decision-model.

Decision-model: A method for defining a decision-model for picking suitable actions to influence emotions is to conduct knowledge elicitation processes [208] with relevant experts, such as psychologists. By consulting experts (as well as relevant psychological theories) we can understand how to appropriately deal with emotional change, and capture this knowledge in explicit decision-rules. These rules helps the agent to anticipate how the mental model of the human will change and pick suitable actions in each state of the interaction. A decision-model can be modelled in the structure of a transition system [83] (consisting of states $S$ and transitions between states), in which each state corresponds to a set of emotions, and transitions correspond to suitable change, triggered by an action or a set of actions, between emotional states. A sequence of actions that trigger transitions between states constitute a plan to reach emotions that represent stress relief (e.g., from a state representing "Stress", a set of actions and transitions lead to a state representing "Calm"). Through the constraints of the transition system, the agent can decide which emotions to aim for next, depending on the current emotional state. The decision-model can, for instance, be implemented in Action Reasoning [83]. In action reasoning, a set of (emotion influencing) actions $A$ are defined. An action $a \in A$ has preconditions, stating from which emotional states an action can be executed, and post-conditions, stating the estimated emotional change (proceeding state) after the action is executed. The solutions of the planning problem (the generated plans) are returned as trajectories of length $\mathrm{n},\left\langle s_{0}, A_{1}, s_{1}, A_{2}, \ldots, A_{n}, s_{n}\right\rangle$, which are sequences of sets of actions $\subseteq A$ and states $s_{n}$ of $S$ satisfying the conditions for suitable emotional change specified by the decision-model.

Interaction: The actions of $A$ are by extension connected to specific interactive actions by the agent, depending on how it is embodied/visual- 


\section{CHAPTER 3. COMPUTATIONAL AFFECTIVE KNOWLEDGE REPRESENTATION}

ized and on the particular application, e.g., changing its behavior, facial expressions, voice feedback, or by other means influencing the human's beliefs, indirectly changing pleasure and arousal. For instance, interactions could result in the following trajectory, consisting of emotional states and sets of actions: $\left\langle s_{0}:\{\right.$ Fear, Anger $\}, A_{1}:\{$ Pause, Speak slowly,Clarify expectations $\}$, $s_{1}:\{$ Nervousness $\}, A_{2}:\{$ Smile, Show instructions $\}, s_{2}:$ Collected $\}$, $A_{3}:\{$ Pause, Smile, Kind voice $\}, s_{3}:\{$ Calm $\left.\}\right\rangle$.

The interaction is dependent on reliable ways to elicit pleasure and arousal levels of the human user. Depending on the application, this can for instance be achieved by using appropriate machine learning models for classifying pleasure and arousal from psychological data, and/or through a user interface, where the human user supplies the system with subjective emotional data in terms of emotion-labels (in the user's own language).

\subsection{Conclusions}

One of the main challenges in improving human-machine interaction and simulations of human behavior and human social organizations is to establish models that allow agents to represent, express, and interpret affective states. In this sense, dimensional representations of affect seem to be promising since they allow modeling affective states through continuous dimensions. However, it is necessary to consider that, in dimensional approaches, cultural and language variations in the values of the dimensions associated with the different affective states arise. Therefore, it is necessary to establish representation models of affect capable of adapting to these cultural variations. In this paper, we show the results of experiments conducted to adapt a model of affect representation to the Portuguese and Swedish language. For this purpose, we apply a methodology based on two experiments. In the first experiment, we establish the most feasible translations for the affective categories that define the dimensions of pleasure and arousal. The obtained results show the importance of performing this type of experiments when translating complex cognitive constructs such as emotions or affective categories. For example, in the case of Swedish, the most common translation for the pleasure dimen- 


\subsection{CONCLUSIONS}

sion is "Behag". However, participants preferred to choose the words "Lycka" and "Glädje" while, for misery, participants preferred "Otillfredsställelse" and "Missnöje" over "Obehag" which is the most common translation. The same pattern was observed in Portuguese, where the most common translations in the literature for the pleasure dimension are "Prazer" and "Desprazer". However, participants preferred to choose "Felicidade" and "Alegria" for pleasure and "Infelicidade" and "Sofrimento" for misery.

Based on the results of the first experiment, in the second experiment we explored the regions associated with the different emotions in the pleasurearousal space. To avoid bias, we decided to contextualize each affective category with the words most rated by participants. The analysis of the results of this second experiment shows the importance of adapting the models of affect representation to the cultures and languages in which they will be used. We have shown the existence of significant differences in the values of pleasure and arousal associated with emotions across languages and cultures. These differences increase when the languages come from different language families, as in the case of Portuguese and Swedish. The results also show that there do not appear to be significant differences in gender or age when associating levels of pleasure and arousal with emotion labels.

The results of the second experiment have been used to design two new computational models of affect representation based on the dimensions of pleasure and arousal adapted to Portuguese and Swedish languages. In both models affective states are represented as vectors in which the direction indicates the type of affective state and the modulus indicates its intensity. To determine the type of affective state we use a representation based on probability regions. Probability regions allow us to estimate, with a certain degree of uncertainty, the probability that a vector belongs to a certain type of affective state.

We propose two applications for these models. On the one hand, we propose a model of affective agent capable of evolving in different cultural environments. The affective agent incorporates multiple models of affect representation adapted to different cultural environments allowing it to adapt its affective behavior according to the language and culture of its human interlocutor. On the other hand, we propose the use of these models in a 


\section{CHAPTER 3. COMPUTATIONAL AFFECTIVE KNOWLEDGE}

REPRESENTATION

human-aware planning scenario. The agent represents the affective state of the user as a function of his cultural environment. Then, the agent uses the vector representation to guide its actions in order to influence the user's affective state and redirect it towards a target affective state.

The multidimensional model presented in this study can provide affective agents with enhanced capability of emotional differentiation, which is increasingly important in phase with the advances of NLP-based conversational interfaces. The proposed model can enable affective agents to represent, detect, and express emotions in a way that follows an individual's conceptions, independent on cultural context.

We are currently working on the development of the application models proposed in this paper. We are also interested in studying the influence of other dimensions, such as the dominance dimension, on the model of affect representation. In particular, we are interested in analyzing whether these dimensions allow us to improve the identification of affective states such as "Fear" or "Anger". 



\title{
Chapter 4
}

\section{A fuzzy appraisal model for affective agents adapted to cultural environments using the Pleasure and Arousal dimensions}

\author{
Joaquin Taverner, Emilio Vivancos, and Vicente Botti \\ Information Sciences, 546:74-86, 2021 \\ Doi: https://doi.org/10.1016/j.ins.2020.08.006
}

\begin{abstract}
Humans use rather vague and imprecise words to express emotions. Therefore, fuzzy logic allows computational affective models to use emotions in the same way that human beings express them. However, people from different cultures and languages assign different meanings to the same emotion word. Unfortunately, there are still no affective computing models that really take these two factors into consideration. In this paper, we propose a fuzzy model of appraisal for multiagent systems that is adapted to Spanish-speakers. Our methodology has two steps. First, the agent evaluates an event using a set of fuzzy appraisal rules that returns a fuzzy emotion. Then, a defuzzification process returns the Pleasure and Arousal dimensions of the emotion that will be internally represented as a vector in a twodimensional space. This vector is used to update the agent's mood according to the agent's personality. The agent can express this internal emotional state using a fuzzification process that translates the agent's mood into a fuzzy emotion. This fuzzification process uses the results of an experiment to generate a fuzzy emotion that is adapted to the cultural environment in which the agent is located. This methodology can be easily adapted to other languages.
\end{abstract}




\subsection{Introduction}

Over the last few years, the way in which humans interact with machines has been changing. The emergence of virtual assistants for both mobile phones and home is helping to facilitate how humans communicate with machines. However, human beings are emotional beings and these interactions can be improved by taking into account human emotions. Emotions affect the way in which we interact with others and establish links that will be the basis of future relationships. Thus, a computer system that can express emotions and understand the emotions of its human interlocutor will be able to improve its interactions with humans.

Affective computing [170] is the area of computation related to emotions that is based on theories of the sciences of psychology and cognition. One of the main goals of affective computing is to design computational models of affect to simulate human emotional behavior in a realistic way. Thus, different proposals have been designed from the computational [81, 148, 186] and psychological [201] perspectives. However, in general, most of these proposals are simplifications of psychological theories that were not originally proposed to be incorporated into computational models, thus reducing emotions to simple labels (as in emotion recognition) [103]. When this label-based representation is used, some inherent properties of emotions, such as the intensity or the proximity to other emotions, is lost. In this paper, we propose an internal representation of emotions using a continuous multidimensional space that is more appropriate for use in computational models since it provides a great capacity to represent emotions and mood, and we analyze their variations when receiving both internal and external stimuli.

Considering that humans use expressions like "very happy" or "a little sad", in recent years, some authors have proposed the use of fuzzy logic [238] to model affective processes since fuzzy logic is closer to the way in which human beings express their emotions [116]. On the other hand, another important difficulty when studying emotions is that the internal way of feeling emotions is highly dependent on the person's personality and the cultural environment. Different authors have shown that emotions depend on language and culture [196, 227]; specifically, the same emotion name/label can 
be interpreted in a different way in different cultures. Moreover, different people show different amounts of spoken affect according to their language and cultural characteristics. That means that any appraisal process used in an affective agent should be adapted to the culture and language in which the agent will be used. Therefore, when computational models of affect are designed, the cultural environment in which they will be used must be considered. However, even though cultural and language factors are taken into account in other domains such as personal assistants, to our knowledge, there are still no affective computing models that really take cultural and language factors into consideration.

This paper presents a new appraisal model for a multi-agent affective BDI architecture [180] based on fuzzy logic that is adapted to cultural and language factors. Our proposal is composed of two independent processes. First, in the Event Appraisal process, an event is evaluated using fuzzy rules, producing a fuzzy emotion as a result. Second, the Affect Adaptation process defuzzificates the appraised emotion obtaining its Pleasure and Arousal dimensions, which are adapted to the cultural and language in which the emotion was elicited. These dimensions are used to update the agent's mood using the elicited emotion, taking into account the agent's personality and the language and culture in which the agent is located.

\subsection{Previous work}

Over the years, different theories have been developed to explain what emotions are and how emotions are elicited. Appraisal theories postulate that emotions are the result of an evaluation process that is triggered when an event occurs. From a theoretical perspective, the appraisal process is a response to a stimulus that triggers a series of processes that is in charge of evaluating that stimulus and, as a result, eliciting an emotion. In most cases, the appraisal process is explained using a set of variables known as appraisal variables. The number of emotions that can be generated in each appraisal theory depends on the number of appraisal variables used and the number of values that these variables can have. This way of eliciting emotions as the re- 


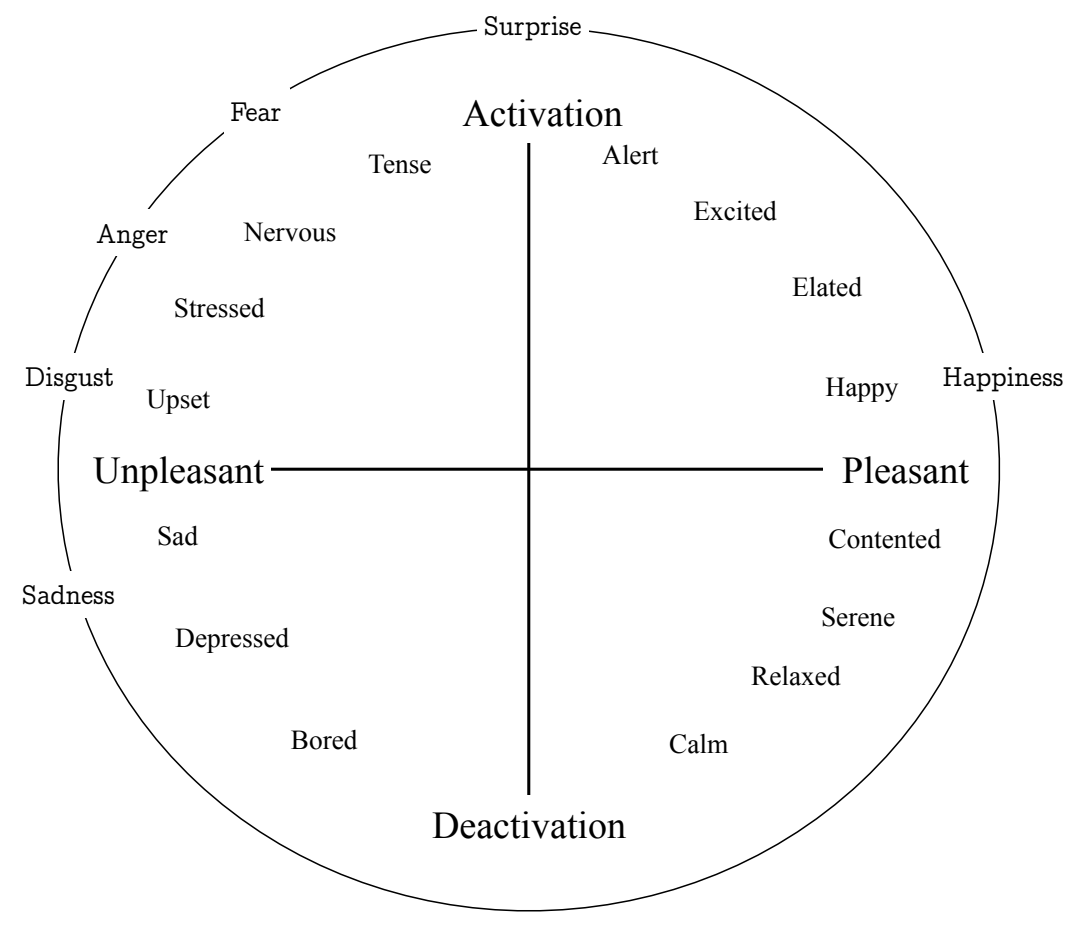

Figure 4.1: The circumplex model of affect: the outer ring contains the "prototypical emotion episodes" that match the basic emotions of Ekman's theory. Source: [195].

sult of the evaluation of a set of variables is very useful when using this model in a computer system. This is why, in recent years, most affective intelligent agent models have incorporated an appraisal process [6, 81, 148].

Different authors use different sets of appraisal variables to define the appraisal process. For example, Frijda [72] posits that the familiarity, expectedness, valence, controllability, agency, certainty, and importance of the detected event should be taken into consideration. The Scherer approach [201] makes a more detailed description of the factors involved in the appraisal processes such as predictability, urgency, power, or suddenness. Scherer also proposes a series of patterns that relate the appraisal variables to different emotions in order to propose an expert system for affective computing [200]. However, Scherer uses twenty-two appraisal variables, which makes this model 


\section{CHAPTER 4. A FUZZY APPRAISAL MODEL FOR AFFECTIVE AGENTS (SELECTED PAPER)}

very complex to use. This is why this model has not been widely used in affective computing. In contrast, the OCC (Ortony, Clore, and Collin) model [163], which uses only eight appraisal variables, is the most commonly used model in affective computing. On the other hand, basic emotion theories, like the one proposed by Ekman [65], hold that there is a limited number of emotions (Happiness, Surprise, Fear, Anger, Disgust, and Sadness) and that each detected event elicits an associated emotion. According to this theory, these emotions are universally understood in different cultures and languages.

While Ekman argues that basic emotions are universal and transcultural, constructivist theorists, like Russell, argue that emotions depend on language and culture [196]. Russell's theory [195] is based on the fact that there is not always a direct correspondence between the words representing emotions in two different languages. For example, in the German language, there is an emotion called "Sehnsucht" [107], whose meaning is "a strong desire for an alternative life". In languages such as English or Spanish, there is no single word to express this emotion. Russell's theory is more consistent with findings in cognitive neuroscience [174]. These claim that emotions are a combination of a small number of dimensions rather than being directly related to events, as in Ekman's theory and other basic emotion theories [65], which affirm that emotions are related to an independent neuronal system. Russell relates emotions with a pair of values, Pleasure and Arousal, providing a two-dimensional space for the representation of emotions. In addition, through experimentation, he observed that emotions follow a circular pattern in these two dimensions, which led him to propose his best-known theory: The Circumplex Model Of Affect [193] (Fig. 4.1). Mehrabian proposed the PAD (Pleasure, Arousal, Dominance) model [154], which adds the Dominance dimension to represent emotions. This third dimension can help to clarify negative emotions. For example, the emotion of Fear is associated with a low level of Dominance, while the emotion of Anger is associated with a high level of Dominance. However, the Dominance dimension is not included within many models, and it is used as an appraisal variable instead [147]. Moreover, this dimension does not seem to have a high variability among emotions [107], and some authors have found that human beings have difficulty and show confusion when they try to assign a Dominance value to the emotions that they are feeling [122]. 


\subsection{PREVIOUS WORK}

Other authors, such as Reisenzein [183], showed the relationship of the intensity of emotions with the variables of Pleasure and Arousal. In an experiment, Reisenzein observed that the levels of Pleasure and Arousal are related to the intensity of emotions. Apparently, the intensity of emotions is proportional to the levels of Pleasure and Arousal so that the higher these levels, the greater the intensity. For example, the intensity of the Happiness emotion is highly dependent on the level of Pleasure, while the intensity of the Alert emotion is more dependent on the level of Arousal. He also observed that certain minimum levels of Pleasure and Arousal are required in order for emotions to show themselves. Therefore, when both variables have low values, the absence of emotions can be considered.

\section{The relation between emotions, personality, and mood}

When modeling the appraisal process, the relation between emotions and other affective characteristics such as personality or mood must be taken into consideration. Although the difference between emotions and mood continues to be a topic of debate [19], in general, it is accepted that an emotion is a rapid response to a given stimulus, while mood has a longer duration (from minutes to days) and a lower intensity than emotions and is not related to any particular stimulus. Instead, mood is produced by a succession of stimuli and other factors such as the context or the person's personality [64]. Empirical evidence suggests that there is a critical impact of emotions on cognition and a high variability of this impact among individuals with different personality factors. Personality can make a person more or less likely to experience certain types of mood [133].

One of the most commonly used models to define personality is the fivefactor model (FFM) [152] in which personality is defined using five variables (or traits): Openness, Conscientiousness, Extraversion, Agreeableness, and Neuroticism. Different works that are based on the FFM relate personality factors with cognitive abilities, behaviors, and social skills. For example, there is evidence [56] that the Extraversion factor predisposes one to experience a more positive affect more frequently and with greater intensity, appraising emotions such as joy, enthusiasm, or energy. In contrast, Neuroticism pre- 
disposes one to experience a negative affect as well as suffering from negative moods such as fear, sadness, depression, or anger.

\section{Emotions in affective agents}

In recent years, different models have been proposed to simulate emotions using intelligent agents $[5,47,186]$. The proposed model for the EMA affective agent [148] is based on the subsequent formalization of the theory presented by Smith and Lazarus [135] that was carried out by Gratch and Marsella [88]. They propose a one-level appraisal process that takes into account past, current, and future events. They define different thresholds for the appraisal variables to elicit emotions [88]. In this model, intensity is defined as the product of different numeric appraisal variables. For example, the product of desirability and likelihood is used to estimate the intensity of the Joy emotion. The appraisal process of EMA has been used as a base to define emotion elicitation processes in other affective agent models such as GenIA ${ }^{3}$ [6].

Another interesting approach is the one proposed by Gebhard in ALMA (A Layered Model of Affect) [81]. This model is based on the OCC appraisal theory [163] and models appraisal using subjective appraisal rules; therefore, each agent can have different appraisal rules. In these rules, the different appraisal variables are bounded by numeric values for eliciting each emotion. The authors also propose a decay rate for emotions, which decreases the intensity of the emotion over time until the emotion disappears.

Generally, humans express emotions and moods through terms such as "very happy" or "a little happy". Therefore, in the last few years, some authors have proposed using fuzzy logic to define affective computational models. For example, the appraisal model for FLAME [67], which is based on the Roseman [190] and OCC theories, uses variables with fuzzy values. In this approach, each emotion is selected based on a series of simple rules. Another interesting approach is the one presented by Jain et al. in EMIA [108]. The authors propose a fuzzy logic emotional model for five of Ekman's basic emotions (all except the Disgust emotion) [65]. The EMIA model implements a simplification of the Scherer model using five appraisal variables. 


\subsection{PREVIOUS WORK}

The appraisal process is affected by the agent's memory and allows more than one emotion to be elicited in each appraisal cycle. All of the emotions have a fuzzy intensity value, and this intensity is modified over time by an emotion decay process.

Other affective characteristics, such as personality or mood, have also been used in affective computing to define the emotion appraisal process. Personality is usually used to create behaviors in multi-agent systems [39, 215]. For example, in [38], the authors propose that personality and mood have an effect on the appraisal variables and thus on the elicited emotions. This model proposes that personality affects the ranges of the fuzzy appraisal variables. Similarly, in [39], a model that uses fuzzy appraisal variables is proposed based on the Integrative Model [189]. That model is designed to adapt the cognitive and affective capacities of an agent to internal and external factors among which gender or personality are considered. To this end, each appraisal variable is defined with a membership function that is adapted according to these internal and external factors. The modification of the membership function of each appraisal variable is done both by modifying the type of function (e.g., pyramidal, trapezoidal) and by modifying the parameters of the membership function. However, at the moment, this model is only a proof of concept.

\section{Discussion}

When affective agents are modeled to simulate emotional behavior, the most important task is to choose the right emotion when an event is triggered. This is because the generated emotion will affect the cognitive processes of the agent, such as reasoning or the selection of behavior. The selected emotion will also have an impact on affective characteristics such as mood or empathy. Therefore, generating an incorrect emotion when an event occurs can cause erroneous behaviors that decrease the realism of the emotional behavior simulation. To model this process, several variables must be taken into account, such as the agent's concerns or the desirability of the event and other factors such as personality or mood. Several affective models use numerical variables, but this is problematic because it is somewhat different from how 


\section{CHAPTER 4. A FUZZY APPRAISAL MODEL FOR AFFECTIVE AGENTS (SELECTED PAPER)}

human beings express their emotions; humans find it very difficult to quantify emotions with numerical values.

In our model, we have decided to use fuzzy logic as a more natural way of eliciting emotions in the appraisal process. Models that use fuzzy logic are more suitable for simulating the human appraisal process due to the "fuzzy" way of expressing emotions by human beings. The use of fuzzy logic helps the understanding of the emotional states of the human beings that are interacting with the agents. In recent years, some proposals for emotion elicitation processes have been defined using fuzzy logic. For example, FLAME [67] proposes a small set of rules but involves the user too much in its implementation. In addition, even though the FLAME model allows emotions with intensity to be generated, the simplicity of the rules makes it a very basic model. For example, the Joy emotion is elicited if a desirable event occurs without taking into account the rest of the appraisal variables. Therefore, the rules are too simple, assigning the selected emotion by using only one appraisal variable. In addition, the appraisal variables are evaluated in a binary way: desirable or undesirable. Therefore, there are only a few combinations of values available to generate different emotions. Thus, this model has a very limited catalog of emotions, which, as stated above, hinders the user experience. Other fuzzy models, such as the model presented in EMIA [108], proposes a fuzzy logic approach for the appraisal process. That model reduces Scherer's model to five appraisal variables. However, the final model has two hundred rules to define an appraisal process that generates only five emotions. Therefore, even though the FLAME model is very basic due to the small number of rules, the EMIA model is too complex given its enormous number of rules. In addition, the EMIA model does not consider mood or personality in the appraisal process.

When simulating human affective behavior, the effect that different external and internal factors (e.g., personality or mood) have on cognitive processes must be taken into account. These factors have a direct consequence when evaluating an event, biasing the appraisal processes and consequently influencing emotions or mood. Some current proposals, such as the one presented in [38], use personality and mood to influence emotion selection in the appraisal process. However, in this model, the intensity of emotion is not 


\subsection{PREVIOUS WORK}

contemplated. Therefore, this model becomes much simpler to design and implement. This can have a negative effect on the behavior of the agent since the catalog of emotional responses decreases. Other proposals such as the one proposed in [39] take into account the importance of these internal and external factors, but that model has only been tested with the gender factor. Furthermore, although it is accepted that factors such as personality have an effect on emotions, which predisposes the individual to certain types of emotion, there is not enough evidence to determine what the effect of personality is on an appraisal variable.

As we have stated above, there is a large number of appraisal theories that try to explain how emotions are generated. Each theory has a series of advantages and disadvantages. For example, the patterns that Scherer [200] proposes in his computing model of affect are interesting when determining which variables affect each emotion. However, Scherer uses a large number of variables, which makes this model very complex for development in an affective agent. Furthermore, in this model, the intensity of the emotions is not considered. This may affect the behavior of the agent since the catalog of actions of the agent will be very limited because the intensity of the emotion cannot be taken into account to decide the action to be executed. A small catalog of emotions can affect the interactions with human users because, if an agent shows the same emotions very often, the agent loses credibility and the users tend to become bored. Therefore, the more we expand the catalog of emotions of an agent, the greater the number of different emotional behaviors that can be simulated.

The internal representation of emotions must also be taken into account when defining an emotional model for an affective agent. Most of the emotional models proposed to date reduce the representation of emotions to simple labels [69]. However, dimensional representations seem to be more appropriate for developing computational models since they allow emotions to be stored using variables instead of simple labels and variables can hold a wider range of values. The Circumplex Model of Affect [193] (Fig. 4.1) has already been used in affective computing [198]. Nevertheless, the schema proposed by Russell is only a reinforcement for his theory. In this scheme, he uses a simplified spacial location for each emotion, but this is unnatural, and, conse- 
quently, an agent using this simplified representation of emotions could show erratic behavior that is very different from the real behavior of a human being. Therefore, performing a direct use of this model in a computational system could lead to unreal emotional behaviors.

In the next section, we introduce our new fuzzy appraisal model based on representing emotions in a Pleasure-Arousal space. Section 4.3 describes how a fuzzy rule-based system selects one or more emotions by evaluating the appraisal variables. Section 4.3 presents the Pleasure-Arousal space where agents can internally represent the appraised emotions using a defuzzification function. This internal representation of emotions allows the agent to update its mood as presented in Section 4.3. Finally, when the agent needs to communicate or express its internal emotional state, a fuzzification process (presented in Section 4.3) translates the internal emotional state represented in the Pleasure-Arousal space into a fuzzy emotion. The fuzzification process uses the results of an experiment to generate a fuzzy emotion that is adapted to the language and cultural environment of the human interlocutor of the agent. The article ends with conclusions and future works.

\subsection{A model for emotion elicitation in affective agents}

The appraisal process is one of the most important processes in an affective agent because suitable agent behavior depends on the emotion selected. A good definition of this process is even more important when the agent is designed to interact with humans since erratic or erroneous behavior of the agent can make the human become frustrated. In addition, processes such as the selection of the action to be executed or the updating of the agent's mood will depend on the emotion elicited.

As we have discussed above, there are different psychological theories to explain emotional processes. Appraisal theories provide an explanation of how an emotion is generated when an event is perceived. Alternatively, other theories, such as Russell's theory [193], offer the possibility of relating emotions with dimensional variables. We propose a fuzzy model of emotions that 


\subsection{A MODEL FOR EMOTION ELICITATION IN AFFECTIVE AGENTS}

adapts the advantages of the appraisal models for selecting an emotion when an event occurs and the advantages of representing the emotion in a multidimensional space. As a novelty, by using this multidimensional representation, our model can adapt emotions to the agent's affective characteristics and to the culture and the language in which the agent will be located.

Our model is composed of two processes. First, in the Event Appraisal process, an event is evaluated by our fuzzy appraisal algorithm and produces an emotion which we will refer to as Appraised Emotion. This fuzzy process allows us to generate emotions and their intensity, capturing the kind of uncertainty that humans beings use when expressing their emotions. Second, in the Affect Adaptation process, the mood is updated based on the Appraised Emotion, the agent's personality, and the culture and language in which the agent is located. The following section describes both processes in detail.

\section{The Event Appraisal process}

The Event Appraisal process defines a fuzzy appraisal process to select an emotion when the agent perceives an event. The selected emotion corresponds with one of the six basic emotions proposed by Ekman [65]: happiness, disgust, sadness, anger, fear, and surprise. Moreover, based on the emotions proposed by Russell in [196], two emotions, calm and boredom, have also been incorporated to represent the lack of interactions between the agent and the user and also the lack of relevance of the event.

We have designed a fuzzy rule-based system [122] that, depending on the value of the appraisal variables, returns the elicited emotion and its intensity. The fuzzy values used to define the rules are based on the computational model proposed by Scherer [200], but we solve the main weakness of this model: Our model needs a smaller number of appraisal variables and it also calculates the intensity of the elicited emotion. Moreover, in our model, the values of each appraisal variable are associated with the intensity of the emotions as proposed by $\mathrm{OCC}^{1}$. According to this theory, two of the most relevant appraisal variables attributed to emotions produced by events, such as hap-

${ }^{1}$ The relationship between the appraisal variables of both the OCC and the Scherer model can be found in $[200]$ 
piness or sadness, are desirability and likelihood. Therefore, in our model, we associate these two variables with Scherer's model to obtain the label and the intensity of the emotions using fuzzy variables. The appraisal variables selected for our model are the following:

- Expectedness, which represents the agent's level of expectation associated with the event.

- Likelihood, which represents the estimated probability of occurrence of the event.

- Desirability, which represents how desirable or undesirable the event is for the agent; it can be positive or negative.

- Causal attribution, which represents who is responsible for the event.

- Controllability, which represents the number of possible plans that the agent has to deal with for that event.

The use of fuzzy logic in the appraisal process facilitates the definition of fuzzy rules to generate emotions since fuzzy logic is closer to the way in which human beings express their emotions. Therefore, our fuzzy logicbased approach produces better and more reliable results than other nonfuzzy appraisal processes.

In our appraisal model, each event is associated with a tuple:

$$
\text { event } \leftarrow\langle e, l, d, a, c\rangle
$$

where $e, l, d, a$, and $c$ represent the fuzzy values for the appraisal variables Expectedness, Likelihood, Desirability, Causal_attribution, and Controllability, respectively. The possible values for these variables are:

$$
\begin{aligned}
& e, l, c \in\{\text { Low, Medium,High }\} \\
& d \in\{\text { High_desirable,Desirable, Low_desirable,Low_undesirable, } \\
& \text { Undesirable,High_undesirable } \\
& a \in\{\text { Self, Other }\}
\end{aligned}
$$




\subsection{A MODEL FOR EMOTION ELICITATION IN AFFECTIVE AGENTS}

On the other hand, an emotion is defined by a tuple:

$$
\text { emotion }=\langle\text { type, } \text { int }\rangle
$$

where type is the label representing the emotion type, and int represents the fuzzy intensity of the emotion:

$$
\begin{aligned}
& \text { type } \in\{\text { Happiness, Disgust, Sadness, Anger, Fear, Surprise, } \\
& \text { Calm, Boredom }\} \\
& \text { int } \in\{\text { Strong, Medium, Light, Neutral }\}
\end{aligned}
$$

The Neutral value is the default value for emotions that have not been elicited in the appraisal process. In addition, we have introduced a new appraisal variable named Time Without Events (TWE). This variable is not part of the event; it is an internal variable of the agent that measures the time that has passed since the last event. We have proposed this appraisal variable to elicit the calm and boredom emotions. The possible values for this variable, represented by $t$, are:

$$
t \in\{\text { Low, Medium, High }\}
$$

We use a fuzzy rule-based system to calculate the elicited emotion and its intensity. The general structure of a fuzzy rule [158] is:

$$
\begin{aligned}
& r_{i}: \text { IF } e \text { is } x_{e}^{i} \text { and } l \text { is } x_{l}^{i} \text { and } d \text { is } x_{d}^{i} \text { and } a \text { is } x_{a}^{i} \text { and } c \text { is } x_{c}^{i} \text { and } t \text { is } x_{t}^{i} \\
& \text { THEN type is } y_{\text {type }}^{i} \text { and int is } y_{\text {int }}^{i}
\end{aligned}
$$

where $r_{i}$ is the $i$ th fuzzy production rule, $x_{e}^{i}$ represents the fuzzy value for the Expectedness appraisal variable in the $i$ th rule, $x_{l}^{i}$ is the fuzzy value for Likelihood, $x_{d}^{i}$ is the fuzzy value for Desirability, $x_{a}^{i}$ is the fuzzy value for Causal attribution, $x_{c}^{i}$ is the fuzzy value for Controllability, $x_{t}^{i}$ is the $i$ th fuzzy value for Time Without Events, and $y_{\text {type }}^{i}$ and $y_{i n t}^{i}$ are the output values for the emotion type and the intensity calculated by the $i$ th rule (see Table 4.1). 
For example, a fuzzy rule appraising the Fear emotion with High intensity is defined as:

$r_{13}$ : IF $e$ is Low and $l$ is High and $d$ is High_Undesirable and $a$ is Other and $c$ is Low and $t$ is Low

THEN type is Fear and int is High

Table 4.1 summarizes the parameters of the 24 fuzzy rules that are proposed to define the appraisal process. Each row corresponds to a fuzzy rule, while each column contains the possible fuzzy values for each appraisal variable that is associated with each emotion. As we stated above, the definition of the appraisal variable values is based on the model proposed by Scherer [200] and the OCC model [163].

We have defined three fuzzy values for the intensity of each emotion. This intensity depends on the fuzzy values of the set of appraisal variables [148]. This fuzzy process not only derives emotions and their intensity from the set of appraisal variables, it also unifies the selection of the type and the intensity of the emotion in the same rule. Therefore, it is not necessary to define a new process to derive the intensity as in other models $[67,148]$.

\section{The Affect Adaptation process}

Two important factors that must be taken into consideration when modeling affective agents are mood and personality. Mood can be seen as the result of the various emotional events that are produced during a period of time. On the other hand, personality influences the type and intensity of the mood of a person [133]. For example, a person with high levels of neuroticism will be more likely to experience negative moods more frequently and with more intensity. In our model for affective agents, mood is represented in a twodimensional space in terms of Pleasure and Arousal. The Affect Adaptation process updates the mood by modifying the values of Pleasure and Arousal according to the current emotion, the agent's personality, and the language 


\subsection{A MODEL FOR EMOTION ELICITATION IN AFFECTIVE AGENTS}

Table 4.1: Fuzzy model for estimating the emotion and its intensity. Where $\mathrm{HD}=$ High desirable, $\mathrm{D}=$ Desirable, $\mathrm{LD}=$ Low Desirable, LU = Low Undesirable, $\mathrm{U}=$ Undesirable, and $\mathrm{HU}=$ High Undesirable.

\begin{tabular}{|c|c|c|c|c|c|c|c|}
\hline Emotion & Intensity & Expectedness & Likelihood & Desirability & $\begin{array}{l}\text { Causal } \\
\text { attribution }\end{array}$ & Controllability & TWE \\
\hline \multirow{3}{*}{ Happiness } & High & Medium & High & HD & & & \\
\hline & Medium & Medium/Low & High/Medium & $\mathrm{D}$ & & & \\
\hline & Low & Medium/Low & High/Medium & $\mathrm{LD}$ & & & \\
\hline \multirow{3}{*}{ Disgust } & High & Low & High & $\mathrm{HU}$ & Other & High/Medium & \\
\hline & Medium & Low & High & $\mathrm{U}$ & Other & High/Medium & \\
\hline & Low & Low & High & $\mathrm{LU}$ & Other & High/Medium & \\
\hline \multirow{3}{*}{ Sadness } & High & & High & $\mathrm{HU} / \mathrm{U}$ & & Low & \\
\hline & Medium & & High/Medium & $\mathrm{U}$ & & Low & \\
\hline & Low & & High/Medium/low & $\mathrm{LU}$ & & Low & \\
\hline \multirow{3}{*}{ Anger } & High & Medium & High & $\mathrm{HU}$ & & Medium & \\
\hline & Medium & Medium & High/Medium & $\mathrm{U}$ & & Medium & \\
\hline & Low & Medium & High/Medium/Low & $\mathrm{LU}$ & & Medium & \\
\hline \multirow{3}{*}{ Fear } & High & Low & High & $\mathrm{HU}$ & Other & Low & \\
\hline & Medium & Low & High/Medium & $\mathrm{U}$ & Other & Low & \\
\hline & Low & Low & High/Medium/Low & $\mathrm{LU}$ & Other & Low & \\
\hline \multirow{3}{*}{ Surprise } & High & Low & Low & & Other & & \\
\hline & Medium & Low & Medium & & Other & & \\
\hline & Low & Low & High & & Other & & \\
\hline \multirow{3}{*}{ Calm } & High & & & & & Medium & Low \\
\hline & Medium & & & & & Medium/Low & Medium \\
\hline & Low & & & & & Medium/Low & High \\
\hline \multirow{3}{*}{ Boredom } & High & & & & & Low & Low \\
\hline & Medium & & & & & Low & Medium \\
\hline & Low & & & & & Low & High \\
\hline
\end{tabular}

and culture in which the agent is located. We describe the different phases of this process in the following sections.

\section{Representing emotions in a Pleasure-Arousal space}

As we have shown in the previous sections, the result of the Event Appraisal process is the Appraised Emotion, which is composed of an emotion type and a fuzzy intensity. In the Affect Adaptation process, we use the Pleasure 


\section{CHAPTER 4. A FUZZY APPRAISAL MODEL FOR AFFECTIVE AGENTS (SELECTED PAPER)}

and Arousal dimensions of the Appraised Emotion to adapt the agent's mood. Therefore, to calculate how the mood will be modified by the Appraised Emotion, we need to represent this emotion in the same two-dimensional space that is used for representing the agent's mood. To avoid the loss of information, our multidimensional model must be able to represent both the emotion type and the emotion intensity.

Our proposal represents a fuzzy emotion in a two-dimensional space that is adapted to the culture and language in which the agent will be used. This model is based on the results of the experiment presented in [219]. In this experiment, one-hundred European Spanish-speakers, 40 females and 60 males ranging in age between 18 and 60 years old, were asked to assign fuzzy values of Pleasure and Arousal to ten terms/words expressing emotions in their mother tongue. The emotions selected for this experiment were: Happiness, Excitement, Surprise, Fear, Disgust, Anger, Sadness, Boredom, Calm, and Sleepiness. When the Pleasure and Arousal dimensions have low levels, the intensity of the emotion is so low that it can be considered that there is no emotion. Therefore, we discarded those responses whose values of Pleasure and Arousal were close to zero. We obtained the mean of the Pleasure and Arousal for each emotion, and then we calculated the mean and the standard deviation of the angle of each emotion in this two-dimensional space. This angle represents the meaning that each participant associates to each emotion. The results of the experiment are summarized in Table 4.2.

Our model also divides the Pleasure-Arousal space into four degrees of intensity: Strong, Medium, Light, and Neutral (Fig. 4.2). We have added the fuzzy value Neutral to refer to those emotions that are not intense enough to be considered as elicited. When we superimpose the results of the experiment on the model proposed for the intensity of emotions, we obtain different areas that relate the Pleasure and Arousal dimensions to each emotion label and its intensity adapted to the Spanish language. Fig. 4.3 shows the emotion model proposed in this paper, where the area assigned to each emotion, using one standard deviation, is represented in a different color. This model can be easily adapted to other languages and cultural environments using the results of similar experiments to assign different areas to each emotion. An agent with more than one Pleasure-Arousal space can easily adapt its emotion 


\subsection{A MODEL FOR EMOTION ELICITATION IN AFFECTIVE AGENTS}

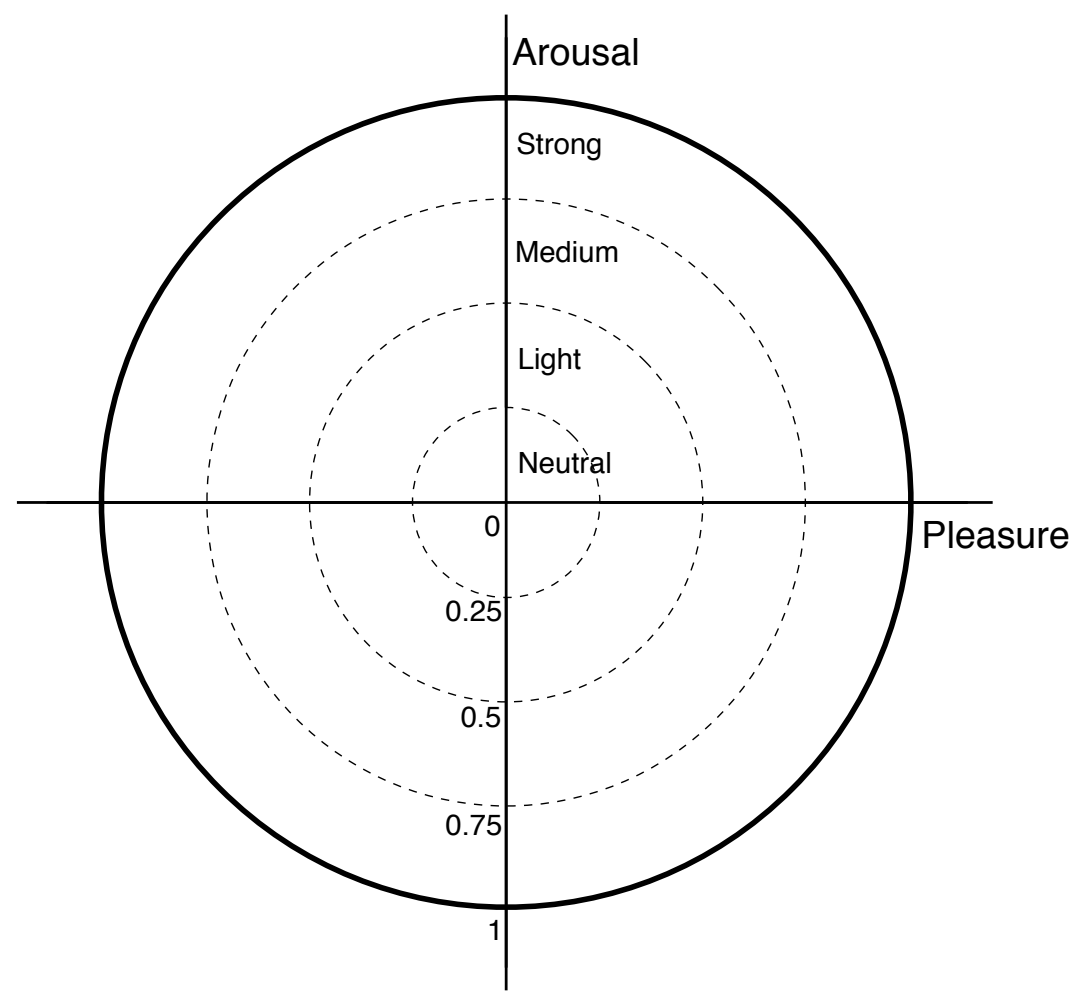

Figure 4.2: The proposed fuzzy model for the intensity of the emotions in the Pleasure-Arousal space.

expression depending on the language and culture of its interlocutor without modifying the rest of its affective components.

We have designed a defuzzification function that translates the fuzzy appraised emotion into its Pleasure and Arousal equivalent values. The defuzzification function determines the direction of the Appraised Emotion vector $\vec{e}$ in this culturally located, two-dimensional space using the mean angles obtained in the experiment for each emotion (see Table 4.2). For example, if the Appraised Emotion is Anger, the direction of the corresponding vector $\vec{e}$ will be 138.5 degrees, which corresponds to the mean angle obtained in the experiment for the Anger emotion . The defuzzification function also calculates the vector modulus, which indicates the intensity of the emotion, as the mean value for each intensity. For example, if the intensity of the Appraised Emotion is Strong, the modulus of the emotion vector $\vec{e}$ will be 0.875 , which 

AGENTS (SELECTED PAPER)

Table 4.2: Results of the experiment expressed in degrees.

\begin{tabular}{lrrrr}
\hline Emotion & Pleasure & Arousal & Mean angle & Standard deviation \\
\hline Happiness & 0.90 & 0.42 & 25.09 & 19.02 \\
Excitement & 0.76 & 0.64 & 39.97 & 10.32 \\
Surprise & 0.31 & 0.95 & 71.63 & 26.38 \\
Fear & -0.58 & 0.81 & 125.51 & 15.61 \\
Anger & -0.74 & 0.66 & 138.55 & 16.90 \\
Disgust & -0.99 & -0.04 & 182.58 & 43.65 \\
Sadness & -0.96 & -0.27 & 196.02 & 22.48 \\
Boredom & -0.41 & -0.91 & 245.34 & 21.41 \\
Sleepiness & -0.11 & -0.99 & 263.59 & 15.56 \\
Calm & 0.74 & -0.67 & 318.12 & 35.89 \\
\hline
\end{tabular}

corresponds to the midpoint of the Strong intensity that goes from 0.75 to 0.1. The result of this defuzzification function is the vector $\vec{e}$, which represents the Appraised Emotion. Considering that the horizontal axis in this two-dimensional space represents Pleasure and the vertical axis represents Arousal, if $\alpha$ is the angle of the vector $\vec{e}$, then the Pleasure and Arousal values of the Appraised Emotion can be calculated by a simple trigonometry formula as follows:

$$
(\text { Pleasure, Arousal })=(|\vec{e}| \cdot \cos \alpha,|\vec{e}| \cdot \sin \alpha)
$$

For instance, let us suppose an agent $a$ receives an event. The fuzzy appraisal process evaluates the values of the appraisal variables associated to that event and obtains the emotion $e$ defined by the type Anger and the intensity Strong by using the fuzzy rule-based system. The direction of the vector $\vec{e}$ corresponding to the Anger emotion is $\alpha=138.5$ (as shown in Table 4.2), and its modulus is $|\vec{e}|=0.875$. The agent can calculate the Pleasure and Arousal values associated with the emotion vector $\vec{e}$ using Formula 4.8: $($ Pleasure, Arousal $)=(-0.66,0.58)$.

We have shown how our model internally represents an emotion by a vector in which the modulus represents the intensity of the emotion and the direction 


\subsection{A MODEL FOR EMOTION ELICITATION IN AFFECTIVE AGENTS}

represents the type of emotion. The result is a circular representation of the emotions with some similarities with the scheme proposed by Russell (Fig. 4.1). However, as we stated above, Russell's scheme should not be used directly since it is only a scheme that was used to reinforce the idea that emotions follow a circular pattern; the area assigned to each emotion is more restricted, and the emotion intensity is not considered.

\section{Updating the mood}

Once the agent obtains the Pleasure and Arousal values of the appraised emotion, the agent can use these values to update its mood according to the agent's personality. In this phase, the vector of the agent's mood, which is also represented in the Pleasure-Arousal space, is "attracted" by the emotion vector to a greater or lesser extent depending on the agent's personality. We have defined two functions, called Mood_Resistance and Emotion_Influence, to model this behaviour. The Mood_Resistance function determines how a mood $m$ resists being changed by the appraised emotion. The Emotion_Influence function determines the influence or force of the emotion $e$ to modify the agent's mood. Both functions depend on the agent's personality. For example, an agent with a personality that is prone to negative affect will have a Mood_Resistance for the negative moods that is higher than the Mood_Resistance for the positive moods. That means that the agent will be more prone to suffer a negative mood with greater intensity than an agent with a personality that is prone to positive affect [216].

The current mood of an agent at instant $t, \vec{m}_{t}$, will be updated as a combination of the previous mood vector (mood at instant $t-1), \vec{m}_{t-1}$, weighted by the Mood_Resistance $(m)$ and the emotion vector $\vec{e}$ weighted by the Emotion_Influence(e) function:

$$
\vec{m}_{t}=\vec{m}_{t-1} \cdot \text { Mood_Resistance }\left(m_{t-1}\right)+\vec{e} \cdot \operatorname{Emotion\_ Influence}(e)
$$

where Mood_Resistance $\left(m_{t-1}\right)$ and Emotion_Influence $(e)$ represent how the personality of the agent affects its mood. These two functions are calcu- 


\section{CHAPTER 4. A FUZZY APPRAISAL MODEL FOR AFFECTIVE AGENTS (SELECTED PAPER)}

lated using the following formulas:

$$
\operatorname{Mood\_ Resistance}(m)=\frac{\sum_{p \in P} \beta_{p} \cdot \theta_{m, p}}{\sum_{p \in P} \theta_{m, p}}
$$

$$
\text { Emotion_Influence }(e)=\frac{\sum_{p \in P} \beta_{p} \cdot \theta_{e, p}}{\sum_{p \in P} \theta_{e, p}}
$$

where $P$ represents the set of personality traits of an agent. For example, for the five-factor model (FFM), these traits are Openness $(\mathrm{O})$, Conscientiousness (C), Extraversion (E), Agreeableness (A), and Neuroticism (N); therefore, $P$ is defined by the set $P=\langle O, C, E, A, N\rangle$. $\beta_{p}$ is the value of a personality trait $p \in P . \theta_{e, p}$ is a weight that relates the personality trait $p$ with the emotion $e$. There will be one weight $\theta_{e, p}$ for each pair of emotion type and personality trait $(e, p)$, but many of these weights could be zero. Each weight $\theta_{e, p}$ determines how the personality trait $p$ influences the emotion type $e$. Therefore, the set of weights $\theta_{e, p}$ can be viewed as the set of correlations between emotions and personality traits: the greater the correlation between the personality trait $p$ and the emotion type $e$, the greater the value of $\theta_{e, p}$. For example, considering that the trait of Extraversion $E$ is related to positive emotions and Neuroticism $N$ does not have a very high relation with emotions of this type, for the Happiness emotion, the value of the weight $\theta_{\text {Happiness,E }}$ will be greater than the value of the weight $\theta_{\text {Happiness }, N}$. Different $\theta_{e, p}$ values can be chosen by the agent programmer to generate agents with different emotional behaviors and personalities.

To better understand this method, let us reconsider the previous example where after evaluating an event at instant $t-1$, an agent $a 1$, has elicited the Appraised Emotion represented by vector $\vec{e}$ in Fig. 4.3. Let us consider that the mood for agent $a 1$ at instant $t-1$ is defined by the vector $\vec{m}_{t-1, a 1}$ - The Pleasure and Arousal components for the mood vector $\vec{m}_{t-1, a 1}$ are $(-0.30,-0.20)$. Let us also consider a second agent, $a 2$, who has appraised the same emotion as agent $a 1(\vec{e})$ and has the same mood. To simplify, we will represent the mood of both agents by $\vec{m}_{t-1}\left(\vec{m}_{t-1}=\vec{m}_{t-1, a 1}=\vec{m}_{t-1, a 2}\right)$. The 


\subsection{A MODEL FOR EMOTION ELICITATION IN AFFECTIVE AGENTS}

Table 4.3: Example of $\theta_{e, p}$ values.

\begin{tabular}{lll}
\hline Emotion & $\mathrm{E}$ & $\mathrm{N}$ \\
\hline Anger & $\theta_{\text {Anger }, E}=0.5$ & $\theta_{\text {Anger }, N}=0.8$ \\
Sadness & $\theta_{\text {Sadness }, E}=0.6$ & $\theta_{\text {Sadness }, N}=0.7$ \\
\hline
\end{tabular}

Affect Adaptation process will update the agents mood at instant $t$ taking into account the effect of the Appraised Emotion, the mood of the agents at instant $t-1$, and the personality of the agents. To simplify this example, the agent's personality is defined using only two traits: Extraversion (E), which is related to positive affect; and Neuroticism $(\mathrm{N})$, which is related to negative affect. Therefore, the personality in this example is defined by the tuple:

$$
P=\langle E, N\rangle
$$

On the other hand, for this example, we consider the values for $\theta_{e, p}$ shown in Table 4.3. These values are based on the results obtained from the experiments presented in [75], making them consistent with theories that associate Extraversion with positive emotions and Neuroticism with negative emotions [206]. Taking into account these values, if we define the personality of agent $a 1$ by a level of Extraversion of 0.9 and a level of Neuroticism of 0.1 $\left(P_{a 1}=(0.9,0.1)\right)$ and the personality of agent $a 2$ by a level of Extraversion of 0.1 and a level of Neuroticism of $0.9\left(P_{a 2}=(0.1,0.9)\right)$, then the values of Pleasure and Arousal for the new mood of agents $a 1$ and $a 2$ at instant $t$ calculated by Formula 4.9 are $\vec{m}_{t, a 1}=(-0.4,0.1)$ and $\vec{m}_{t, a 2}=(-0.6,0.3)$. These values are represented in Fig. 4.3 by the vectors $\vec{m}_{t, a 1}$ and $\vec{m}_{t, a 2}$.

Comparing the resulting moods of both agents, it can be observed that, in the case of agent $a 1$, which has a high level of extraversion, the mood has been less affected by a negative emotion than in the case of agent $a 2$, which has a higher level of neuroticism. These results are consistent with theories that claim that personalities with low Extraversion and high Neuroticism are more prone to negative moods $[27,56]$.

As we have shown in our proposal, the mood is adapted to the current emotion according to the agent's personality. Therefore, our model facilitates the development of emotional multi-agent systems since it allows different moods 
to be easily obtained for each agent based on the agent's personality. Different moods will produce different agent behaviors, which will improve the agent's capability to simulate human behaviors [216]. For example, an agent with a negative personality will be more prone to negative moods than an agent with a positive personality. Therefore, from psychological and neurological perspectives, this proposal is more consistent with the different theories that argue that emotions and personality have an effect on mood [133] and it also explains why people are more or less likely to have certain types of mood.

\section{The Fuzzification Process}

In our model, the pair (Pleasure, Arousal) is internally used by an agent to represent the appraised emotion and its mood in the two-dimensional space. When the agent needs to express its mood, it has to perform a fuzzification process to determine the emotion type and intensity corresponding to the mood vector defined by the pair (Pleasure, Arousal). The intensity of the emotion is easily calculated using the modulus of the emotion vector and the fuzzy model represented in Fig. 4.2. On the other hand, the process to calculate the emotion type corresponding to an emotion vector can be viewed as a classification problem where there is a set $C$ of ten classes that correspond to the ten emotions.

$$
\begin{aligned}
C \in\{ & \{\text { Happiness, Excitement, Surprise, Fear, Anger, Disgust }, \\
& \text { Sadness, Boredom, Sleepiness, Calm }\}
\end{aligned}
$$

Using the Gaussian models obtained from the experiment for Spanishspeakers (Table 4.2), we can estimate the probability of each emotion. We have defined the $t(\vec{v})$ function as a Bayesian classifier that returns the emotion type with the maximum likelihood for an angle $\alpha$ of the emotion vector $\vec{v}$ :

$$
t(\vec{v})=\underset{c}{\arg \max } \widehat{P}(C=c \mid \alpha)
$$

where $c \in C$, and $\widehat{P}(C=c \mid \alpha)$ represents the estimated conditional probability of the class of emotion $c$ for the angle $\alpha$. This probability is 


\subsection{A MODEL FOR EMOTION ELICITATION IN AFFECTIVE} AGENTS

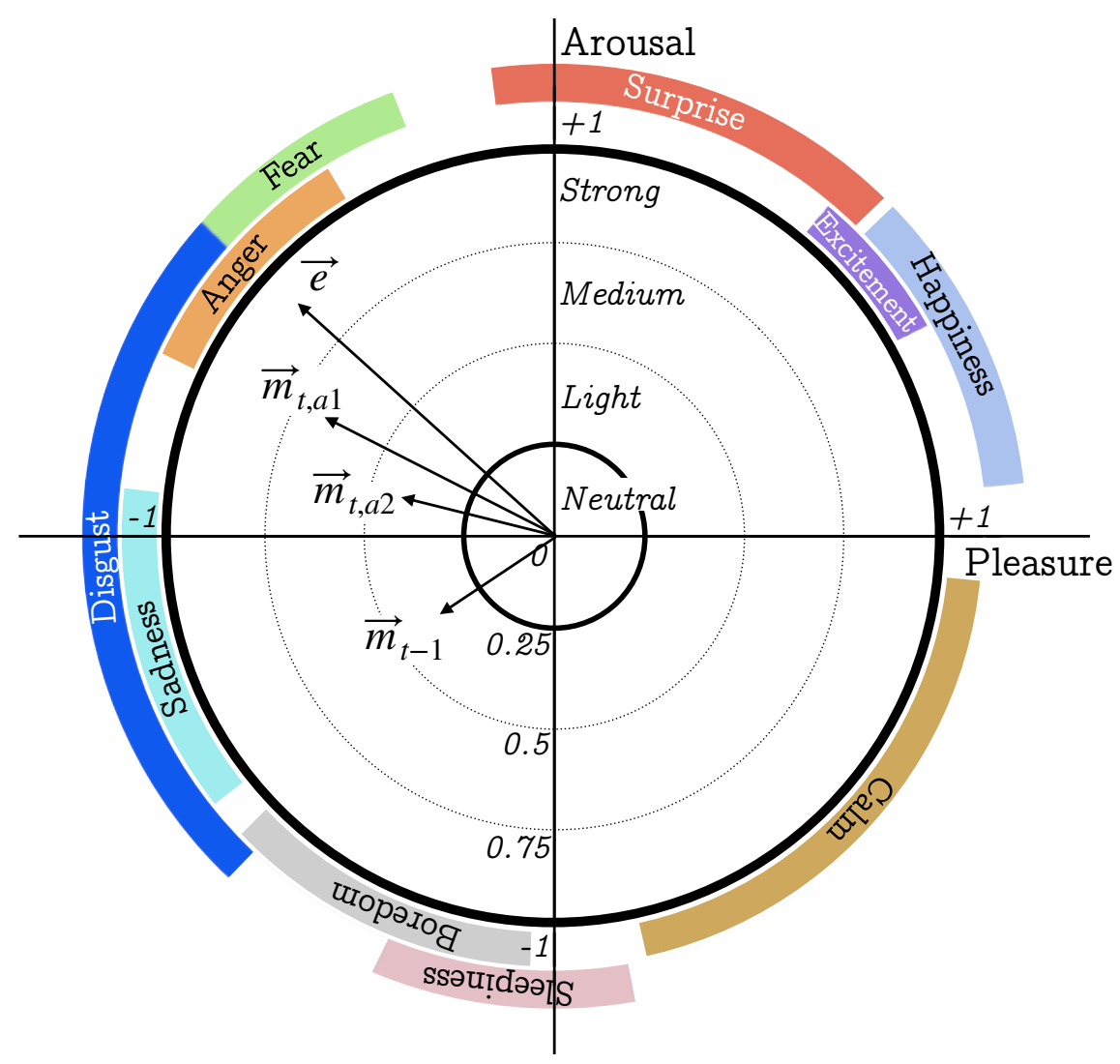

Figure 4.3: Example of the estimation of the mood considering two different personalities.

estimated by the normal distributions obtained in the experiment as:

$$
\widehat{P}(C=c \mid \alpha) \sim f\left(\alpha \mid \mu_{c}, \sigma_{c}\right)
$$

where $\mu_{c}$ and $\sigma_{c}$ represent the mean and the standard deviation of the class of emotion $c$. $\widehat{P}(C=c \mid \alpha)$ is estimated using the probability density function of a Gaussian distribution $f\left(\alpha \mid \mu_{c}, \sigma_{c}\right)[112]$ :

$$
f\left(\alpha \mid \mu_{c}, \sigma_{c}\right)=\frac{1}{\sigma_{c} \cdot \sqrt{2 \pi}} \cdot e^{-\frac{\left(\alpha-\mu_{c}\right)^{2}}{2 \cdot \sigma_{c}^{2}}}
$$

Continuing with the previous example, if agent $a$ wants to express the emotion $\vec{e}=(-0.66,0,58)$ (see Figure 4.3$)$, the agent will calculate the most probable labels for the type and the intensity of the emotion through the 
Fuzzification Process. First, the modulus of the vector is calculated (in this example, $|\vec{e}|=0.875)$ to obtain the fuzzy intensity. In this case, since the module is in the range of the Strong intensity $(0.75 \leq|\vec{e}| \leq 1)$, the label of the emotion intensity is Strong. Then, the label of the emotion type is obtained using Formula 4.14 and the Gaussian models obtained from the experiment in the same cultural environment in which the agent is located. In this case, the maximum argument for the function $t(\vec{e})$ is $\widehat{P}(C=$ Anger $\alpha=138.5)$. Therefore, the most likely label for that emotion is Anger.

Traditionally, in affective computing, most of the proposed appraisal models calculate the intensity and the emotion type of emotions independently by using numerical variables. Our model unifies the appraisal of the type and intensity of the emotion in the same rule. Dimensional models, such as the one proposed in this paper, also allow the affective behavior of the agent to be described in a more precise way. This dimensional representation defines regions where emotions can occur and therefore represents the proximity between two emotions and the intensity of the emotions. This representation allows the simulation of processes such as the decay rate of the mood over time and opens the doors to the simulation of new processes such as empathy or emotional contagion.

\subsection{Conclusions and future work}

In this paper, we have proposed a new fuzzy appraisal model using the Pleasure and Arousal dimensions, which is adapted by a defuzzification process to the culture and language in which the agent is located. Our model uses fuzzy logic to better reproduce the way in which humans express emotions. We propose two processes to simulate affective behavior. When an event is perceived, the Event Appraisal process evaluates five fuzzy appraisal variables to obtain the fuzzy Appraised Emotion. Then, the Affect Adaptation process defuzzificates the Appraised Emotion and adapts the mood using this defuzzificated Appraised Emotion, the agent's personality, and the cultural environment. This Affect Adaptation process allows different agents to have different moods when they perceive the same event, which will allow agents 


\subsection{CONCLUSIONS AND FUTURE WORK}

to show different behaviors depending on the personality of each agent.

The proposed appraisal model has two important advantages. It can be easily adapted to trigger more than one emotion in each appraisal cycle, and it is capable of eliciting ten different types of emotions with different intensities despite having an objectively small number of fuzzy rules. Our model uses the Pleasure and Arousal dimensions internally to define emotions and mood instead of simple labels. With these dimensions, emotions and mood can be defined as vectors in which the modulus represents the intensity of the emotion, while the type of emotion is represented by the direction of this vector. Through this dimensional representation, the variables representing affective characteristics of agents (emotion and mood) can be easily adapted to obtain different emotions and moods. Therefore, our model improves the representation of emotions because it allows emotions and mood to be modified, adapted, and stored using a dimensional representation. In this work, we have also defined a fuzzy model of intensity that depends on the modulus of the emotion's vector. This model allows the agent to express emotions in the same way as humans.

One important novelty of our proposal is that our model allows the agent to express its emotional state using the same interpretation of the fuzzy emotional terms used in the language and culture of its human interlocutor. This has been achieved by defining the two-dimensional Pleasure-Arousal space using the results of an experiment with participants of the same cultural environment in which the agent is located. Moreover, one agent can easily adapt the expression of its emotions to other cultural environments using different Pleasure-Arousal spaces that can be easily incorporated to its affective component.

We are currently integrating our emotional model in the affective agent architecture $G e n I A^{3}$ in order to allow the development of different behaviors depending on the agent's personality and mood. We are interested in defining a new Pleasure-Arousal space that is adapted to other language and/or cultural environments. This will allow agents to adapt their emotion expression to interlocutors of different cultural environments. We are also interested in analyzing the effect of adding the Dominance dimension to the Pleasure-Arousal space. We think that this additional dimension could 
disambiguate the overlaps that occur between some emotions in the twodimensional model. 



\title{
Chapter 5
}

\section{A formal extension of the agent-oriented programming language agentSpeak for modelling affective agents with empathic abilities}

\begin{abstract}
In this paper, we present a formalization of an extension of the syntax, the semantics, and the reasoning cycle of AgentSpeak. Our proposal modifies the AgentSpeak inference model to provide support to the development of empathic agents. The new inference model allows empathy to affect the agent's deliberation and reasoning processes to select plans according to the analyzed event and the affective state and personality of the agent. In addition, our proposal allows a software agent to simulate the distinction between self and other agents through two different event appraisal processes: the empathic appraisal process, for eliciting emotions as a response to other agents emotions, and the regular affective appraisal process for other non-empathic affective events. The empathic regulation process adapts the elicited empathic emotion based on different factors including intrapersonal (e.g., the agent's personality and affective memory) and interpersonal (e.g., the affective link between the agents) characteristics of the agent. The use of a memory of past events and the corresponding elicited emotions allows the maintaining of an affective link to support long-term interaction between agents.
\end{abstract}




\subsection{INTRODUCTION}

\subsection{Introduction}

Computer systems that are oriented to the paradigm of human-machine interaction are becoming more relevant in society. Systems of this type are progressively becoming more complex and require higher level abstractions and metaphors to describe capabilities and characteristics that cannot be explained by classical lower level specifications. It is now possible to reflect the way in which human beings understand the world more faithfully through these abstractions and metaphors. Endowing software agents with affective abilities, particularly empathic abilities, is one of the metaphors that will make it possible to transform the human-machine interaction paradigm by making it more human-oriented. Empathy is a concept that has evolved over the years, whose meaning (which is still being discussed) refers to a wide range of affective and cognitive competencies that are fundamental in the development of the human being as a social being [98]. These competencies allow one "to put oneself in others' shoes" and to understand and share their feelings and thoughts, which generates a series of pro-social behaviours aimed at improving their well-being [50]. Empathy provides a medium for the establishment of affective links that support moral and social behaviour [22]. Therefore, empathy is a key element in social interactions affecting human affective states and behaviours, providing a basis for long-term relationships. In fact, social disorders, such as autism spectrum disorder, are related to a deficit in empathic abilities [95]. In any empathic interaction, we can distinguish two actors: the target actor, who is the person or agent that suffers the effect of an action or situation, and the observer, who is the person that perceives that action or situation and feels empathy for the target person.

Different theoretical approaches that try to explain the cognitive processes related to empathy have been proposed in psychology, sociology, philosophy, ethology, and neuroscience. The most recent theoretical approaches provide a conceptualization of empathic behavior from a perspective derived from appraisal theories that relate the emergence of emotions to a cognitive appraisal of an event $[135,72]$. Under this perspective, empathy arises from the appraisal of the perception of a situation or an emotion in others [231, 96]. Moreover, according to several authors, empathy is affected by a regulation 


\section{CHAPTER 5. A FORMAL EXTENSION FOR MODELLING EMPATHIC AGENTS}

process that modulates the empathic response to an event according to different factors, including intrapersonal (e.g., personality) and interpersonal (e.g., affective link with the target) factors [50, 54, 43].

For years, the inherent characteristics of human affective behavior have been the subject of research in the field of affective computing [170] with the aim of improving simulations of human behaviour and human-machine interactions $[7,148]$. In recent years, there has been a growing trend towards the development of models that are capable of simulating empathic abilities $[165,236]$. Most of these models use the agent-oriented paradigm. Empathic agents have been shown in multiple experiments to improve the user experience in human-machine interactions $[188,161,159]$. Other experiments, such as the one presented in [138], have also demonstrated the importance of modelling long-term interactions to improve the simulation of empathic behavior in empathic agents. In general, these models are designed to be implemented in an ad hoc manner making the agent specification programmer-dependent $[31,30]$. However, an empathic agent model that is based on well-known agent-oriented languages such as AgentSpeak [179] could provide a basis for the development of agents with rational and affective abilities. This could reduce the dependency on the programmer and thus increase usability.

In this paper, we propose an empathic agent model that is designed for AgentSpeak. Our model is based on recent empathic appraisal theories to elicit empathic emotions when a software agent perceives or is aware of an emotion or a situation in other agents or humans. In addition, our empathic agent model has an emotion regulation process that adapts emotions to different intrapersonal and interpersonal factors. The rest of this paper is organized as follows. In Section 5.2, we perform an introduction to the theoretical frameworks supporting affective states and empathy, and we discuss the proposals made in affective computing to simulate empathic behavior. Section 5.3 introduces our AgentSpeak extension to enable the development of agents with empathic abilities. Finally, the main conclusions and some future works are presented in Section 5.4. 


\subsection{Related work}

Human beings are emotional beings, and different affective characteristics motivate human behavior. Traditionally, the simulation of human behavior through computational systems has generally focused on practical reasoning. However, classical practical reasoning has some limitations when simulating non-rational behaviors such as affective behaviors. For years, the area of affective computing [170] has been working to design models to understand and simulate these affective behaviors [7, 148, 186, 82, 220]. These models generally include different constructs such as affective states, personality, or empathy that come from different branches of psychology, ethology, philosophy, and sociology. Affective states, which encompass emotions and moods, are complex constructs whose definition remains a subject of debate to this day. Over the years, different scientists have tried to provide an answer to the phenomenon of emotions and moods. Although emotions are generally considered to be quick responses to certain stimuli, there are different approaches that try to explain how this phenomenon arises. One of the most recognized theories is the appraisal theory $[135,72]$, according to which, when a stimulus is received, an appraisal process occurs, resulting in an emotion. These theories are based on the existence of different variables known as Appraisal Variables. Different authors differ in the number and type of Appraisal Variables involved in the emotion generation process. For example, K.R. Scherer [200] proposed a model in which twenty-two Appraisal Variables were defined, while A. Ortony, G. Clore, and A. Collins [163] proposed a more simplified model, known as OCC, with only eight Appraisal Variables. However, appraisal theories are not the only theories that have been developed to explain the phenomenon of emotion. Basic emotion theories relate events to specific emotions using a limited number of emotions. For example, the basic theory of emotions proposed by P. Ekman [65] uses six basic emotions: Happiness, Surprise, Fear, Anger, Disgust, and Sadness. In contrast to these basic emotion theories, the constructivist theories advocate for a more universal concept of emotions. Constructivism holds the existence of an unlimited number of emotions due to cross-cultural differences [196]. One of the most recognized constructivists is J.A. Russell who proposed the Circumplex Model of Affect 


\section{CHAPTER 5. A FORMAL EXTENSION FOR MODELLING EMPATHIC AGENTS}

[193]. According to Russell, emotions can be expressed in a two-dimensional space that is composed of the dimensions of Pleasure and Arousal. This theory is in line with the findings of neuroscience made in recent years [174]. Other authors have proposed different dimensions to explain emotions. For example, A. Mehrabian [154] added a third dimension, called Dominance, that helps to disambiguate some emotions such as Fear and Anger.

On the other hand, although the differences between emotions and mood continue to divide the academic community, it is generally accepted that, in contrast to emotions, mood is not related to a specific stimulus but rather arises as a consequence of the succession of different stimuli. Mood also has a longer duration (from minutes to days) and a lower intensity than emotions [19]. The variation in mood duration is sometimes attributed to the time the mood spends in transitioning to a neutral state or equilibrium state [167]. Both the equilibrium state and the speed of transition may vary depending on the individual's affective characteristics. Moreover, it should be considered that both emotions and mood are influenced by personality [197]. Different studies over the years have shown that personality influences emotional behavior $[64,134]$. Personality can make an individual more or less prone to certain emotions or moods, including complete suppression of certain emotions or moods [133]. Thus, personality acts as a factor in the regulation of affective behaviors offering an explanation for the observed differences in individual attitudes when facing the same situation [91]. One of the most recurrent theories of personality is the Five Factor Model (FFM), which is also known as the OCEAN model [152]. This model defines personality through five dimensions: Openness, Conscientiousness, Extraversion, Agreeableness, and Neuroticism. These five dimensions have an effect on the affective states that can be elicited in an individual. Apparently, the most evident relationships arise when relating positive affective states to Extraversion and when relating negative affective states to Neuroticism [56].

Finally, empathy is a construct that is used in different domains, such as psychology, ethology, sociology, or philosophy, to describe a variety of psychological attitudes that enable the development of social individuals. In general terms, empathy is an ability that allows humans to understand and feel the affective state of others, resulting in behavior directed toward mu- 


\subsection{RELATED WORK}

tual understanding. Several authors consider empathy to be a fundamental psychological ability that allows the establishment and maintenance of social bonds $[22,100,184,157,205]$. Empathy also plays a fundamental role in our society, affecting both morality and mutual understanding. [99]. In fact, empathy appears to be the basis on which social behaviors and interpersonal interactions are founded [50, 149]. Empathy connects people by promoting relationships and collaborations through the exchange of experiences, needs, and desires. [184]. However, the definition of the term empathy continues to divide the academic community [164]. This is largely due to the fact that the concept of empathy encompasses a large number of emotional, ethical, moral, and social factors. This lack of consensus can be clearly seen in the review conducted in [49], in which the authors identified forty-three different definitions for the concept of empathy.

The modern concept of empathy is attributed to psychologist E. Titchener who translates the concept "Einfühlung" proposed by the German philosopher R. Vischer and later expanded by T. Lipps through his theory of "Einfühlung" $[78,229]$. In this earlier definition, empathy was described as an innate instinct that produces a self-awareness in the experience and awareness of the target in the observer without any perspective-taking, associative, or cognitive process $[230,213]$. This concept evolved over the years in different theories until the discovery of mirror neurons [187] at the end of the 20th century, which reopened the debate on the conceptualization of empathy. Mirror neurons establish a relationship between perception and action that has led to a greater understanding of how concepts such as imitation and empathy are produced in the brain and are related to emotion experiences [104, 73, 130, 62, 70]. Based on this relationship between perception and action, Preston and de Waal [176] proposed a theory of empathy known as the Perception Action Model (PAM). According to the PAM, when the observer perceives an emotion in the target, he/she can experience the same emotion automatically and not consciously, producing a matching of mental states between the observer and the target [175].

Nowadays, theories that use a multidimensional perspective in which empathy is embedded in a functional sense and related to high level cognitive abilities are becoming more and more relevant $[226,145]$. Some of these the- 


\section{CHAPTER 5. A FORMAL EXTENSION FOR MODELLING EMPATHIC AGENTS}

ories argue that, if empathy was an automatic process resulting from direct perception as suggested by the PAM, then humans would be constantly empathizing $[96,52]$. However, there are situations in which empathy is inhibited for several reasons. This inhibition is produced by an adaptation resulting from a cognitive process known as empathic regulation. For instance, professionals in psychiatry are able to distance themselves from the patient's emotions [94]. F. De Vignemont and T. Singer [52] provided an explanation for this phenomenon by identifying two types of empathic regulation: empathy can be regulated by a voluntary process of self-regulation that improves with experience, as is the case of professionals in psychiatry; or there is an automatic and involuntary process that the authors call implicit appraisal, which depends on different regulation factors. Factors that influence empathy regulation have been widely discussed in academia $[231,176,53,51,55]$. In general, these regulation factors can be grouped into three categories: factors related to the internal characteristics of the observer such as personality, mood, age, life experiences, or gender; situative context factors such as the existence of more than one target suffering different experiences, which makes it difficult to empathize; and factors concerning the level of relationship between the observer and the target. This last category encompasses different interpersonal factors such as: similarity, which are the perceived similarities between the observer and the target (e.g., gender, personality, mood, or age); familiarity, which is related to the observer's previous experiences with the target in similar situations; and the affective link, which defines the affective relationship established between the observer and the target derived from the interactions they have over time [54].

Empathy needs to be dissociated from other social emotions. For instance, some authors have shown that empathy and Theory of Mind (or perspectivetaking) correspond to different processes, recruiting different brain regions $[145,212,125]$. The ToM holds that people are capable of understanding the mental states of others thanks to a system of rules based on their own experiences $[226,94,76]$. This process allows an observer to imagine what the target is thinking or feeling. Furthermore, most theorists generally agree that, to be able to distinguish a non-empathic emotion from an empathic emotion, the observer needs to be able to maintain a sense of self as distinct 


\subsection{RELATED WORK}

from the target [231].

New advances focus on the reasoning behind empathy inhibition as evidence that empathy is much more than responses to a stimulus [50, 231, 96, 43]. Under this perspective, empathy is described as an appraisal process that is based on other-oriented perspective-taking, self-oriented perspectivetaking, and ToM to try to understand the affective situation of the target. The perspective-taking does not always require the observer to perceive the target's affective states. It is described as a process of higher level understanding of the target's situation allowing other affective experiences such as vicarious emotions $[164,10,129,45]$ (e.g., when we are happy because someone has achieved a goal). Therefore, the process of appraisal and regulation of empathy includes non-affective information such as beliefs, desires, and goals [86]. Following this premise, J.D. Wondra [231] proposes an empathic appraisal model to explain the differences observed when the emotion elicited in the observer does not correspond to the emotion observed in the target. Similarly, C. Heyes [96] presents an appraisal-based system with a process that controls the empathic response.

\section{Empathy in software agents}

The relationship of empathy with social behavior and interpersonal relationships has aroused the interest of many researchers who focuses on the areas of human behavior simulation and human-machine interaction [161, 138]. Systems that are capable of simulating empathic abilities have been used in different contexts and have proved to be more reliable and more credible, thus reducing the stress and frustration of human users and improving humanmachine interaction [165]. For example, in the study conducted in [159], a robot with empathic abilities was used in an educational environment. The study determined that the empathic robot was able to elicit and maintain the social engagement of the participants of the experiment. Similarly, the experiments conducted with empathic agents in [161] showed that participants had a better perception of the agent when it displayed empathic emotions. Also, the research performed in [188] showed that agents with empathic abilities increased the involvement and sociability of participants. Therefore, consid- 


\section{CHAPTER 5. A FORMAL EXTENSION FOR MODELLING EMPATHIC AGENTS}

ering these results, it is not surprising that in the search for improvement in the simulation of human behavior and human-machine interaction, different proposals for computer systems with empathic abilities have appeared over the years. For example, Ö.N. Yalçın and S. DiPaola [235] introduced a model of an empathic agent based on the Russian Doll model [53]. The Russian Doll model is a psychological theory derived from the PAM model [176] that explains high level cognitive processes. This model uses the analogy of the Russian Doll, stratifying the processes related to empathy in different layers. The inner layers represent the most primitive mechanisms of empathy related to the PAM such as mimicry or emotional contagion. The top layers represent higher level processes such as perspective-taking. Following this reasoning, Yalçın and DiPaola proposed a model that is composed of three layers. In the bottom layer, the communication competence contains the processes related to the recognition and the expression of emotions. The middle layer represents emotion regulation processes. Finally, the top layer, called cognitive mechanisms, includes ToM and appraisal.

Emotion recognition has traditionally played a central role in the design of empathic agents. For example the work in [181] presents a model for an empathic agent that was based on the perception of the target's emotion to simulate an empathic interaction. The system recognizes the target's emotion and makes an estimation of the empathic emotion by means of a regulation process that uses the agent's personality and mood as regulation factors. They use the mapping proposed in [153] to establish the relationship of personality and mood in a two-dimensional space that is based on Pleasure and Arousal. Another interesting approach can be seen in [31] in which an embodied virtual agent model with empathic abilities known as EMMA (Empathic MultiModal Agent) is presented. In that model, an agent is essentially composed of a reasoning module and an empathic module. The reasoning module is based on the BDI (beliefs, desires, and intentions) architecture [180]. The empathic module has a facial expression recognition system and an empathetic appraisal process that is based on the De Vignemont and Singer theory [52]. To estimate the empathic emotion, the agent attempts to internally mimic the recognized facial expression using a set of patterns called Action Units (UAs). Subsequently, the emotion is regulated in an emotion regulation 


\subsection{RELATED WORK}

process that is based on the PAD model [154]. This process modifies the PAD components of emotions by means of a set of regulation factors that include similarity, mood, and deservedness (which represents the degree to which the target deserves or does not deserve the event). Finally, the elicited empathic emotion is translated into an Action Unit and shown in the agent's facial expression. Other models focus more on the simulation of empathy through the simulation of high-level cognitive processes. For instance, the model presented in [188], which also relies on the theory of De Vignemont and Singer to define the empathic process, focuses on the self-projection appraisal based on OCC to elicit the empathic emotion. The appraisal process is based on a set of predefined rules that define the relationship of events to desirability. The elicited emotion is adapted by using the affective link, similarity, mood, and personality in an empathic regulation process. Similarity is obtained by comparing the intensity and valence of both the perceived and the elicited emotions. Personality is composed of a set of thresholds for each emotion. However, these factors only affect the intensity of the emotion elicited in the empathic appraisal. Another example of the simulation of cognitive processes can be found in [161] in which a model of an embodied empathic dialogue agent is presented. The purpose of this agent was to simulate an empathic interaction with human users. The agent deduced the user's emotions through the dialogue using a perspective-taking strategy and then responded by showing the same emotion. The agent was able to regulate the intensity of the empathic emotion based on a preset degree of empathy between the agent and the user. Experiments conducted with the agent showed that participants had a better perception of the agent when it displayed empathic emotions.

Different authors have emphasized the need to consider the inclusion of long-term interactions to improve the simulation of empathic interactions $[165,139,117]$. However, not much work has been done on this topic. For instance, in [138] a model of empathy based on emotion recognition is proposed. The system is based on a robot called iCat that interacts with children while playing chess. The iCat maintains the actions that children perform in chess moves in its memory and uses this information to determine the next move taking into account the children's emotions.

From what has been explained above, it can be deduced that most of the 


\section{CHAPTER 5. A FORMAL EXTENSION FOR MODELLING EMPATHIC AGENTS}

models of agents with empathic abilities use three fundamental processes: a perception process, in which an emotion or situation is perceived; an empathic appraisal process, in which the empathic emotion is elicited; and an emotion regulation process, in which the emotion is adapted to different regulation factors.

On the other hand, there is a wide range of proposals that focus on the agent-oriented programming (OAP) paradigm to develop agents with empathic abilities. Most of these proposed models are built ad hoc to simulate a certain empathic behavior, making the agent specification highly dependent on the programmer. However, the use of a commonly-used agent-oriented programming language can help to improve the development of empathic agents. In addition, these languages generally include support for processes such as perception, multi-agent communication, or rational behavior. One of the most well-known agent-oriented programming languages is AgentSpeak [179]. AgentSpeak is a language that is based on the logic programming paradigm for developing agents based on a BDI architecture. The BDI architecture provides the basis for the development of agents based on practical reasoning. Practical reasoning is an inference process through which agents evaluate and weigh their options taking into consideration the context of the practical situation in which they are involved and their knowledge about the environment. The result of this inference process is the modification, deletion, or addition of beliefs and intentions, altering the mental state of the agent. A deeper interpretation of the concept of practical reasoning allows the definition of two basic actions: deliberation and means-end reasoning. The deliberation process allows the agent to choose the state that it wants to achieve, while the means-end reasoning focuses on the actions that are required to achieve that state. The are some approaches that have used the BDI model to design agents with affective capacities $[31,6]$. For example, in [85] an appraisal process based on the OCC model for eliciting emotions in BDI agents is proposed. However, that work use only one appraisal process without considering the empathic emotions.

Over the years, the AgentSpeak language has been extended by different researchers to improve its usability $[224,26,24]$. The majority of these extensions were focused on the development of Jason [25]. Jason is a platform for 


\subsection{FORMALIZATION OF THE AGENTSPEAK EXTENSION TO DEVELOP EMPATHIC AGENTS}

the development of multi-agent systems implemented in Java whose core is an AgentSpeak interpreter. This platform enables the development of rational agents based on practical reasoning.

Despite the advantages provided by a well-known language such as AgentSpeak, there are very few proposals that use it when defining empathic agents. In [113], an empathic agent model based on AgentSpeak is presented for the resolution of conflicts of interest in interactions between agents. However, this approach focuses on utility-based functions to find solutions that are mutually acceptable, but no affective characteristic of the agents is considered in the utility functions. One of the most significant efforts to allow the use of affective agents in AgentSpeak is the GenIA ${ }^{3}$ architecture [6]. GenIA ${ }^{3}$ is a general-purpose architecture that extends Jason providing a platform that is easily adaptable to different theories of emotion in order to facilitate the development of affective agents. GenI ${ }^{3}$ adds new processes for the management of affective states, which are described in detail in Section 5.3. All of the new affective processes are grouped together to constitute the affective cycle of an agent. This affective cycle runs parallel to the rational cycle, allowing the agent to evaluate events at an affective level and consequently modify its behavior to cope with these events. However, before the work presented here, this architecture did not provide the necessary support to the development of agents with empathic abilities.

\subsection{Formalization of the AgentSpeak extension to develop empathic agents}

As discussed above, the use of high-level abstractions and metaphors, such as empathy, in the definition of human-oriented systems contributes to improving the experience of human-machine interactions and to make software agents more realistic when modelling human organisations. One of the most widely used paradigms proposed to model rational and affective behaviour is the agent-oriented paradigm. As stated in the previous sections, some proposals have incorporated affective characteristics into BDI-based agents; however, only few of them consider the modelization of empathic and ratio- 


\section{CHAPTER 5. A FORMAL EXTENSION FOR MODELLING EMPATHIC AGENTS}

nal behaviour simultaneously. Moreover, in general, these proposals are based on ad hoc specifications and do not take advantage of well-known agent languages. In this section, we present an empathic agent model based on AgentSpeak, which is one of the best known agent-oriented programming languages. Our model extends the syntax, semantics, and the reasoning cycle of AgentSpeak to support the development of agents that are capable of performing empathic interactions. We propose a new inference model that allows emotions and empathy to have an effect on the practical reasoning of the agent. Emotions and empathy will be involved in both the deliberation process when the agent is selecting an intention to commit and in the means-end reasoning process when the agent is deciding on the plan to follow. To do that, we have extended the GenI $A^{3}$ architecture with a new syntax and semantics to facilitate the emergence of empathy in software agents. Our approach includes the three fundamental processes identified in the literature on empathic agents discussed in Section 5.2: perception, empathic appraisal, and empathic regulation. The perception process is an intrinsic part of AgentSpeak. However, we have added a new process to differentiate empathic affective events from other affective events, the event classification process. This process consists of two phases. In the first phase, an event is perceived following the process described in [25]. In the second phase, the event is evaluated to determine whether or not it is an affective event. This second phase defines the agent's cognitive ability to maintain a sense of self as distinct from the target agent to elicit a target-oriented empathic emotion. This cognitive ability is simulated using two different appraisal processes: one for empathic events and the other for non-empathic affective events. On the other hand, empathic appraisal is composed of a self-projection appraisal, which evaluates the event using the agent's own beliefs, concerns, and affective memory. Finally, the empathic regulation process adapts the empathic emotion to the agent's affective characteristics (e.g., mood or personality) and the knowledge that the agent has about the target (e.g., affective link or trust level).

The rest of this section is organized as follows. Section 5.3 introduces an example scenario. In Section 5.3, a new configuration for an empathic agent is proposed. Section 5.3 presents the new inference model. Sections 5.3 and 5.3 introduce an extension of AgentSpeak syntax to allow the definition 


\subsection{FORMALIZATION OF THE AGENTSPEAK EXTENSION TO DEVELOP EMPATHIC AGENTS}

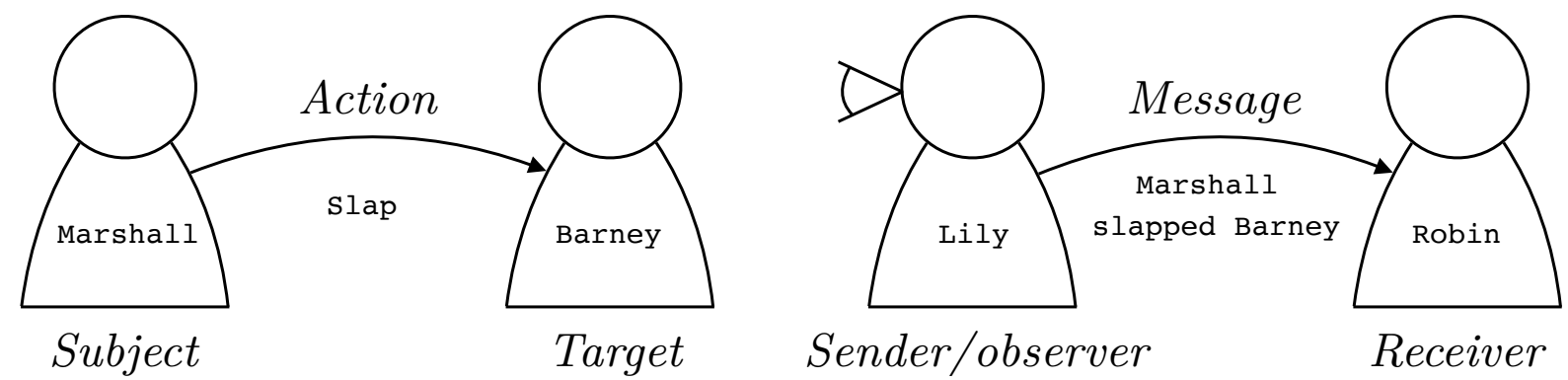

Figure 5.1: Types of agents in a social interaction

of empathic agents. Finally, Section 5.3 describes the set of semantic rules that support the definition of the new inference model.

\section{A simple example}

From the theories of empathy previously cited, it can be assumed that, for empathy to occur, there must be at least two actors: one who suffers in a situation (the target) and another who perceives the situation and reacts empathetically (the observer or empathic agent). To make the reading more convenient, we illustrate the problem with an example. Let us assume a more general scenario with four actors: Marshall, Lily, Barney, and Robin. At one point in time Marshall slaps Barney in the face. This event causes Barney to become sad. Lily, who is in the same room, sees the entire scene and empathizes with Barney and feels sorry for what has happened to him. Later, Lily calls Robin to tell her what happened to Barney. Robin imagines Barny's situation and also feels sad for him.

In this environment, there are basically two interactions: first, Marshall performs an action addressed to Barney; second, Lily perceives what has happened and performs an act of communication to inform Robin (see Figure 5.1). Different types of agents can be identified in these interactions. On the one hand, there are two agents that are involved in the action: Marshall, the agent that performs the action, henceforth known as the subject agent; and Barney, the agent that receives the consequences of the action, henceforth known as the target agent. On the other hand, in the environment there is a third agent, Lily, who perceives the action, henceforth known as the observer agent. 


\section{CHAPTER 5. A FORMAL EXTENSION FOR MODELLING EMPATHIC AGENTS}

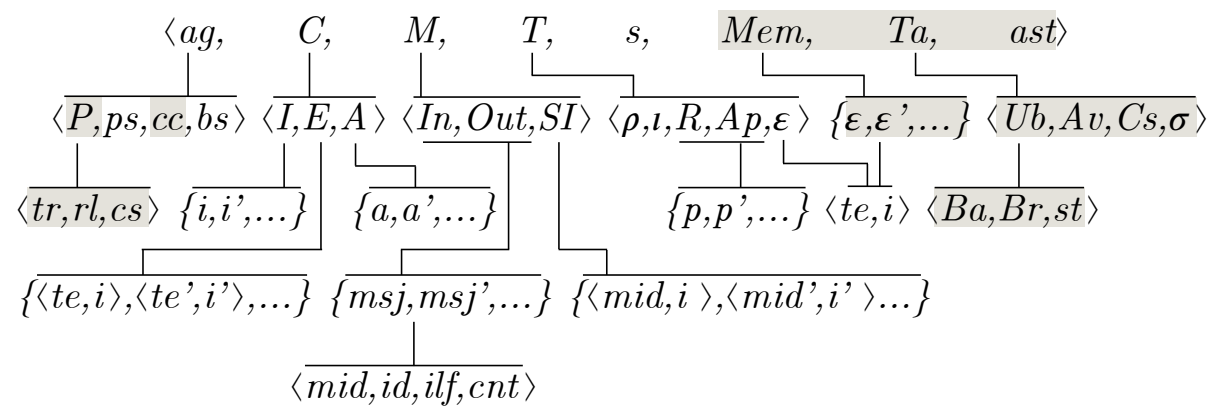

(a) Agent's configuration in $G e n I A^{3}[6]$.

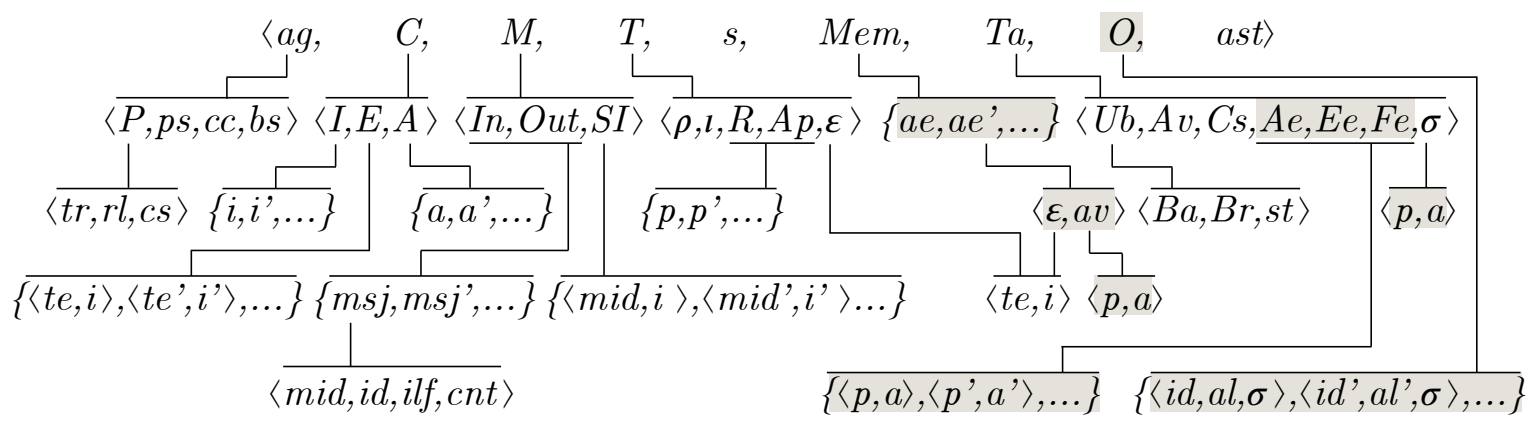

(b) New configuration of an AgentSpeak agent including empathy. New components are shaded.

Figure 5.2: GenIA ${ }^{3}$ configuration.

Finally, there is the communicative act in which two types of agents can be identified: Lily, the observer, who sends a message describing the action, and Robin who receives the message. According to Agent Communication Language (ACL) terminology [160], Lily will be identified as the sender agent and Robin will be identified as the receiver agent. Both agents, the observer and the receiver agents know what has happened to Barney and react to this information with an empathetic response. Therefore, in our model, we consider that both the observer and the receiver agents, are empathic agents.

\section{Formalization of an empathic agent configuration}

In [179], the agent-oriented language AgentSpeak semantics is defined using an operational semantics formalism. This operational semantics defines the structure and the configuration of the agent program and the transitions derived from its internal reasoning. In this section, we extend this operational 


\subsection{FORMALIZATION OF THE AGENTSPEAK EXTENSION TO DEVELOP EMPATHIC AGENTS}

semantics to define the new AgentSpeak configuration for empathic agents.

Figure 5.2a shows the original configuration of an agent in the architecture $G e n I A^{3}$. The shaded attributes are the ones that $G e n I A^{3}$ added to the original configuration proposed for AgentSpeak in [24]. We have extended this configuration to add information that is relevant to the development of empathic agents. The new attributes are shaded in Figure 5.2b. In this new configuration, an agent is defined by a tuple $\langle a g, C, M, T, s, M e m, T a, O$, ast $\rangle$, where:

- $a g$ is the set of attributes that constitute the agent defined by the tuple $\langle P, p s, c c, b s\rangle$, where:

- $P$ is the agent's personality represented by the tuple $\langle t r, r l, c s\rangle$, where:

- $t r$ is a set of personality traits. Each personality trait is a value that is bounded between $[0,1]$ representing the level that the agent has of that personality trait. For example, when using the OCEAN model, five personality traits are defined, one for each component of the OCEAN model: Openness, Conscientiousness, Extraversion, Agreeableness, and Neuroticism.

- $r l$ is a number that is bounded between $[0,1]$ representing the agent's rationality level. The higher the rationality level, the higher the priority of plans activated in a rational BDI cycle, making the agent more rational. However, the lower the rationality level, the higher the priority of plans activated by the affective cycle, making the agent more emotional.

- $c s$ is the agent's set of coping strategies. These coping strategies relate affective states and beliefs to a set of intentions that will be included in the agent's agenda.

- ps and bs represent the set of plans and the set of beliefs of the agent, respectively.

- $c c$ is the set of concerns of the agent that represents the motivations, standards, ideals and/or interests of the agent. 


\section{CHAPTER 5. A FORMAL EXTENSION FOR MODELLING EMPATHIC AGENTS}

- $C$ is the current circumstance represented by a tuple composed of: $I$, which is the set of intentions $\left\{i, i^{\prime}, \cdots\right\} ; E$, which is a set of events composed of a set of tuples 〈triggering event te, intention $i\rangle$; and $A$, which is a set of actions $\left\{a, a^{\prime}, \cdots\right\}$.

- $M$ are the communication parameters represented by the tuple $\langle$ In, Out, SI $\rangle$, where $S I$ is a set of suspended intentions composed by a set of tuples $\langle$ message identifier mid, intention $i\rangle$ and $I n$ and Out represent the list of input and output messages, respectively. Each message $m s j$ is composed of the message identifier mid, the identifier of the agent which sent the message $i d$ (i.e., the sender agent), the illocutionary force $i l f$, and the message content cnt.

- $T$ is the temporary information of the current rational cycle consisting of a tuple containing: an applicable plan $\rho$, a particular intention $\iota$, the sets of relevant plans $R$, the set of applicable $A p$ plans composed of a set of plans $\left\{p, p^{\prime}, \cdots\right\}$, and the event $\varepsilon$ that triggered the rational cycle represented by a tuple 〈triggering event te, intention $i\rangle$.

$-s$ is the current step of the rational cycle where:

$$
\begin{array}{r}
s \in\{\text { ProcMsg, SelEv, RelPl, AppPl, SelAppPl } \\
\text { AddIm, SelInt, ExcInt, CrlInt }\}
\end{array}
$$

- Mem is the affective memory that, in the original GenIA ${ }^{3}$ architecture (Figure 5.2a), consists of a set of events. In our proposal (Figure 5.2b), it consists of a set of affective events $a e$. An affective event $a e$ is defined as a tuple $\langle$ event $\varepsilon$, affective value $a v\rangle$, where the affective value $a v$ is an attribute that represents the emotion that the event $\varepsilon$ produced in the agent.

- Ta represents the temporal information of the affective cycle. In the original design of GenIA $^{3}$ (Figure 5.2a), it is composed of: $U b$, which is a tuple containing the set of beliefs that are going to be added to the belief base $B a$, the set of beliefs that are going to be removed from the 


\subsection{FORMALIZATION OF THE AGENTSPEAK EXTENSION TO DEVELOP EMPATHIC AGENTS}

belief base $B r$, and the identifier of the step st of the cycle in which the beliefs are going to be added or removed; $A v$, which is the set of appraisal variables; $C s$, which is the set of coping strategies to be executed; $A e$, which is the set of emotions that can be elicited by the appraisal process; Ee, which is the set of empathic emotions that can be triggered by the empathic appraisal process; Fe, the final emotion (or emotions) resulting from the emotion selection process; and $\sigma$, which represents the current mood of the agent. The emotions contained in $F e$ will be the ones considered as active in the affective cycle. Emotions and mood are represented by a tuple $\langle p, a\rangle$, where $p$ and $a$ are the values of Pleasure and Arousal, respectively.

- $O$ is a new component which is added to represent the information that the empathic agent knows about other agents in the environment. In the current design, this information is composed of a set of tuples each of which corresponds to one agent in the environment. Each tuple contains the agent identifier $i d$, the affective link al that the empathic agent has with the agent identified by $i d$, and the $i d$ agent's mood $\sigma$. This tuple can be extended in the future to contain more knowledge about agents in the environment, such as their concerns, goals, beliefs, or trust level. The affective link is a numerical value between $[-1,1]$ that indicates the affective link between both agents. The greater the affective link value, the greater the relationship between the agents. Negative affective links indicate enmity between the agents. The affective link can be modified due to the interactions between the agents.

- ast is the current step of the affective cycle, where:

$$
\begin{array}{r}
\text { ast } \in\{\text { EvClass, Appr, EmphAppr, EmReg, EmphReg, } \\
\text { EmSel, AffAd, SelCs, Cope }\}
\end{array}
$$

\section{A new rational cycle for an empathic BDI agent}

The formal definition of the AgentSpeak reasoning cycle was presented in [24]. We have extended that original AgentSpeak reasoning cycle to incorpo- 


\section{CHAPTER 5. A FORMAL EXTENSION FOR MODELLING EMPATHIC AGENTS}

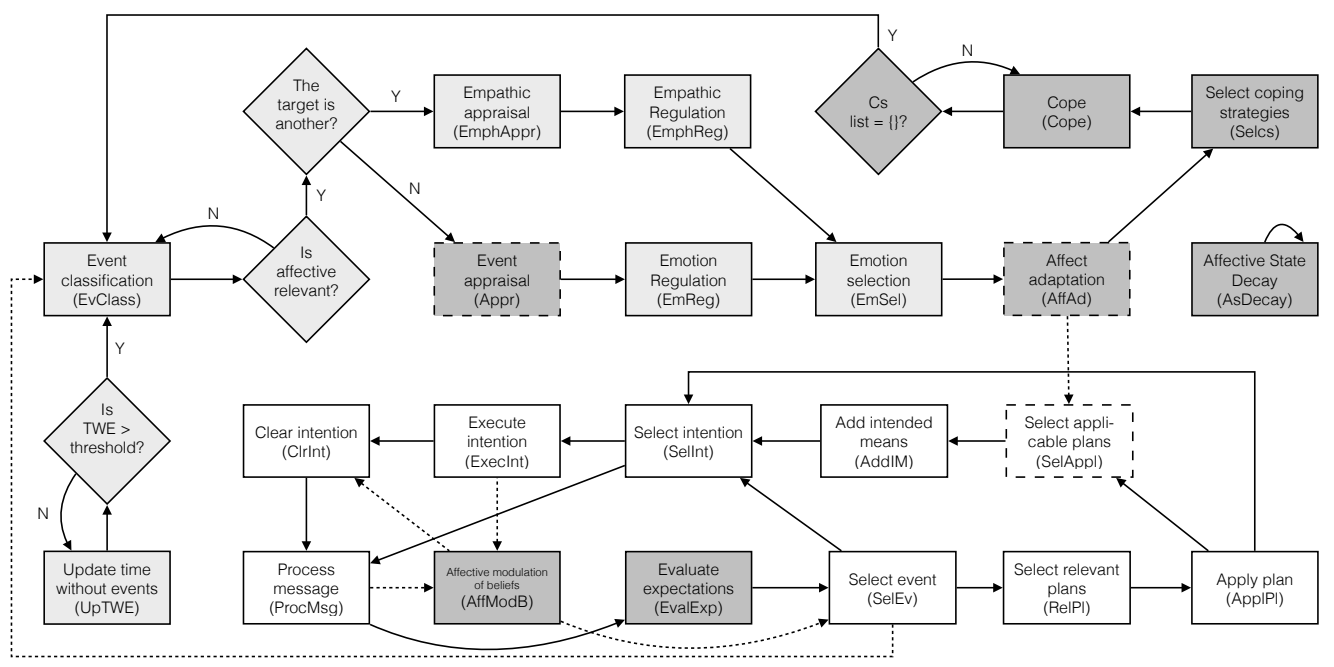

Figure 5.3: New configuration of both affective and rational cycles.

rate affective characteristics. This new reasoning cycle is represented in Figure 5.3: white boxes represent the original processes proposed for an AgentSpeak agent; dark gray shaded boxes are the original processes of the GenIA ${ }^{3}$ architecture; dashed lines indicate processes that have been redesigned to include our extension; light gray shaded boxes represent the new processes of our extension. First, in the process message (ProcMsg) step, all of the pending messages received by the agent are processed; then, the select event (SelEv) step selects one event to process taking into account all of the perceptions, messages, and stacked events. Next, the relevant plans (RelPl) step retrieves all of the relevant plans considering the selected event; these plans are used to generate a list of applicable plans in the apply plans (AppPI) step. From this list, one applicable plan is selected in the select applicable plan (SelAppl) step. Then, in the add intended means (AddIM) step, all of the intended means are added to the set of intentions; the select intention (SelInt) step selects one intention to execute from the set. The selected intention is executed in the execute intention (ExcInt) step. Finally, the executed intention is cleared from the set of intentions in the clear intention (ClrInt) step.

This reasoning cycle was later modified by the $G e n I A^{3}$ architecture to allow the development of affective agents. GenI $A^{3}$ introduced a new affective cycle that consist of five steps: the event appraisal (Appr) step in which 


\subsection{FORMALIZATION OF THE AGENTSPEAK EXTENSION TO DEVELOP EMPATHIC AGENTS}

the selected event is appraised; the affect adaptation (AffAd) step in which the agent's mood is modified to adapt the mood according to the result of the appraisal; the select coping strategies step in which the coping strategies are selected according to the agent's mood; and the cope (Cope) step, in which the coping strategies are executed. In addition, GenI $A^{3}$ incorporates an affective state decay process (AsDecay) that continuously shifts the mood toward an equilibrium state to simulate real mood change in humans. The rate at which the mood moves to the equilibrium state can be set by the user. Finally, GenIA $A^{3}$ modified the rational cycle to add two steps: the affective modulation of beliefs (AffModB) step in which beliefs are modified according to the agent's mood; and the evaluate expectations (EvalExp) step, which includes the possibility to add temporal expectations to agents [217].

We have redesigned the $G e n I A^{3}$ architecture by adding new processes to enable the development of empathic agents. Figure 5.3 shows the new steps of both affective and rational cycles in light gray. We have introduced five new steps to the affective cycle. The first step is the event classification (EvClass) step in which an event is analyzed to determine if it is an affective event and if it can produce empathy. First of all, the process analyzes whether the event can elicit an emotion, classifying the event as affective or non-affective. Second, the process analyzes whether the affective event is directed to another agent, classifying the event as an affective empathic event or the event is directed to the agent itself, in which case, the agent will consider this event as a non-empathic affective event. This second part of the event classification (EvClass) step allows the agent to maintain a sense of self as distinct from the target. If the selected event is a non-empathic affective event, in the next step, the emotion regulation (EmReg) step adjusts the emotions produced by the appraisal according to the affective characteristics of the agent. Otherwise, if the selected event is an empathic event, the empathic appraisal (EmphAppr) process will determine a set of possible empathic emotions based on the perceptions and knowledge that the empathic agent has about the target agent. Then, the empathic regulation (EmphReg) process adapts these empathic emotions to the affective characteristics of the agent taking into account the knowledge that the empathic agent has about the target agent. Finally, in the emotion selection (EmSel) process, the final 
emotion to be triggered by the empathic agent is selected. This emotion will modify the agent's mood, and will affect the selection of the applicable plan and the selection of coping strategies. In addition, we have proposed a new process named update time without events (UpTWE), which allows the development of affective behaviors such as boredom or sleepiness when the agent is not receiving events.

\section{Extending the AgentSpeak language to identify the actors of an empathic interaction}

In AgentSpeak, an agent is defined as a set of beliefs and a set of plans. Beliefs define the knowledge that the agent has about the environment. The set of beliefs of the agent is known as the belief base. The knowledge contained in the belief base may not necessarily be complete or accurate, since the environment may be very large and may experience changes that the agent has not perceived. On the other hand, plans contain basic actions that the agent can perform to change its environment. Plans are composed of a triggering event, a context, and a set of sequential instructions that may include: updates to the belief base, actions, or goals. Triggering events refer to the addition or deletion of beliefs or goals. In AgentSpeak, both beliefs and goals are defined as atomic formulas [179]. An atomic formula representing a belief or a goal is composed of a predicate and a set (possibly empty) of $n$ terms of a first order logic:

$$
\text { predicate }\left(\text { term }_{1}, \text { term }_{2}, \cdots, \text { term }_{n}\right)
$$

For example, the triggering event time(cloudy) is composed of the predicate 'time' and the term 'cloudy'.

In [224], the concept of annotations was introduced to the AgentSpeak language providing agents with the ability to identify the source of the triggering event. This concept of annotation was later reformulated by [24] to allow annotations to express properties associated with events and beliefs. The proposed syntax for defining beliefs, goals, and triggering events using annotations in AgentSpeak is: 


\subsection{FORMALIZATION OF THE AGENTSPEAK EXTENSION TO DEVELOP EMPATHIC AGENTS}

$$
\text { predicate }\left(\text { term }_{1}, \operatorname{term}_{2}, \cdots, \text { term }_{n}\right)\left[\mathrm{a}_{1}, \mathrm{a}_{2}, \cdots, \mathrm{a}_{m}\right]
$$

where each $a_{i}$ represents the $i$ th annotation defined as:

$$
\mathrm{a}_{i}=\operatorname{functor}_{i}\left(\operatorname{term}_{i, 1}^{\prime}, \operatorname{term}_{i, 2}^{\prime}, \cdots, \operatorname{term}_{i, n}^{\prime}\right)
$$

where an atom (called functor) is followed by a number of terms (called arguments). term ${ }_{i, j}^{\prime}$ is the $j$ th term of the annotation $\mathrm{a}_{i}$.

This extension of the language provided more expressiveness to AgentSpeak allowing different types of properties to be defined. For example, we can add one annotation to an event to represent the sources of the event. The keyword that we use to represent this annotation is "source". For example, in the triggering event:

$$
\text { time (cloudy) [source(Marshall)] }
$$

the annotation source(Marshall) indicates that the source of the triggering event is the agent Marshall.

In general, there are three possible sources for an event:

- perceptions, which represent the information that the agent perceives from its environment. Perceptions are represented by the annotation source (percept).

- mental notes, which represent beliefs that the agent acquires or deduces by itself, such as memories or changes in the agent's state. The mental notes are expressed through the annotation source(self).

- communications, which is the information that comes from another agent of the system as a consequence of a communication act. Communications are represented by the annotation source(agent_id) where agent_id is the identifier of the sender agent that originates the communication act. 


\section{CHAPTER 5. A FORMAL EXTENSION FOR MODELLING EMPATHIC AGENTS}

In our proposal, we extend the language proposed in $G e n I A^{3}$ with new annotations that allow the events with relevant information to be contextualized for empathy simulation. As we discussed in Section 5.2, the empathic regulation process is affected by a set factors that include interpersonal factors related to the target agent such as the affective link, concerns, goals, similarity, or trust level. Therefore, to elicit an empathic emotion, it is necessary to identify the target agent when an event occurs. This information can be implicit in the semantics of the event, in which case the agent may deduce the target of the action through an inference process, or it can be explicitly incorporated into the syntax of the triggering event. We have used this second approach because it simplifies the agent's programming by easily identifying the subject and the target of an event.

We propose an extension of the expressiveness of AgentSpeak to describe the participants in the action to which the event refers. By adding new elements it will be possible to know the agents that participate in the event when an event is received. We have extended the representation of a triggering event to explicitly include the agents involved in an action (i.e., subject and target agents) without modifying the original syntax of the atomic formula of AgentSpeak. This has been achieved using two annotations to identify the agents participating in the action represented by the triggering event: the subject annotation, which identifies the subject agent that performs the action; and the target annotation, which identifies the target agent receiving the consequences of the action. Following this definition a triggering event will be represented by the structure:

$$
\begin{aligned}
\operatorname{predicate} & \left(\operatorname{term}_{1}, \operatorname{term}_{2}, \cdots, \operatorname{term}_{n}\right) \\
& {\left[\text { subject }(\text { subject_id }), \operatorname{target}(\text { target_id }), \mathrm{a}_{3}, \mathrm{a}_{4}, \cdots, \mathrm{a}_{n}\right] }
\end{aligned}
$$

Where subject_id and target_id are the identifiers of the subject agent and the target agent, respectively. Note that the order in which the annotations are defined is not relevant since the annotations can be written in any order. By adding these new annotations, it is now possible to identify the agents involved in any action. Based on the example of Section 5.3, we can use 


\subsection{FORMALIZATION OF THE AGENTSPEAK EXTENSION TO DEVELOP EMPATHIC AGENTS}

the following expression to specify the triggering event "Marshall has slapped Barney":

$$
\text { slap [subject (Marshall), target (Barney)] }
$$

This event has a predicate "slap" and two annotations: subject (Marshall) and target (Barney). Moreover, we propose adding an optional annotation to include the value of the interaction. The interaction value of a triggering event is an optional number that is associated with the triggering event in the range of $[-1,1]$, which identifies if the interaction is good (positive interaction value), bad (negative interaction value), or neutral (interaction value equals 0 ) for the agent that receives the action. The interaction value is used to update the affective link between the agents. A positive interaction value indicates that the interaction has a positive effect on the affective state of the target agent, improving the affective link that the target agent has with the subject agent. A negative interaction value has a negative impact in the agent's affective state and decreases the affective link. An interaction value equal to 0 denotes that the interaction has a neutral effect on the affective state of the agent. Therefore, it has no effect on the affective link.

We are going to define the functions for calculating the values associated with the annotations introduced in our proposal. These functions will be used in Section 5.3 to formalize the new internal processes of the affective cycle of an agent. Let us define te $e_{\text {annots }}$ as the set of annotations associated to the triggering event te. The function to obtain the subject agent of a triggering event te is defined as:

(5.7) getSubject $(t e)= \begin{cases}\text { term }_{i}, & \text { if } \exists a_{i} \in \text { te }_{\text {annots }} \mid \text { functor }_{i}=\text { 'subject' } \\ & \text { and term } \text { ter }_{i} \neq a g_{i} i d \\ \text { self, } & \text { if } \exists a_{i} \in \text { te } e_{\text {annots }} \mid \text { functor }_{i}=\text { 'subject' } \\ & \text { and term } \text { fun_ }_{i}=a g_{-} \text {' } \\ \text { null, } & \text { otherwise }\end{cases}$

where $a_{i}$ is the $i$ th annotation of the set of annotations te $e_{\text {annots }}$, functor is the functor of the annotation $a_{i}$, term $_{i}$ is the term of the annotation $a_{i}$, 
'subject' is a keyword that identifies the subject annotation, and $a g \_i d$ is the agent's identifier. If term $_{i}$ is equal to the $a g \_i d$, it indicates that the triggering event comes from the agent itself. If $\operatorname{term}_{i}$ is not the ag_id, it indicates that the triggering event comes from another agent. Finally, if there is no 'subject' annotation, it indicates that this triggering event comes from an unknown subject.

Similarly, the target of a triggering event te is obtained through the function:

$$
\text { getTarget }(t e)= \begin{cases}\text { term }_{i}, & \text { if } \exists a_{i} \in \text { te }_{\text {annots }} \mid \text { functor }_{i}=\text { 'target' } \\ \text { null, } & \text { otherwise }\end{cases}
$$

where 'target' is a keyword that identifies the target agent annotation.

Finally, to obtain the interaction value of the triggering event te, we define the function:

$$
\text { getInteractionValue }(t e)= \begin{cases}\text { term }_{i}, & \text { if } \exists a_{i} \in t e_{\text {annots }} \mid \\ 0, & \text { functor }_{i}=\text { 'InteractionValue' } \\ 0, & \text { otherwise }\end{cases}
$$

where 'InteractionValue' is a keyword that identifies the annotation that contains the interaction value.

\section{Extending the AgentSpeak agent configuration}

The syntax of AgentSpeak was presented in [25] using the EBNF (Extended Backus-Naur Form) notation. This syntax was later extended by the GenIA ${ }^{3}$ architecture to allow the development of affective agents $[6,216,217]$. In the original EBNF syntax of $G e n I A^{3}$, an agent (agent) is defined by the set of initial beliefs (init_beliefs), concerns (concerns), personality (personality), initial goals (init_goals), and plans (plans). We have extended this syntax to incorporate some new attributes to the agent configuration. Figure 5.4 shows the extension of the GenIA ${ }^{3}$ agent's EBNF syntax. We have added 


\subsection{FORMALIZATION OF THE AGENTSPEAK EXTENSION TO DEVELOP EMPATHIC AGENTS}

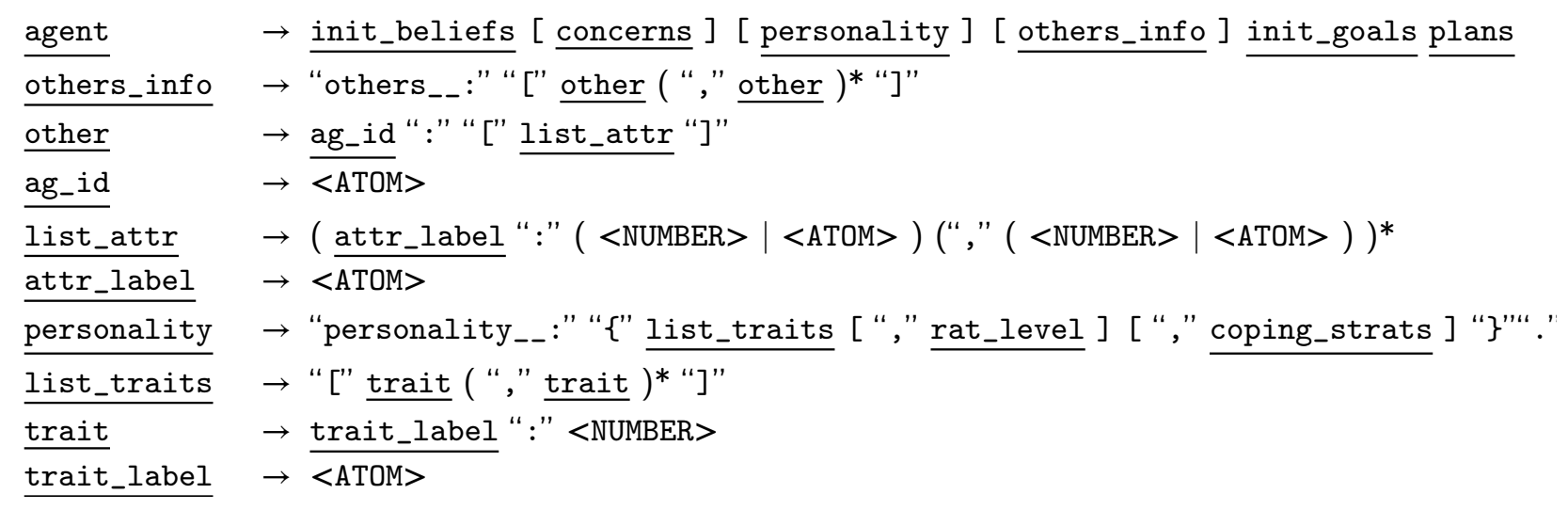

Figure 5.4: Simplified extension of the agent's syntax including the new extension of GenIA ${ }^{3}$.

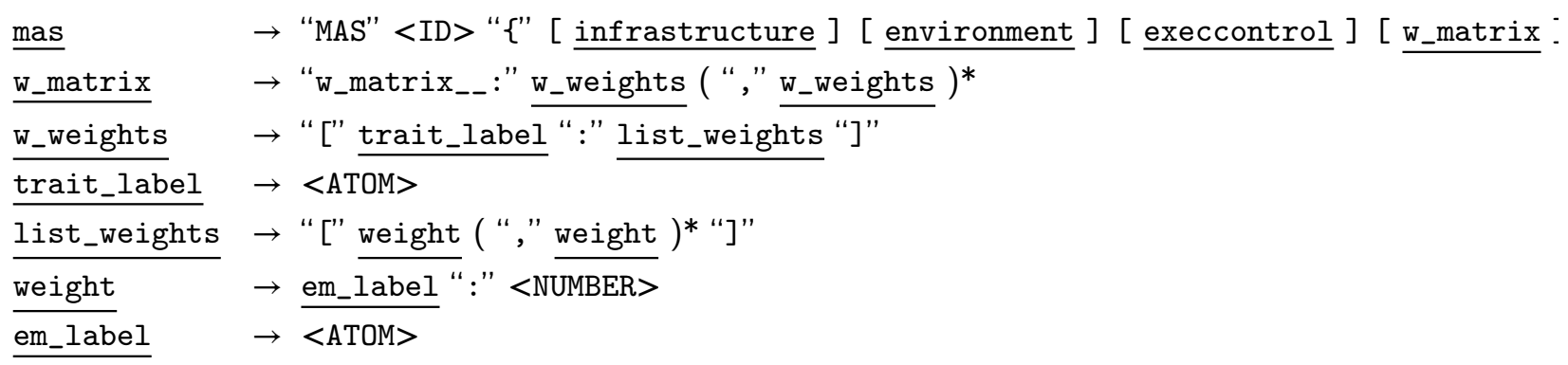

Figure 5.5: Simplified extension of the MAS project syntax including the new extension of GenIA ${ }^{3}$.

a new attribute to represent the knowledge that the agent has about other agents (others_knowledge). To represent this knowledge, we use a set consisting of the agent's identity (ag_id) and a list of attributes associated with that agent (1ist_attr). The list of attributes is defined as a set of tuples consisting of the attribute label (attr_label) and its value. One of the most important attributes is the affective link that represents the affective proximity or relationship between the agents. Following the example described in Section 5.3, let us assume that agent Marshall has an affective link with agent Barney of -0.5 , an affective link with agent Lily of 0.9 , and has no affective link with agent Robin. To represent this knowledge, the following sentences must be added to agent Marshall's definition:

// Marshall agent 


\section{CHAPTER 5. A FORMAL EXTENSION FOR MODELLING} EMPATHIC AGENTS

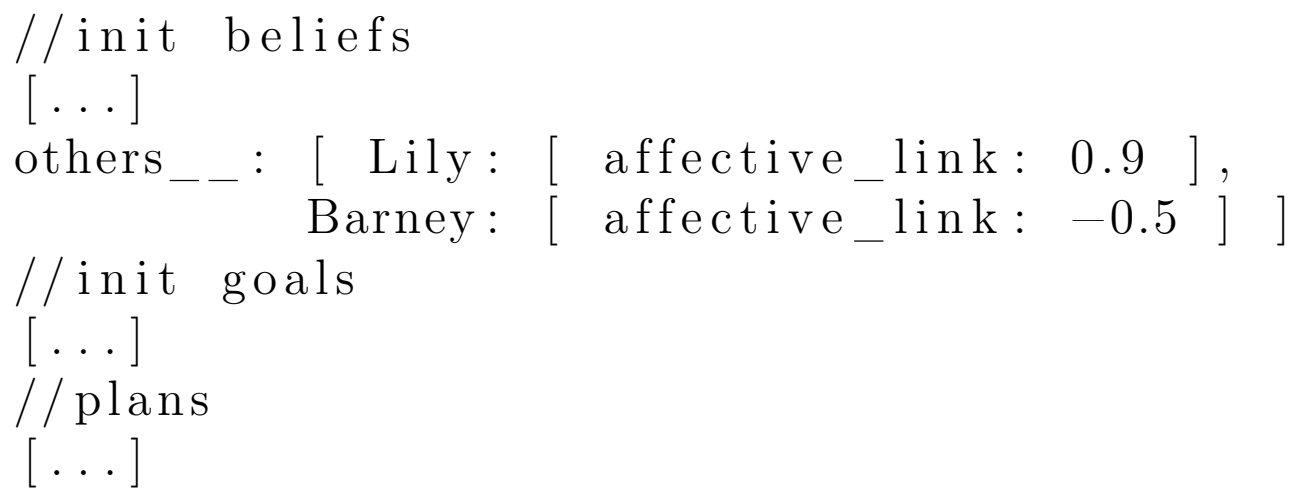

Note that, since agent Marshall does not have any affective link with agent Robin, it is not necessary to mention Robin when defining agent Marshall. Note also that the affective_link is not the only attribute that can be represented. The number of attributes could be increased to represent more information such as trust level or the other agents' goals, concerns, and beliefs.

On the other hand, in GenIA ${ }^{3}$, the agent's personality is defined by the keyword "personality__:" followed by some attributes: the traits (traits), which are defined as a list containing a value for each personality trait; and optionally, the level of rationality (rat_level), which is defined as a numerical value representing how rational the agent is; and the coping strategies (coping_strats), which are defined as a list of plans that will be used by the agent to deal with affective events. We have modified the definition of the personality traits to make it more user friendly. Now, the personality traits are defined using a trait label (trait_label) followed by the numeric value for that trait. For example, let us suppose that the agent Marshall has an Extraversion level of 0.9 and a Neuroticism level of 0.1 . We can express this using the new syntax as follows:

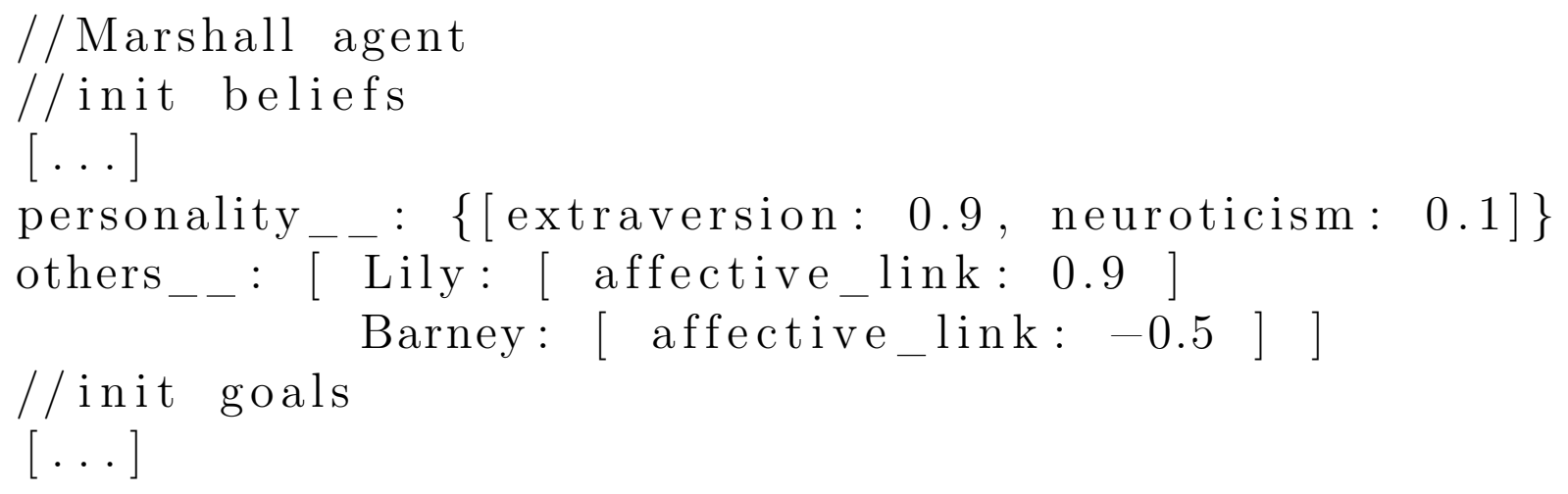




\subsection{FORMALIZATION OF THE AGENTSPEAK EXTENSION TO DEVELOP EMPATHIC AGENTS}

\section{//plans \\ $[\ldots]$}

Finally, we have introduced a new attribute $\omega$ (em_weights) to the multiagent system (MAS) project. $\omega$ represents a correlation matrix between the personality traits $\operatorname{tr} \in P$ and all of the possible emotion types $t \in A e \cup E e$, where $A e$ is the set of emotions that can be elicited by the appraisal process and $E e$ is the set of empathic emotions that can be triggered by the empathic appraisal process. For each pair of values $(t r, t)$, a weight $p$ representing how the personality trait $t r$ influences the emotion type $t$ can be specified, but many of these weights will be zero. Therefore, the set of weights of the emotions can be viewed as the set of correlations between emotions and personality traits: the greater the correlation between the personality trait $t r$ and the emotion type $t$, the greater the value of $p$. For example, considering that the trait of Extraversion $E$ is related to positive emotions and Neuroticism $N$ does not have a very high relation with positive emotions [75], for the Happiness emotion, the Extraversion weight $\left(\omega_{\text {Happiness }, E}\right)$ will be greater than the weight of the Neuroticism for the Happiness emotion $\left(\omega_{\text {Happiness }, N}\right)$.

To define the syntax of the $\omega$ matrix, we have proposed the use of the keyword "w_matrix__:" followed by one or more weights (w_weights). These weights are defined as a label that identifies the personality trait (trait_label) followed by a list of weights (list_weights). This list of weights is composed of one or more emotion labels (em_label) followed by a number that represents the correlation between that personality trait and that emotion. For instance, let us consider the example proposed in [221] in which a personality / emotion correlation matrix is defined based on the variables Extraversion and Neuroticism and the emotions Anger and Sadness. Table 5.1 shows the correlation matrix. The definition of the $\omega$ matrix based on our proposed syntax is:

$$
\begin{aligned}
& \text { w_matrix__ : [ extraversion: [Anger: 0.5, Sadness: 0.6], } \\
& \text { neuroticism: [Anger: 0.8, Sadness: } 0.7 \text { ]] }
\end{aligned}
$$

Through our extension of AgentSpeak, the programmer can clearly define both the personality of the agent and the knowledge that the agent has about other agents. On the one hand, instead of establishing one threshold for each 


\section{CHAPTER 5. A FORMAL EXTENSION FOR MODELLING EMPATHIC AGENTS}

Table 5.1: Example of a personality / emotion correlation matrix.

\begin{tabular}{lcc}
\hline & Extraversion & Neuroticism \\
\hline Anger & 0.5 & 0.8 \\
Sadness & 0.6 & 0.7 \\
\hline
\end{tabular}

emotion as seen in previous approaches, our model allows the relationship between different personality traits and emotions to be defined using the correlation matrix $\omega$. Therefore, just by varying the personality traits we are defining agents with different affective behaviors as humans. On the other hand, in our proposal, each agent can have its own affective link matrix, thus allowing the implementation of asymmetric relationships between agents. For instance, based on their previous interactions, Marshall has a negative affective link with Barney, while Barney feels a positive affective link with Marshall. This difference in the affective links between both agents represents what happens in human societies in real life. In addition, the affective link between two agents can dynamically vary according to the interaction value and the emotion generated when the agents interact with each other. This allows the simulation of affective behavior in long-term interactions between agents.

Definitions of non-terminal syntactic symbols that have not been described in this section are detailed in $[25,6]$.

\section{Formalization of the semantic rules of the new affective cycle}

We are going to formalize the transitions between the different states of the new affective cycle presented in Section 5.3 (Figure 5.3) using the same operational semantics used to define the original rational cycle of AgentSpeak ([224]). This operational semantics is described in [171] and consists of a set of rules that define the transitions between different configurations of an AgentSpeak agent. To make readability more convenient, we have adopted the syntax proposed in [224]. Following this syntax, if $a g$ is the set of attributes that define an agent, we will refer to the personality component $P$ 


\subsection{FORMALIZATION OF THE AGENTSPEAK EXTENSION TO DEVELOP EMPATHIC AGENTS}

of $a g$ as $a g_{P}$. We will also refer to the empathic agent whose affective cycle we are describing as empathic agent. In addition, we will use the label emph_ag_id when referring to the empathic agent identifier.

\section{Event classification}

The event classification (EvClass) step evaluates the triggering event te component of the current event $T_{\varepsilon}$ to determine whether the event is affectively relevant or not using the function:

$$
\operatorname{affRelEv}(t e)= \begin{cases}\text { True, } & \text { if } \exists a_{i} \in \text { te } e_{\text {annots }} \mid \\ \text { False, } & \text { otherwise }\end{cases}
$$

where $t e$ is the triggering event, te $e_{a n n o t s}$ is the set of annotations of $t e, a_{i}$ is the $i$ th annotation of the set of annotations $t e_{\text {annots }}$, and functor $r_{i}$ represents the functor of the annotation $a_{i}$.

Furthermore, this step also performs the dissociation between events that should be evaluated at an affective level and those that should be evaluated at an empathic level allowing the distinction between the self from the target. As discussed in Section 5.2, this distinction between self and target is essential in the development of empathy since it allows an agent to differentiate its own emotions from emotions produced by the perception of others. For an event, we have designed four transition rules in this step. The first rule (EvClass 1 ) is applied to events that are not affectively relevant and either have nothing to do with the empathic agent (e.g., the target are other agents) or events whose target is the empathic agent but without a subject agent (e.g., environmental events):

$\left(\mathrm{EvClass}_{1}\right)$

$$
\frac{\neg \operatorname{affRelEv}(t e) \wedge\left(t g \neq e m p h \_a g \_i d \vee\left(t g=e m p h \_a g \_i d \wedge s b j \in\{\text { null, self }\}\right)\right)}{\langle a g, C, M, T, s, M e m, T a, O, \text { EvClass }\rangle \rightarrow\left\langle a g, C, M, T^{\prime}, s, M e m, T a, O, \text { EvClass }\right\rangle}
$$




$$
\text { where: } \begin{aligned}
& T_{\varepsilon}=\langle t e, i\rangle \\
t g & =\operatorname{getTarget}(t e) \\
& s b j=\operatorname{getSubject}(t e)
\end{aligned}
$$

If this rule is evaluated as true, the empathic agent will remain in the state EvClass waiting for a new event. For example, let us assume that Lily is the empathic agent. At a certain point, in time Lily perceives the event time(cloudy). The function affRelEv evaluates the event obtaining the value false as a result because this event is not relevant for the affective cycle. The function getTarget also evaluates the event and returns the value null since there is no annotation indicating a target agent at the event. Therefore, the event does not have any affective impact on Lily, and the affective cycle is not started. Lily will remain in the event classification step waiting for a new event.

The second rule $\left(\mathrm{EvClass}_{2}\right)$ deals with events that are not affectively relevant but that have some level of social interaction between the empathic agent and the subject agent.

$\left(\mathrm{EvClass}_{2}\right)$

$$
\begin{aligned}
& \neg \operatorname{affRelEv}(t e) \wedge t g=e m p h \_a g \_i d \wedge s b j \notin\{\mathrm{null}, \mathrm{self}\} \\
& \overline{\langle a g, C, M, T, s, \mathrm{Mem}, \mathrm{Ta}, \mathrm{O}, \text { EvClass }\rangle \rightarrow\left\langle a g, C, M, T, s, \mathrm{Mem}, \mathrm{Ta}, O^{\prime}, \text { EvClass }\right\rangle} \\
& \text { where: } T_{\varepsilon}=\langle t e, i\rangle \\
& t g=\text { getTarget }(t e) \\
& s b j=\text { getSubject }(t e) \\
& i v=\text { getInteractionValue }(t e) \\
& O_{a l}^{\prime}=\text { updateAl }\left(O_{a l}, i v\right)
\end{aligned}
$$

When this rule is evaluated as true, the affective link that the empathic agent has with the subject agent is updated by the function updateAl. Continuing with the example, suppose that Lily (the empathic agent) has an affective link of 0.5 with Marshall. At a certain point in time, Marshall says hello to Lily by the event: 


\subsection{FORMALIZATION OF THE AGENTSPEAK EXTENSION TO DEVELOP EMPATHIC AGENTS}

(Example 1)

hello[subject (Marshall), target(Lily), interaction_value (0.2)]

In this event, Marshall is the subject agent. As a result of this interaction, Lily will increase her affective link with Marshall by using the interaction value 0.2 . As in the previous rule, Lily will remain in the event classification step waiting for a new event.

The last two rules refer to events that are affectively relevant and, therefore, will trigger the affective cycle. It is in these rules where the distinction between self and target occurs. This distinction is crucial because it allows an agent to differentiate its own emotions from emotions produced by the perception of others. The EvClass 3 rule is evaluated as true when affective events are directed towards other agents:

$\left(\mathrm{EvClass}_{3}\right)$

$$
\begin{gathered}
\frac{\operatorname{affRelEv}(t e) \wedge t g \notin\left\{e m p h \_a g \_i d, \text { null }\right\}}{\langle a g, C, M, T, s, M e m, T a, O, \text { EvClass }\rangle \rightarrow\left\langle a g, C, M, T, s, M e m, T a, O^{\prime}, \text { EmphAppr }\right\rangle} \\
\text { where: } T_{\varepsilon}=\langle t e, i\rangle \\
t g=\operatorname{getTarget}(t e) \\
i v=\operatorname{getInteractionValue~}(t e) \\
O_{a l}^{\prime}=\operatorname{updateAl}\left(O_{a l}, i v\right)
\end{gathered}
$$

If an event is affectively relevant and the target agent is another agent, the event is considered an empathic event. Therefore, the affective cycle is started, and the empathic appraisal step processes the empathic event. For example, when Lily receives the event:

slap [subject (Marshall), target (Barney),

(Example 2) affective_relevant,interaction_value(-0.5)] 


\section{CHAPTER 5. A FORMAL EXTENSION FOR MODELLING} EMPATHIC AGENTS

$*$

The function affRelEv evaluates the event as affectively relevant and identifies that the target of the triggering event is Barney. Therefore, the affective cycle of agent Lily moves to the appraisal (Appr) step in which the event is evaluated by means of an empathic appraisal. In this appraisal step, Lily infers how Barney feels, and she generates an empathic emotion directed towards Barney.

Finally, the rule EvClass 4 deals with affectively relevant events that are received by the empathic agent:

$\left(\mathrm{EvClass}_{4}\right)$

$$
\begin{aligned}
& \operatorname{affRelEv}(t e) \wedge t g=e m p h \_a g \_i d \\
& \overline{\langle a g, C, M, T, s, \text { Mem, Ta, O, EvClass }\rangle \rightarrow\langle a g, C, M, T, s, \text { Mem, Ta, O, Appr }\rangle} \\
& \text { where: } T_{\varepsilon}=\langle t e, i\rangle \\
& t g=\text { getTarget }(t e)
\end{aligned}
$$

When this rule is evaluated as true, the empathic agent performs a selfappraisal process of the event obtaining an emotion as a result. Following the previous example, when Lily receives the event:

(Example 3) affective_relevant,interaction_value(-0.5)]

this event is directed toward Lily (i.e., Lily is the target agent). Therefore, Lily will evaluate this event through a self-appraisal process in the appraisal (Appr) step.

\section{Appraisal}

In the appraisal step, the values of the appraisal variables $A v$ for the event $\varepsilon$ are determined by the self-appraisal process defined by the function: 


\subsection{FORMALIZATION OF THE AGENTSPEAK EXTENSION TO DEVELOP EMPATHIC AGENTS}

$$
\begin{aligned}
\operatorname{Appraisal} & (\varepsilon, b s, c c, A v, M e m, A p)= \\
& \left\{\left(x_{i d}, x_{v}\right) \mid x_{v}=\operatorname{DeriveAV}\left(x_{i d}, x_{v}, \varepsilon, b s, c c, M e m, A p\right),\right. \\
& \left.\forall\left(x_{i d}, x_{v}\right) \in A v\right\}
\end{aligned}
$$

where $b s$ are the agent's beliefs, $c c$ are the agent's concerns, $A v$ is a list of tuples (identifier, value) containing the set of appraisal variables, Mem is the affective memory, $A p$ are the applicable plans, $x_{i d}$ is the identifier of an appraisal variable, $x_{v}$ is the value of the appraisal variable, and DeriveAV is a function that returns the value of each appraisal variable. Note that this function calculates a different value for each appraisal variable. To determine the derived emotions from the appraisal variables, we have defined the DeriveEm function as:

$$
\operatorname{DeriveEm}(A v)=A e \mid A v \mapsto A e
$$

where $A v$ represents the set of appraisal variables and $A e$ represents the set of appraised emotions defined as a set of tuples $\left\{\langle p, a\rangle,\left\langle p^{\prime}, a^{\prime}\right\rangle, \cdots\right\}$ where $p$ and $a$ represent the Pleasure and Arousal values associated with the emotion. Therefore, this function establishes a mapping between the set of appraisal variables and emotions. There are different proposals for performing this mapping. In our approach, we have used the model proposed in [221] where a fuzzy model of appraisal based on the K.R. Scherer appraisal theory [201] is used.

The transition rule for the appraisal step of the affective cycle has been defined as:

$\left(\operatorname{Appr}_{1}\right)$

$$
\begin{gathered}
\frac{\text { True }}{\langle a g, C, M, T, s, M e m, T a, O, \operatorname{Appr}\rangle \rightarrow\left\langle a g, C, M, T, s, M e m, T a^{\prime}, O, \text { EmReg }\right\rangle} \\
\text { where: } T a_{A v}^{\prime}=\operatorname{Appraisal}\left(T_{\varepsilon}, a g_{b s}, a g_{c c}, T a_{A v}, M e m, T_{A p}\right) \\
T a_{A e}^{\prime}=\operatorname{DeriveEm}\left(T a_{A v}^{\prime}\right)
\end{gathered}
$$




\section{CHAPTER 5. A FORMAL EXTENSION FOR MODELLING} EMPATHIC AGENTS

For instance, continuing with the event of Example 3, Lily appraises the slap event eliciting the emotion sadness with high intensity.

Once the appraisal finishes, the next step in the affective cycle is the emotion regulation (EmReg) step.

\section{Emotion regulation}

The emotion regulation step adapts the appraised emotion based on different factors such as the mood or personality of the agent. This regulation process affects the probability and intensity of emotions. For example, if the affective link between two agents is high, the probability and intensity of emotions should be increased. The process of emotion regulation is established by the EmphRegulation function defined as:

$$
\begin{gathered}
\text { EmRegulation }(t r, \omega, \sigma, A e)=\left\{\left\langle p^{\prime}, a^{\prime}\right\rangle \mid\left\langle p^{\prime}, a^{\prime}\right\rangle=\varphi_{1}(t r, \omega, \sigma,\langle p, a\rangle),\right. \\
\forall\langle p, a\rangle \in A e\}
\end{gathered}
$$

where $\operatorname{tr}$ are the personality traits of the agent, $\omega$ is the personality/emotion correlation matrix, $\sigma$ is the agent's current mood, and $A e$ is the set of appraised emotions. Since we define emotions as vectors in a Cartesian space composed of the dimensions of Pleasure and Arousal $\langle p, a\rangle$, the function $\varphi_{1}$ is defined as an operation on vectors (described in more detail in [221]) that adapts the emotion vector, represented by the tuple $\langle p, a\rangle$, using the agent's personality and mood. Thanks to this regulation of the emotion process, each agent can face an event in a different way depending on his/her personality or mood.

The transition rule for this step is defined as follows:

$\left(\mathrm{EmSel}_{1}\right)$

$$
\begin{gathered}
\frac{\text { true }}{\langle a g, C, M, T, s, \text { Mem, Ta, O, EmReg }\rangle \rightarrow\left\langle a g, C, M, T, s, M e m, T a^{\prime}, O, \text { EmSel }\right\rangle} \\
\text { where: } T a_{A e}^{\prime}=\text { EmRegulation }\left(a g_{P}, T a_{\sigma}, T a_{A e}\right)
\end{gathered}
$$




\subsection{FORMALIZATION OF THE AGENTSPEAK EXTENSION TO DEVELOP EMPATHIC AGENTS}

Following the previous example in which Lily appraised the sadness emotion with high intensity, if Lily's personality has a high component of extraversion, she is more prone to positive emotions. Therefore, the emotion regulation process will reduce the intensity of the emotion, producing the emotion sadness with medium intensity.

Once the regulation of the possible emotions has been performed, the next step of the non-empathic affective cycle will be the emotion selection (EmSel) step.

\section{Empathic appraisal}

When the target of an affective event is another agent, the event is appraised by the empathic appraisal process. This empathic appraisal process is a selfprojection appraisal that allows an empathic agent to understand another agent's emotion or situation. Similarly to the previously defined self-appraisal step, the empathic appraisal is defined by the formula:

$$
\begin{aligned}
& \text { EmphAppraisal }(\varepsilon, b s, c c, A v, M e m, A p)= \\
& \qquad\left(x_{i d}, x_{v}\right) \mid x_{v}=\operatorname{DeriveAV}\left(x_{i d}, x_{v}, \varepsilon, b s, c c, M e m, A p\right), \\
& \left.\forall\left(x_{i d}, x_{v}\right) \in A v\right\}
\end{aligned}
$$

where $b s$ is the set of beliefs, $c c$ is the set of concerns, Mem is the affective memory, $A p$ are the applicable plans, $x_{i d}$ is the identifier of an appraisal variable, $x_{v}$ is the value of an appraisal variable, $A v$ is a list of tuples (identifier, value) one for each appraisal variable, and DeriveAV is a function that returns the value of each appraisal variable. Note that the beliefs and concerns may be either those of the empathic agent or those of the target agent. In addition, both the number and type of appraisal variables and the function DeriveAV of the empathic process can be different from the selfappraisal process. Empathic emotions can also be distinct from self-appraisal and are elicited through function:

$$
\operatorname{DeriveEmphEm}(A v)=E e \mid A v \mapsto E e
$$




\section{CHAPTER 5. A FORMAL EXTENSION FOR MODELLING EMPATHIC AGENTS}

where $A v$ represents the set of appraisal variables and $E e$ represents the set of empathic emotions defined as a set of tuples $\left\{\langle p, a\rangle,\left\langle p^{\prime}, a^{\prime}\right\rangle, \cdots\right\}$ where each $p$ and $a$ represent the Pleasure and Arousal values associated with the emotions. Similarly to the DeriveEm function, the DeriveEmphEm function also establishes a mapping between the set of appraisal variables and the empathic emotions.

The transition rule for the empathic appraisal step of the affective cycle has been defined as:

$\left(\right.$ EmphAppr $\left._{1}\right)$

$$
\begin{gathered}
\frac{\text { True }}{\langle a g, C, M, T, s, M e m, T a, O, \text { EmphAppr }\rangle \rightarrow\left\langle a g, C, M, T, s, M e m, T a^{\prime}, O, \text { EmphReg }\right\rangle} \\
\text { where: } T a_{A v}^{\prime}=\text { EmphAppraisal }\left(T_{\varepsilon}, a g_{b s}, a g_{c c}, T a_{A v}, M e m, T_{A p}\right) \\
T a_{E e}^{\prime}=\text { DeriveEmphEm }\left(T a_{A v}\right)
\end{gathered}
$$

For example, when Lily perceives the event described in Example 2, Lily evaluates the event slap through a self-projection appraisal and elicits the emotion sorry for with high intensity towards Barney.

Once the empathic appraisal generates the set of empathic emotions, the empathic regulation (EmphReg) process will adapt the empathic emotions using the mood and personality of the empathic agent.

\section{Empathic regulation}

The empathy regulation step adapts the empathic emotion according to different factors such as the mood $T a_{\sigma}$ or personality $a g_{P}$ of the agent and the affective link $O_{a l}$ between the empathic agent and the target agent. This regulation process allows the empathic response to be personalized to different target agents. For example, if the affective link between the empathic agent and the target agent is high, the probability and intensity of the empathic emotions will be higher. The process of empathic regulation is established by the EmphRegulation function defined as: 


\subsection{FORMALIZATION OF THE AGENTSPEAK EXTENSION TO DEVELOP EMPATHIC AGENTS}

$$
\begin{aligned}
& \text { EmphRegulation }(\operatorname{tr}, \omega, O, \sigma, E e)= \\
&\left\{\left\langle p^{\prime}, a^{\prime}\right\rangle \mid\left\langle p^{\prime}, a^{\prime}\right\rangle=\varphi_{2}(t r, \omega, O, \sigma,\langle p, a\rangle) \forall\langle p, a\rangle \in E e\right\}
\end{aligned}
$$

where $\operatorname{tr}$ are the personality traits of the agent, $\omega$ is the personality/emotion correlation matrix, $O$ represents the knowledge that the empathic agent knows about the target agent, $\sigma$ is the agent's current mood, and $E e$ is the set of empathic emotions. Similarly to $\varphi_{1}$ in Equation 5.13, $\varphi_{2}$ is a function that modifies the values of Pleasure and Arousal of the empathic emotion, which are represented by the tuple $\langle p, a\rangle$, using the personality, the affective link between the agents $\left(O_{a l}\right)$, and the mood $(\sigma)$.

The transition rule for the empathic regulation is defined as:

\section{$\left(\right.$ EmphReg $\left._{1}\right)$}

$$
\begin{gathered}
\frac{\text { true }}{\langle a g, C, M, T, s, M e m, T a, O, \text { EmphReg }\rangle \rightarrow\left\langle a g, C, M, T, s, M e m, T a^{\prime}, O^{\prime}, \text { EmSel }\right\rangle} \\
\text { where: } \\
T a_{E e}^{\prime}=\text { EmphRegulation }\left(T_{\varepsilon}, a g_{P_{t r}}, O, T a_{\sigma}, T a_{E e}\right) \\
T_{\varepsilon}=\langle t e, i\rangle \\
i v=\operatorname{getInteractionValue}(t e) \\
O_{a l}^{\prime}=\text { updateAl }\left(O_{a l}, i v\right)
\end{gathered}
$$

Following the previous example, let us suppose that Lily has a low positive affective link with Barney (e.g., 0.3). Let us recall that Lily's personality makes her prone to positive emotions. In addition, when Lily perceives the event, she has a positive mood (e.g., happiness). The empathic emotion regulation process will reduce the intensity of the empathic emotion and the result will be the emotion sorry for with weak intensity.

Once the regulation of the possible empathic emotions has been performed, the following step is the emotion selection (EmSel) step. 


\section{CHAPTER 5. A FORMAL EXTENSION FOR MODELLING} EMPATHIC AGENTS

\section{Emotion selection}

In the emotion selection step of the affective cycle, the final emotion $F e$ that the agent is going to simulate is selected by the function $\operatorname{SelEmotion}(\varepsilon, A e, E e, P)$ . This function has as parameters an event $\varepsilon$, the list of appraised emotions $A e$, the list of empathic emotions $E e$, and the agent personality $P$. The user can adapt this SelEmotion function to different environments and situations considering the agent personality, intensity or probability of the emotions, and other affective characteristics.

The transition rule for the emotion selection step is defined as follows:

$\left(\mathrm{EmSel}_{1}\right)$

True

$\overline{\langle a g, C, M, T, s, M e m, T a, O, \text { EmSel }\rangle \rightarrow\left\langle a g, C, M, T, s, M e m^{\prime}, T a^{\prime}, O, \text { AffAd }\right\rangle}$

$$
\text { where: } \begin{aligned}
& T a_{F e}^{\prime}=\operatorname{SelEmotion}\left(T a_{A e}, T a_{E e}, T a_{\sigma}, a g_{P}\right) \\
& a e=\left\langle T_{\varepsilon}, T a_{F e}^{\prime}\right\rangle \\
& M e m^{\prime}=M e m \cup a e
\end{aligned}
$$

This step calculates the final emotion $T a_{F e}^{\prime}$. Therefore, the affective memory Mem can be updated to store the event $T_{\varepsilon}$ with the emotion $T a_{F e}^{\prime}$ resulting from this event appraisal. This mechanism allows the agent to maintain a memory of past events and the emotion that each event produced, supporting the maintaining of long-term interactions between agents.

For instance, when Lily evaluates the event of Example 3, the self-appraisal process produces two emotions as a result: sadness with high intensity, and fear with medium intensity. Let us suppose that after the emotion regulation process the intensity of those emotions is not modified. If the user has defined the SelEmotion function to select the emotion with the highest intensity, the empathic agent will elicit the sadness emotion.

The next process of the empathic affective cycle is the affect adaptation (AffAd). 


\subsection{FORMALIZATION OF THE AGENTSPEAK EXTENSION TO DEVELOP EMPATHIC AGENTS}

\section{Affect adaptation}

In the AffectAdaptation step, the mood of the agent is updated by the function AffectAdaptation, which is defined as follows:

$$
\begin{aligned}
& \text { AffectAdaptation }(\operatorname{tr}, \omega, \sigma, F e)= \\
& \qquad\left\langle p^{\prime}, a^{\prime}\right\rangle \mid\left\langle p^{\prime}, a^{\prime}\right\rangle=\varphi_{3}(t r, \omega, F e,\langle p, a\rangle) \text { and }\langle p, a\rangle \in \sigma
\end{aligned}
$$

where $F e$ is the final emotion selected by the emotion selection process. $\varphi_{3}$ is a function that, similarly to $\varphi_{1}$ and $\varphi_{2}$ in Equations 5.13 and 5.16, modifies the mood vector, which is represented by the tuple $\langle p, a\rangle$ to adjust it to the emotion produced by the event.

\section{$\left(\operatorname{AffAd}_{1}\right)$}

\section{True}

$\overline{\langle a g, C, M, T, s, M e m, T a, O, \operatorname{AffAd}\rangle \rightarrow\left\langle a g, C, M, T, s, M e m, T a^{\prime}, P, O, \text { SelCs }\right\rangle}$

$$
\text { where: } T a_{\sigma}^{\prime}=\text { affectAdaptation }\left(a g_{P}, T a_{\sigma}, T a_{F e}\right)
$$

Since emotions and mood are expressed as vectors in the same representation space, the selected emotion will "attract" the mood to a greater or lesser extent depending on the agent's personality. As a result, the mood of the agent will be modified. Continuing with the previous example in which the selected emotion was sadness with high intensity, let us assume that Lily's mood is happiness with a low intensity. The emotion will attract the mood, and, as a result, the new mood will be sadness with a low intensity.

Once the mood of the agent is updated, the next state of the affective cycle will be the select coping strategy process. In this process, a coping strategy from the current set of applicable coping strategies will be used to create a plan that finally allows the agent to express its empathy: in its voice tone, in its speech, in its face, ... 


\section{Update time without events}

This step checks how long it has been since the agent has not received an event. If the time exceeds a certain threshold, a simulated event 'Twe event' will be triggered to elicit emotions such as boredom or sleepiness. The time without events Twe will be updated by the function updateTwe().

$\left(\mathrm{UpTwe}_{1}\right)$

Twe $>$ threshold

$\overline{\langle a g, C, M, T, s, M e m, T a, O, \text { UpTwe }\rangle \rightarrow\left\langle a g, C, M, T^{\prime}, s, M e m, T a^{\prime}, O, \text { EvClass }\right\rangle}$

$$
\begin{aligned}
\text { where: } & T_{\varepsilon}^{\prime}=\langle t, i\rangle \\
& t=\text { 'Twe_event' } \\
& T a_{A v}^{\prime}=\left\langle a v_{1}, a v_{2}, \cdots, a v_{n}, T w e\right\rangle \\
& T w e=\text { updateTwe }()
\end{aligned}
$$

$\left(\mathrm{UpTwe}_{2}\right)$

$$
\begin{gathered}
\frac{\neg \text { Twe }>\text { threshold }}{\langle a g, C, M, T, s, \text { Mem, Ta, O, UpTwe }\rangle \rightarrow\left\langle a g, C, M, T, s, M e m, T a^{\prime}, O, \text { UpTwe }\right\rangle} \\
\text { where: } T a_{A v}^{\prime}=\left\langle a v_{1}, a v_{2}, \cdots, a v_{n}, \text { Twe }\right\rangle \\
\text { Twe }=\text { updateTwe }()
\end{gathered}
$$

\subsection{Conclusions}

Empathy is a key element in social interactions affecting human affective states and behaviours and provides a basis for long-term relationships. Several proposals have been made from the area of affective computing to simulate empathic behaviors. These proposals are generally based on theoretical models from psychology, sociology, and neuroscience. Most computational 


\subsection{CONCLUSIONS}

models for simulating empathic behaviors are generally based on the agentoriented programming paradigm. However, these proposals are designed ad hoc, generating agent programs that are highly dependent on the programmer and that are difficult to understand, share, and re-use. In this paper, we have presented a model of empathic agent that is based on the BDI language AgentSpeak. Our proposal is based on the theories of empathic appraisal and emotion regulation. We have extended the syntax, semantics, and the reasoning cycle of the AgentSpeak language to support the design of empathic agents. We have modified the affective cycle that had been incorporated to AgentSpeak by GenIA ${ }^{3}$. This has added new processes and functionalities to allow the elicitation of empathic emotions. We have formalized our extension using the same operational semantics formalism used by AgentSpeak and $G e n I A^{3}$. This operational semantics defines the structure and the configuration of the agent. The formalization of all of the transitions guarantees the validity of our proposal.

Our empathic model is structured around three fundamental processes: perception, empathic appraisal, and empathic regulation. After perceiving an empathic event, the empathic appraisal process elicits a set of empathic emotions. These empathic emotions are then adapted by the empathic regulation process according to the knowledge that the agent has about the target agent.

The new affective cycle is divided into two different appraisal processes: one for eliciting empathic emotions induced by the perception of an emotion or situation in a target agent; and another for eliciting emotions based on self-directed or environmental events. This division of the affective process allows the agent to be able to maintain a sense of self that is distinct from the target agent.

The use of the affective link representing the affective proximity between two agents allows the personalization of the empathic response based on the target agent. In addition, a correlation matrix represents how each personality trait of the agent influences the emotion that will be elicited. Finally, in our proposal, agents maintain a memory of past events and the emotion that the past events produced in the agent. This affective memory, combined with the possibility of establishing and modifying the affective links with other agents, 


\section{CHAPTER 5. A FORMAL EXTENSION FOR MODELLING}

EMPATHIC AGENTS

allows the simulation of personalized long-term interactions, which are crucial in affective interactions in human beings. All of these characteristics of our empathic agent model will produce software agents that are more realistic when modelling human organizations.

We are currently considering increasing the agent's knowledge of other agents by adding new structures to store the beliefs, goals, and concerns of other agents using state-of-the-art approaches of Theory of Mind. Moreover, the affective link can be complemented maintaining a level of trust in other agents. This will improve the perspective-taking process so that the agent can evaluate perceived situations using more knowledge about the target agent. 



\title{
Chapter 6
}

\section{Towards a model of empathic pedagogical agent for educating children and teenagers on good practices in the use of social networks}

\author{
Joaquin Taverner, Emilio Vivancos, and Vicente Botti
}

Int. Conf. on Agents and Artificial Intelligence (ICAART), pages 439-445, 2021

Doi: https://doi.org/10.5220/0010345504390445

\begin{abstract}
Social networks have been a revolution for our society. The rapid expansion of these networks results in more and more children and teenagers using them regularly. However, despite the fact that most social networks have privacy control systems, most young people do not use these controls because teenagers are usually unaware of the privacy risks that exist in the Internet. This inadequate use of social media can lead to different social problems such as cyber-bullying, grooming, or sexting. The best way to prevent these risks is through education. In this paper we propose an empathic pedagogical agent model for education on good practices in the use of social networks. The agent interacts empathetically with users through the social network. The user's emotion is recognized through a camera and is processed in real time to obtain the emotion. The agent analyzes the recognized emotion and the users' actions and looks for the best strategy to advice and educate the teenager in the correct use of the social network.
\end{abstract}




\subsection{INTRODUCTION}

\subsection{Introduction}

Today's society is increasingly moving towards virtual environments. Video calls, instant messaging, and social networks have transformed the way we communicate and relate to others. The incorporation of these new technologies into our daily lives, has been accelerated this year by the pandemic produced by COVID-19. People find several advantages such as instant communication, the search for lost old friends, the improvement of the prospects of finding a job, or the communication in times of confinement. The democratisation of the use of the Internet is resulting in more and more children and teenagers using them. In fact, today the young people are the biggest consumers of technology and social networks [79]. However, young people are often not aware of the privacy risk they are exposed to when using the Web [3]. An inappropriate use of social networks can have negative personal, professional, and emotional consequences [144]. These consequences are aggravated when they occur in children and teenagers, as they do not have sufficient emotional capacity to deal with certain situations that may arise from the inappropriate use of social networks. Therefore, for a social transition towards virtualised environments to be effective, it is necessary to design educational models focused on teaching the correct use of the Internet. An appropriate use of the web can turn it into a pedagogical tool that allows a great dissemination of knowledge [114]. Artificial intelligence can help in this educational task through the use of intelligent agents acting as tutors in the use of social networks. Intelligent agents have already been used in education with good results [159, 207]. In particular, agents with empathic abilities, capable of recognising and showing emotions, have been successfully used in educational tasks by increasing the sociability and participation of young people [188]. Furthermore, empathic intelligent agents are perceived as much more reliable than traditional agents by children and teenagers. This advantage makes empathic agents more suitable for tutoring [165]. In this paper we propose a model of empathic pedagogical agent to be used as a tutor to train young people in the correct use of a social network.

The rest of this paper is organized as follow. In section 6.2, we discuss the problem of privacy risk in the Internet and the most important proposals 
regarding pedagogy with agents and education in the use of social networks. Section 6.3 introduces a empathetic pedagogical agent model for education on social networks. The agent will interact with students in an affective way by recognizing and expressing emotions. Finally, the main conclusions and future works are presented in Section 6.4.

\subsection{Related work}

Social networks have revolutionised various aspects of society. They have not only transformed the way we communicate and relate to others, but have also affected other aspects such as politics, business, culture, or the way we perceive reality. Social networks also allow us to meet new people and share our ideas. However, not everything is an advantage in social networks. These networks have become a perfect medium for the dissemination of fake news and disinformation campaigns. Fake news try to give a biased view of many events in order to manipulate people's opinion.

Given the high level of social exposure to which users of social networks are subjected, there is a risk of losing privacy. In addition, an inappropriate comment or an unfortunate photo can harm our personal image and damage us socially and emotionally. In fact, the privacy risk has become one of the main concerns of users of this type of network [3]. Currently, most social networks have privacy management systems that allow users to control the audience of their publication. However, these systems are often ignored by users, both because they are not aware of the potential risks of exposure of a specific publication and because users are not concerned about privacy. This fact is further aggravated when young people are involved since they do not have sufficient social and emotional skills to deal with certain situations that may arise when using social networks [156].

Nowadays, the younger population is becoming the main users of social networks. The European General Data Protection Regulation (GDPR) [110] allows young people over 16 to transfer their personal data to be processed by social network providers without the consent or the authorization of the parental responsibility holder. In addition, this regulation allows EU mem- 


\subsection{RELATED WORK}

bers to reduce this age to 13 years. Consequently, children and teenagers spend several hours a day using their smartphones. During this time, they receive hundreds of advertisements and messages that, frequently are hoaxes and fake news. This misinformation is often spread to create a negative view of everything that is different: religious belief, political ideas, sexual tendency, immigrants, people with disabilities,... This misinterpretation and manipulation of real events can affect the emotional development of teenagers and children, because they have less critical thinking and tools to analyse and protect themselves of fake news. Moreover, during pandemic confinement, young people have spent many hours connected to the Internet, often without parental supervision [87]. How can they filter all the messages that receive? Ballesteros and Pizaco [14] estimated that only 22\% of teenagers between 14 and 16 years old have received training on how to filter the information they find on the Internet.

On the other hand, many young people use social networks as a social showcase in which they look for group acceptance: the greater the number of followers and friends, the better their perception of their status on the social network. However, most young people do not know how to handle their privacy in social network environments [210]. Therefore, when making a publication they do not consider the risk that sharing this information may suppose. This is because they do not have the necessary skills to determine what kind of information poses a risk when disseminating it. This level of public exhibition can have negative consequences. Recent studies show that the abusive and inappropriate use of social networks can lead to negative effects such as addiction, cyber-bullying, grooming, sexting, and paradoxically, social isolation $[144,178]$.

\section{Pesedia: a pedagogical social network}

In recent years, some proposals for assessing privacy on social networks have been made $[1,4,29]$. Most of these proposals involve the definition of metrics and mechanisms to improve trust and avoid the vulnerability of privacy in social networks $[191,218]$. One of these proposals is found in [9] in which Pesedia, a social network designed specifically to educate in the correct use of 
social networks, is presented. Pesedia offers an interface that is very similar to the interfaces of current social networks like Facebook. Young people have their own wall where they can create and share publications. They can also send private messages or establish groups. The network also allows to establish friendship relationships and trust levels in order to teach the appropriate privacy measures by providing the appropriate level of privacy for the publications. Pesedia allows the addition of plugins to increase its functionality. For example, in [3] two mechanisms were included to assist in the decision-making process of selecting the privacy level of a publication. The aim of these mechanisms was to alert users about the visibility of publications in order to make them aware of the privacy risk involved when posting. These mechanisms were based on the model of soft-paternalism, in which specific interventions are made in the actions performed by the student without affecting his/her freedom of choice.

\section{Pedagogical intelligent agents}

While the establishment of metrics and mechanisms has proven to be effective in educating about good practices in social networks, they lack the interactive experience that an intelligent agent can offer. Intelligent agents are capable of reasoning and reacting proactively to a perceived change in the environment. Therefore, they are able to interact with the user by reacting to his/her actions. Intelligent agents have been used in recent years to perform tutoring tasks in educational environments improving the motivation, socialization, understanding, and attitudes of students [188]. For example, an educational pedagogical agent model for tutoring children on the autism spectrum is proposed in [89]. Similarly, in [16] an educational pedagogical agent model for helping in homework is presented. That agent was developed to operate in a social network in which students perform their homework while participating in a common social experience.

Many proposals made in the field of affective computing focus on the study of empathy as a mechanism to improve the human-machine interaction [165]. Empathy is a construct used in different domains such as ethology, philosophy, psychology, or neuroscience to explain different social behaviours. These 


\subsection{RELATED WORK}

behaviours are generally focused on the ability to understand and share emotions, moods, and mental states of others, as well as the behaviours derived from this understanding, such as prosocial behaviour or altruism [49, 213]. Empathy encourages the development of emotional bonds, allowing human beings to constitute themselves as social and moral beings [119]. In fact, recent neuroscientific studies relate some social disorders, such as autism spectrum disorder or psychopathy, with a deficit in the brain areas involved in empathic processes [21]. One of the most recurrent definitions of empathy is the one proposed by M. L. Hoffman [99], in which empathy is defined as a psychological process that makes a person have feelings more similar or congruent with the other person's situation than with his/her own situation. Therefore, it is not necessary for empathic responses to be identical or approximate to the mood of the other, but according to Hoffman any emotional reaction compatible with the mood of the other can be considered empathy. According to Hoffman's definition, to simulate the processes inherent within empathy, software agents must be able to perceive and recognize emotions or moods and react accordingly by simulating a behavior appropriated to the perceived emotion or mood.

The area of artificial intelligence has evolved by developing models of agents with different affective and empathic abilities [7, 6, 216, 234]. Most of these models use a dimensional representation for emotions and mood [220]. One of the most widely used dimensional representation is the Circumplex Model of Affect that uses two dimensions to represent emotions: pleasure and arousal [193]. The use of this dimensional model allows the representation and simulation of affective processes such as emotion elicitation, emotional contagion, or empathy.

Empathic agents have been used in different contexts and have proved to be more reliable and more credible, thus reducing the stress and frustration of human users and improving human-machine interaction [165]. The development of agents with empathy has also been useful in the educational field. For example, the research performed in [188] used empathic agents to prevent bullying. In this experiment it was found that agents with empathic skills increased the sociability and involvement of young users. The participants played a game in which the agents recognized and showed emotions. 

AGENT (SELECTED PAPER)

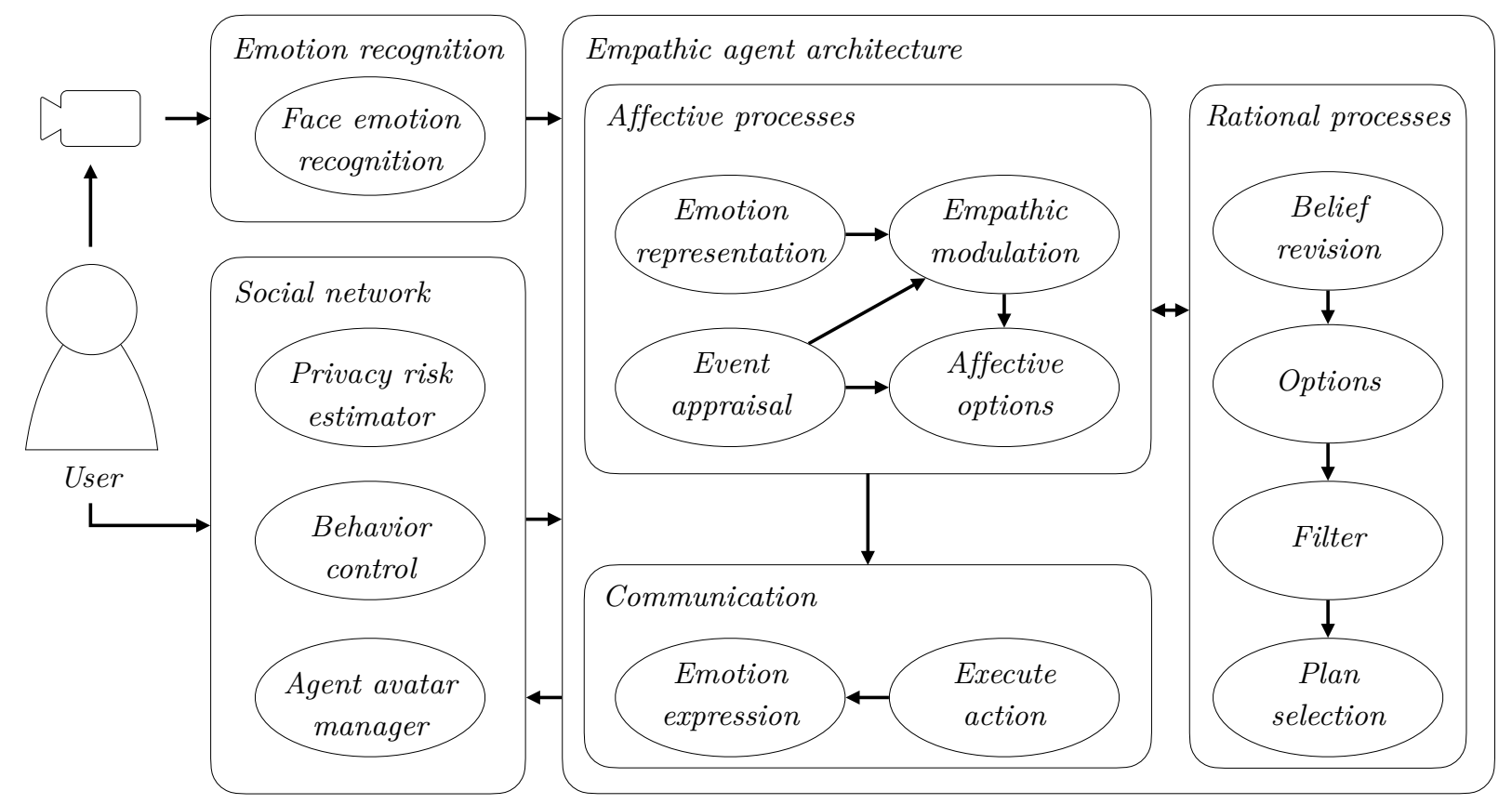

Figure 6.1: The proposed model for a empathic pedagogical agent in a social network

During the game, a series of social situations were presented in which an agent was bullied by other agents with the intention of driving the students' behaviour through empathy. Empathic agents have also been used for tutoring in educational environments. For example, in [159] a robot tutor with empathic skills was proposed. The study showed that the empathic agent improved the predisposition of young people to participate in learning experiences. In the same way, in [207] a model of a robot tutor with empathy is presented. This model is able to recognize children's emotions and interact with them through a touch screen. The results of experiments show that the empathic robot tutor was able to elicit and maintain the social engagement of the students.

Considering the promising results of previous works, where was shown that empathy increases trust and engagement, we propose to employ an empathic agent to increase the efficiency of the learning process of young people in the correct use of social networks. 


\subsection{PROPOSAL}

\subsection{Proposal}

As mentioned in the previous sections, irresponsible use of social networks can compromise the user's privacy. Therefore, it is necessary to develop tools that allow young users to understand the risks to which they are exposed and to learn good practices in the use of social networks with the aim of maintaining their privacy. In this paper we present an educational pedagogical agent model with empathetic abilities. Our agent interacts with the user through the social network. The agent will monitor the user's actions on the network to analyse his/her behaviour. When the user prepares a publication, the agent analyses the privacy risk associated with the publication and recommends the best strategy for avoiding privacy risks. Following a soft-paternalism strategy, the user will be free to decide how to carry out the publication. Once the publication has been made, the agent will react according to whether the user has followed its advice or not.

In order to improve the interaction with the user, the agent must have certain social and emotional capacities to react with an appropriate behaviour. One of the best approaches to generating this social behaviour is through agents that simulate empathy. Our proposed agent uses empathy as a tool to build trust with the user improving its educational task. Through empathy, our agent is able to react appropriately according to the emotion or mood of its young interlocutor. Following the definition of empathy proposed by Hoffman, our agent will be able to recognize the emotion in the teenager and to produce a behavior according to that emotion. The model that we propose in this paper uses a real-time facial emotion recognition system. Once the emotion is recognised, the agent represents the emotion internally using a multidimensional representation model [220] and generates an empathic response in accordance with the perceived emotion. In addition, the agent will have a proactive emotional behaviour depending on the actions performed by the user in the social network.

Figure 6.1 shows the model for the interaction between the young user and the agent in the social network. The framework proposed in this work consists of three main components: the social network based on Pesedia, the emotion recognition system, and the empathic intelligent agent. 


\section{Pesedia}

Our proposed framework uses three plugins of the Pesedia social network: the privacy risk estimator, the behavior control, and the agent avatar manager. On the one hand, the privacy risk estimator is responsible for estimating the potential audience for a publication. This estimation is obtained by measuring the number of potential users that can access to the publication according to the degree of privacy that the user has selected [2]. On the other hand, the behavior control is used to capture the interactions that the user makes within the social network, for example, when pushing a "like" button or when posting an item. Finally, the agent avatar manager displays the agent's facial expressions and messages on the social network window. Figure 6.2 shows a screenshot of Pesedia with the agent avatar interface on the right. When the young user makes a comment, the privacy risk estimator warns the user of the risk involved in such publication. In the example in Figure 6.2, the user has provided a location that could lead to a high privacy risk. The agent warns the user that the publication has a high risk and recommends to modify the audience. The final decision belongs to the user: the teenager can continue with the publication or modify the audience. Depending on his/her decision, the agent will adapt its behaviour by using different messages and emotional expressions.

\section{The emotion recognition system}

The emotion recognition system consists of a video camera and a classifier based on convolutional neural networks that is locally installed in the device used by the teenager. This system captures the image in real-time and through a cascade classifier [209] obtains the position of the user's face. Then the face image is sent to a convolutional neural network installed in the computer used by the user. This convolutional neural network obtains the most likely emotion according to the user's facial expression [74]. To avoid microexpressions the system performs a controlled analysis using a multiple frame analysis. It is important to remark that the empathic agent can only be as good as the accuracy of the recognised emotion. 


\subsection{PROPOSAL}

\section{Empathic intelligent agent}

Experts recommend improving the critical thinking of young people. This process involves detecting and analysing all the messages and information that the teenager receives, but frequently young people navigate the Internet without the supervision of any adult. In this kind of scenarios, an intelligent agent supervising all the teenager's activity on the Internet can provide the advice and emotional support that absent adults cannot give. Our proposal of architecture for this intelligent agent is composed of three modules: the rational processes module, the affective processes module, and the communication module.

Intelligent agents must be able to reason and establish plans. In our proposal the rational processes module is based on the BDI architecture model. A BDI agent is composed of a set of beliefs, desires, and intentions. The beliefs represent the information that the agent has about the state of the environment in which it is located, in this case the social network. The desires are objectives that the agent wants to achieve. In our model, the objectives are a set of pedagogical actions such as persuading the user to change the privacy of a message. Finally, the intentions are the objectives that the agent selects to execute them.

The affective processes module is composed of four processes: the appraisal process, the emotion representation process, the empathic modulation process, and the selection of affective options process. The appraisal process appraises any action of the young users and determines the emotion. Appraisal theories hold that when a stimulus is procured, a set of processes are triggered to evaluate that stimulus [221]. As a result of these processes an emotion is generated. In our model, when the agent perceives an action of the user in the social network, the agent evaluates this action according to its beliefs and its desires and, as result, an emotion is triggered. This emotion is represented in a two dimensional space based on the dimensions of pleasure and arousal by the emotion representation process. Then, the empathic modulation process uses the dimensional representation to approximate the emotion elicited by the appraisal process to the emotion recognized in the user's face. As a result of this process, an empathic emotion that will be more in line with the user's 

AGENT (SELECTED PAPER)

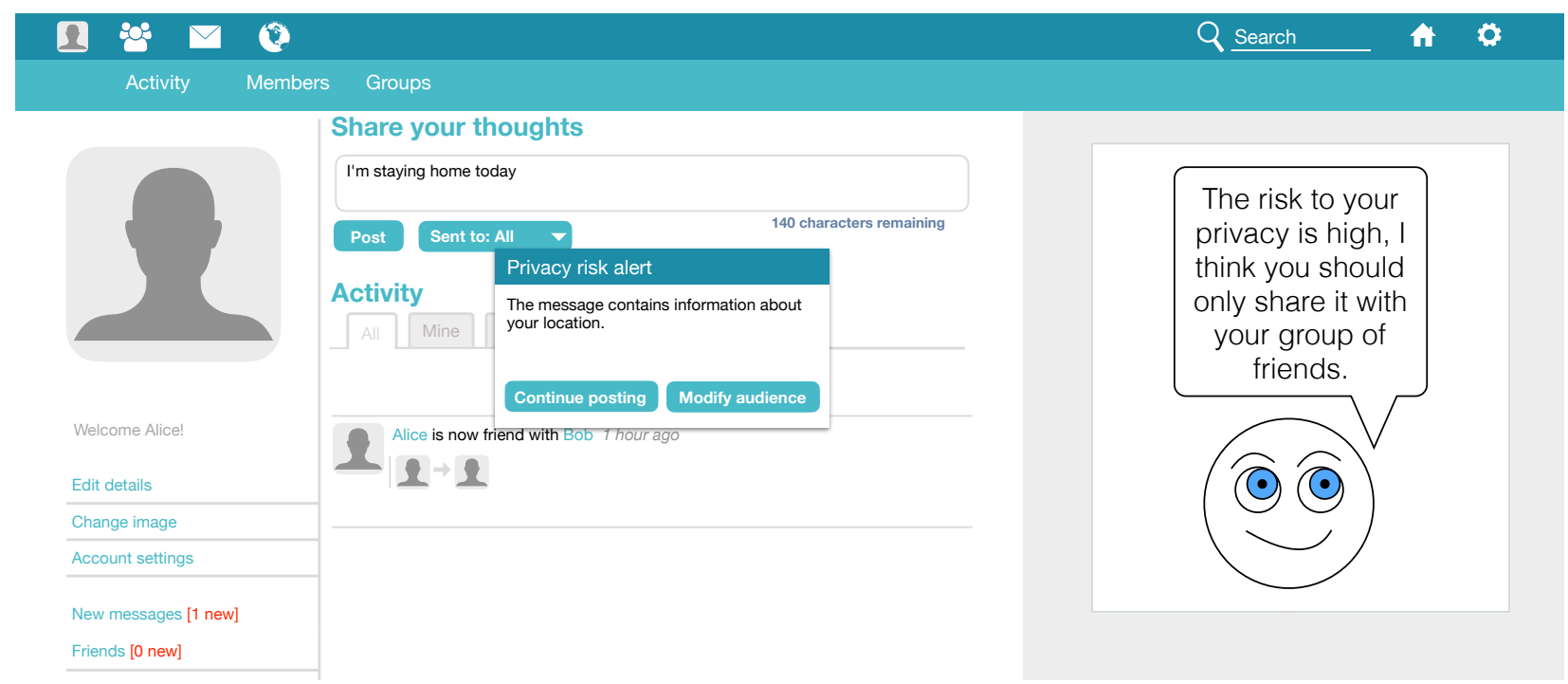

Figure 6.2: Screenshot of the Pesedia social network with the agent avatar interface on the right side.

emotional state is generated. Finally, this empathic emotion is used by the selection of affective options process to identify the applicable plans.

The last component is the communication module. This component is responsible for transmitting the actions that must be performed to the agent event manager. It is composed of two processes: the execute action process and the emotion expression process. The first one is in charge of executing the plans that have been selected by the plan selection process from the plans generated in the affective options and the filter processes. These plans may contain educational actions, privacy recommendations, or affective expressions. Finally, the emotion-expression process selects the facial expression that the agent must show, and sends it to the agent avatar manager (see Fig 6.2).

\subsection{Conclusions and future work}

Today, social networks are an essential part of many young people's lives. However, using social networks without the necessary skills represents a privacy risk. The best tool to fight the privacy risk is education. In this work we 
have proposed a model of a pedagogical empathic agent for education in the use of social networks. The agent interacts with young users warning them about privacy when a publication is made. The agent interacts with the user through the social network. To make this interaction as natural as possible we have proposed the use of empathy that has been used before in teaching tasks obtaining good results.

Our empathic pedagogical agent model will be tested on Pesedia social network to determine if it improves the perception of the risk of privacy of young people. Further experiments will be performed to test whether the agent is able to provide young people with the necessary skills to manage privacy risk in social networks. 


\section{Part III}

\section{Discussion}





\section{Chapter 7}

\section{Discussion}

Empathy is a critical component of social interactions that affects affective states and human behaviors and provides a foundation for long-term relationships. From a computational perspective, the development of systems capable of simulating abilities related to social cognition, such as empathy or Theory of Mind, requires the design of processes to identify or perceive the mental state of other agents in order to provide an appropriate empathic response. For this response of the agent to be as human-like as possible, it has to be adapted, through a process of regulation, to different affective, intrapersonal and interpersonal factors. To adapt empathic responses to these factors, it is necessary to use models for the representation of affective states that make it possible to represent, in a common space, the affective states of the agent and the affective states identified or perceived in the other agents. In this sense, theories such as the Core Affect theory, make it possible to represent affective states in a dimensional space and to establish relationships between the different affective states. However, as discussed in Section 2.2, proposals made to date in affective computing that used dimensional models present several disadvantages that make it difficult to extrapolate them to an empathic agent model. Therefore, this work proposes a new representation model based on a polar coordinate system composed of the pleasure and arousal dimensions, in which the affective states are represented by vectors. This model defines different types of intensity for affective states as a function of the vector module.

Furthermore, for an empathic agent to interact with human users a rep- 
resentation model capable of representing and expressing emotions using the same words and expressions that humans use is needed. However, it must be considered that these words are language and culture dependent, that is, the literal translation of the terms from one language to another can produce biases or misinterpretations in the representation model. Therefore, in this thesis a new methodology based on experiments to adapt the emotion representation according to the culture and language in which it will be used is designed. This methodology is based on two experiments: the first experiment is used to study the most appropriate translations of the dimensions of pleasure and arousal in a target language; and the second experiment is used to determine the distribution of emotions along these two dimensions. The results of the first experiment with 50 Spanish participants helped to corroborate the importance of adapting the translation of emotional models by considering the different meanings that humans give to emotion labels in different cultural environments. For example, the affective category "Misery", used in the literature as the antagonistic value of Pleasure and whose closest translation into Spanish is "Misera", was not selected by the participants of the first experiment as representative of this affective category. This may be due to the fact that, in Spanish, the concept of "Miseria" is more associated with poverty than with displeasure or suffering. On the other hand, the results of the second experiment, conducted with 100 participants, helped to determine the levels of pleasure and arousal associated with the different emotions in Spanish. These experiments were performed with Portuguese and Swedish participants to adapt the model to those cultures and languages. In this case, the results allowed to highlight the differences between the three languages. For example, for some emotions, Spanish and Portuguese had a greater similarity than when compared to Swedish. These results are in line with the results obtained in previous experiments in which similarities in the conceptualization of emotions between language families were already reported. Another interesting result provided by these experiments was that there were no significant differences between the association of the dimensions of pleasure and arousal for each emotion according to gender or age.

From the results obtained in the experiments, a probabilistic model in which different probability regions are defined for each emotion was gener- 
ated. In this way, the direction of the vector composed by the pleasure and arousal dimensions indicates the probability of membership of that vector to a certain affective state. This model combines probability regions with different levels of intensity, defining each affective state by an area instead of a single point. The probability areas can be easily modified to adapt the model to the cultural environment in which it is being used. In fact, through this multidimensional model of affect, an agent can have different probability models for different languages and adapt its model of representation and expression of affective states to the language and culture of its human interlocutor. To do that, two processes based on the different cultural models are established: a defuzzification process, which allows to represent words related to affective states in the space of pleasure and arousal adapted to the cultural environment; and a fuzzification process, which determines an emotion label in a specific language from a pair of pleasure and arousal values. These processes allow the agent to express its affective state and to represent the perceived affective state using the same interpretation of the fuzzy emotional terms used by its human interlocutor.

Another fundamental aspect in the development of agent models capable of simulating affective behaviors, is the way in which emotions are elicited in the agent. Based on appraisal theories, which indicate that emotions are the result of an appraisal of a perceived stimulus, a new computational appraisal model that can elicit more than one emotion in each appraisal cycle is established. This model is capable of eliciting ten different types of emotions with different intensities with a small number of fuzzy rules. To do that, this model uses five fuzzy appraisal variables to determine the candidate emotions to be elicited when perceiving an event through the Event appraisal process. These emotions are expressed, using vectors, in the multidimensional culturally adapted representation model. Then, through an affect adaptation process, the agent's mood is adapted using these emotions together with the agent's personality. As a result, different agents, with different personalities, show a different mood when they perceive the same event. To establish the influence of personality, a correlation matrix is defined. This correlation matrix can be adapted to different theories and approaches and represents how each agent's personality trait influences the emotion that will be elicited. 
Once it is established how affect is represented, how affect is communicated between agents and humans, and how emotions are elicited, a formal model of an affective agent with empathic abilities is defined. This model departs from the proposals made in the field of affective computing, which generally focused on the definition of ad-hoc models. Using the generic architecture for affective BDI agents $G e n I A^{3}$, which uses the well-known agent-oriented language AgentSpeak, the empathic agent model offers low programmer dependency. To this end, the syntax, semantics, and the reasoning cycle of AgentSpeak are extended to support the development of agents that are capable of performing empathic interactions. A new inference model is also proposed to allow emotions and empathy to have an effect on the practical reasoning of the agent. Emotions and empathy will be involved in both the deliberation process when the agent is selecting an intention to commit, and in the means-end reasoning process when the agent is deciding which plan to follow. To facilitate the emergence of empathy in software agents, the GenIA ${ }^{3}$ architecture is extended with a new syntax and semantics.

In order to provide an agent with the ability to perform an empathic reasoning process based on perspective-tacking, it is necessary to clearly identify the participants involved in an empathic interaction. In the proposed model, four types of agents involved in an interaction are identified: the agent that performs the action, which is labeled as the subject agent; the agent that receives the consequences of the action, which is labeled as the target agent; and the agent that perceives the action, which is labeled as the observer agent. The syntax of an AgentSpeak event to incorporate the knowledge about the participants of the event is also redefined. In this way, an agent is able to reason about events that have happened to other agents and, therefore, to react empathically to these events through a process of perspective taking.

To allow the emergence of empathic interactions, the practical and affective reasoning processes of an agent are modified. In addition, three new processes for eliciting empathic emotions based on theories of empathic appraisal and emotion regulation are designed: percepción, empathic appraisal, and empathic regulation. After perceiving an empathic event by the percepción process, the empathic appraisal process elicits a set of empathic emotions using a self-projection approach (in which the agent evaluates the event perceived 
in another agent as if the event had happened to him/her) and considering different appraisal variables. These empathic emotions are adapted by the empathic regulation process according to the knowledge that the agent has about the target agent. For this purpose, the affective link is used. The affective link represents the affective proximity between two agents and allows the personalization of the empathic response according to the target agent. Affective link is combined with affective memory (which stores past events together with the emotion that those events produced in the agent) for the simulation of long-term personalized interactions, which are critical in affective interactions in humans. Moreover, two types of events are distinguished: affective events and empathic affective events. Each type of event is evaluated by a different appraisal process providing the agent with the cognitive ability to distinguish the self from the others that is, according to the literature, a fundamental component in the development of cognitive empathic reasoning. All these features of this empathic agent model will lead to more realistic software agents when modeling human behavior, human organizations, and human-machine interactions.

Finally, this empathic agent model has been adapted for its use in a social network with the objective of teaching young people about the appropriate and secure use of social networks. The agent interacts with teenagers by warning them about privacy and providing guidance when posting. To make the interaction natural and believable, the agent recognizes the user's emotion and represents it using the proposed representation model adapted to its cultural environment. This emotion is used to estimate the empathic response of the agent and to adapt its behavior to the affective state of the user. 


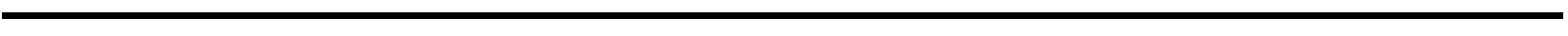




\section{Part IV}

\section{Conclusion and future work}





\section{Chapter 8}

\section{Conclusion and future work}

Empathy is a fundamental component in the understanding of human social and affective behavior. However, there is still much work to be done from different areas such as psychology, sociology, or neuroscience to understand the immensity of the cognitive processes that allow the emergence of affective and social phenomena such as empathy. There are currently several theories that attempt to explain the phenomenon of empathy. The trend seems to be in line with the development of theories focused on empathic appraisal. Empathic appraisal theories suggest that empathy is generated as a result of an appraisal process together with a regulation process that allows adapting the empathic response to different social and affective factors.

The fields of computing that study the simulation of human behavior, the behavior of human social organizations, and human-machine interaction may benefit from the introduction of concepts from empathy-centered theories. Computational systems capable of simulating different aspects related to empathy contribute to improve the understanding of the factors that produce this phenomenon as well as to understand the bonds that arise in human societies. Experiments with agents capable of simulating different levels of empathy have shown that human users perceive these agents as more human-like, credible, and trustworthy. Therefore, the simulation of empathic emotional behavior allows to improve the user experience and to advance in the development of models that improve the paradigm of human-machine interaction.

However, to design this type of systems it is necessary to establish formal models that define the processes that allow the development of empathy 
simulation and common representation systems of affective states for both software agents and humans. Furthermore, given the way in which humans express their emotions through labels or words, it is necessary to develop computational models capable of reasoning and simulating affective behavior by understanding and expressing affective states in the same terms as humans. However, it is necessary to consider that these terms are dependent on the cultural environment, that is, different cultures may have different ways to express the same affective state. Therefore, translating the representation models literally can lead to errors or misinterpretations affecting the simulation and the user's experience.

The work performed in this thesis represents an advance in the field of affective computing with the contribution of a model for the representation of affect and the simulation of affective and empathic behavior in multi-agent systems. The proposed model of affect representation allows, as an innovation, to represent emotions using areas in the space of pleasure and arousal instead of simple points. A emotion is represented using a vector in a polar coordinate system in which the direction of the vector indicates the type of emotion and the module of the vector determines its intensity. Through this representation it is possible to simulate different behaviors related to affective states such as the intensity of emotions or mood mitigation. In addition, having a common multidimensional representation space allows to explore affective states and adapt them to different factors (such as personality) to provide personalized responses and more realistic behaviors.

In this work, a new experiment-based methodology that allows to adapt the multidimensional model of affect representation to different cultural environments is presented. Thereby, the same agent can have different probabilistic models to adapt the emotion and express it using the same terms and in the same language of his human interlocutor, reproducing a more natural and credible simulation and avoiding possible misunderstandings or misinterpretations resulting from the different languages.

To provide agents with the ability to simulate an affective reasoning process as human-like as possible, a new computational appraisal model is defined. This model uses fuzzy logic and allows the definition of different levels of intensity for emotions. Through a fuzzification and a defuzzification processes, 
the agent is able to recognize and express the affective states using the same terms and in the same language as its human interlocutor. Furthermore, using the multidimensional model of affective state representation, a formalization for mood adaptation is proposed as a function of the emotions generated by the fuzzy appraisal and the agent's personality. In this way, different agents, with different personalities, can exhibit different behaviors when perceiving the same event.

All these advances have been used to define a new agent model capable of simulating empathic abilities. Using the well-known AgentSpeak agent language, several processes have been identified and defined to simulate the cognitive processes of affective appraisal, empathic appraisal, and empathic regulation (among others). These processes allow agents to select the appropriate affective or empathic response when perceiving an event. The processes of affective appraisal and empathic appraisal also allow the agent to simulate the distinction between the self and the other, which is identified in the literature as a fundamental component in the development of the cognitive concept of empathy and Theory of Mind.

Therefore, this thesis represents a significant contribution to the computational representation of information related to affective states as well as to the modeling of agents with empathic and Theory of Mind abilities. However, much work remains to be done in the field of affective computing to achieve a realistic simulation of human affective behavior. Thus, some future challenges are identified. On the one hand, the representation model considers areas in a two-dimensional space formed by the pleasure and arousal components. It would be interesting to study the effect of adding more dimensions to the representation model, such as Dominance, as these additional dimensions may be useful to disambiguate the possible overlaps that occur between some emotions in the two-dimensional model. It would also be interesting to analyze the influence of these dimensions on social behavior by simulating it through a multi-agent system, as well as to study the influence of these dimensions across cultures. On the other hand, regarding the simulation of empathic behavior, it would be interesting to further enhance the agent's ability to use information about other agents by adding new structures to store the beliefs, goals, and concerns of other agents using state-of-the-art Theory of Mind ap- 
proaches. In addition, the affective link representation can be enhanced by maintaining a level of trust in other agents. This will improve the perspectivetaking process so that the agent can evaluate perceived situations using more knowledge about the target agent. Finally, it is necessary to develop new experiments to test whether the proposal of an empathic pedagogical agent is able to provide young people with the necessary skills to manage privacy risk in social networks. 


\section{Bibliography}

[1] Alessandro Acquisti, Idris Adjerid, Rebecca Balebako, Laura Brandimarte, Lorrie Faith Cranor, Saranga Komanduri, Pedro Giovanni Leon, Norman Sadeh, Florian Schaub, Manya Sleeper, et al. Nudges for privacy and security: Understanding and assisting users' choices online. ACM Computing Surveys (CSUR), 50(3):1-41, 2017.

[2] José Alemany, E del Val, J Alberola, and Ana García-Fornes. Estimation of privacy risk through centrality metrics. Future Generation Computer Systems, 82:63-76, 2018.

[3] José Alemany, Elena del Val, J Alberola, and Ana García-Fornes. Enhancing the privacy risk awareness of teenagers in online social networks through soft-paternalism mechanisms. International Journal of HumanComputer Studies, 129:27-40, 2019.

[4] José Alemany, Elena del Val, and Ana García-Fornes. Assisting users on the privacy decision-making process in an osn for educational purposes. In International Conference on Practical Applications of Agents and Multi-Agent Systems, pages 379-383. Springer, 2020.

[5] Bexy Alfonso, Emilio Vivancos, and Vicente Botti. An open arquitecture for affective traits in a BDI agent. Proc. of the 6th ECTA 2014. Part of the 6th IJCCI 2014, pages 320-325, 2014.

[6] Bexy Alfonso, Emilio Vivancos, and Vicente Botti. Toward formal modeling of affective agents in a BDI architecture. ACM Trans. on Internet Technology, 17(1):5, 2017. 
[7] Bexy Alfonso, Emilio Vivancos, Vicente Botti, and Penélope Hernández. Building emotional agents for strategic decision making. In Proc. of the Int. Conf. on Agents and Artificial Intelligence, volume 2, pages 390397. SCITEPRESS, 2015.

[8] Selim Aren and Hatice Nayman Hamamci. Relationship between risk aversion, risky investment intention, investment choices: Impact of personality traits and emotion. Kybernetes, 2020.

[9] Estefanía Argente, Emilio Vivancos, José Alemany, and Ana GarcíaFornes. Educating in privacy in the use of social networks. Education in the Knowledge Society, 18(2):107-126, 2017.

[10] Paul J Argott, Dawn Buffington Townsend, and Claire L Poulson. Acquisition and generalization of complex empathetic responses among children with autism. Behavior analysis in practice, 10(2):107-117, 2017.

[11] Aladdin Ayesh and William Blewitt. Models for computational emotions from psychological theories using type-ii fuzzy logic. Cognitive Computation, 7(3):309-332, 2015.

[12] Bab Dictionary. Bab English-Swedish Dictionary. https://sv.bab.la, 2021 (accessed September 17, 2021).

[13] Kaveh Bakhtiyari and Hafizah Husain. Fuzzy model of dominance emotions in affective computing. Neural Computing and Applications, 25(6):1467-1477, 2014.

[14] Juan Carlos Ballesteros and Laura Picazo. ICTs and their influence on adolescent socialization. (in Spanish). Madrid: Centro Reina Sofia sobre Adolescencia y Juventud Fundacion de Ayuda a la Drogadicción, 2018.

[15] Lisa Feldman Barrett, James Gross, Tamlin Conner Christensen, and Michael Benvenuto. Knowing what you're feeling and knowing what to 
do about it: Mapping the relation between emotion differentiation and emotion regulation. Cognition \& Emotion, 15(6):713-724, 2001.

[16] María Lucía Barrón-Estrada, Ramón Zatarain-Cabada, Yasmín Hernández Pérez, et al. An intelligent and affective tutoring system within a social network for learning mathematics. In Ibero-American Conference on Artificial Intelligence, pages 651-661. Springer, 2012.

[17] Erdenebileg Batbaatar, Meijing Li, and Keun Ho Ryu. Semanticemotion neural network for emotion recognition from text. IEEE Access, 7:111866-111878, 2019.

[18] Edward Batschelet. Circular statistics in biology. ACADEMIC PRESS, 111 FIFTH AVE., NEW YORK, NY 10003, 1981, 388, 1981.

[19] Christopher Beedie, Peter Terry, and Andrew Lane. Distinctions between emotion and mood. Cognition \& Emotion, 19(6):847-878, 2005.

[20] Reda Belaiche, Yu Liu, Cyrille Migniot, Dominique Ginhac, and Fan Yang. Cost-effective cnns for real-time micro-expression recognition. Applied Sciences, 10(14):4959, 2020.

[21] Robert James R Blair. Fine cuts of empathy and the amygdala: dissociable deficits in psychopathy and autism. Quarterly journal of experimental psychology, 61(1):157-170, 2008.

[22] Paul Bloom. Empathy and its discontents. Trends in cognitive sciences, 21(1):24-31, 2017.

[23] Michael Boiger, Eva Ceulemans, Jozefien De Leersnyder, Yukiko Uchida, Vinai Norasakkunkit, and Batja Mesquita. Beyond essentialism: Cultural differences in emotions revisited. Emotion, 18(8):1142, 2018.

[24] Rafael H Bordini and Jomi F Hübner. A java-based interpreter for an extended version of agentspeak. University of Durham, Universidade Regional de Blumenau, 256, 2007. 
[25] Rafael H Bordini, Jomi Fred Hübner, and Michael Wooldridge. Programming multi-agent systems in AgentSpeak using Jason, volume 8. John Wiley \& Sons, 2007.

[26] Rafael H Bordini and Alvaro F Moreira. Proving BDI properties of agent-oriented programming languages: The asymmetry thesis principles in agentspeak. Annals of Mathematics and Artificial Intelligence, 42(1):197-226, 2004.

[27] Jeroen Borghuis, Wiebke Bleidorn, Klaas Sijtsma, Susan Branje, Wim HJ Meeus, and Jaap JA Denissen. Longitudinal associations between trait neuroticism and negative daily experiences in adolescence. Journal of Personality and Social Psychology, Ahead of print (2019), 2019 .

[28] Vicent Botti, Andrea Omicini, Stefano Mariani, and Vicente Julian. Multi-agent systems. MDPI-Multidisciplinary Digital Publishing Institute, 2019.

[29] Víctor Botti-Cebriá, Elena del Val, and Ana García-Fornes. Automatic detection of sensitive information in educative social networks. In Conference on Complex, Intelligent, and Software Intensive Systems, pages 184-194. Springer, 2020.

[30] Hana Boukricha and Ipke Wachsmuth. Modeling empathy for a virtual human: how, when and to what extent? In The 10th International Conference on Autonomous Agents and Multiagent Systems-Volume 3, pages 1135-1136, 2011.

[31] Hana Boukricha, Ipke Wachsmuth, Maria Nella Carminati, and Pia Knoeferle. A computational model of empathy: Empirical evaluation. In 2013 Humaine Association Conference on Affective Computing and Intelligent Interaction, pages 1-6. IEEE, 2013.

[32] Hana Boukricha, Ipke Wachsmuth, Maria Nella Carminati, and Pia Knoeferle. Empathy and its modulation in a virtual human. In Annual Conf. on Artificial Intelligence, pages 25-36. Springer, 2013. 
[33] Muriel Bouyer, Sophie Bagdassarian, Sveti Chaabanne, and Etienne Mullet. Personality correlates of risk perception. Risk analysis, 21(3):457-466, 2001.

[34] Paul H. Bucci, X Laura Cang, Hailey Mah, Laura Rodgers, and Karon E. MacLean. Real emotions don't stand still: Toward ecologically viable representation of affective interaction. In 8th Int. Conf. on Affective Computing and Intelligent Interaction (ACII), pages 1-7. IEEE, 2019.

[35] Sathya Bursic, Giuseppe Boccignone, Alfio Ferrara, Alessandro D'Amelio, and Raffaella Lanzarotti. Improving the accuracy of automatic facial expression recognition in speaking subjects with deep learning. Applied Sciences, 10(11):4002, 2020.

[36] Davide Buttarazzi, Giuseppe Pandolfo, and Giovanni C Porzio. A boxplot for circular data. Biometrics, 74(4):1492-1501, 2018.

[37] Linqin Cai, Binbin Liu, Jimin Yu, and Jianrong Zhang. Human behaviors modeling in multi-agent virtual environment. Multimedia Tools and Applications, 76(4):5851-5871, 2017.

[38] Sergio Castellanos, Luis-Felipe Rodríguez, Luis A Castro, and Joaquín Pérez. Cognitive modulation of appraisal variables in the emotion process of autonomous agents. In Cognitive Informatics 85 Cognitive Computing $\left(\right.$ ICCI $\left.^{*} C C\right)$, pages 102-108. IEEE, 2017.

[39] Sergio Castellanos, Luis-Felipe Rodríguez, and J Octavio GutierrezGarcia. A mechanism for biasing the appraisal process in affective agents. Cognitive Systems Research, 58:351-365, 2019.

[40] Ana Paula Chaves and Marco Aurelio Gerosa. How should my chatbot interact? A survey on social characteristics in human-chatbot interaction design. International Journal of Human-Computer Interaction, 37(8):729-758, 2021. 
[41] Marcello Cirillo, Lars Karlsson, and Alessandro Saffiotti. Human-aware task planning: an application to mobile robots. ACM Transactions on Intelligent Systems and Technology (TIST), 1(2):1-26, 2010.

[42] Caitlyn Clabaugh and Maja Matarić. Robots for the people, by the people: Personalizing human-machine interaction. Science robotics, 3(21):eaat7451, 2018.

[43] Malissa A Clark, Melissa M Robertson, and Stephen Young. "I feel your pain": A critical review of organizational research on empathy. Journal of Organizational Behavior, 40(2):166-192, 2019.

[44] Giovanna Colombetti. The embodied and situated nature of moods. Philosophia, 45(4):1437-1451, 2017.

[45] Amy Coplan and Peter Goldie. Empathy: Philosophical and psychological perspectives. Oxford University Press, 2011.

[46] Daniel T Cordaro, Rui Sun, Dacher Keltner, Shanmukh Kamble, Niranjan Huddar, and Galen McNeil. Universals and cultural variations in 22 emotional expressions across five cultures. Emotion, 18(1):75, 2018.

[47] Natalia Criado, Estefania Argente, Pablo Noriega, and Vicente Botti. Human-inspired model for norm compliance decision making. Information Sciences, 245:218-239, 2013.

[48] Ana Carla Crispim, Roberto Moraes Cruz, Cassandra Melo Oliveira, and Aline Battisti Archer. O afeto sob a perspectiva do circumplexo: evidências de validade de construto. Avaliação Psicológica, 16(2):145$152,2017$.

[49] Benjamin MP Cuff, Sarah J Brown, Laura Taylor, and Douglas J Howat. Empathy: A review of the concept. Emotion Review, 8(2):144-153, 2016.

[50] Mark H Davis. Empathy: A social psychological approach. Routledge, 2018. 
[51] Mark H Davis, Carol Luce, and Stephen J Kraus. The heritability of characteristics associated with dispositional empathy. Journal of personality, 62(3):369-391, 1994.

[52] Frederique De Vignemont and Tania Singer. The empathic brain: how, when and why? Trends in cognitive sciences, 10(10):435-441, 2006.

[53] Frans BM De Waal. The 'russian doll'model of empathy and imitation. On being moved: From mirror neurons to empathy, pages 35-48, 2007.

[54] Jean Decety and Philip L Jackson. The functional architecture of human empathy. Behavioral and cognitive neuroscience reviews, 3(2):71-100, 2004 .

[55] Jean Decety and Claus Lamm. Human empathy through the lens of social neuroscience. The scientific World journal, 6:1146-1163, 2006.

[56] Douglas Derryberry and Marjorie A Reed. Temperament and attention: Orienting toward and away from positive and negative signals. Journal of Personality and Social Psychology, 66(6):1128-1139, 1994.

[57] Sandrine Detandt, Christophe Leys, and Ariane Bazan. A french translation of the pleasure arousal dominance (PAD) semantic differential scale for the measure of affect and drive. Psychologica Belgica, 57(1):17, 2017.

[58] Cambridge Dictionary. Cambridge English-Spanish Dictionary. https://dictionary.cambridge.org, 2019 (accessed March 10, 2019).

[59] Collins Dictionary. Collins English-Spanish Dictionary. https://www.collinsdictionary.com, 2019 (accessed March 10, 2019).

[60] Oxford Dictionary. Oxford English-Spanish Dictionary. https://es.oxforddictionaries.com, 2019 (accessed March 10, 2019).

[61] Wordreference Dictionary. Wordreference English-Spanish Dictionary. https://www.wordreference.com, 2019 (accessed March 10, 2019). 
[62] Ulf Dimberg and Monika Thunberg. Empathy, emotional contagion, and rapid facial reactions to angry and happy facial expressions. PsyCh Journal, 1(2):118-127, 2012.

[63] Jiaxuan Du, Jiali Huang, Yuanyuan An, and Wei Xu. The relationship between stress and negative emotion: The mediating role of rumination. Clin Res, 4(1):1-5, 2018.

[64] Panteleimon Ekkekakis. Affect, mood, and emotion. In Measurement in Sport and Exercise Psychology, pages 321-1332. Human Kinetics, 2012.

[65] Paul Ekman. An argument for basic emotions. Cognition 6 Emotion, 6(3-4):169-200, 1992.

[66] Paul Ed Ekman and Richard J Davidson. The nature of emotion: Fundamental questions. Oxford University Press, 1994.

[67] Magy Seif El-Nasr, John Yen, and Thomas R Ioerger. Flame-fuzzy logic adaptive model of emotions. Autonomous Agents and Multi-agent systems, 3(3):219-257, 2000.

[68] Hillary Anger Elfenbein. 7 emotion in organizations: a review and theoretical integration. Academy of management annals, 1(1):315-386, 2007.

[69] Nacer Farajzadeh and Mahdi Hashemzadeh. Exemplar-based facial expression recognition. Information Sciences, 460-461:318 - 330, 2018.

[70] PF Ferrari, M Gerbella, G Coudé, and S Rozzi. Two different mirror neuron networks: the sensorimotor (hand) and limbic (face) pathways. Neuroscience, 358:300-315, 2017.

[71] Joseph P Forgas. Mood effects on cognition: Affective influences on the content and process of information processing and behavior. Emotions and affect in human factors and human-computer interaction, pages 89-122, 2017. 
[72] Nico H Frijda, Peter Kuipers, and Elisabeth Ter Schure. Relations among emotion, appraisal, and emotional action readiness. Journal of Personality and Social Psychology, 57(2):212, 1989.

[73] Chris D Frith and Uta Frith. The neural basis of mentalizing. Neuron, 50(4):531-534, 2006.

[74] José Manuel Fuentes, Joaquin Taverner, Jaime Andres Rincon, and Vicente Botti. Towards a classifier to recognize emotions using voice to improve recommendations. In International Conference on Practical Applications of Agents and Multi-Agent Systems, pages 218-225. Springer, 2020.

[75] Desire Furnes, Hege Berg, Rachel LC Mitchell, and Silke Paulmann. Exploring the effects of personality traits on the perception of emotions from prosody. Frontiers in Psychology, 10:184, 2019.

[76] Helen L Gallagher and Christopher D Frith. Functional imaging of 'theory of mind'. Trends in cognitive sciences, 7(2):77-83, 2003.

[77] Shaun Gallagher. The new hybrids: Continuing debates on social perception. Consciousness and cognition, 36:452-465, 2015.

[78] Joanna Ganczarek, Thomas Hünefeldt, and Marta Olivetti Belardinelli. From "einfühlung" to empathy: exploring the relationship between aesthetic and interpersonal experience. Cognitive processing, 19(2):141$145,2018$.

[79] Francisco J. García-Peñalvo and Nicholas Alfred Kearney. Networked youth research for empowerment in digital society: The wyred project. In Proceedings of the Fourth International Conference on Technological Ecosystems for Enhancing Multiculturality, page 3-9. Association for Computing Machinery, 2016.

[80] Bertram Gawronski, Paul Conway, Joel Armstrong, Rebecca Friesdorf, and Mandy Hütter. Effects of incidental emotions on moral dilemma judgments: An analysis using the cni model. Emotion, 18(7):989, 2018. 
[81] Patrick Gebhard. ALMA: a layered model of affect. In Proc.gs of the 4th Int. Joint Conf. on Autonomous Agents and Multiagent Systems, pages 29-36. ACM, 2005.

[82] Patrick Gebhard and Kerstin H Kipp. Are computer-generated emotions and moods plausible to humans? In Int. Workshop on Intelligent Virtual Agents, pages 343-356. Springer, 2006.

[83] Michael Gelfond and Vladimir Lifschitz. Action languages. Computer and Information Science, 3(16), 1998.

[84] Gonzalo Blázquez Gil, Antonio Berlanga de Jesús, and José M Molina Lopéz. Combining machine learning techniques and natural language processing to infer emotions using spanish twitter corpus. In International Conference on Practical Applications of Agents and Multi-Agent Systems, pages 149-157. Springer, 2013.

[85] Joao Gluz and Patricia A Jaques. A probabilistic formalization of the appraisal for the occ event-based emotions. Journal of Artificial Intelligence Research, 58:627-664, 2017.

[86] Alvin Goldman. Two routes to empathy. In Empathy: Philosophical and psychological perspectives, pages 31-44. Oxford University Press Oxford, 2011.

[87] José Gómez-Galán, José Ángel Martínez-López, Cristina Lázaro-Pérez, and José Luis Sarasola Sánchez-Serrano. Social networks consumption and addiction in college students during the covid-19 pandemic: Educational approach to responsible use. Sustainability, 12(18):7737, 2020.

[88] Jonathan Gratch and Stacy Marsella. A domain-independent framework for modeling emotion. Cognitive Systems Research, 5(4):269-306, 2004.

[89] Beate Grawemeyer, Hilary Johnson, Mark Brosnan, Emma Ashwin, and Laura Benton. Developing an embodied pedagogical agent with and for young people with autism spectrum disorder. In International 
Conference on Intelligent Tutoring Systems, pages 262-267. Springer, 2012.

[90] Elizabeth K Gray, David Watson, R Payne, and C Cooper. Emotion, mood, and temperament: Similarities, differences, and a synthesis. In Emotions at work: Theory, research and applications for management, pages 21-43. John Wiley \& Sons Chichester, UK, 2001.

[91] James J Gross. Emotion regulation: Conceptual and empirical foundations. The Guilford Press, 2014.

[92] Ayesha Hakim, Stephen Marsland, and Hans W Guesgen. Statistical modelling of complex emotions using mixture of von mises distributions. In 2013 Humaine Association Conf. on Affective Computing and Intelligent Interaction, pages 517-522. IEEE, 2013.

[93] Eddie Harmon-Jones, Cindy Harmon-Jones, and Elizabeth Summerell. On the importance of both dimensional and discrete models of emotion. Behavioral sciences, 7(4):66, 2017.

[94] Grit Hein and Tania Singer. I feel how you feel but not always: the empathic brain and its modulation. Current opinion in neurobiology, 18(2):153-158, 2008.

[95] Cecilia Heyes. Where do mirror neurons come from? Neuroscience 86 Biobehavioral Reviews, 34(4):575-583, 2010.

[96] Cecilia Heyes. Empathy is not in our genes. Neuroscience \& Biobehavioral Reviews, 95:499-507, 2018.

[97] Katie Hoemann, Fei Xu, and Lisa Feldman Barrett. Emotion words, emotion concepts, and emotional development in children: A constructionist hypothesis. Developmental psychology, 55(9):1830, 2019.

[98] Martin L Hoffman. Interaction of affect and cognition in empathy. In Emotions, cognition, and behavior, pages 103-131. Cambridge University Press, 1984. 
[99] Martin L Hoffman. Empathy and moral development: Implications for caring and justice. Cambridge University Press, 2001.

[100] Martin L Hoffman. Empathy and prosocial behavior. Handbook of emotions, 3:440-455, 2008.

[101] Ying-yi Hong, Michael W Morris, Chi-yue Chiu, and Veronica BenetMartinez. Multicultural minds: A dynamic constructivist approach to culture and cognition. American psychologist, 55(7):709, 2000.

[102] Joshua T Horwood and Aubrey B Poore. Gauss von mises distribution for improved uncertainty realism in space situational awareness. SIAM/ASA Journal on uncertainty Quantification, 2(1):276-304, 2014.

[103] M Shamim Hossain and Ghulam Muhammad. Emotion recognition using secure edge and cloud computing. Information Sciences, 504:589601, 2019.

[104] Marco Iacoboni. Imitation, empathy, and mirror neurons. Annual review of psychology, 60:653-670, 2009.

[105] Infopedia Dictionary. Infopedia English-Portuguese Dictionary. https://www.infopedia.pt, 2021 (accessed September 17, 2021).

[106] Leonid Ivonin, Huang-Ming Chang, Wei Chen, Matthias Rauterberg, et al. A new representation of emotion in affective computing. In Proc. of Int. Conf. on affective computing and intelligent interaction, pages 337-343, 2012.

[107] Joshua Conrad Jackson, Joseph Watts, Teague R. Henry, JohannMattis List, Robert Forkel, Peter J. Mucha, Simon J. Greenhill, Russell D. Gray, and Kristen A Lindquist. Emotion semantics show both cultural variation and universal structure. Science, 366(6472):1517$1522,2019$.

[108] Shikha Jain and Krishna Asawa. EMIA: emotion model for intelligent agent. Journal of Intelligent Systems, 24(4):449-465, 2015. 
[109] S Rao Jammalamadaka and Ambar Sengupta. Topics in circular statistics, volume 5. World Scientific, 2001.

[110] Lina Jasmontaite and Paul De Hert. The eu, children under 13 years, and parental consent: a human rights analysis of a new, age-based bright-line for the protection of children on the internet. International Data Privacy Law, 5(1):20-33, 2015.

[111] Felix Jimenez, Tomohiro Yoshikawa, Takeshi Furuhashi, and Masayoshi Kanoh. An emotional expression model for educational-support robots. Journal of Artificial Intelligence and Soft Computing Research, 5, 2015.

[112] George H John and Pat Langley. Estimating continuous distributions in bayesian classifiers. In Proceedings of the 11th Conference on Uncertainty in Artificial Intelligence, pages 338-345. Morgan Kaufmann Publishers Inc., 1995.

[113] Timotheus Kampik, Juan Carlos Nieves, and Helena Lindgren. Empathic autonomous agents. In International Workshop on Engineering Multi-Agent Systems, pages 181-201. Springer, 2018.

[114] Hasan Karal, Mehmet Kokoc, and Ozlem Cakir. Impact of the educational use of facebook group on the high school students' proper usage of language. Education and Information Technologies, 22(2):677-695, 2017.

[115] Fakhreddine Karray, Milad Alemzadeh, Jamil Abou Saleh, and Mo Nours Arab. Human-computer interaction: Overview on state of the art. International journal on smart sensing and intelligent systems, $1(1), 2017$.

[116] Charalampos Karyotis, Faiyaz Doctor, Rahat Iqbal, Anne James, and Victor Chang. A fuzzy computational model of emotion for cloud based sentiment analysis. Information Sciences, 433:448-463, 2018. 
[117] Zerrin Kasap, Maher Ben Moussa, Parag Chaudhuri, and Nadia Magnenat-Thalmann. Making them remember - emotional virtual characters with memory. IEEE Computer Graphics and Applications, 29(2):20-29, 2009.

[118] Christina Katsimerou, Judith A Redi, and Ingrid Heynderickx. A computational model for mood recognition. In International Conference on User Modeling, Adaptation, and Personalization, pages 122-133. Springer, 2014.

[119] Antti Kauppinen. Empathy, emotion regulation, and moral judgment. Oxford University Press, 2014.

[120] Anna Kåver. KBT i utveckling: en introduktion till kognitiv beteendeterapi. Natur och kultur, 2006.

[121] Abe Kazemzadeh. Using interval type-2 fuzzy logic to translate emotion words from Spanish to English. In Int. Conf. on Fuzzy Systems, pages 1-8. IEEE, 2010.

[122] Abe Kazemzadeh, Sungbok Lee, and Shrikanth Narayanan. Fuzzy logic models for the meaning of emotion words. IEEE Computational Intelligence, 8(2):34-49, 2013.

[123] Abe Kazemzadeh, Sungbok Lee, and Shrikanth S Narayanan. An interval type-2 fuzzy logic system to translate between emotion-related vocabularies. In Ninth Annual Conference of the International Speech Communication Association, 2008.

[124] Theodore D Kemper. Elementary Forms of Social Relations: Status, power and reference groups. Routledge, 2016.

[125] Olga M Klimecki, Matthieu Vétois, and David Sander. The impact of empathy and perspective-taking instructions on proponents and opponents of immigration. Humanities and Social Sciences Communications, $7(1): 1-12,2020$. 
[126] George Klir and Bo Yuan. Fuzzy sets and fuzzy logic, volume 4. Prentice Hall New Jersey, 1995.

[127] Igor Knez and Staffan Hygge. The circumplex structure of affect: A swedish version. Scandinavian Journal of Psychology, 42(5):389-398, 2001.

[128] Monica La Mura and Patrizia Lamberti. Human-machine interaction personalization: a review on gender and emotion recognition through speech analysis. In 2020 IEEE International Workshop on Metrology for Industry 4.0 \& Io T, pages 319-323. IEEE, 2020.

[129] Claus Lamm, C Daniel Batson, and Jean Decety. The neural substrate of human empathy: effects of perspective-taking and cognitive appraisal. Journal of cognitive neuroscience, 19(1):42-58, 2007.

[130] Claus Lamm, Markus Rütgen, and Isabella C Wagner. Imaging empathy and prosocial emotions. Neuroscience letters, 693:49-53, 2019.

[131] Agnieszka Landowska. Towards new mappings between emotion representation models. Applied Sciences, 8(2):274, 2018.

[132] Randy J Larsen and Edward Diener. Promises and problems with the circumplex model of emotion. In M. S. Clark (Ed.), Emotion (pp. 25-59), 1992.

[133] Randy J Larsen and Timothy Ketelaar. Personality and susceptibility to positive and negative emotional states. Journal of Personality and Social Psychology, 61(1):132, 1991.

[134] Jennifer C Lay, Denis Gerstorf, Stacey B Scott, Theresa Pauly, and Christiane A Hoppmann. Neuroticism and extraversion magnify discrepancies between retrospective and concurrent affect reports. Journal of Personality, 85(6):817-829, 2017.

[135] Richard S Lazarus. Emotion and adaptation. Oxford University Press, 1991. 
[136] Nicole Lazzeri, Daniele Mazzei, Lorenzo Cominelli, Antonio Cisternino, and Danilo Emilio De Rossi. Designing the mind of a social robot. Applied Sciences, 8(2):302, 2018.

[137] Colin Wayne Leach, Russell Spears, and Antony SR Manstead. Parsing (malicious) pleasures: Schadenfreude and gloating at others' adversity. Frontiers in Psychology, 6, 2015.

[138] Iolanda Leite, Ginevra Castellano, André Pereira, Carlos Martinho, and Ana Paiva. Empathic robots for long-term interaction. International Journal of Social Robotics, 6(3):329-341, 2014.

[139] Iolanda Leite, Carlos Martinho, and Ana Paiva. Social robots for longterm interaction: a survey. International Journal of Social Robotics, 5(2):291-308, 2013.

[140] Heather C Lench and Zari Koebel Carpenter. What do emotions do for us? In The Function of Emotions, pages 1-7. Springer, 2018.

[141] Nangyeon Lim. Cultural differences in emotion: differences in emotional arousal level between the east and the west. Integrative Medicine Research, 5(2):105 - 109, 2016.

[142] Patricia L Lockwood and Miriam C Klein-Flügge. Computational modelling of social cognition and behaviour - a reinforcement learning primer. Social cognitive and affective neuroscience, 2020.

[143] Anibal Antonio Lopez, Paulo Renato Lourenço, Isabel Dimas, and Cludia Figueiredo. Pjawsn-escala portuguesa do bem-estar afectivo no trabalho: Contributos para a sua validação. A emoção nas organizações, pages 155-178, 2012.

[144] Juan M Machimbarrena, Esther Calvete, Liria Fernández-González, Aitor Álvarez-Bardón, Lourdes Álvarez-Fernández, and Joaquin González-Cabrera. Internet risks: An overview of victimization in cyberbullying, cyber dating abuse, sexting, online grooming and problem- 
atic internet use. International journal of environmental research and public health, 15(11):2471, 2018.

[145] Alexandra Main and Carmen Kho. A relational framework for integrating the study of empathy in children and adults. Emotion Review, 12(4):280-290, 2020.

[146] Javier Marín-Morales, Juan Higuera-Trujillo, Alberto Greco, Jaime Guixeres, Carmen Llinares, Enzo Scilingo, Mariano Alcañiz, and Gaetano Valenza. Affective computing in virtual reality: emotion recognition from brain and heartbeat dynamics using wearable sensors. Scientific reports, 8(1), 2018.

[147] Stacy Marsella, Jonathan Gratch, Paolo Petta, et al. Computational models of emotion. A Blueprint for Affective Computing, 11(1):21-46, 2010 .

[148] Stacy C Marsella and Jonathan Gratch. EMA: A process model of appraisal dynamics. Cognitive Systems Research, 10(1):70-90, 2009.

[149] Abigail A Marsh. The neuroscience of empathy. Current opinion in behavioral sciences, 19:110-115, 2018.

[150] Joseph W Matiko, Stephen P Beeby, and John Tudor. Fuzzy logic based emotion classification. In IEEE Int. Conf. on Acoustics, Speech and Signal Processing (ICASSP), pages 4389-4393, 2014.

[151] David Matsumoto and Linda Juang. Culture and Psychology. Nelson Education, 2016.

[152] Robert R McCrae and Oliver P John. An introduction to the five-factor model and its applications. Journal of Personality, 60(2):175-215, 1992.

[153] Albert Mehrabian. Analysis of the big-five personality factors in terms of the PAD temperament model. Australian journal of Psychology, 48(2):86-92, 1996. 
[154] Albert Mehrabian. Pleasure-arousal-dominance: A general framework for describing and measuring individual differences in temperament. Current Psychology, 14(4):261-292, 1996.

[155] Ben Meuleman and Klaus R Scherer. Nonlinear appraisal modeling: An application of machine learning to the study of emotion production. IEEE Trans. on Affective Computing, 4(4):398-411, 2013.

[156] Marina Micheli. Social networking sites and low-income teenagers: between opportunity and inequality. Information, Communication 85 Society, 19(5):565-581, 2016.

[157] Sylvia A Morelli, Matthew D Lieberman, and Jamil Zaki. The emerging study of positive empathy. Social and Personality Psychology Compass, 9(2):57-68, 2015.

[158] Juan E Moreno, Mauricio A Sanchez, Olivia Mendoza, Antonio Rodríguez-Díaz, Oscar Castillo, Patricia Melin, and Juan R Castro. Design of an interval type-2 fuzzy model with justifiable uncertainty. Information Sciences, 513:206-221, 2020.

[159] Mohammad Obaid, Ruth Aylett, Wolmet Barendregt, Christina Basedow, Lee J Corrigan, Lynne Hall, Aidan Jones, Arvid Kappas, Dennis Küster, Ana Paiva, et al. Endowing a robotic tutor with empathic qualities: Design and pilot evaluation. International Journal of Humanoid Robotics, 15(06):1850025, 2018.

[160] Patrick D O'Brien and Richard C Nicol. Fipa-towards a standard for software agents. BT Technology Journal, 16(3):51-59, 1998.

[161] Magalie Ochs, David Sadek, and Catherine Pelachaud. A formal model of emotions for an empathic rational dialog agent. Autonomous Agents and Multi-Agent Systems, 24(3):410-440, 2012.

[162] Kevin N Ochsner and Elizabeth Phelps. Emerging perspectives on emotion-cognition interactions. Trends in Cognitive Sciences, 11(8):317-318, 2007. 
[163] Andrew Ortony, Gerald L Clore, and Allan Collins. The cognitive structure of emotions. Cambridge University Press, 1990.

[164] Mark D Packard and Thomas A Burnham. Do we understand each other? toward a simulated empathy theory for entrepreneurship. Journal of Business Venturing, 36(1):106076, 2021.

[165] Ana Paiva, Iolanda Leite, Hana Boukricha, and Ipke Wachsmuth. Empathy in virtual agents and robots: a survey. ACM Trans. on Interactive Intelligent Systems (TiiS), 7(3):11, 2017.

[166] Stefan Palan and Christian Schitter. Prolific. ac - a subject pool for online experiments. Journal of Behavioral and Experimental Finance, $17: 22-27,2018$.

[167] Brian Parkinson, Peter Totterdell, Rob B Briner, and Shirley Reynolds. Changing moods: The psychology of mood and mood regulation. Longman London, 1996.

[168] Bert Peeters. Semantic primes and universal grammar: Empirical evidence from the Romance languages, volume 81. John Benjamins Publishing, 2006.

[169] Arthur Pewsey, Markus Neuhäuser, and Graeme D Ruxton. Circular statistics in R. Oxford University Press, 2013.

[170] Rosalind Wright Picard. Affective Computing. The MIT Press, 1997.

[171] Gordon D Plotkin. A structural approach to operational semantics. Aarhus university, 1981.

[172] Robert Plutchik. The nature of emotions. American Scientist, 89:344350, 2001.

[173] Soujanya Poria, Erik Cambria, Rajiv Bajpai, and Amir Hussain. A review of affective computing: From unimodal analysis to multimodal fusion. Information Fusion, 37:98-125, 2017. 
[174] Jonathan Posner, James A Russell, and Bradley S Peterson. The circumplex model of affect: An integrative approach to affective neuroscience, cognitive development, and psychopathology. Development and Psychopathology, 17(3):715-734, 2005.

[175] Stephanie D Preston. A perception-action model for empathy. Empathy in mental illness, 1:428-447, 2007.

[176] Stephanie D Preston and Frans BM De Waal. Empathy: Its ultimate and proximate bases. Behavioral and brain sciences, 25(1):1-20, 2002.

[177] Priberam Dictionary. Priberam English-Swedish Dictionary. https://dicionario.priberam.org, 2021 (accessed September 17, 2021).

[178] Brian A Primack, Sabrina A Karim, Ariel Shensa, Nicholas Bowman, Jennifer Knight, and Jaime E Sidani. Positive and negative experiences on social media and perceived social isolation. American Journal of Health Promotion, 33(6):859-868, 2019.

[179] Anand S Rao. Agentspeak (1): Bdi agents speak out in a logical computable language. In European workshop on modelling autonomous agents in a multi-agent world, pages 42-55. Springer, 1996.

[180] Anand S Rao, Michael P Georgeff, et al. BDI agents: from theory to practice. In 1st Int. Conf. on Multiagent Systems, volume 95, pages 312-319, 1995.

[181] Zeeshan Rasool, Naoki Masuyama, Md Nazrul Islam, and Chu Kiong Loo. Empathic interaction using the computational emotion model. In 2015 IEEE Symposium Series on Computational Intelligence, pages 109-116. IEEE, 2015.

[182] Rainer Reisenzein, Eva Hudlicka, Mehdi Dastani, Jonathan Gratch, Koen Hindriks, Emiliano Lorini, and John-Jules Ch Meyer. Computational modeling of emotion: Toward improving the inter- and intradisciplinary exchange. IEEE Trans. on Affective Computing, 4(3):246-266, 2013. 
[183] Ranier Reisenzein. Pleasure-arousal theory and the intensity of emotions. Journal of Personality and Social Psychology, 67(3):525, 1994.

[184] Helen Riess. The science of empathy. Journal of patient experience, $4(2): 74-77,2017$.

[185] Jaime A Rincon, Angelo Costa, Paulo Novais, Vicente Julián, and Carlos Carrascosa. A dynamic emotional model for agent societies. In Int. Conf. on Practical Applications of Agents and Multi-Agent Systems, pages 169-182. Springer, 2016.

[186] Jaime A Rincon, Angelo Costa, Paulo Novais, Vicente Julian, and Carlos Carrascosa. Using emotions in intelligent virtual environments: the ejacalive framework. Wireless Communications and Mobile Computing, 2017, 2017.

[187] Giacomo Rizzolatti, Luciano Fadiga, Vittorio Gallese, and Leonardo Fogassi. Premotor cortex and the recognition of motor actions. Cognitive brain research, 3(2):131-141, 1996.

[188] Sérgio Hortas Rodrigues, Samuel Mascarenhas, João Dias, and Ana Paiva. A process model of empathy for virtual agents. Interacting with Computers, 27(4):371-391, 2015.

[189] Luis-Felipe Rodríguez, J Octavio Gutierrez-Garcia, and Félix Ramos. Modeling the interaction of emotion and cognition in autonomous agents. Biologically Inspired Cognitive Architectures, 17:57-70, 2016.

[190] Ira J Roseman and Craig A Smith. Appraisal theory: Overview, assumptions, varieties, controversies. In Appraisal Processes in Emotion: Theory, Methods, Research, pages 3-19. Oxford University Press, 2001.

[191] Ramon Ruiz-Dolz, Stella Heras, José Alemany, and Ana García-Fornes. Towards an argumentation system for assisting users with privacy management in online social networks. In CMNA@PERSUASIVE, pages $17-28,2019$. 
[192] James A Russell. Evidence of convergent validity on the dimensions of affect. Journal of personality and social psychology, 36(10):1152, 1978.

[193] James A Russell. A circumplex model of affect. Journal of Personality and Social Psychology, 39(6):1161-1178, 1980.

[194] James A Russell. Core affect and the psychological construction of emotion. Psychological Review, 110(1):145, 2003.

[195] James A Russell and Lisa Feldman Barrett. Core affect, prototypical emotional episodes, and other things called emotion: dissecting the elephant. Journal of Personality and Social Psychology, 76(5):805, 1999.

[196] James A Russell, Maria Lewicka, and Toomas Niit. A cross-cultural study of a circumplex model of affect. Journal of Personality and Social Psychology, 57(5):848-856, 1989.

[197] Cheryl L Rusting and Randy J Larsen. Extraversion, neuroticism, and susceptibility to positive and negative affect: A test of two theoretical models. Personality and individual differences, 22(5):607-612, 1997.

[198] Jelle Saldien, Kristof Goris, Bram Vanderborght, Johan Vanderfaeillie, and Dirk Lefeber. Expressing emotions with the social robot probo. Int. Journal of Social Robotics, 2(4):377-389, 2010.

[199] Miguel A Salichs and Maria Malfaz. A new approach to modeling emotions and their use on a decision-making system for artificial agents. IEEE Trans. on Affective Computing, 3(1):56-68, 2012.

[200] Klaus R Scherer. Studying the emotion-antecedent appraisal process: An expert system approach. Cognition 86 Emotion, 7(3-4):325-355, 1993.

[201] Klaus R Scherer, Angela Schorr, and Tom Johnstone. Appraisal processes in emotion: Theory, Methods, Research. Oxford University Press, 2001. 
[202] Harold Schlosberg. The description of facial expressions in terms of two dimensions. Journal of experimental psychology, 44(4):229, 1952.

[203] Marc Schröder, Paolo Baggia, Felix Burkhardt, Catherine Pelachaud, Christian Peter, and Enrico Zovato. Emotionml-an upcoming standard for representing emotions and related states. In Int. Conf. on Affective Computing and Intelligent Interaction, pages 316-325. Springer, 2011.

[204] Frank Schweitzer, Tamas Krivachy, and David Garcia. An agent-based model of opinion polarization driven by emotions. Complexity, 2020, 2020 .

[205] Elizabeth A Segal. Social empathy: A new paradigm to address poverty. Journal of Poverty, 11(3):65-81, 2007.

[206] Suzanne C Segerstrom and Gregory T Smith. Personality and coping: Individual differences in responses to emotion. Annual review of psychology, 70:651-671, 2019.

[207] Sofia Serholt and Wolmet Barendregt. Robots tutoring children: Longitudinal evaluation of social engagement in child-robot interaction. In Proceedings of the 9th nordic conference on human-computer interaction, pages 1-10, 2016.

[208] Nigel Shadbolt, Paul R Smart, JR Wilson, and S Sharples. Knowledge elicitation. Evaluation of human work, pages 163-200, 2015.

[209] Ali Sharifara, Mohd Shafry Mohd Rahim, and Yasaman Anisi. A general review of human face detection including a study of neural networks and haar feature-based cascade classifier in face detection. In 2014 International Symposium on Biometrics and Security Technologies (ISBAST), pages 73-78. IEEE, 2014.

[210] Cristiana S Silva, Glívia AR Barbosa, Ismael S Silva, Tatiane S Silva, Fernando Mourão, and Flávio Coutinho. Privacy for children and teenagers on social networks from a usability perspective: a case study 
on facebook. In Proceedings of the 2017 ACM on Web Science Conference, pages 63-71, 2017.

[211] Lennart Sjöberg, Elisabeth Engelberg, et al. Emotionell intelligens-teori och empiri i ett psykologiskt perspektiv. Emotional intelligence-theory and empirical research in a psychological perspective, 2003.

[212] Julia Stietz, Emanuel Jauk, Sören Krach, and Philipp Kanske. Dissociating empathy from perspective-taking: Evidence from intra-and inter-individual differences research. Frontiers in psychiatry, 10:126, 2019.

[213] Karsten Stueber. Empathy. International Encyclopedia of Ethics, 2013.

[214] Synonymer Dictionary. Synonymer Swedish dictionary of synonyms. https://www.synonymer.se, 2021 (accessed September 17, 2021).

[215] Joaquín Taverner, Bexy Alfonso, Emilio Vivancos, and Vicent J. Botti. Using different agent personalities to obtain different agent behaviours. In Proc.gs of the Workshop on Affective Computing and Context Awareness in Ambient Intelligence (AfCAI 2018), 2018.

[216] Joaquin Taverner, Bexy Alfonso, Emilio Vivancos, and Vicente Botti. Modeling personality in the affective agent architecture GenIA3. In Int. Conf. on Agents and Artificial Intelligence (ICAART), pages 236-243, 2018.

[217] Joaquin Taverner, Bexy Alfonso, Emilio Vivancos, and Vicente J Botti. Integrating expectations into Jason for appraisal in emotion modeling. In IJCCI (ECTA), pages 231-238, 2016.

[218] Joaquin Taverner, Ramon Ruiz, Elena del Val, Carlos Diez, and Jose Alemany. Image analysis for privacy assessment in social networks. In International Symposium on Distributed Computing and Artificial Intelligence, pages 1-4. Springer, 2018. 
[219] Joaquín Taverner, Emilio Vivancos, and Vicente Botti. Towards a computational approach to emotion elicitation in affective agents. In International Conference on Agents and Artificial Intelligence, pages 275280, 2019.

[220] Joaquin Taverner, Emilio Vivancos, and Vicente Botti. A multidimensional culturally adapted representation of emotions for affective computational simulation and recognition. IEEE Transactions on Affective Computing, 2020.

[221] Joaquin Taverner, Emilio Vivancos, and Vicente Botti. A fuzzy appraisal model for affective agents adapted to cultural environments using the pleasure and arousal dimensions. Information Sciences, 546:7486, 2021.

[222] Auke Tellegen, David Watson, and Lee Anna Clark. On the dimensional and hierarchical structure of affect. Psychological science, 10(4):297303, 1999.

[223] Paul T Van Katwyk, Suzy Fox, Paul E Spector, and E Kevin Kelloway. Using the job-related affective well-being scale (jaws) to investigate affective responses to work stressors. Journal of occupational health psychology, 5(2):219, 2000.

[224] Renata Vieira, Álvaro F Moreira, Michael Wooldridge, and Rafael H Bordini. On the formal semantics of speech-act based communication in an agent-oriented programming language. Journal of Artificial Intelligence Research, 29:221-267, 2007.

[225] Douglas N Walton. Practical reasoning. University of California Press, 2020.

[226] Henry M Wellman. Theory of mind: The state of the art. European Journal of Developmental Psychology, 15(6):728-755, 2018.

[227] Anna Wierzbicka. Human emotions: universal or culture-specific? American Anthropologist, 88(3):584-594, 1986. 
[228] Travis J Wiltshire, Emilio JC Lobato, Daniel S McConnell, and Stephen M Fiore. Prospects for direct social perception: a multitheoretical integration to further the science of social cognition. Frontiers in Human Neuroscience, 8:1007, 2015.

[229] Lauren Wisp. History of the concept of empathy. Empathy and its development, pages 17-37, 1987.

[230] Lauren Wispé. The psychology of sympathy. Springer Science \& Business Media, 1991.

[231] Joshua Wondra and Phoebe Ellsworth. An appraisal theory of empathy and other vicarious emotional experiences. Psychological review, 122(3):411, 2015.

[232] Markus Wrbouschek and Thomas Slunecko. Moods in transition: Theorizing the affective-dynamic constitution of situatedness. New Ideas in Psychology, 62:100857, 2021.

[233] Özge Nilay Yalçın. Evaluating empathy in artificial agents. arXiv preprint arXiv:1908.05341, 2019.

[234] Özge Nilay Yalçın. Empathy framework for embodied conversational agents. Cognitive Systems Research, 59:123-132, 2020.

[235] Özge Nilay Yalçın and Steve DiPaola. A computational model of empathy for interactive agents. Biologically inspired cognitive architectures, $26: 20-25,2018$.

[236] Özge Nilay Yalçın and Steve DiPaola. Modeling empathy: building a link between affective and cognitive processes. Artificial Intelligence Review, pages 1-24, 2019.

[237] Michelle S M Yik, James A Russell, Chang-Kyu Ahn, José Miguel Fernández Dols, and Naoto Suzuki. Relating the five-factor model of personality to a circumplex model of affect: A five-language study. In The five-factor model of personality across cultures, pages 79-104. Springer, 2002. 
[238] Lotfi A Zadeh. Fuzzy sets. Information and control, 8(3):338-353, 1965.

[239] Lotfi Asker Zadeh. The concept of a linguistic variable and its application to approximate reasoning. In Learning systems and intelligent robots, pages 1-10. Springer, 1974.

[240] Tomasz Zaleskiewicz and Jakub Traczyk. Emotions and financial decision making. In Psychological perspectives on financial decision making, pages 107-133. Springer, 2020.

[241] Eduard Zell, Katja Zibrek, and Rachel McDonnell. Perception of virtual characters. In ACM Siggraph 2019 Courses, pages 1-17. ACM, 2019.

[242] Guozhen Zhao, Yan Ge, Biying Shen, Xingjie Wei, and Hao Wang. Emotion analysis for personality inference from EEG signals. IEEE Trans. on Affective Computing, 9(3):362-371, 2018.

[243] Ke Zhong, Tianwei Qiao, and Liqun Zhang. A study of emotional communication of emoticon based on russell's circumplex model of affect. In International Conference on Human-Computer Interaction, pages 577596. Springer, 2019. 